\title{
ON THE DISTRIBUTION OF THROUGHPUT OF TRANSFER LINES
}

\author{
A THESIS \\ SUBMITTED TO THE DEPARTMENT OF INDUSTRIAL \\ ENGINEERING \\ AND THE INSTITUTE OF ENGINEERING AND SCIENCES \\ OF BILKENT UNIVERSITY \\ IN PARTIAL FULFILLMENT OF THE REQUIREMENTS \\ FOR THE DEGREE OF \\ MASTER OF SCIENCE
}

\author{
By \\ Bahar Deler \\ June, 1998
}


. 45

8143203

1998 
I certify that I have read this thesis and that in my opinion it is fully adequate, in scope and in quality, as a thesis for the degree of Master of Science.

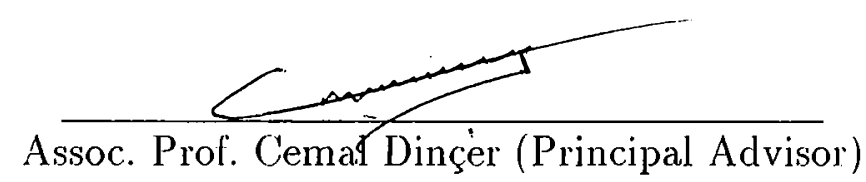

I certify that I have read this thesis and that in my opinion it is fully adequate, in scope and in quality, as a thesis for the degree of Master of Science.

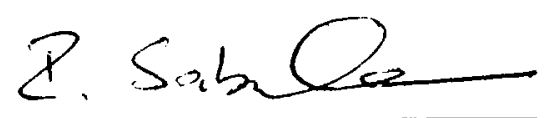

Assoc. Prof. İhsan Sabuncuog̃lu

I certify that I have read this thesis and that in my opinion it is fully adequate, in scope and in quality, as a thesis for the degree of Master of Science.

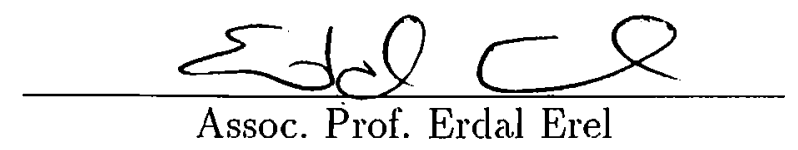

Approved for the Institute of Engineering and Sciences:

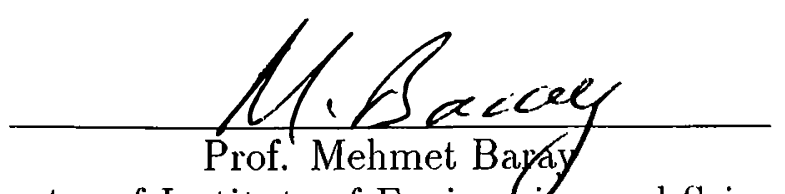

Director of Institute of Engineding and Sciences 


\title{
ABSTRACT \\ ON THE DISTRIBUTION OF THROUGHPUT OF TRANSFER LINES
}

\author{
Bahar Deler \\ M.S. in Industrial Engineering \\ Supervisor: Assoc. Prof. Cemal Dinçer \\ June, 1998
}

A transfer line corresponds to a manufacturing system consisting of a number of work stations in series integrated into one system by a common transfer mechanism and a control system. There is a vast literature on the transfer lines. However, little has been done on the transient analysis of these systems by making use of the higher order moments of their performance measures due to the difficulty in determining the evolution of the stochastic processes under consideration. This thesis examines the transient behavior of relatively short transfer lines and derives the distribution of the performance measures of interest. The proposed method based on the analytical derivation of the distribution of throughput is also applied to the systems with two-part types. An experiment is designed in order to compare the results of this study with the state-space representations and the simulation. They are also interpreted from the point of view of the line behavior and design issue. Furthermore, extensions are briefly discussed and directions for future research are suggested.

Key words: Distribution of Throughput, Transfer Lines, Stochastic Process Evolution, Transient Analysis, Steady-State Analysis. 


\title{
ÖZET
}

\section{SERI ÜRETIM HATLARINDA İ̧̧ ÇIKARMA YETENEG̈ININ DAĞILIMI ÜZERINE...}

\author{
Bahar Deler \\ Endüstri Mühendisliği Bölümü Yüksek Lisans \\ Tez Yöneticisi: Doç. Cemal Dinçer \\ Haziran, 1998
}

Seri üretim hatları parça akışını ve üretim aşamalarının etkileşimini en basit biçimde temsil etmektedir. Bu sistemlerle ilgili geniş bir literatür bulunmaktadır. Fakat, söz konusu rassal süreç evriminin belirlenmesindeki zorluk sebebiyle başarım ölçütlerinin yüksek sıra momentleri kullanılarak seri üretim hatlarının geçici durum çözümlemeleri üzerine eg̃ilinmemiştir. Bu çalışmada seri üretim hatlarının geçici durumu incelenmektedir. Ayrıca, önerilen yöntem üssel dağ̊lan aravarış ve işgörü sürelerine sahip çok parçalı sistemlere de uygulanmıştır. Bu çalışmada kullanılan yöntem, sonuçlarının durum uzayı betimleme ve benzetim sonuçları ile kıyaslanmasıyla doğrulanmıstır. Bunların yanısıra, sonuçlar hat davranışı ve tasarımı açısından da deg̃erlendirilmektedir. Ayrıca, yeni araştırma noktalarına da işaret edilmektedir.

Anahtar sözcükler: İ̧̧ Çıkarma Yeteneg̃inin Dağılımı, Seri Üretim Hatları, Rassal Süreç Evrimi, Geçici Durum Çözümlemesi, Kalıcı Durum Çözümlemesi. 
To my mother, to my father, and to Onur 


\section{ACKNOWLEDGEMENT}

I would like to express my gratitude to Assoc. Prof. Cemal Dinçer due to his supervision, suggestions, and understanding to bring this thesis to an end.

I am indebted to Assoc. Prof. İhsan Sabuncuoĝlu and Assoc. Prof. Erdal Erel for showing keen interest to the subject matter and accepting to read and review this thesis.

I would like to thank Muhittin Demir, Nebahat Dönmez, Lütfiye Durak, Bahar Kara, Alev Kaya, Gürhan Kök, Hakan Özaktaş, Eylem Tekin, and Ayten Türkcan for their moral support and help during the preparation of this thesis. 


\section{Contents}

1 Introduction 1

2 Serial Line Production System: A review 5

2.1 Steady-state behavior . . . . . . . . . . . . . 5

2.2 Transient behavior characterization ............ 13

2.2.1 Evolution of the work on the transient analysis of serial

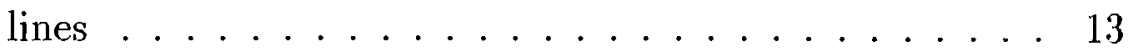

$\begin{array}{lll}2.2 .2 \text { A topology } & 15\end{array}$

$\begin{array}{lll}2.3 & \text { Summary } & 46\end{array}$

3 On the Distribution of Throughput 47

3.1 Introduction . . . . . . . . . . . . . . 47

3.1.1 The model assumptions and notation . . . . . . . . 47

$\begin{array}{lll}3.1 .2 & \text { Organization } & 48\end{array}$

3.2 The model development . . . . . . . . . . . . . . 50

$\begin{array}{ll}3.2 .1 & \text { Modeling }\end{array}$

vii 
$3.2 .2 \quad$ Approach . . . . . . . . . . . . . . 53

3.3 Numerical Results . . . . . . . . . . . . . . . . 57

3.3.1 Computer Codes . . . . . . . . . . . . . 57

3.3 .2 Validation . . . . . . . . . . . . 59

3.4 Summary 63

4 Extension of the Method: Two-Part Types 65

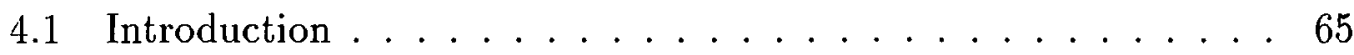

4.1.1 The model assumptions and notation . . . . . . 66

$\begin{array}{lll}\text { 4.1.2 Organization } & 67\end{array}$

4.2 The model development $\ldots \ldots \ldots \ldots$. . . . . . 67

$\begin{array}{lll}\text { 4.2.1 Modeling } & 67\end{array}$

4.2 .2 Approach . . . . . . . . . . . . . 72

4.3 Numerical Results . . . . . . . . . . . . . . . . . . . 72

4.3.1 Computer Codes . . . . . . . . . . . . . 73

4.3.2 Validation . . . . . . . . . . . . 73

$\begin{array}{lll}4.4 \text { Summary } & 76\end{array}$

$\begin{array}{lll}5 & \text { Extension, conclusion, and future research } & 78\end{array}$

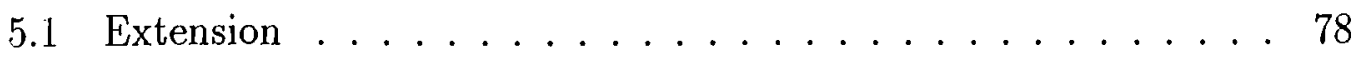

5.1 .1 Line Behavior and Design Issues . . . . . . . 78

5.1 .2 Longer transfer lines $\ldots \ldots \ldots$. . . . . . 80 
5.1 .3 Non-exponential distributions $\ldots \ldots \ldots . \ldots 81$

5.1 .4 Pull-type systems . . . . . . . . . . 82

$5.1 .5 \quad$ General networks . . . . . . . . . . 83

5.2 Conclusion and future research $\quad 84$

A Typical serial lines: Analytical derivations 108

A.1 Two-machine-one buffer system . . . . . . . . . 108

A.2 Three-machine-two-buffer system . . . . . . . . . 113

A.3 Formulations for the descriptions of the system behavior $\ldots . .122$

A.3.1 Atomic model . . . . . . . . . . . . . 122

A.3.2 Two-machine-one-buffer model . . . . . . . . . 123

A.3.3 Three-machine-one-buffer model . . . . . . . . . . . . 124

A.4 Computational results $\ldots \ldots \ldots \ldots \ldots$

A.4.1 Atomic model . . . . . . . . . . . . . 130

A.4.2 Two-machine-one-buffer system . . . . . . . . 133

A.4.3 Three-machine-two-buffer system . . . . . . . . 136

B Two-part type systems: Analytical derivations $\quad 139$

B.1 Two-machine-one-buffer system . . . . . . . . . 139

B.2 Three-machine-two-buffer system . . . . . . . . . 143 


\section{List of Figures}

1.1 Two-Machine Serial Line Production System.

$3.1 T_{\lambda}^{i} \sim \exp (\lambda), T_{\mu_{1}}^{i} \sim \exp \left(\mu_{1}\right) \forall i ; b_{0}=0 \& b_{1}=\infty$.

3.2 Evolution of the stochastic process, $N_{1}(t)$.

$3.3 T_{\lambda}^{i} \sim \exp (\lambda), T_{\mu_{j}}^{i} \sim \exp \left(\mu_{j}\right) \forall i, j=1,2 ; b_{0}=0, b_{1} \geq 0, \& b_{2}=\infty .51$

$3.4 T_{\lambda}^{i} \sim \exp (\lambda), T_{\mu_{j}}^{i} \sim \exp \left(\mu_{j}\right) \forall i, j=1,2,3 ; b_{0}=0, b_{1} \geq 0, b_{2} \geq 0$, $\& b_{3}=\infty \ldots \ldots \ldots \ldots \ldots \ldots \ldots \ldots$

3.5 Mean and Variance vs time: the atomic model . . . . . . . 61

3.6 Mean and Variance vs time: the two-machine model . . . . . 62 .

3.7 Mean and Variance vs time: the three-machine model . . . . . 63

$4.1 T_{\lambda}^{j, i} \sim \exp (\lambda), T_{\mu_{1 j}}^{j, i} \sim \exp \left(\mu_{1 j}\right) \forall i \& j=1,2 ; \mathrm{b}_{0, j}=0 \& b_{1 j}=\infty$ $\mathrm{j}=1,2$.

4.2 Evolution of the stochastic process, $N_{1}(t) \ldots \ldots \ldots 8$

$4.3 T_{\lambda}^{j, i} \sim \exp (\lambda), T_{\mu_{s j}}^{j, i} \sim \exp \left(\mu_{s j}\right) \forall i \& j=1,2, \mathrm{~s}=1,2 ; \mathrm{b}_{0 j}=0$, $b_{1 j} \geq 0, \& b_{2 j}=\infty j=1,2 \ldots \ldots \ldots \ldots \ldots \ldots$

$4.4 T_{\lambda}^{j, i} \sim \exp (\lambda), T_{\mu_{s j}}^{j, i} \sim \exp \left(\mu_{s j}\right) \forall i, j=1,2, \mathrm{~s}=1,2,3 ; \mathrm{b}_{0 j}=0$, $b_{s j} \geq 0, \& b_{3 j}=\infty, j=1,2 \& s=1,2 \ldots \ldots \ldots \ldots \ldots$ 
4.5 Mean and Variance vs time: the atomic model . . . . . . . . 75

4.6 Mean and Variance vs time: the two-machine model . . . . . . 76

4.7 Mean and Variance vs time: the three-machine model . . . . . 77

A.1 Evolution of the stochastic process, $N_{1}(t) \& N_{2}(t) \ldots \ldots 109$

A.2 Evolution of the stochastic process, $N_{1}(t), b_{1}(t), \& N_{2}(t) \ldots \ldots 109$

A.3 Evolution of the stochastic processes $\left.N_{1}(t), b_{1}(t), \& N_{2}(t)\right) . \ldots 110$

A.4 Mean and Variance vs time: the atomic model . . . . . . . . 131 


\section{List of Tables}

2.1 Classification from the applications objective viewpoint . . . 17

2.2 Classification from the viewpoint of performance criteria $\ldots . \quad 19$

2.3 Classification on the basis of the methodology followed $\ldots . .21$

2.4 Assumptions considered in the literature $\ldots \ldots \ldots . \ldots 28$

2.5 Classification from the methodology viewpoint . . . . . . . 34

3.1 The assumptions $\ldots \ldots \ldots \ldots \ldots \ldots \ldots$

3.2 The notation 49

3.3 Analytical results vs state-space representations: the atomic model 59

3.4 Analytical results vs state-space representations: the twomachine model . . . . . . . . . . . . . . . 59

3.5 Analytical results vs state-space representations: the threemachine model . . . . . . . . . . . . . . 60 60

3.6 Analytical results vs simulation: the atomic model . . . . . . . 61

3.7 Analytical results vs simulation: the two-machine model . . . 62

3.8 Analytical results vs simulation: the three-machine model . . . 63

xii 
4.1 The additional assumptions $\ldots \ldots \ldots \ldots 6$

4.2 The additional notation

4.3 Analytical results vs state-space representations: the atomic model 73

4.4 Analytical results vs state-space representations: the twomachine model

4.5 Analytical results vs state-space representations: the threemachine model

4.6 Analytical results vs simulation: the atomic model . . . . . . 75

4.7 Analytical results vs simulation: the two-machine model $\ldots 76$

4.8 Analytical results vs simulation: the three-machine model . . . 77

5.1 Mean and Coefficient of Variation vs time: the two-machine model 79

A.1 Analytical results vs state-space representations: the atomic model130

A.2 Mean and Variance vs time: the atomic model . . . . . . . 131

A.3 Mean and Variance vs time: the atomic model . . . . . . . 132

A.4 Mean and Variance vs time: the atomic model . . . . . . . 132

A.5 Analytical results vs state-space representations: the twomachine model . . . . . . . . . . . . 133

A.6 Mean and Variance vs time: the two-machine model . . . . . . 134

A.7 Mean and Variance vs time: the two-machine model . . . . . . 134

A.8 Mean and Variance vs time: the two-machine model . . . . . 135

A.9 Mean and Variance vs time: the two-machine model . . . . . . 135 
A.10 Analytical results vs state-space representations: the threemachine model . . . . . . . . . . . 136

A.11 Mean and Variance vs time: the three-machine model . . . . 137

A.12 Mean and Variance vs time: the three-machine model $\ldots . .137$

A.13 Mean and Variance vs time: the three-machine model . . . . 138

A.14 Mean and Variance vs time: the three-machine model . . . . 138 


\section{Chapter 1}

\section{Introduction}

A transfer line is a manufacturing system with a very special structure. It is a linear network of service stations or machines $\left(M_{1}, M_{2}, \ldots, M_{n}\right)$ separated by buffer storages $\left(B_{0}, B_{1}, \ldots, B_{n}\right)$. Material flows from outside the system to $B_{0}$, then to $M_{1}$, then to $B_{1}$, and so forth until it reaches $B_{n}$ after which it leaves. Figure 1.1 depicts a two-machine transfer line. The squares represent machines and the triangles represent buffers.

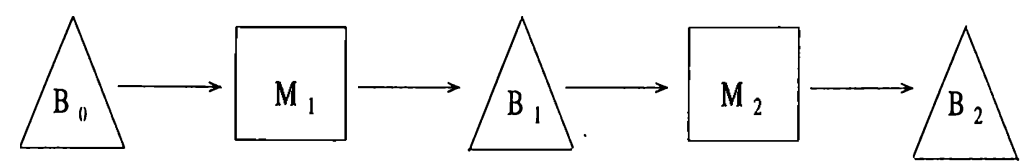

Figure 1.1: Two-Machine Serial Line Production System.

There are two major reasons for studying the transfer lines in this thesis. The first one is that they are of economic importance as they are generally used in high volume production. The second reason is that transfer lines represent the simplest form of the interactions of manufacturing stages and their decoupling by means of buffers (Gershwin [74]).

There is a vast literature on modeling and analysis of the transfer lines. However, most of the results are for steady-state operation, i.e., they pertain to the characteristics of the system of interest as the time from initialization becomes very large and the initial conditions no longer have any effect on 
the system performance. The literature emphasizes this type of analysis because the equations involved are considerably simplified in the limit, and relatively straightforward techniques such as balance equations can then be used. Therefore, they are modeled as Markov chains. However, Markov chain models of such systems are difficult to treat because of their large state spaces and indecomposibility. Besides, such steady-state analyses are inappropriate in many applied situations since the time horizon of operation naturally terminates, steady-state measures of system simply do not make sense. Therefore, in applied queueing theory, it is often important to know, at least approximately, the manner in which a queueing system approaches the steady-state behavior. However, transient results can be quite difficult to obtain, tend to be rather complicated, and are available only for a fairly restricted class of models.

In the literature, almost all the methods deal with steady-state average production rates and steady-state average buffer levels. However, the variance of the production and of the buffer levels during a time period is also important. Today, manufacturers deliver products on a daily or weekly basis. "Informal numerical and simulation experimentation, as well as factory observation, indicate that the standard deviation of weekly production can be over 10 percent of the mean. This implies that, over the course of a year, it is not surprising to see that the production of some weeks can be half that of other weeks. This variability is an inherent characteristic of the manufacturing systems. It is striking that this area is so little appreciated by researchers. Perhaps it is of greater importance now than in the past because of the current emphasis on "just-in-time" production" (Dallery and Gershwin [56]).

A common assumption in the production line literature is that only one type of part is produced (Dallery and Gershwin [56]). This brings a considerable amount of simplification in modeling the system of interest by not taking the batching and setup issues into consideration. However, transfer lines are common in industries such as food, automotive, electronics, and pharmaceutical, among many others (Altiok [11]). By means of the development in the machine design, any machine can act as a single-server 
attending the requests for production of different types of products with its own pool of production orders. At this point, the transient and steady-state analyses of the output process of the transfer lines with multiple-part types gain importance. Moreover, there also exist manufacturing systems exhibiting more general structures such as the assembly systems that can be viewed as the extensions of the flow line structures. Since the funclamental characteristic of assembly systems is that a certain number of units from different input material are assembled to produce one unit of the finished product, the flow lines included in the assembly structure act like the lines with multiple-part types.

This thesis shows how to calculate the mean and the variance of the throughput rate, which we define as the number of parts produced by a transfer line with buffer inventories and exponentially distributed interarrival and processing times per unit time, and then allows to calculate interval estimates for the number of parts produced. These interval estimates provide an operational guide for the production manager. Also, and perhaps more importantly, we are now able to examine the transient behavior of relatively short transfer lines (up to three stages) and derive the distribution of throughput that can be defined as the number of parts produced by a transfer line with buffer inventories and exponentially distributed inter-arrival and processing times. Since transfer lines with high efficiencies and low variances are generally preferred, our results can be used to help design economically feasible transfer lines. Moreover, both the transient and the long run behavior of systems with two-part types can now be determined.

Organization of the thesis is as follows: The next chapter reviews the transfer line literature by emphasizing the studies on the transient and steadystate analyses by the calculation of the first and second order moments of performance measures of interest. Chapter 3 shows how the proposed model is developed. In this chapter, illustrative examples are given and also an experiment is designed in order to compare the results with state-space representations and simulation. Chapter 4 applies the proposed method to the typical transfer lines (up to three stages) with two-part types. Finally, the 
thesis ends with extensions to this research, conclusion, and suggested avenues for future research. 


\section{Chapter 2}

\section{Serial Line Production System:}

\section{A review}

The emphasis in the literature has been on the calculation of the first order moment of the steady-state performance measures. The issues of the transient analysis and the second order moment of the performance measures have been mostly neglected. Therefore, very few papers have attempted to analyze the transient behavior of transfer lines and calculate the variance of the performance measures of interest.

To be able to compare the existing studies, we divide the models into two parts: models for determining the first and second order moments of the performance measures in the steady-state are reviewed in Section 2.1, and the models for analyzing the transient behavior of the transfer lines are reviewed in Section 2.2 .

\subsection{Steady-state behavior}

There are three important review papers on the steady-state analysis of the transfer lines. The first one is by Dallery and Gershwin [56] in which the 
most important models and results of the manufacturing flow line literature are described. These include the major classes of models (asynchronous, synchronous, and continuous); the major features (blocking, processing times, failures and repairs); the major properties (conservation of flow, flow rateidle time, reversibility, and others); and the relationships among different models. Exact and approximate methods for obtaining quantitative measures of performance are also reviewed. The exact methods are appropriate for small systems. The approximate methods, which are the only means available for large systems, are generally based on decomposition, and make use of the exact methods for small systems. The second review is accomplished by Buzacott and Shanthikumar [42] in which design issues of flow lines as well as various types of manufacturing systems such as automatic transfer lines, job shops, flexible machining systems, flexible assembly systems, and multiple cell systems are addressed. Approaches to resolving the design issues of these systems using queueing models are also reviewed. In particular, they show how the structural properties that are recently derived for single and multiple-stage queueing systems can be used effectively in the solution of certain design optimization problems. The third review is by Papadopoulos and Heavey [180] in which a bibliography of material (from 1992 to early 1995) concerned with modeling of production and transfer lines using queueing networks is provided. As well as providing a bibliography of material, a contribution of this paper is also the systematic categorization of the queueing network models based on their assumptions.

Below, we review the recent studies that have been published since 1992 . We emphasize the ones analyzing the systems in terms of their first and second order moments of the steady-state performance measures. The models of interest are explained briefly. Then, the limitations of the existing models are stated.

Hong, Glassey and Seong [104] develop an efficient analytical method for the analysis of an $n$-machine production line with unreliable machines and random processing times. The behavior of the $n$-machine line is approximated by the behaviors of the $(n-1)$ two-machine lines based on the decomposition 
technique proposed by Gershwin [73]. Although there is minor difference between the solutions of this method and the method by Choong and Gershwin [45], simulation and numerical experiments indicate that this method has advantages over the existing ones.

Hendricks [100] develops a technique to analytically describe the output process of a serial production line of $n$ machines with exponential processing time distributions and finite buffer capacities. Extensive exact results are used to examine the effects of line length, buffer capacity and buffer placement on the inter-departure distribution, correlation structure, and variability of the output process of the production line. These results are used to determine the extent to which buffer allocation can be used to control the variability of the output process (and thereby the amount of work-in-process required to downstream subsystems). In addition, insights are provided to help explain why small buffers in production lines are normally adequate.

Hendricks and McClain [101] examine the output process of a serial production line of $n$ machines with general processing time distributions and finite buffer capacities. Simulation is used to observe the effects of line length, buffer capacity, and buffer placement on the inter-departure distribution and correlation structure of the output process of the production line. Results from this analysis is also useful in setting production line design parameters and in determining the extent to which buffer replacement can be used to control the variability of the output process. Additional insights are provided to help us better understand the effects of buffers on tightly coupled production systems.

Papadopoulos [176] considers the throughput rate of multi-station reliable production lines with no intermediate buffers. Processing times at the service stations are independent exponential random variables, possibly with different means. This study is an extension of the work started in Muth [166] to provide an algorithm that allows for the efficient computation of longer lines and to provide results for the non-identical server case. The results provides the distribution function of the holding time at the stations. Furthermore, the 
mean performance of multi-station production lines with and without interstation buffers is determined either exactly by generating the state-space model automatically and solving the system of equations (Papadopoulos [177, 178]) or approximately by decomposition (Tan and Yeralan [228]).

Glassey and Hong [75] develop an efficient method to analyze the behavior of an unreliable $n$-stage transfer line with $(n-1)$ finite inter-stage storage buffers to extend Hong, Glassey and Seong's serial model [104]. They make use of the steady-state output of stage $M_{j}$ in the $n$-stage line rather than the flow rate-idle time relationship. In this way, they show that the analytical approximation compares quite well both in cases of equal production rates and in cases of unequal production rates.

Papadopoulos and O'Kelly [181] develop an exact procedure for the analysis of a production line consisting of single machines linked in series with no intermediate buffers between them. Arrivals occur only at the first queue, which is assumed infinite, according to a Poisson distribution. All processing times and inter-arrival times are assumed to be exponentially distributed. Departures from the system may only occur from the last machine. The feedback case is also included. The exact algorithm gives the marginal probability distribution of the number of units in each machine, the mean queue length and the critical input rate, i.e., the throughput rate of the line.

Heavey, Papadopoulos and Browne [96] examine unreliable multi-station. series production lines. The first station is never starved and the last station is never blocked. The processing times at each station $j$ is Erlang-type distributed with the number of phases allowed to vary for each station. Buffers of nonidentical capacities are also allowed between successive stations, time to failure is assumed to be exponentially distributed, and repair times are considered to be Erlang-type distributed with the number of phases allowed to vary at each station. They propose a methodology for generating the associated set of linear equations. These set of linear equations are solved via the use of the Successive Over-Relaxation (SOR) method with a dynamically adjusted relaxation factor. Referring to the throughput rate of the production lines, 
many numerical results are solved and documented. These exact results are of use for comparison purposes against approximate results which exist in the literature.

Springer [221] proposes a new approximation for estimating the throughput rate and work-in-process inventory of finite-buffer exponential queues in series. The approximation is applied to several sets of previously published test problems and is shown to consistently perform well relative to existing models. In addition, a large simulation experiment is conducted to examine the robustness of the approximation under a wide range of parameter settings.

Frein, Commault and Dallery [69] propose an analytical method for the performance evaluation of closed-loop production lines with unreliable machines and finite buffers. They assume that machines have deterministic processing times but are subject to failures. Failures and repair times are exponentially distributed. They approximate the behavior of this system by a continuous flow model that is analyzed with a decomposition technique, which is similar to that used for (open) production lines. Numerical experiments show that the results provided by this method are in general fairly accurate.

Papadopoulos [179] derives an approximate analytical formula, using the holding time model (HTM) method, for calculating the average throughput rate of a $n$-station production line with exponential service times, manufacturing . blocking, and no intermediate buffers between adjacent stations. The usefulness of the proposed analytical formula relies on the fact that it can handle the (general) case of workstations with different mean processing times - this being the contribution of this work compared against that of Alkaff and Muth [10] - provided a good estimation of some coefficients involved is being made.

Finally, Dinçer and Donmez [59] propose a Markov model for transfer lines consisting of $n$ reliable machines with Erlang processing times and finite buffers. The arrivals to the system is Poisson distributed. A program coded in $\mathrm{C}$ which is capable of solving the Markov model of a three-machine transfer line is also developed. They calculate the mean throughput rate, machine utilization, 
expected value of WIP level, and also the variance of WIP.

The performance measures of almost all the studies correspond to the steady-state average production rates and steady-state average buffer levels. However, the essence of transfer lines is their variability and this issue has been entirely neglected. As far as we are aware of, there are only two published papers that deal with the calculation of the variance of the behavior of a transfer line over a limited period: Lavenberg [134] and Miltenburg [155].

Lavenberg [134] derives an expression for the Laplace-Stieltjes transform of the steady-state distribution of the queueing time for the $M / G / 1$ finite capacity queue. The derivation proceeds in terms of a related two-stage closed cyclic queueing network. The resulting expression is a rational function of the steadystate probabilities of the embedded Markov chain at departure epochs and of the Laplace-Stieltjes transform of the service time distribution. The expression can be differentiated readily in order to obtain higher order moments of the steady-state queueing time. He concludes that the higher order moments about the origin of the steady-state queueing time for the $M / G / 1$ finite capacity queue does not depend on the service time distribution only through its first $m+1$ moments as is the case for the $M / G / 1$ infinite capacity queue. Also, they show that the mean queueing time does not necessarily increase as the variance of the service time increases.

Miltenburg [155] models the transfer line with an ergodic Markov chain. He presents a procedure for calculating the variance of the number of units produced by the transfer line during a period of length $T$ cycles. It makes use of the fundamental matrix for a Markov chain and obtains a representation $\left(c_{a b}\right)$ for the limiting covariance for the number of times state $a$ and state $b$ are each occupied over $T$ cycles. Then, it is concluded that the representation of $c_{a a}$ is the limiting variance for the number of times state $a$ is occupied over $T$ cycles. As the number of cycles $T$ gets large, the influence of the initial starting state diminishes. Finally, they use the mean and the variance to construct an interval estimate for the number of units produced during a shift. This interval estimate is an operational guide for the production manager. 
There are also four working papers by $\mathrm{Ou}$ and Gershwin [175], Gershwin [214], Wu [245], and Tan [227]:

$\mathrm{Ou}$ and Gershwin [175] derive a closed form expression for the variance of the lead time distribution of a two-machine transfer line with a finite buffer and Gershwin [214] analyzes the variance of a tandem production system.

Wu [245] contributes to two related problem categories for transfer lines. First, algorithms are developed to calculate the variance of the number of departures at a fixed time interval from transfer lines with finite buffer inventories. Both tandem queueing network models and discrete-time models with breakdowns and repairs are taken into consideration while the algorithms of interest are based on existent decomposition algorithms for calculating the throughput rate. Second, the variance calculation is incorporated into planning and designing procedures of transfer lines, and also basic issues such as due time performance, quota setting and characterization of departure process are considered.

Finally, Tan [227] determines analytically the variance of the throughput rate of a $n$-machine production line with no intermediate buffers and timedependent failures. The analytical method, which yields a closed-form expression for the variance of the throughput rate, is based on determining the limiting variance of the total residence (sojourn) time in a specific state of an irreducible recurrent Markov process from the probability of visiting that state at an arbitrary instant given an initial state. The corresponding procedure can also be applied to determine the variance of the throughput rate of various arrangements of workstations including series, parallel, series-parallel systems provided that the instantaneous availabilities of these systems can be written explicitly.

The limitations of these analytical models can be stated as follows:

- The main missing area is that of variability. As has been indicated, there are only two published papers that deal with the variance of the output of a transfer line that only treat two-machine lines, and obtain results 
that are difficult to use and to understand intuitively. It is not clear how to extend the results of these papers either exactly or approximately. Therefore, this class of problems should be high on the research agenda of the field.

- Almost all the models in the transfer line literature make the assumption that the first machine never gets idle and the last machine never gets blocked. However, the general case in the real-world settings is that both the input and the output buffer storages are of finite size.

- Methods that can handle more general distributions of inter-arrival time, service rates, times to failure and times to repair other than exponential (or geometric) distribution, should also be developed.

- Approximation methods for flow lines with parallel machines will prove useful.

- Other issues include multiple-part types, routing, control, correlated failures, and systems with machines of different types. An example of the latter is a system with batched continuous material so the upstream portion of the line is continuous and the downstream portion is synchronous. Another example is a line consisting of some automated stations and some manual stations. A portion of the line might be synchronous while the rest is asynchronous.

- Almost all the systems in the literature are modeled as puish-type systems. However, attention should be given to the finished-product inventory by modeling the systems under consideration as pull-type systems. This area and the general issue of just-in-time production are pertinent to the transfer line literature and will prove to be important in the near future.

- There is already sufficient number of works in the literature that deals with the allocation of storage or machine capacity, particularly based on optimization methods. It would however be of interest to develop simple rules of thumb for resource allocation and to prove bounds on their distance from optimality. 


\subsection{Transient behavior characterization}

In this section, the most important models and results of transient behavior of transfer line literature are described. Furthermore, a unifying classification scheme is developed for the transient analysis of the serial lines with respect to design and operating characteristics.

The first section gives a brief review on how the work on the transient analysis of serial lines began and evolved in the late and early 1950s and early 1980s. The next one presents a topology of the recent serial line production system literature beginning from the mid $80 \mathrm{~s}$. The succeeding sections will review the recent literature along the dimensions of this topology.

\subsubsection{Evolution of the work on the transient analysis of serial lines}

Much of the work on the transient behavior of serial lines is conducted in the late 1950s and early 1960s. Efforts are mainly devoted to $M / M / 1$ queue or its variations. See, for example, Bailey $[20,21]$, Saaty [204], Bhat and Sahin [28], Heathcote and Winer [95], Morisaku [161], and the references cited therein. The transient behavior of $M / G / 1$ queue (and more general models) is also studied extensively, so that there is a substantial literature including the early papers by Kendall [121, 122], Takács [223, 225], Benes̃ [23], and Keilson and Kooharian [117]; the advanced books by Takács [224, 226], Bene $\tilde{s}$ [24], Prabhu [187]. However, the most important observation has been the following: for many serial production systems, "the rate at which a queue converges to its steady-state characteristics, independently of the system's initial state, eventually becomes (for large values of time $t$ ) dominated by an exponential term of the form $\exp (-t / \tau)$ where $\tau$ is a characteristic of the queueing system" (Odoni and Roth [172]). Karlin and McGregor [113] first prove this result for finite-state and infinite-state birth-and-death processes. It is subsequently extended to $M / G / 1$ and $G I / M / 1$ queueing systems by Kendall 
[123], to $G I / E_{r} / 1$, and $E_{r} / G / 1$ systems by Vere-Jones [233], and, finally, to $G I / G / 1$ systems, with some minor restrictions, by Cohen [47]. The latter also describes a procedure for obtaining the characteristic time constant $\tau$, the relaxation time, for $G I / G / 1$ systems (Section III.7.3 of Cohen [47]). The concept of relaxation time for queues is first introduced by Morse [163, 164]. In another noteworthy work, Heathcote [94] discusses the concept of exponential convergence within the general context of Markov chains and of random walks.

By 1970s, "work on the transient behavior of serial line production systems has concentrated increasingly on approximations and on numerical techniques. This change in emphasis is undoubtedly motivated, in part, by the analytical complexity of the problems involved in a theoretical analysis, amply demonstrated in the body of work that has already been cited (Gross and Harris [89], Heyman and Sobel [103], and Keilson [116])" (Odoni and Roth [172]). There appears two principle methods that are the randomization technique introduced by Grassman [86] and numerical integration method of the Kolmogorov differential equations (Gross and Harris [89], section 7.3.2 and references therein).

In general, analytical investigations of the transient period of serial line production systems are rare: "Newell [171] refers briefly to the problem of time to steady-state in his work on the diffusion approximation of $G I / G / 1$ queueing systems under heavy traffic. He obtains a closed form expression and proposes an order of magnitude estimate of the time required for the transient effects to become negligible. Barzily and Gross [20] examine the transient response of the stationary, finite-source $M / M / n$ queueing system. Their particular concern is to measure the amount of time that the system takes to reach equilibrium. They compare four measures of the distance of the system from steady-state. Their report contains some interesting intuitive observations as well as numerical examples, but does not progress to the point of specifying a procedure for predicting the time to equilibrium. Finally, a paper by Marks [150] applies regression techniques to study the manner in which the time to steady-state depends on the traffic intensity. He fits linear, quadratic, and parabolic regression models to simulation results of an infinite-capacity, single 
queue $M / M / m$ system. The work depends on the accurate determination of the point, $t_{\infty}$, after which the system is in equilibrium. This is a difficult problem in simulation, and Marks' choice of $t_{\infty}$ seems rather arbitrary" (Odoni and Roth [172]).

"Much important work is done on approximate or exact numerical solution techniques that can be used to investigate the transient behavior (e.g., Koopman [192], Neuts [170], Moore [159], Rider [192], Mori [160], Collings and Stoneman [48], Chang [44], Grassman [86, 87], Kotiah [133], Grassman and Servranckx [88], and Rothkopf and Oren [203])" (Odoni and Roth [172]).

The transient behaviors of the $M / G / 1$ and $G I / M / 1$ queues are also studied in detail by Kingman [124], Heyman [102], Ott [173, 174], Harrison [93], Middleton [154], Prabhu [188], Stanford, Pagurek, and Woodside [222], Asmussen [13], and Neuts [169]. In another noteworthy study, Ramaswami [191] characterizes the busy period of a $G / P H / 1$ queue using the matrix geometric approach. A good basic reference is Kleinrock [125].

\subsubsection{A topology}

The recent literature on the transient analysis of the serial line production systems addresses the problems at many levels of detail using various techniques. A convenient classification, which captures the essential differences in the publications, is along the following dimensions:

(1) Applications objective. Serial line production systems can be classified in many different ways (Dallery and Gershwin [56], Buzacott and Shanthikumar [42]). One convenient way of classification is made according to the objective of the study: performance evaluation, determination of bounds on the system performance measures, and transient period determination.

(2) System assumptions considered. Although a serial line production system is said to represent the simplest form of the interactions of manufacturing 
stages and their decoupling by means of buffers, it is a complex system with a number of assumptions characterizing the real-life settings: line length, arrival type, queue discipline, multiple servers, and multiple-part types. Very few authors have addressed all of this complexity.

(3) Methodology used in resolving the problems. The literature can be distinguished on the basis of the technique employed by the authors. Most authors have cast the problems into a mathematical model. Some authors have investigated heuristics. A few authors have used simulation-, based techniques.

In the following sections we present the review of available literature from the above viewpoints.

\section{Applications objective}

Based on the objective in the transient analysis of serial line production systems, the related literature can be classified as follows:

(1) Performance evaluation.

(2) Determination of bounds on the system performance measures.

(3) Transient period determination.

The classification of the literature based on the applications objective is given in Table 2.1.

\section{Performance evaluation}

The impact of a policy decision or design change on the behavior of a system may be either measured by observation or estimated using a methodology. This is usually called performance evaluation. The prerequisite of performance 
Table 2.1: Classification from the applications objective viewpoint

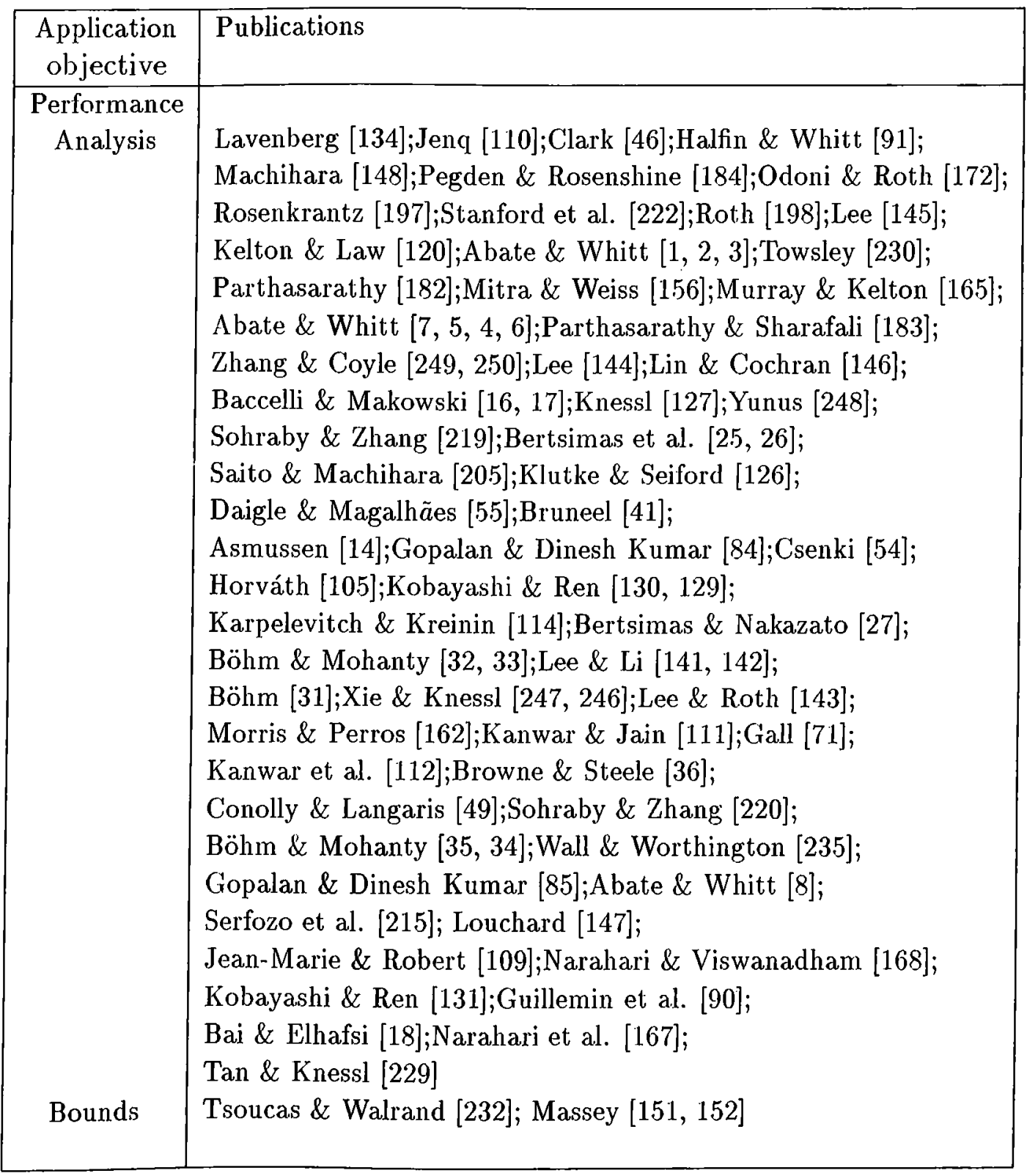

evaluation. The prerequisite of performance evaluation is to decide on the performance criteria, which may be a single measure or a set of measures. Typical measures in the serial line production system literature are output rates or throughput rate (number of parts produced per unit time), output time (expected time a part leaves the system), queue length (number of parts in the queues), congestion level (number of parts in the system), workload (unfinished work), utilization (percentage of time a machine is busy), idle 
Table 2.1: Classification from the applications objective viewpoint (cont'd)

\begin{tabular}{|c|c|}
\hline $\begin{array}{c}\text { Application } \\
\text { objective }\end{array}$ & Publications \\
\hline $\begin{array}{l}\text { Transient } \\
\text { period }\end{array}$ & $\begin{array}{l}\text { Moeller \& Kobayashi [157];Wilson \& Pritsker [244, 243]; } \\
\text { Gafarian et al. [70]; Kelton [118];Whitt [238]; } \\
\text { Schruben [210, 211];Welch [236, 237]; Law \& Kelton [138]; } \\
\text { Adlakha \& Fishman [9];Schruben et al. [213]; } \\
\text { Heidelberger \& Welch [99];Kelton \& Law [119]; Law [136]; } \\
\text { Law \& Kelton [139]; Snell \& Schruben [218];Roth [198]; } \\
\text { Ripley [194]; Whitt [240, 239];Law \& Kelton [140];Roth [199]; } \\
\text { Glynn \& Heidelberger [77];Roth \& Josephy [200];Robinson [195]; } \\
\text { Asmussen et al. [15]; Raatikainen [190];Glynn [76]; } \\
\text { Whitt [241, 242];Glynn \& Heidelberger [79, 78];Jacobson [107]; } \\
\text { Roth \& Josephy [201];Rosetti \& Delaney [197]; } \\
\text { Gallagher et al. [72];Deler \& Sabuncuoğlu [58] }\end{array}$ \\
\hline
\end{tabular}

period (percentage of time a machine is idle), blocking probability (percentage of time a machine is blocked), queueing time (average time a part spends in the queue), and sojourn time (average time a part spends in the system). The classification of the literature based on the performance criteria is provided in Table 2.2. The impact of a number of changes on the system's behavior in terms of these measures usually requires an analysis of the system. In most cases this analysis is carried out with the help of a mathematical or a computer simulation model of the system.

\section{Bounds on the performance measures}

When tractable techniques become insufficient in analyzing the model of a system, numerical techniques, simulation, or approximations are often used. In the lack of exact results, bounds are useful to test approximations, or they are simply the only information that can be obtained for certain measures. Furthermore, a bound may exhibit similar behavior to that of the measure itself. Then, at times it may be sufficient to observe the behavior of the bounds for design purposes. 
Table 2.2: Classification from the viewpoint of performance criteria

\begin{tabular}{|c|c|}
\hline Performance criteria & Publications \\
\hline \multirow[t]{5}{*}{ Throughput Rate } & \\
\hline & Towsley [230];Daigle \& Magalhães [55]; \\
\hline & Morris \& Perros [162];Böhm \& Mohanty [35]; \\
\hline & Narahari \& Viswanadham [168];Bai \& Elhafsi [18]; \\
\hline & Narahari et al. [167] \\
\hline Output time & Klutke \& Seiford [126] \\
\hline \multirow[t]{6}{*}{ Queue length } & Odoni \& Roth [172];Roth [198];Massey [152]; \\
\hline & Abate \& Whitt $[3,7] ;$ Bertsimas et al. [26]; \\
\hline & Karpelevitch \& Kreinin [114]; Lee \& Li $[141,142]$ \\
\hline & Bertsimas \& Nakazato [27]; Lee \& Roth [143]; \\
\hline & Morris \& Perros [162]; Jean-Marie \& Robert [109]; \\
\hline & Sohraby \& Zhang [220] \\
\hline \multirow{2}{*}{$\begin{array}{l}\text { Congestion } \\
\text { level }\end{array}$} & \\
\hline & $\begin{array}{l}\text { Kelton \& Law [120]; Baccelli \& Makowski }[16,17] \text {; } \\
\text { Lin \& Cochran [146]; Xie \& Knessl [247, 246]; } \\
\text { Abate \& Whitt [8]: Tan \& Knessl [229] }\end{array}$ \\
\hline Workload & Tan \& Knessl [229] \\
\hline Utilization & $\begin{array}{l}\text { Bertsimas et al. [26]; Gopalan \& Dinesh Kumar [84]; } \\
\text { Browne \& Steele [36]; Gopalan \& Dinesh Kumar [85] }\end{array}$ \\
\hline Idle period & $\begin{array}{l}\text { Conolly \& Langaris [49]; Sohraby \& Zhang [220]; } \\
\text { Gopalan \& Dinesh Kumar [85] }\end{array}$ \\
\hline $\begin{array}{l}\text { Blocking } \\
\text { probability }\end{array}$ & $\begin{array}{l}\text { Mitra \& Weiss [156]; Yunus [248]; Morris \& Perros [162]; } \\
\text { Saito \& Machihara [205];Gopalan \& Dinesh Kumar [85] }\end{array}$ \\
\hline \multirow[t]{3}{*}{ Queueing time } & Lavenberg [134];Murray \& Kelton [165]; \\
\hline & $\begin{array}{l}\text { Karpelevitch \& Kreinin [114];Bertsimas \& Nakazato [27]; } \\
\text { Serfozo et al. [215];Narahari \& Viswanadham [168]; }\end{array}$ \\
\hline & Narahari et al. [167]; Lin \& Cochran [146]; \\
\hline Sojourn time & Csenki [54]; Gall [71] \\
\hline
\end{tabular}

In the literature, the only system for which a family of stochastic bounds are derived is the Jackson network with single-server nodes. Such a network, $(M / M / 1)^{n}$, is a collection of $m M / M / 1$ queues where the $i^{t h}$ one has (external) arrival rate $\lambda_{i}$ and service rate $\mu_{i}$. Jackson [106] derives the joint queuelength distribution for the steady-state. Massey [151] obtains a stochastic upper bound for the transient joint-queue length distribution of $(M / M / 1)^{n}$ as a product of distributions for $M / M / 1$ queues. That each node can be bounded 
above by an $M / M / 1$ queue is not surprising and this can be proved through path-wise arguments. What is not as intuitive is the claim that the product of these bounds serve as an upper bound for the joint tail distribution of the queue-length process. Tsoucas and Walrand [232] give an alternate proof for the results of Massey [151] using stochastic differentials generalizing to similar networks that are non-Markov. Massey [152] generalizes the bound of Massey [151] for $(M / M / 1)^{n}$. What Massey [152] proves is that if the set $\{1,2, \ldots, n\}$ is partitioned into disjoint index sets $I$ and each subnetwork is bounded by an $(M / M / 1)^{I}$ network, then the product of these bounds is a bound for the transient distribution of the entire $(M / M / 1)^{n}$ system. However, this type of bound cannot be extended to a sample-path comparison between the two processes.

It would be of interest to prove bounds on the performance criteria as well as their distance from optimality for a variety of serial line production systems (Dallery and Gershwin [56]).

\section{Transient period determination}

The steady-state analyses of serial line production systems are inappropriate in some real-life situations. When the time horizon of operation naturally terminates, steady-state measures of system simply do not make sense due to frequent changes in the system. Therefore, it is important to know, at least approximately, how long it takes for a serial line production system to effectively reach steady-state. There are a number of methods suggested in the literature for choosing the transient period. A classification of the related literature based on the methodology followed is given in Table 2.3.

We start with estimation procedures that focus on deletion (or truncation) of some initial portion of the run where the transient bias is most severe. These methods attempt to identify at what point in the output process the system reaches to steady-state and data are retained only from this point on. There are six fundamental approaches for addressing the problem (Law and Kelton $[140])$ : 
The first one is the replication/deletion approach for obtaining a point estimate and confidence interval for the steady-state mean. The simplest and most general technique is a graphical procedure due to Welch $[236,237]$. However, the application of Welch's procedure turns out to be rather difficult in practice when the required number of replications are relatively large and the process under consideration is highly variable.

Table 2.3: Classification on the basis of the methodology followed

\begin{tabular}{|c|c|}
\hline Methodology & Publications \\
\hline Estimation Procedures & \\
\hline Replication/deletion & Welch $[236,237]$ \\
\hline Batch means & Law [135];Sargent et al. [206]; \\
\hline & $\begin{array}{l}\text { Meketon \& Schmeiser [1.53];Schmeiser [207]; } \\
\text { Law \& Carson [137];Bischak [29] }\end{array}$ \\
\hline Autoregressive & Fishman $[63,64,68]$; Schriber \& Andrews [209] \\
\hline Spectral & $\begin{array}{l}\text { Fishman [62];Raatikainen }[190,189] \text {; } \\
\text { Law \& Kelton [139];Heidelberger \& Welch [99, 97, 98]; }\end{array}$ \\
\hline Regenerative & $\begin{array}{l}\text { Fishman [65, 66]; Crane \& Iglehart }[50,51,52] \\
\text { Crane \& Lemoine [53]; Fishmann }[67]\end{array}$ \\
\hline Standard time series & $\begin{array}{l}\text { Schruben [211, 212]; Schruben et al. [213]; } \\
\text { Goldsman \& Schruben [82]; Goldsman \& Meketon [81]; } \\
\text { Duersch \& Schruben [60]; Sargent et al. [206]; } \\
\text { Goldsman \& Schruben [83]; Glynn \& Iglehart [80] }\end{array}$ \\
\hline $\begin{array}{l}\text { Analytical queueing } \\
\text { approximations }\end{array}$ & $\begin{array}{l}\text { Whitt [240, 242]; Asmussen et al. [15]; } \\
\text { Rossetti \& Delaney [197]; Glynn [76] }\end{array}$ \\
\hline $\begin{array}{l}\text { Method of independent } \\
\text { replications }\end{array}$ & Glynn \& Heidelberger $[77,79,78]$ \\
\hline Heuristics & $\begin{array}{l}\text { Odoni \& Roth [172]; Roth \& Josephy }[200,201] \\
\text { Roth [199]; Robinson [195] }\end{array}$ \\
\hline Harmonic analysis & Jacobson $[107]$ \\
\hline $\begin{array}{l}\text { Bayesian Technique } \\
\text { (MMAE) }\end{array}$ & Gallagher et al. [72] \\
\hline
\end{tabular}

The method of batch means, like the replication/deletion approach, seeks to obtain independent observations. However, since the batch-means method is based on a single run, it has to go through the transient period only once. Law [135] shows that the sample mean of the first delays for the $M / M / 1$ queue 
is approximately normally distributed if the length of the batches is large. Law and Carson [137] develop a sequential procedure based on batch means and a relative-error stopping rule. There have been several variations of batch means proposed in the literature. Meketon and Schmeiser [153] introduce the method of overlapping batch means. Bischak [29] studies the idea of weighted batch means. Other papers that discuss batch means, in general, are Schmeiser [207] and Sargent,Kang, and Goldsman [206].

The autoregressive method, developed by Fishman $[63,64,68]$, uses the estimates of the autocorrelation structure of the underlying stochastic process to obtain an estimate of the variance of the sample mean and ultimately to construct a confidence interval for the steady-state mean. A major concern in using this approach is whether the autoregressive model provides a good representation for an arbitrary stochastic process. Schriber and Andrews [209] give a generalization of the autoregressive method that allows for movingaverage components as well.

The most complicated technique, requiring a fairly sophisticated background on the part of the analyst is the spectral approach. Moreover, there is no definitive procedure for choosing the value of the weighting function. Detailed discussions of spectral methods can be found in Heidelberger and Welch $[99,97,98]$. The recent studies using the spectral method are accomplished by Raatikainen [189] in which a procedure is proposed for estimating quantiles based on the $P^{2}$ algorithm of Jain and Chlamtac [108], which does not require storing and sorting the observation. In another study, Raatikainen [190] combines the spectral method introduced by Heidelberger and Welch [97] and the method of independent replications for run length control in parallel simulation. The objective is to determine whether a fixed number of independent replications executed in parallel and the spectral method can provide estimates that are accurate enough. Results from extensive simulation experiments indicate that the method is an attractive way of using parallelism in networks of 5-10 workstations. 
The regenerative approach is an altogether different approach to simulation and thus leads to different approaches to constructing a confidence interval for the steady-state mean. This method is developed simultaneously by Crane and Iglehart [50, 51, 52] and by Fishman [65, 66]. In another study, Fishman [67] develops a procedure based on the regenerative method and on absolute-error stopping rule. A more complete discussion of the regenerative method can be found in Crane and Lemoine [53].

The last approach to be mentioned is the standard time series approach. The major source of error for standard time series is choosing the batch size too small (see Goldsman and Schruben [83] for details). It should be noted that this approach is based on the same underlying theory as Schruben's test [211]. In this important paper, Schruben [211] develops a very general procedure based on standard time series and tests his procedure on several stochastic models and finds that it has high power in detecting initialization bias. A variation of this initialization-bias test is given by Schruben, Singh \& Tierney [213]. Additional references for standard time series, including alternative confidence-interval formulations, are Goldsman and Schruben [82], Goldsman and Meketon [81], Duersch and Schruben [60], Sargent, Kang, and Goldsman [206], Goldsman and Schruben [83], and Glynn and Iglehart [80].

A detailed description and comparison of these estimation procedures can be found in Wilson and Pritsker [244, 243], Gafarian, Ancker, and Morisaku [70], Law [136], Snell and Schruben [218], and Law and Kelton [138, 139, 140]. A nicely written overview of simulation methodology that includes the analysis of output data is also done by Ripley [194] .

We secondly consider analytical queueing approximation. Whitt [240] uses the exponential approximations to determine the initial portion to delete. Preliminary estimates of required run lengths are estimated by calculating the asymptotic variance and the asymptotic bias of the sample mean in the Markov model. In another study, Whitt [242] reviews the positive recurrent potential theory, by which the asymptotic variance, the second-order asymptotic of the variance, and the asymptotic bias of the sample mean of a function of an ergodic 
Markov process are expressed in terms of solutions of Poisson's equations, giving special attention to continuous-time Markov chains. He provides explicit formulas for birth-and-death processes, diffusion processes, and recursive computational procedures for skip-free chains that can be used to design simulation experiments after approximating the stochastic process of interest by one of the elementary Markov processes under consideration. Asmussen, Glynn, and Thorisson [13] are concerned with the question of existence and construction of stationarity detection times and suitable generalizations. The algorithms they develop are intended primarily to establish the boundaries of what is theoretically possible rather than as proposals to the practical simulation analyst as how to eliminate initial bias in real-world simulations. This study indicates that one must take advantage of a priori knowledge of the tail behavior of the regenerative cycle-length random variable in a regenerative setting. Rossetti and Delaney [197] investigate the use of analytical queueing approximations to assist in mitigating the effects of the initial transient period in steady-state $G I / G / n$ queueing simulations. The result of their methodology is a less biased and less variable estimator of the expected waiting time in the queue. Glynn [76] reports a new finding pertaining to the transient period detection test proposed by Schruben [211]; he develops asymptotics that are suggestive of the types of initial transients that the test is capable of detecting.

Glynn and Heidelberger [77] consider the method of independent replications with initial transient deletion for generating confidence intervals for steady-state quantities. To produce intervals with good convergence characteristics, the relative growth rates of the number of replications, the length of each replication, and the deletion period are controlled, and also critical rates for these parameters are determined. Besides, they provide some theoretical insight into the problem of initial transient deletion in the multiple replicate steady-state simulation setting. In another study, Glynn and Heidelberger [79] investigate theoretical properties of a simple method for using parallel processors in discrete event simulations: running independent replications on multiple processors and averaging the results at the end of the runs. Specifically, the problem of estimating steady-state parameters 
from such an experiment is considered. Glynn and Heidelberger [78] are also concerned with using the method of parallel replications for estimating steadystate performance measures of queueing network simulation experiments. Their results indicate that a non-standard estimation procedure in the context of steady-state simulation, a ratio estimator, is required on highly parallel machines. The experiments also show that use of the ratio estimator is advantageous even on machines with only a moderate degree of parallelism. Actually, the results of this paper extend those of Glynn and Heidelberger [77] and Glynn and Heidelberger [79].

An alternative approach exploits the characteristics of the underlying theoretical queueing model in an attempt to determine which portion of the simulation data to retain. This technique, the relaxation time heuristic, is a technique for reducing initialization bias in Monte-Carlo simulation of stationary, infinite-capacity, Markovian queueing systems. It is based on system relaxation times first introduced in an $M / M / 1$ settings by Roth and Rutan [202] and further studied by Blanc and Van Doorn [30]. For simulation of transient behavior, this technique may be used to estimate the effective length of the transient period (see Odoni and Roth [172] for greater detail). This is verified by Roth and Josephy [200] for $M / E_{r} / 1$ and $E_{r} / M / 1$ models. Roth and Josephy [201] also apply and evaluate the relaxation time heuristic for estimation of the steady-state expected number of customers in $M / E_{r} / 1$ and $E_{r} / M / 1$ queueing systems. Roth [199] takes a similar approach to validate the technique for estimating the steady-state expected number of customers in $M / M / n$ queueing systems. In another study, Robinson [195] proposes a method that enables a simulation user to select a suitable run length for a nonterminating steady-state simulation. A heuristic technique is used to enable both expert and non-expert users to determine a suitable run length.

Jacobson [107] quantifies the initial transient effect on the harmonic analysis and finite difference gradient estimators. Four additive initial transient models are developed. 
Gallagher, Bauer, and Maybeck [72] propose an algorithm for determining the appropriate initial-data truncation point for univariate output. The technique entails averaging across independent replications and estimating a steady-state output. A Bayesian technique called Multiple Models Adaptive Estimation (MMAE) is applied to compute a time varying estimate of the output's steady-state mean.

The review of the existing studies for the transient period analysis (Table 2.3) of the serial line production systems indicates that almost all the researchers in this area have dealt with improving the efficiency and accuracy of the solution techniques instead of using their results to draw conclusions on general attributes of transient behavior. There are only few studies in the literature filling this gap to an extent, for example Deler and Sabuncuoğlu [58] examine the effects of the factors (e.g., arrival, service, failure, and repair rates, buffer allocation, distribution type of the service, failure, and repair times, the number of servers at each station, and the number of part types in the system) on the length of the transient period of the output process of the serial line production systems as well as characterize approximately the general form of some transient effects based on empirical observations. However, this study expects the future researchers to provide a consistent theoretical framework for their empirical observations. In addition to developing a better theoretical understanding of transient behavior, there are two other general areas to which their empirical results can be extended. One is to obtain similar experience with other types of manufacturing systems. The second area for extensions is the use of results such as those to develop inexpensive computational techniques for approximating the behavior of manufacturing systems with complicated state descriptions.

\section{System assumptions considered}

What makes a serial line production system to represent the simplest form of the interactions of manufacturing stages and their decoupling by means of buffers are a number of simplifying assumptions on the features such as line 
length, arrival type, queue discipline, multiple servers, and multiple-part types. Most of the results in the literature are based on the following assumptions.

The first machine is never starved and the last machine is never blocked. All the random variables (processing times, uptime, downtime) are independent random variables. The setup time and the transfer time through the buffers is negligible. The failures are single-machine failures and either time dependent or operation dependent failures. When a failure occurs, the part stays on the machine; it can be reworked when the machine is up again (i.e., there is no scrapping of parts); the work resumes exactly at the point it stops. inter-arrival times, processing times, times to failure, and times to repair of machines are generally assumed to be exponentially distributed when time is continuous, geometrically distributed when time is discrete.

Based on this consideration, Table 2.4 depicts a classification on the basis of assumptions considered in the available literature.

One of the features that make the real-life serial line production systems difficult to deal with is the existence of parallel servers. Systems are built with machines in parallel for two reasons: either to achieve a greater production rate or to achieve a greater reliability. The first case is often observed when some operation is inherently much slower than the others. The second case is encountered when some machine is much less reliable than the others. Grassman [87] considers the transient and steady-state results for two parallel queues. Murray and Kelton [165] also study the time dependent behavior of a system consisting of a single queue feeding two servers who work in parallel, the $M / E_{r} / 2$ queueing system. The probabilistic structure of the transient $M / E_{r} / 2$ queue is derived in discrete time where $E_{r}$ denotes a $r$-Erlang distribution. This queue has a two-dimensional state-space. Expressions for the expected delay in queue are formulated in terms of transition probabilities. It is shown that the results are in close agreement with previously published results which are solely for single dimensional state-space systems. Sharma, Ravichandran, and Dass [217] analyze the transient behavior of cold and warm standby systems as well as parallel redundancy. Specifically, for multiple-unit reliability system 
Table 2.4: Assumptions considered in the literature

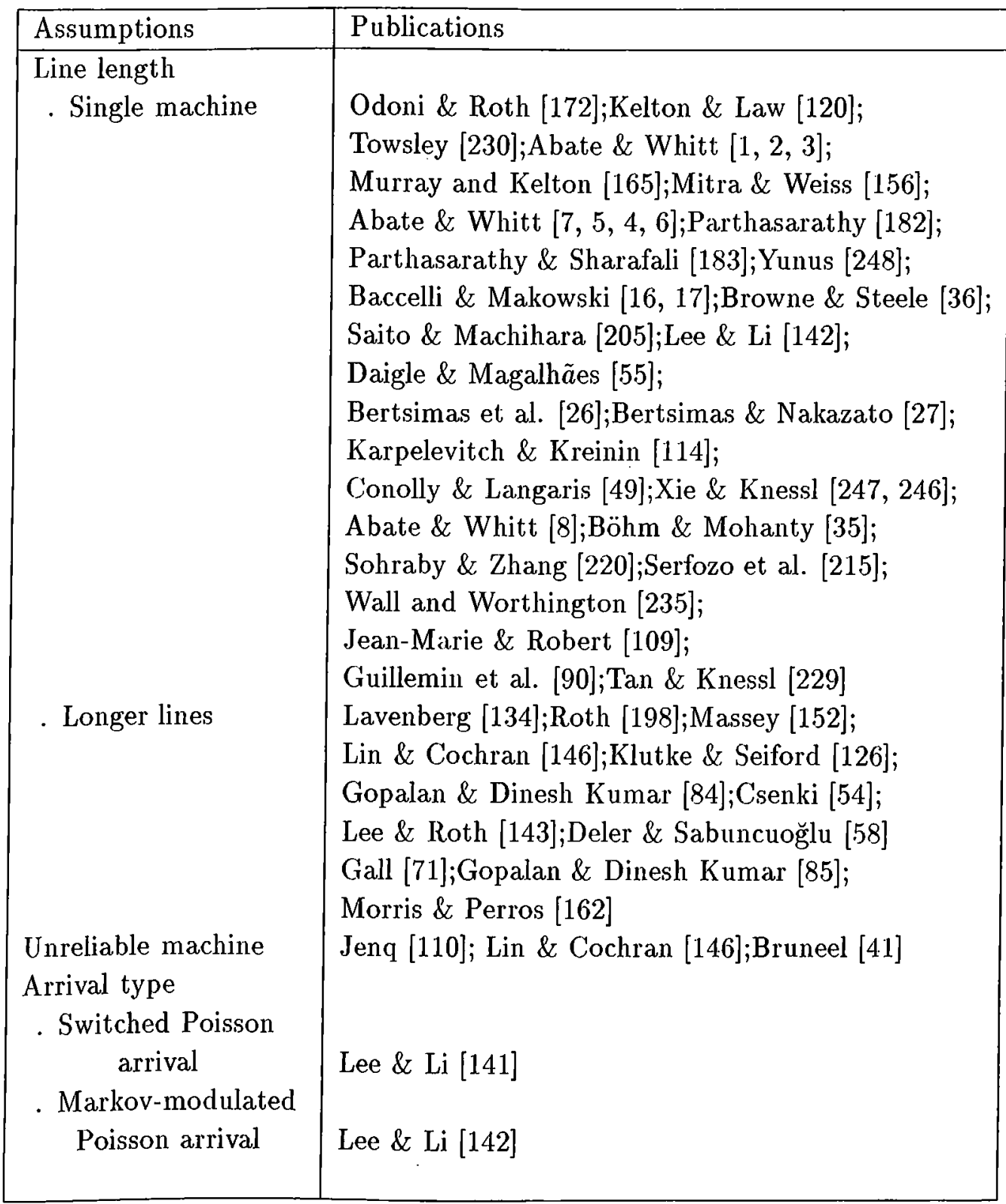

the reliability and availability functions are derived in an explicit form. The stationary availability and mean time to system failure are deduced from the main results as special cases. Duyn Schoute and Wartenhorst [208] also consider the transient analysis of a two-unit standby system with Markovian degrading units. They derive the explicit expressions for the Laplace transforms of the up- and down-periods of this system, which provide insight in the availability of the system and which can be used to obtain approximations for the interval availability distribution. An iterative numerical procedure for the 
Table 2.4: Assumptions considered in the literature (cont'd)

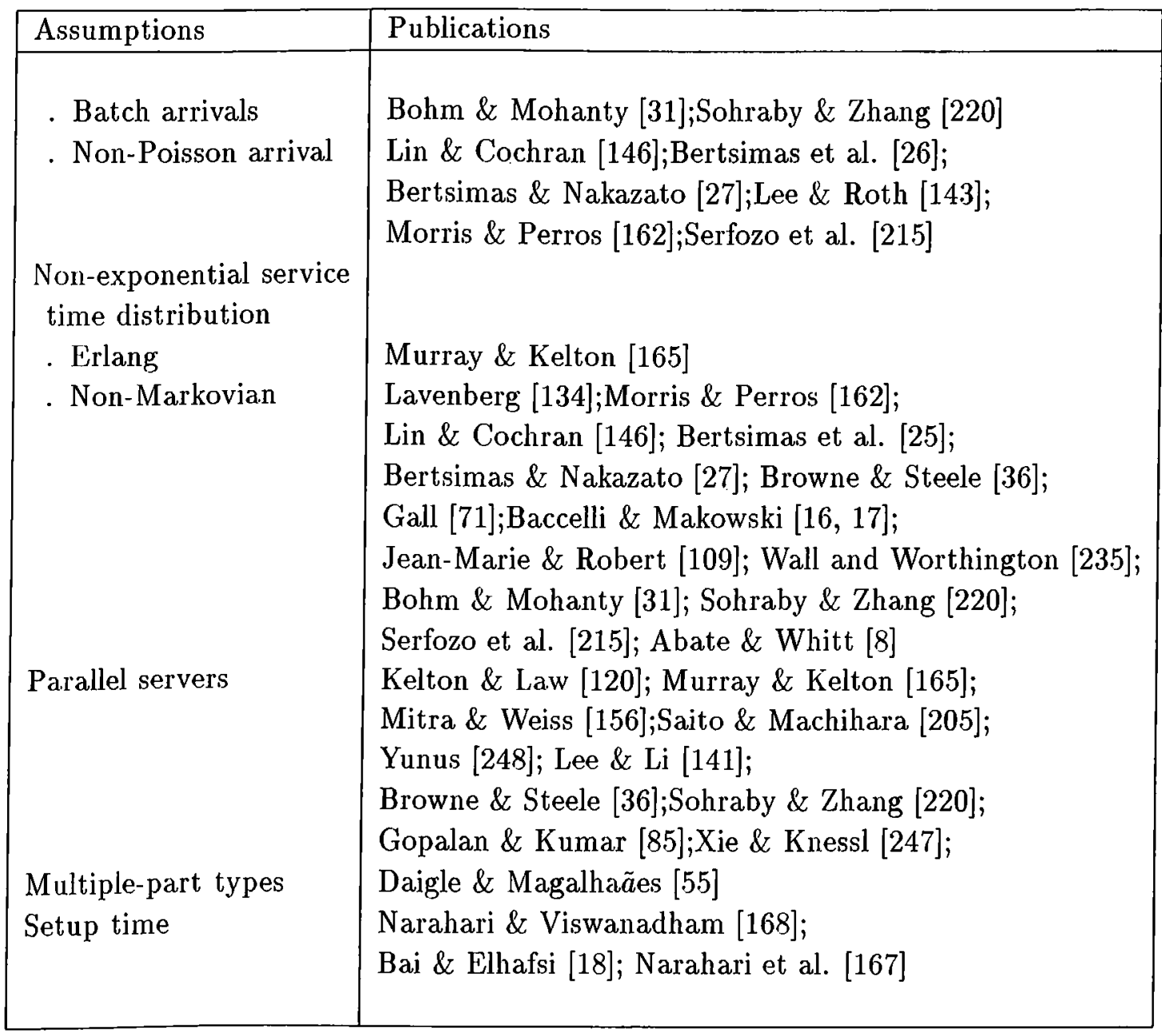

special case of generalized Erlangian distributed repair times is also presented.

Another complexity in modeling the real-life serial line production systems is the existence of machine breakdowns. While Lin and Cochran [146] make use of a simulation based approach in order to study the transient behavior of assembly line with machine breakdown, Jenq [110] and Bruneel [41] follow an analytical approach to analyze the transient behavior of a buffer system. Jenq [110] develops an approximate algorithm to calculate the mean and the variance of the queue length for a discrete-time buffer system with service interruptions and Bruneel [41] presents a new analytical technique for the derivation of the exact time-dependent queueing behavior of exactly the same model as in Jenq [110]. The comparison reveals that the results of Jenq's approximate algorithm and Bruneel's exact algorithm are quite close to each other. However, their 
analyses are restricted to infinite-capacity queues, and these algorithms will be of a more complex nature as more complicated state descriptions and more general types of server interruption processes (Bruneel $[37,38,39,40]$ ) are involved in such systems.

The arrival process is typically assumed to be Poisson because the exponential distribution is the only continuous distribution with the memoryless property. There are only three papers distinguishing the Poisson arrival type in the literature: Lee and $\mathrm{Li}$ [141] analyze the transient behavior of Markovmodulated Poisson arrival queues with multiple exponential servers. The queue is modeled in discrete-time domain with its time index characterized by packet arrivals. They first derive expressions for the one-step transition probabilities of the queue. Taking advantage of the matrix geometric solution for the onestep transition probability matrices, they simplify the evaluation of transient queue length distributions. Compared with the conventional transient analysis, their method can analyze a queue with overload control and finite buffer size. Lee and $\mathrm{Li}$ [142] also present a methodology to analyze the transient behavior of a queue-length-dependent switched Poisson arrival queue. The queue has infinite buffer capacity with an exponential server with its rate driven by a twostate Markov chain. It can be considered as a special quasi-birth-death (QBD) process whose transient behavior is analyzed in detail by Zhang and Coyle [249]. However, their result does not lead directly to transient analysis of such systems under overload control. Lee and $\mathrm{Li}$ [142] take a different approach from Zhang and Coyle [249]. A discrete-time technique is used to attack the problem where the time is indexed at packet arrivals. They first derive expressions for the one-step transition probabilities. The probability distributions of queue length at any arrival epoch can be iteratively calculated using the Kolmogorov forward equation. Taking advantage of the geometric form of the transition probabilities, they further simplify the evaluation of the forward equation. Actually, a complete algorithm for evaluation of transient queue can be found in Lee [144]. They also explore the queue transient performance as affected by the mean sojourn times of the underlying two-state Markov chain for the arrival process. Finally, Bohm and Mohanty [35] consider a Markovian queueing 
system in discrete time in which parts arrive from different sources in batches of various size. Using a lattice path representation in higher dimensional space they derive the time-dependent joint distribution of the number of arrivals of various types and the number of completed services. The distribution for the corresponding continuous time model is found by using weak convergence.

The typical queue discipline assumed in the serial line literature is the firstcome-first-served (FCFS) discipline. However, Jean-Marie and Robert [109] analyze the transient behavior of the single server queue under the processor sharing discipline. Under fairly general assumptions, they give the rate of growth of the number of customers in the queue as well as the asymptotic behavior of the residual service times described in terms of renormalized point process.

In the general context, a multiple-part type serial line production system is a n-machine m-part-type serial production line. The system consists of $\mathrm{n}$ machines and $(\mathrm{n}-1) \mathrm{m}$ buffers. Type $\mathrm{i}$ parts travel in a fixed sequence: Buffer $(0, i)$, Machine 1, Buffer $(1, i)$, Machine 2, Buffer $(2, i), \ldots .$, Buffer $(n-1, i)$, Machine $n, \operatorname{Buffer}(n, i)$. Buffers are assumed homogeneous. That is, $\operatorname{Buffer}(j, i)$ holds only identical, interchangeable parts: Type $i$ parts that have completed operations on Machine $j$ and are waiting for an operation on Machine $j+1$. This brief explanation about the configuration of the multiple-part type serial line production systems indicates that such a system is difficult to analyze. Therefore, very few authors have attempted to study such systems. Among these, Daigle and Magalhães [55] obtain the time-dependent probabilities for the joint distribution of the number of arrivals and departures in $[0, t]$ for the $M / M^{i j} / 1$ queue. This queue has the exponential service with parameters $\mu_{i j}$, depending on the types of the successive parts attended. Their results, which are presented in the matrix form, have a simple intuitive interpretation. These results complement those of Magalhães and Disney [149] which address the characteristics of the stationary departure process of this system. In another. study, Bai and Elhafsi [18] study a deterministic one-machine two-product manufacturing system with setup changes. They formulate the problem as an optimal control. The transient solution is obtained by partitioning the 
surplus/backlog space into two mutually exclusive major regions. In one region the optimal solution is obtained by inspection. In the second region an algorithm that gives the optimal state trajectory is developed.

The setup time or switch-over time is assumed to be negligible because most of the relevant studies in the literature analyze the production systems with single-class of items. There are only few studies in the literature filling this gap to an extent, for example Narahari and Viswanadham [168], Bai and Elhafsi [18], and Narahari, Hemachondra \& Gaur [167] analyze the transient state of a production system with setup changes: Bai and Elhafsi [18] consider a deterministic one-machine two-item production system. They formulate the problem as an optimal control and divide the planning horizon into a transient period and a steady-state period. For the transient period, an algorithm involving a line search procedure is developed and a feedback control policy is found to be the optimal solution. Narahari and Viswanadham [168] and Narahari, Hemachondra \& Gaur [167] focus on transient analysis of Markovian models of production systems. Narahari and Viswanadham [168] discuss two problems for demonstrating the importance of transient analysis: the first problem is concerned with the computation of distribution of time to absorption in Markov models of production systems with deadlocks or failures, and the second problem shows the relevance of transient analysis to a multi-class production system with significant setup times. Such a facility that involves the production of several classes of items belonging to different priority levels and involving significant setup times is studied in detail by Narahari, Hemachondra \& Gaur [167]. The numerical experiments of the two studies confirm that the transient performance of the system can be significantly different from the performance predicted by the steady-state analysis and conclude that transient analysis has important implications for decision making during design and operation.

The existing studies should be extended to develop methods for the analysis of more complicated systems such as longer lines with non-exponential distributions and multiple-part types. As can be seen from Table 2.4, literature is lacking in this regard. Thus, there is the opportunity for developing 
approaches that address the complexity of serial line production systems by combining the existing approaches or otherwise.

\section{Methodology}

Based on the methodology followed, the transient analysis literature of the serial line production systems can be classified in the following ways:

(1) Analytical approach (exact vs approximate analysis).

(2) Heuristics oriented approach.

(3) Simulation based approach.

The classification of the literature based on the methodology followed is presented in Table 2.5.

There is some cross fertilization among these approaches. For example, some analytical approaches use simulation to generate or evaluate alternative initial states. In the following discussion, the approaches are classified on the basis of their main emphasis.

\section{Analytical approach}

If the relationships that compose the models are simple enough, it is possible to use mathematical methods (such as algebra, calculus, or probability theory) to obtain exact information on questions of interest. However, only special systems have exact solutions due to the complexities introduced by the buffers in the serial line production systems. In such cases, the use of approximate methods is the only alternative.

\section{Exact analysis}

In this section, we describe serial line production system models that have exact analytical solutions. Such models are important because (1) exact 
Table 2.5: Classification from the methodology viewpoint

\begin{tabular}{|l|l|}
\hline Methodology & Publications \\
\hline Analytical methods & \\
Exact analysis & Lavenberg [134];Odoni \& Roth [172]; \\
& Kelton \& Law [120];Abate \& Whitt [1, 2, 3]; \\
& Parthasarathy [182];Mitra \& Weiss [156]; \\
& Murray \& Kelton [156];Bruneel [41]; \\
& Abate \& Whitt [7, 5, 4, 6]; Yunus [248]; \\
& Parthasarathy \& Sharafali [183];Gall [71]; \\
& Baccelli \& Makowski [16, 17]; \\
& Daigle \& Magalhâes [55]; \\
& Bertsimas et al. [25, 26];Abate \& Whitt [8]; \\
& Klutke \& Seiford [126];Whitt [242]; \\
& Gopalan \& Kumar [84];Tan \& Kness1 [229]; \\
& Karpelevitch \& Kreinin [114]; \\
& Conolly \& Langaris [49];Gopalan \& Kumar [85]; \\
& Narahari \& Viswanadham [168]; \\
& Narahari et al. [167] \\
& Jenq [110];Massey [152];Whitt [242]; \\
& Saito \& Machihara [205];Raatikainen [190]; \\
& Csenki [54];Bertsimas \& Nakazato [27]; \\
& Asmussen et al. [15];Lee \& Li [141, 142]; \\
& Morris \& Perros [162];Bai \& Elhafsi [18]; \\
& Xie \& Knessl [247, 246];Browne \& Steele [36]; \\
& Jean-Marie \& Robert [109];Glynn [76]; \\
& Wall \& Worthington [235];Jacobson [107] \\
& Serfozo et al. [215];Sohraby \& Zhang [220]; \\
& Bohm \& Mohanty [31];Guillemin et al. [90]; \\
& Rossetti \& Delaney [197];Gallagher et al. [72]; \\
& \\
\hline
\end{tabular}

solutions are better than simulations or approximations when the models fit real systems closely, (2) they provide useful qualitative insight into the behavior of systems, and (3) the fact that they can be solved rapidly makes them essential parts of the decomposition and aggregation methods.

Most of the results pertaining to the exact analysis of serial line models are based on the derivation of the joint distributions of the performance 
Table 2.5: Classification from the methodology viewpoint (cont'd)

\begin{tabular}{|l|l|}
\hline Methodology & Publications \\
\hline \multirow{3}{*}{ Heuristics } & Roth [198];Lee \& Roth [143];Odoni \& Roth [172]; \\
Simulation based & $\begin{array}{l}\text { Roth \& Josephy [201];Roth [199];Robinson [195] } \\
\text { Lin \& Cochran [146];Deler \& Sabuncuoğlu [58]; } \\
\text { Glynn \& Heidelberger [79, 78] }\end{array}$ \\
\hline
\end{tabular}

criteria of interest via the use of transition probabilities. In order to be able to describe the behavior of a serial line production system by a Markov process, the distributions have to be of special form: exponential or, more generally, continuous phase-type distributions in the case of continuous time models; geometric or, more generally, discrete phase-type distributions in case of discrete time models. In particular, a Markov model can also be generated using higher level models such as stochastic Petri nets and discrete event simulation (Viswanadham and Narahari [234]). However, there are some exceptions for which the analysis is not based on Markov models. These exceptions are most often encountered in serial lines with no intermediate storage.

The Markov process is often described by a set of difference-differential equations. Odoni and Roth [172] estimate the expected number of customers in queue at an arbitrary instant by numerically solving the sets of simultaneous, first-order differential equations in order to examine the transient behavior of infinite-capacity, single-server, Markovian queueing systems. Abate and Whitt [8] also use the differential equations in order to describe the time-dependent moments of the workload process in the $M / G / 1$ queue. The $M / M / 1$ workload process is also discussed in Section 6 of Abate and Whitt [7].

Another set characterizing the transient behavior is of the integral equations. Gopalan and Dinesh Kumar [84, 85] deal with a merge production system to study the effect of the parameters on utilization and availability, busy period, and blocked period of various machines in the system, respectively. For this purpose, they develop a mathematical model using semi-regenerative phenomena and system of integral equations satisfied by various state 
probabilities are obtained. An efficient numerical method is used to solve the system of integral equations obtained. Tan and Knessl [229] derive integral representations for the time-dependent distribution functions of the $M / M / 1$ queue described by the unfinished work and also a finite-capacity version of the model where the parts who would cause the unfinished work to exceed a given capacity are rejected and lost. This yields a new set of approximations for describing the transient behavior of the $M / M / 1$ queue and they also show that their formulas yield some interesting insights into how the process settles to its steady-state distribution.

Bailey $[20,21,19]$ show that the transient behavior of the queue-length process in the $M / M / 1$ model can be described using double transforms with respect to space and time. Abate and Whitt [7] discuss how the Laplace transform analysis of Bailey $[20,21]$ can be persuaded to yield additional insights about the time-dependent behavior of the queue-length process in the $M / M / 1$ model. They establish a transform factorization that leads to a decomposition of the first moment as a function of time into two monotone components and also facilitates developing approximations for the moments and determining their asymptotic behavior in the long run. Parthasarathy [182] and Parthasarathy and Sharafali [183] also obtain the transient solution for the single-server and the many-server Poisson queue via the use of Laplace transforms. Bertsimas et al [25] formulate the problem of finding simultaneously the waiting time distribution and the busy period distribution of the $G I / G / 1$ queue with arbitrary distributions as a Hilbert problem. . Bertsimas et al [26] further obtain closed form-expressions also for the Laplace transforms of the waiting-time distribution and the busy-period distribution by formulating the problem as a two-dimensional Lindley process and then transforming it to a Hilbert factorization problem. Laplace transforms are also used in developing a series expansion that is known as the methodology of Sharma [216]. Conolly and Langaris [49] discuss a series formula of Sharma [216] for the computation of time-dependent state probabilities in $M / M / 1$ queueing systems with emphasis on methods that avoid Bessel functions. Solution methods based on complex analysis techniques for a more general 
class of continuous time quasi-birth-death (QBD) processes using Laplace inversions are also provided in Zhang and Coyle [250] and Kobayashi and Ren $[130,129,131]$. The last use of the Laplace transforms to be mentioned is that they help the development of most of the asymptotic expansions. Mitra and Weiss [156] analyze a model of blocking in teletraffic using a combination of Laplace integral asymptotics and large deviations theory.

In the literature, very few authors have analyzed the transient behavior of the serial line production systems by making use of the higher order moments of the performance measures of interest due to the difficulty involved. Gall [71] makes use of the Laplace-Stieltjes transforms to derive the probability distribution of the overall sojourn time of a serial line production system in case of a renewal arrival process and arbitrarily distributed identical successive service times. Abate and Whitt [3] show that the factorial moments of the queue length as functions of time when the $M / M / 1$ queue starts empty have interesting structure, which facilitates developing approximations. Simple exponential and hyperexponential approximations for the first two moment functions help to show how the queue approaches steady-state as time evolves. These formulas also help to determine if steady-state descriptions are reasonable when the arrival and service rates are nearly constant over some interval but the process does not start in steady-state. Abate and Whitt [8] also characterize the time-dependent moments of the workload process in terms of a differential equation involving lower moment functions and the time-dependent server-occupation probability.

Another way of analyzing the transient behavior of the queueing systems is via the use of generating functions. Baccelli and Makowski $[16,17]$ make use of an exponential martingale used by Rosenkrantz [197] in order to calculate the Laplace transform of the length of the busy period for the $M / G I / 1$ queue. It provides a unified probabilistic framework for deriving well-known generating functions for the $M / G I / 1$ queue with the help of arguments from renewal theory. Some of these generating functions are the Pollaczek-Khintchine formula, the transient generating function of the number of customers at departure epochs and the generating function of the number of customers 
served in a busy period. Karpelevitch and Kreinin [114] also treat the generating function of the joint waiting-time and queue-length distributions at the arrival epochs of Poissonian serial lines.

Most of the authors in the literature assume empty initial conditions. However, there is a study by Yunus [248] that presents an exact expression for the transient blocking probability in the $M / M / n$ loss system, assuming any initial condition. This is a generalization of Riordan's result [193] and could find application in a method to approximate loss systems with non-homogeneous Poisson arrivals. In addition, Kelton [119], Kelton and Law [120], and Murray and Kelton [165] present transient results for $M / M / n, M / E_{r} / 1, E_{r} / M / 1$, and $M / E_{r} / 2$ queues and use them as benchmarks to evaluate alternative initialization methods for simulation of similar systems.

\section{Approximate analysis}

It appears from the preceding section that exact solutions of relatively short serial lines are available for a wide range of models. However, it seems hopeless to expect to obtain exact solutions of serial lines with more machines even when more powerful computers are available. The work on the longer lines involves models that are not tractable, or are subject to numerical problems, or are too limited to be of interest. Therefore, the use of approximate solutions is the only viable alternative. The purpose of this section is to review the literature devoted to approximate methods.

Towsley [230] applies the well-known reflection principle for random walks to the analysis of transient $M / M / 1$ queueing system. A closed-form solution is obtained for the probability that exact number of arrivals and departures occur over an integral of length $t$ in an $M / M / 1$ queueing system that contains a particular number of parts at the beginning of the interval. The derivation of this probability is based on the calculation of the number of paths between two paths in a two-dimensional coordinate system. It is easier to solve for the probability of a specific sample path during the time interval for the joint distribution of the number of arrivals and departures within that specific interval. Application of the reflection principle then allows to determine the 
latter distribution.

Abate and Whitt $[1,2,3,7,5,4,6]$ describe the transient behavior of onedimensional reflected Brownian motion (RBM) associated with the $M / M / 1$ queue. Since RBM can serve as rough approximations for many queueing processes, the results help to describe how a large class of queueing processes approach steady-state. These results provide simple analytical approximations in the spirit of the empirical work by Odoni and Roth [172]. The RBM is also applied to gain additional insight into queueing simulations in Whitt [240, 239]. Asmussen [14] makes use of the Brownian approximations in order to study the heavy traffic behavior of a number of standard queueing simulation procedures like sample averaging and regenerative simulation. In another study, Louchard [147] shows that several stochastic variables associated with a finite population queueing system can also be approximated by Brownian motions using weak convergence theorems. The result of this study suggests that queue length, unfinished work, storage occupied (in a computer model), and idle time show different limiting behavior depending on the arrival and service distribution.

Guillemin, Mazumdar, and Simonian [90] show that the classical diffusion approximation by an Ornstein-Uhlenbeck process captures well the average values of the transient variables of $M / M / \infty$ queueing system while the asymptotic distributions of these variables depart from those corresponding . to the Ornstein-Uhlenbeck process. They also find out that local Brownian approximation gives the same asymptotic results along with the respective tail behavior of the limit distributions regarding the transient characteristics of the $M / M / \infty$ system.

Saito and Machihara [205] analyze the transient behavior of a Markovian loss system with heterogeneous inputs. They apply the properties of system recovery and covariance functions to the analysis of the time congestion measurement whose variance is obtained for a single machine model with multiple servers by Machihara [148] via the use of the properties of eigenvalues. Approximations are proposed using these properties. The results are significant for management and control in the integrated services digital network. 
The sample path behavior of any serial line can be described by means of recursive equations, mainly for establishing qualitative properties such as monotonicity and reversibility. The approach of Klutke and Seiford [126] uses these equations to compute the transient expected output times from serial line production systems with no waiting positions.

Bertsimas and Nakazato [27] use the method of stages combined with the separation of variables and root finding techniques together with linear and tensor algebra in order to study the transient behavior of the $M G E_{\mathrm{L}} / M G E_{\mathrm{M}} / 1$ queueing system, where $M G E$ is the class of mixed generalized Erlang distributions which can approximate any arbitrary distribution. They also make use of the Laplace transforms to find simple closed-form expressions for the queue-length distribution and the waiting-time distribution under FCFS discipline when the system is initially empty, and the busy-period distribution.

Knessl [127] analyzes the transient behavior of the classic Erlang loss model. Xie and Knessl [247] further obtain several asymptotic formulas for this model under the heavy usage conditions. In constructing these approximations, the singular perturbation techniques such as the ray method, boundary layer theory, and the method of matched asymptotic expansions are used. Xie and Knessl [246] also take a similar approach in giving asymptotic expansions for the probability that the system contains a particular number of parts at time $t$ in the classic $M / M / 1$ queue with finite/infinite capacity. Their results are based on both the asymptotic expansion of an exact integral representation for the probability of interest and applying the ray method to a scaled form of the forward Kolmogorov equation describing the time evolution of the probability. Actually, the model on which Knessl [127], Xie and Knessl [247], and Xie and Knessl [246] study is exactly the same as the one used by Mitra and Weiss [156] to analyze the transient behavior in the Erlang's model for large trunk groups and various traffic conditions. Serfozo, Szczotka, and Topolski [215] characterize the asymptotic distribution of the difference between the waiting time and the service time of any part. Their results apply, in particular, to the $G I / G / 1$ system and systems in which the inter-arrival and service times are stationary, regenerative, semi-stationary, asymptotically stationary, and their 
sums satisfy certain functional limit laws.

Browne and Steele [36] obtain the distribution of the busy period in an $M / G / \infty$ queueing system with exceptional first service by deriving the distribution of the length of a clump in a coverage process where the first line segment of a clump has a distribution that differs from the remaining segments of the clump. Their result also provides the tool necessary to analyze the transient behavior of an ordinary coverage process, namely the depletion time of the ordinary $M / G / \infty$ system.

Lee and $\mathrm{Li}[141,142]$ take an approach that is different from the conventional transform approach, a discrete-time analysis with its time indexed by packet arrivals. They derive expressions for the transition probabilities of the two dimensional Markov chain formed by the queue length and the underlying Markov chain in adjacent intervals. The probability distribution of queue size at any arrival epoch can then be calculated iteratively using the Kolmogorov forward equation. Due to the geometric solutions for the one-step transition probabilities, they are able to devise an efficient algorithm to reduce the complexity of directly evaluating the forward equation.

In solving discrete time queueing models by numerical techniques, the computational requirements (computer memory and time) are practical limitations and are particularly dependent on the number of discrete time intervals required in the discrete distribution chosen to match the general service time distribution. Wall and Worthington [235] show that the minimum number of points required for matching to the first two moments depends on the size of the discrete interval relative to the mean and also on the coefficient of variation.

Most approximate methods are based on decomposition. The common idea is to decompose the analysis of the original model into the analysis of a set of smaller subsystems which are easier to analyze.

Csenki [54] shows that a suitable probabilistic reasoning using absorbing Markov chains can be used to obtain the probability mass function and the 
cumulative distribution function of the joint distribution of the sojourn times. The key method of analysis employed in this study is based on the introduction of equivalent absorbing Markov chains whose state space is partitioned into groups of states which are visited in a predetermined order, each one, until absorption takes place. This device allows the variables of interest to be studied by using elementary techniques for absorbing Markov chains.

Morris and Perros [162] present an approximation algorithm for the analysis of a discrete-time tandem network of cut-through queues. The tandem network is analyzed by decomposing it into individual queues. Each cut-through queue is then analyzed numerically in isolation assuming that its arrival and service processes are known. Using this approach, the queue-length distribution, the packet loss, the blocking probability between queues, and the throughput rate is obtained for different traffic parameters, subpacket-to-packet ratios, and queue sizes.

The technique proposed by Bohm and Mohanty [31] presents a sample path of the queueing process by a lattice path which can be decomposed into distinct segments corresponding to periods where the server is idle or busy. These segments can be enumerated (and thus their probability distribution can be determined) by standard methods of lattice path combinatorics. The approach can be considered as a reasonable alternative to analytical methods. Actually, this point of view is by no means new. Takács [225] and Feller [61] have demonstrated very clearly the power of combinatorial methods in applications to stochastic processes. Moreover, this approach is successfully . applied to Markovian queueing systems in discrete time by Bohm [31], Bohm and Mohanty [32, 33, 35], Kanwar and Jain [111], and Kanwar, Jain, and Gupta [112].

In the transient analysis of multi-server discrete-time queues, Sohraby and Zhang [220] show that once the spectral decomposition of the probability generating matrix of the arrival process is obtained, the complete solution in the transform domain may be given. Using the complex analysis technique and Cauchy's integral formula, they present an efficient numerical method for 
the numerical calculation of a few performance measures of interest, namely transient probability of queue being empty, and the mean of the queue-length distribution. Actually, this study is the generalization of the previous work by Sohraby and Zhang [219] where the spectral decomposition is used for the transient analysis of a single server queue.

The bulk of the published literature on the transient analysis of the serial line production system takes the analytical approach. The main problem with this work is the very narrow applicability of the models: they do not consider the full complexity of general production systems, i.e., almost all of the studies assume reliable machines. Indeed, some models are so singular that the problems seem to be contrived to fit the model rather than the other way around. Another difficulty is the computational effort involved: it is hard to find models that will solve reasonably sized problems efficiently. The models are also excessively rigid. There is a need to develop models that factor in the full complexity of the serial line production systems (perhaps hierarchically), yet are efficient enough to obtain solutions in real time where needed, and are flexible enough to distinguish between soft goals and hard constraints.

\section{Heuristics oriented approach}

There are many classes of serial line production systems for which, due to the mathematical complexities, exact solutions cannot be obtained in a form useful for applications. In fact, even for simple systems few results are available to characterize transient response. However, use of heuristics has not been actively investigated in the literature.

Through an examination of numerical solutions to Markovian queueing systems, Roth [198] shows that the expected queue length eventually approaches to an equilibrium value in an approximately exponential manner. Based on this observation a heuristic is proposed for approximating the transient expected queue length for Markovian systems by scaling the numerical solution of an $M / M / 1$ system. 
Lee and Roth [143] present an approximation for the transient expected queue length (excluding the customer in service). This work is based on Lee [145] and is an extension of the empirical research conducted by Odoni and Roth [172] on the transient response of stationary Markovian queueing systems. This earlier work establishes guidelines for estimating the effective length of the transient period. Using these results, they propose a heuristic for estimating transient behavior. This work is application oriented and their objective is to provide a closed form-expression for non-specialists to use in practice.

It is important to recognize that the existing heuristics examine the accuracy of the solution techniques only for Markovian systems. Verification for truly general cases is a difficult task. At this time, for more general systems, it is not possible to obtain transient solutions which are sufficiently accurate to verify the accuracy of approximate solutions generated by the heuristic. This is certainly one area for further research.

\section{Simulation based approach}

Simulation is certainly more tractable than analytical formulations of production system problems. With simulation, there is no concern about feasibility since there is no need to make any unnecessary simplifying assumptions. The simulation model can be built as close to reality as one needs to. Therefore, simulation is proposed as a tool for the transient analysis of the serial line production systems.

Lin and Cochran [146] study the transient behavior of assembly line for the often encountered dynamic event of machine breakdown, in much detailed by computer simulation. The event of a machine breakdown is simulated by stopping the operation of one or more servers at a user-selected work station for some time period specified in the experimental design frame. Due to system and job characteristics complexity, they utilize the discrete-event simulation that provides a modeling power unavailable from analytical models. To combine the advantages of analytical and simulation methods, the simulation results of assembly line transient behavior are modeled by dynamic meta models in the form of first-order continuous exponential delay functions which are the 
solutions to continuous first-order differential equations. Estimating late part finishing due to machine breakdown is easily accomplished from the meta model and has been useful to management involved in production control.

Andradottir [12] considers the optimization of the transient behavior of a discrete event system modeled as a general state space Markov chain whose distribution depends on the decision parameters. They also show how simulation and the likelihood ratio method can be used to evaluate the performance measures of interest and its gradient, and they present conditions that guarantee the convergence of the Robbins-Monro stochastic approximation algorithm to the optimal values of the decision parameters.

The integrated research approach of using simulation in modeling system details and analytical method in describing system transient behavior is a powerful methodology for analyzing complex large-scale systems. It provides meaningful results that cannot be achieved by either analytical or simulation methods alone. There is much scope for developing and evaluating simulation based techniques for other operational problems specific to serial line production systems.

The limitations of the existing models can be stated as follows:

- Almost all the models consider the systems of very limited size. Kelton and Law [120], Lin and Cochran [146] study one-machine systems while Gopalan and Dinesh Kumar [84], Klutke and Seiford [126] consider twomachine systems.

- The assumptions of the corresponding studies are so limiting that they do not reflect the common nature of the transfer lines of practical importance. 


\subsection{Summary}

Works on the transient behavior of the output process of transfer lines are scarce in the literature. And also, there are little implications about the solution techniques. However, more importantly, there is no work that exactly matches with our models that will be described and solved in the succeeding chapters. 


\section{Chapter 3}

\section{On the Distribution of Throughput}

\subsection{Introduction}

As seen from the previous chapter, most of the works in the literature deals with the calculation of the first order moment of the steady-state performance measures. However, it is easily seen that there is a lack in the issues of the transient analysis and the second order moment of the performance measures even in Markovian systems, where all the distributions are exponential or phase-type. In this chapter, we propose an analytical method for estimating the performance measures of a serial production line with reliable machines and finite buffers.

\subsubsection{The model assumptions and notation}

Before going into details, it will be beneficial to state the major assumptions. The assumptions listed in Table 3.1 describe the mostly encountered production line in the literature (Dallery and Gershwin [56]). 
Table 3.1: The assumptions

1. The production line is a serial arrangement of a finite number of $n$ machines. Each machine can operate on one unit of product at a time and has internal storage capacity for that unit.

2. The arrival process is assumed to be Poisson. Therefore, the inter-arrival times for part $i, T_{\lambda}^{i}(i=1, \ldots)$, are independent identically distributed exponential random variables with density function $f_{i}(t)=\lambda e^{-\lambda t}, \lambda>0$.

3. The machines $M_{j}(j=1, \ldots, n)$ have mutually independent processing times that are also exponentially distributed with density function $f_{i}(t)=\mu_{j} e^{-\mu_{j} t}$, $\mu_{j}>0$.

4. The first buffer of the line is assumed to have zero capacity (i.e., the parts arriving the system while the first machine is busy are lost) and the last buffer is considered to have infinite capacity (i.e., last machine never gets blocked).

5. The buffers between the machines of the line have finite storage capacities. There are no overflows or lost parts. If a machine has finished working on a part and the next downstream buffer is full, that machine becomes blocked and stops processing parts until a buffer slot becomes available (blockingafter-service policy).

6. All machines are reliable and produce no bad parts.

7. No batching and no setup times (single product) are considered.

8. The output process is not necessarily stationary (i.e., a steady-state distribution for the output of the system under consideration may not exist).

9. The production line assumes idle and empty initial conditions.

Since the same notation is used throughout the thesis, it would be beneficial to give the common notation at the very beginning (Table 3.2).

\subsubsection{Organization}

In this chapter, we propose an analytical method for estimating the performance measures of a serial production line with reliable machines and finite buffers. First section is devoted to the model development. In this section, the evolutions of the stochastic processes of the random variable, $N_{1}(t)$ for the atomic model, $N_{2}(t)$ for the two-machine-one-buffer system, and $N_{3}(t)$ for the three-machine-two-buffer system, respectively, are determined. And 
Table 3.2: The notation

$N_{j}(t)$ : random variable of the number of parts that have left machine $j$ up to time $t$ in an $n$-machine transfer line, $j=1, \ldots, n$

$l$ : number of parts leaving the system at an instance in time

$k:$ index of the period in which the system is

$p^{c, k}:$ number of parts arriving the system under Case $c$ in period $k$

$n$ : number of machines in the system

$\lambda$ : arrival rate to the system

$\mu_{j}:$ service rate of machine $j, j=1, \ldots, n$

$T_{\lambda}^{i}: \quad$ inter-arrival time for part $i$

$T_{\mu_{j}}^{i}$ : $\quad$ service time for part $i$ on machine $j, j=1, \ldots, n$

$b_{m}$ : size of the $m^{\text {th }}$ buffer in the $n$-machine transfer line, $m=0,1, \ldots, n$

$b_{i}^{m}$ : size of the $m^{\text {th }}$ buffer in the $n$-machine transfer line at the instant the $i^{\text {th }}$ part leaves the system of interest, $m=0,1, \ldots, n$

$f_{\lambda, \mu_{j}}(t)$ : probability density function with parameters $\lambda$ and $\mu_{j}$

$F_{\lambda, \mu_{j}}(t):$ cumulative distribution function with parameters $\lambda$ and $\mu_{j}$

$f_{\lambda, \mu_{j}}^{l}(t): l^{\text {th }}$ convolution of the density function $f_{\lambda, \mu_{j}}(t)$

$F_{\lambda, \mu}^{l}(t): \quad l^{t h}$ convolution of the distribution function $F_{\lambda, \mu_{j}}(t)$

$E\left(N_{n}(t)\right)$ : mean of the throughput, the number of parts leaving the $n$-machine system at time $t$

$V\left(N_{n}(t)\right)$ : variance of the throughput, the number of parts leaving the $n$-machine system at time $t$

$E(t)$ : mean of the throughput rate at time $t$

$V(t):$ variance of the throughput rate at time $t$

$C V(t)$ : coefficient of variation of throughput rate at time $t$.

$E$ : steady-state mean of the throughput rate

$V:$ steady-state variance of the throughput rate

$C V:$ steady-state coefficient of variation of throughput rate

$E_{d}$ : desired throughput rate

also, how the corresponding characterizations of the distribution of the output process of the systems under consideration can be readily utilized to obtain the various moments of throughput, is explained in this section. The final section consists of an experimental design that helps to determine whether the model can capture the true transient and steady-state behaviors of the relevant systems. 


\subsection{The model development}

\subsubsection{Modeling}

As stated in the previous section, the general system under consideration is an $n$-machine- $(n+1)$-buffer line. However, we specialize on three systems : The atomic model, two-machine-one-buffer system, and three-machine-twobuffer system. This section gives the verbal and graphical descriptions of the transient behavior of these systems. The corresponding descriptions help us to write down the representations for the number of parts leaving the system at arbitrary instants in time. Then, the relevant representations are utilized to obtain the distribution of throughput by which the mean and variance of the number of parts leaving the system are calculated. This approach is explained in detail after presentation of the stochastic process evolutions of the related systems.

The atomic model

The atomic model corresponds to one-machine system as depicted in Figure 3.1:

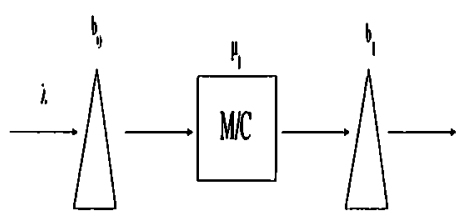

Figure 3.1: $T_{\lambda}^{i} \sim \exp (\lambda), T_{\mu_{1}}^{i} \sim \exp \left(\mu_{1}\right) \forall i ; b_{0}=0 \& b_{1}=\infty$

The evolution of the stochastic process of the random variable, $N_{1}(t)$, is presented in Figure 3.2.

The memorylessness property of the inter-arrival times (i.e., the Poisson arrival process) and the zero capacity of the first buffer in the line facilitate the representation of the stochastic process evolution for the atomic model. Whenever a part leaves the system, say part $(i-1)$, the next one arrives $T_{\lambda}^{i}$ time units later. Hence, the number of parts leaving the system at time $t$ can 


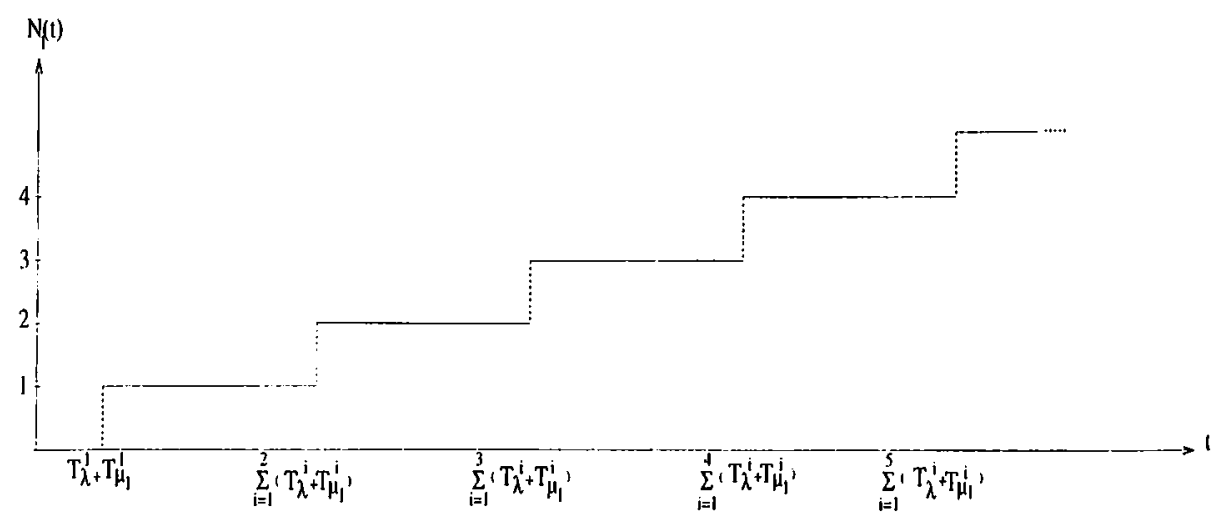

Figure 3.2: Evolution of the stochastic process, $N_{1}(t)$.

be written as

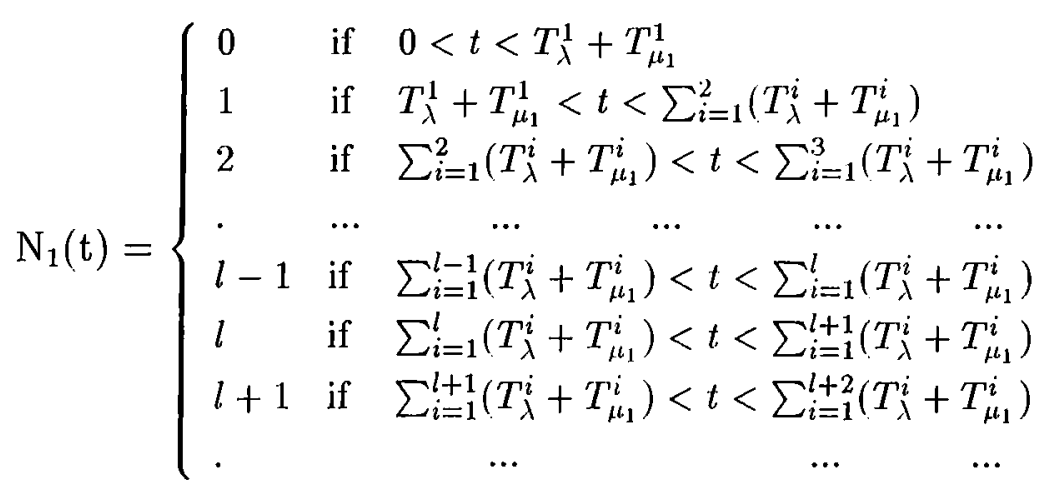

\section{Two-machine-one-buffer system}

The system under consideration is illustrated in Figure 3.3:

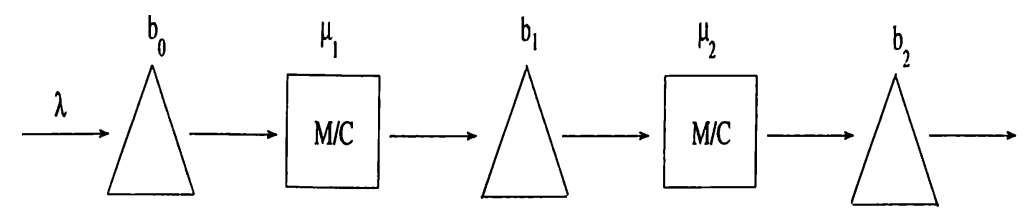

Figure 3.3: $T_{\lambda}^{i} \sim \exp (\lambda), T_{\mu_{j}}^{i} \sim \exp \left(\mu_{j}\right) \forall i, j=1,2 ; b_{0}=0, b_{1} \geq 0, \& b_{2}=\infty$.

Now, there occurs an increase in the number of the sources of variability $\left(T_{\lambda}^{i}, T_{\mu_{1}}^{i}\right.$, and $\left.T_{\mu_{2}}^{i}, i \geq 1\right)$ due to the more number of machines and buffers in the system of interest and this leads to the existence of two mutually exclusive and collectively exhaustive events that describe the behavior of the system: $T_{\lambda}^{i}+T_{\mu_{1}}^{i}>T_{\mu_{2}}^{i-1}$ and $T_{\lambda}^{i}+T_{\mu_{1}}^{i} \leq T_{\mu_{2}}^{i-1}$ for each part, $i$. In the first case, 
no part accumulates in the buffer. In the second one, the first machine gets blocked some time depending on the relative magnitudes of the inter-arrival times and the service times of the machines, and also the capacity of the second buffer of the line, $b_{1}$. In order to capture the true behavior of the system, we consider an aggregate case in which the first condition, $T_{\lambda}^{i}+T_{\mu_{1}}^{i}>T_{\mu_{2}}^{i-1}$, is let be valid for $p^{1, k}$ parts and the second condition, $T_{\lambda}^{i}+T_{\mu_{1}}^{i} \leq T_{\mu_{2}}^{i-1}$, is let be valid for $p^{2, k}$ parts in period $k$. By the way, a period is defined as the time frame in which mutually exclusive and collectively exhaustive descriptions of the system behavior appear. However, there may be time frames in which the conditions for particular cases may not hold. That is, during the evolution of the stochastic process, $N_{2}(t)$, which is defined as the number of parts leaving the two-machine transfer line under the aggregate case, time frames in which $p^{1, k} / p^{2, k}$ is equal to 0 in period $k$ can be observed. This means that all the parts leaving the first machine find the second machine busy/starved in period $k$. Appendix A.1 clarifies the advantage of making the definition of " period " in terms of obtaining the corresponding analytical derivations in a simpler way. The $p^{1, k}$ and $p^{2, k}$ variables are considered to be known in advance by the production manager and, accordingly, the analytical derivations for the number of parts leaving the system, $N_{2}(t)$, can be modified at any instance in time. The evolution of the stochastic processes and the corresponding analytical derivations for $N_{2}(t)$ under Case 1, Case 2, and the aggregate case, in which the first case holds for $p^{1, k}$ parts and the second case for $p^{2, k}$ parts in period $k$, for the two-machine transfer line with finite buffer storage, are given in the Appendix A.1.

\section{Three-machine-two-buffer system}

The system under consideration is illustrated in Figure 3.4:

In this system, there arises four mutually exclusive and collectively exhaustive events that describe the behavior of the line: $T_{\lambda}^{i}+T_{\mu_{1}}^{i} \geq T_{\mu_{2}}^{i-1}$ and $T_{\lambda}^{i}+T_{\mu_{1}}^{i}+T_{\mu_{2}}^{i} \geq T_{\mu_{2}}^{i-1}+T_{\mu_{3}}^{i-1}, T_{\lambda}^{i}+T_{\mu_{1}}^{i} \geq T_{\mu_{2}}^{i-1}$ and $T_{\lambda}^{i}+T_{\mu_{1}}^{i}+T_{\mu_{2}}^{i}<T_{\mu_{2}}^{i-1}+T_{\mu_{3}}^{i-1}$ $T_{\lambda}^{i}+T_{\mu_{1}}^{i}<T_{\mu_{2}}^{i-1}$ and $T_{\mu_{3}}^{i-2} \geq T_{\mu_{2}}^{i-1}$, and $T_{\lambda}^{i}+T_{\mu_{1}}^{i}<T_{\mu_{2}}^{i-1}$ and $T_{\mu_{3}}^{i-2}<T_{\mu_{2}}^{i-1}$. In the first condition, there is hardly ever an accumulation in the buffers. However, 


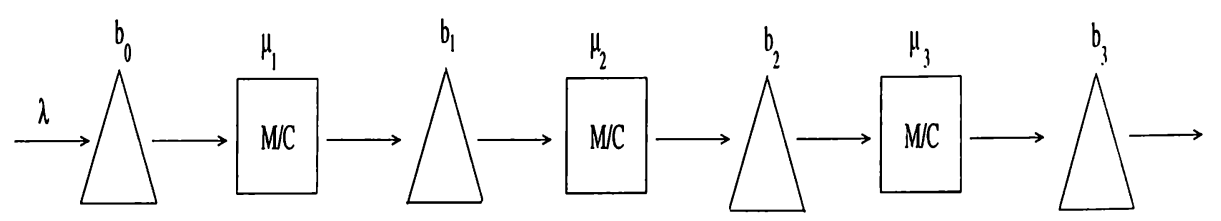

Figure 3.4: $T_{\lambda}^{i} \sim \exp (\lambda), T_{\mu_{j}}^{i} \sim \exp \left(\mu_{j}\right) \forall i, j=1,2,3 ; b_{0}=0, b_{1} \geq 0, b_{2} \geq 0, \&$ $b_{3}=\infty$.

significant changes take place in the buffer levels of the system in the second, third, and fourth conditions. Therefore, none of these cases uniquely represent the true system behavior by itself. Similarly, each condition is allowed to be valid for particular number of parts in each period under the aggregate case, which are assumed to be known in advance.

The corresponding analytical derivation for $N_{3}(t)$ under Case 1, Case 2, Case 3, Case 4, and the aggregate case, in which Case $c$ holds for $p^{c, k}$ parts in period $k, c=1,2,3,4$, for three-machine transfer line with finite buffer storages, are also given in the Appendix A.2.

\subsubsection{Approach}

In this section, we explain how the analytical derivations for the number of parts leaving the system at an instance in time can be readily utilized to obtain the various moments of the throughput. This is shown on the atomic model due to the simplicity in deriving the first and second order moments of the throughput rate of the system.

The number of parts leaving the system, the atomic model, at time $t$ has been obtained as (1) on page 50. It is deduced from this statement that,

$$
P\left(0<t<T_{\lambda}^{1}+T_{\mu_{1}}^{1}\right)=P\left(N_{1}(t)=0\right)
$$

and

$$
P\left(\sum_{i=1}^{l} T_{\lambda}^{i}+T_{\mu_{1}}^{i}<t<\sum_{i=1}^{l+1} T_{\lambda}^{i}+T_{\mu_{1}}^{i}\right)=P\left(N_{1}(t)=l\right), \quad l=1,2, \ldots
$$


By definition of the expectation,

$$
E\left(N_{1}(t)\right)=\sum_{l=1}^{\infty} l \times P\left(\sum_{i=1}^{l} T_{\lambda}^{i}+T_{\mu_{1}}^{i}<t<\sum_{i=1}^{l+1} T_{\lambda}^{i}+T_{\mu_{1}}^{i}\right) .
$$

Simultaneously, $P\left(N_{1}(t)=l\right), l=1,2, \ldots . \quad$ can be written as the difference of the convolution of distribution functions,

$$
P\left(N_{1}(t)=l\right)=F_{\lambda, \mu_{1}}^{l}(t)-F_{\lambda, \mu_{1}}^{l+1}(t) .
$$

If this is substituted into the expression (4), then we determine the mean and the variance of the number of parts leaving the system at time $t$,

$$
\begin{gathered}
E\left[N_{1}(t)\right]=\sum_{l=1}^{\infty} l \times\left(F_{\lambda, \mu_{1}}^{l}(t)-F_{\lambda, \mu_{1}}^{l+1}(t)\right), \\
E\left[N_{1}(t) \times\left(N_{1}(t)-1\right)\right]=\sum_{l=2}^{\infty} l \times(l-1) \times\left(F_{\lambda, \mu_{1}}^{l}(t)-F_{\lambda, \mu_{1}}^{l+1}(t)\right),
\end{gathered}
$$

and

$$
V\left[N_{1}(t)\right]=E\left[N_{1}(t) \times\left(N_{1}(t)-1\right)\right]+E\left[N_{1}(t)\right]-E\left[N_{1}(t)\right]^{2} .
$$

Then, the mean and the variance of the throughput rate over a length of time $t$, can be written as,

$$
E(t)=E\left[\frac{N_{1}(t)}{t}\right]=\frac{E\left[N_{1}(t)\right]}{t}
$$

and

$$
V(t)=V\left[\frac{N_{1}(t)}{t}\right]=\frac{V\left[N_{1}(t)\right]}{t^{2}} .
$$

The mean and the variance of the throughput rate in the steady-state can also be calculated as

$$
\lim _{t \rightarrow \infty} E(t)=E
$$

and

$$
\lim _{t \rightarrow \infty} V(t)=V .
$$

The major difficulty is due to the development of the closed-form expressions for the mean and the variance of $N_{1}(t)$, which are expressed as in (6) and 
(8). Therefore, the essential step turns out to be the determination of $l$ fold convolution of distribution function, $F_{\lambda, \mu_{1}}^{l}(t)$. In fact, this is the $l$ fold convolution of the random variable $T_{\lambda}^{i}+T_{\mu_{1}}^{i}$, which is hypoexponentially distributed with parameters $\lambda$ and $\mu_{1}$, respectively, in case $\lambda \neq \mu_{1}$. Otherwise, the $l$-fold convolution of the random variable $T_{\lambda}^{i}+T_{\mu_{1}}^{i}$ has an Erlang-type distribution. In other words, $T_{\lambda}^{i}+T_{\mu_{1}}^{i} \sim \operatorname{hypo}\left(\lambda, \mu_{1}\right)$ if $\lambda \neq \mu_{1}$ and $T_{\lambda}^{i}+T_{\mu_{1}}^{i} \sim$ Erlang $\left(\mu_{1}\right)$ if $\lambda=\mu_{1}$. In case the arrival rate is equal to the service rate of the server, $\lambda=\mu_{1}$, the closed-form expressions for the probability density function and the distribution function can be readily obtained by the definition of the Erlang distribution function (Rohatgi [196]). However, although the sum of hypoexponentially distributed random variables is also hypoexponentially distributed with the requirement that all the random variables are independent with different parameters (Trivedi [231]), there is no concrete information about whether the sum of hypoexponentially distributed random variables is also hypoexponential in case the parameters of the random variables are identical. At this point, we recommend to make use of the convolution method by which the closed-form expression of $F_{\lambda, \mu_{1}}^{l}(t)$ can be obtained (Rohatgi [196]):

Theorem 1. Let $(X, Y)$ be a random variable of the continuous type with probability density function $f$. Let $Z=X+Y$. Then the probability density function of $Z$ is given by

$$
f_{z}(z)=\int_{-\infty}^{z} f(x, z-x) \mathrm{dx} .
$$

Corollary 1. If $X$ and $Y$ are independent with probability density functions $f_{1}$ and $f_{2}$, respectively, then

$$
f_{z}(z)=\int_{-\infty}^{z} f_{1}(x) \times f_{2}(z-x) \mathrm{dx} .
$$

In this way, the closed-form expressions for the probability density function and the distribution function are obtained as in case $\lambda \neq \mu_{1}$ :

$$
f_{\lambda, \mu_{1}}^{l}(t)= \begin{cases}-\frac{\lambda \times \mu_{1} \times\left(e^{-t \mu_{1}}-e^{-t \lambda}\right)}{\mu_{1}-\lambda} & \text { if } l=0 \\ \frac{\lambda^{l} \times \mu_{1}^{l} \times e^{-t \mu_{1}} \times \sum_{i=1}^{l}(-1)^{i+1} \times A_{i} \times t^{l-i} \times\left(\lambda-\mu_{1}\right)^{l-i}}{(l-1) !\left(\lambda-\mu_{1}\right)^{2 l-1}} & \\ +\frac{\lambda^{l} \times \mu_{1}^{l} \times e^{-t \lambda} \times \sum_{i=1}^{l}(-1)^{i+1} \times A_{i} \times t^{l-i} \times\left(\mu_{1}-\lambda\right)^{l-i}}{(l-1) ! \times\left(\mu_{1}-\lambda\right)^{2 l-1}} & \text { if } l \geq 1\end{cases}
$$


and

$$
F_{\lambda, \mu_{1}}^{l}(t)= \begin{cases}\frac{\lambda \times e^{-t \mu_{1}}}{\mu_{1}-\lambda}+\frac{\mu_{1} \times e^{-t \lambda}}{\lambda-\mu_{1}}+1 & \text { if } l=0 \\ \frac{\lambda^{l} \times \mu_{1}^{l} \times \sum_{i=1}^{l}(-1)^{i+1} \times A_{i} \times g_{l-i}(t) \times\left(\lambda-\mu_{1}\right)^{l-i}}{(l-1) ! \times\left(\lambda-\mu_{1}\right)^{2 l-1}} & \\ +\frac{\lambda^{l} \times \mu_{1}^{l} \times \sum_{i=1}^{l}(-1)^{i+1} \times A_{i} \times h_{l-i}(t) \times\left(\mu_{1}-\lambda\right)^{l-i}}{(l-1) ! \times\left(\mu_{1}-\lambda\right)^{2 l-1}} & \text { if } l \geq 1\end{cases}
$$

where $g_{l-i}(t)$ and $h_{l-i}(t)$ are incomplete gamma functions that can be defined as $g_{l-i}(t)=\int_{0}^{t} x^{l-i} \times e^{-x \mu_{1}} \mathrm{dx}$ and $h_{l-i}(t)=\int_{0}^{t} x^{l-i} \times e^{-x \lambda} \mathrm{dx}$, and also the coefficients $A_{i}$ are numerically calculated in the Maple $\mathrm{V}$ environment by convoluting the relevant density functions. Simultaneously, these expressions are checked whether they are probability density functions and the distribution functions by the application of the following two and three-step tests, respectively in the Maple $\mathrm{V}$ environment:

- $f_{\lambda, \mu}^{l}(t) \geq 0$ for all $t \geq 0$

- $\int_{0}^{\infty} f_{\lambda, \mu}^{l}(x) \mathrm{dx}=1$

and

- $F_{\lambda, \mu}^{l}(t)$ is nondecreasing,

- $F_{\lambda, \mu}^{l}(t)$ is right continuous,

- $\lim _{t \rightarrow 0} F_{\lambda, \mu}^{l}(t)=0$ and $\lim _{t \rightarrow \infty} F_{\lambda, \mu}^{l}(t)=1$.

The substitution of expression (14) in (6) and (7) leads to the determination of exact values of the mean and the variance of number of parts leaving the system at time $t$. Finally, the mean and the variance of the throughput rate as a function of time $t$ and the steady-state mean and variance of the throughput rate by letting time, $\mathrm{t}$, approach infinity, are numerically obtained. 


\subsection{Numerical Results}

In the previous section, analytical results corresponding to the evolution of the stochastic processes under consideration are given. In this section, the numerical results concerning the output process of transfer lines are divided into two main parts: Computational efforts required and accuracy of the approximation in the estimation of the performance measures, which are the mean and variance of throughput rate.

The organization of this section is as follows: next section explains the coding environment of the proposed method. Succeeding section is devoted to the numerical results concerning the approximation accuracy. Moreover, an experiment is designed to determine whether the models can capture the true transient and steady-state behaviors of the corresponding systems.

\subsubsection{Computer Codes}

As explained in detail in the previous section, the expressions for $P\left(N_{n}(t)=\right.$ $l), n=1,2,3$ and $l=0,1,2, \ldots$ are deduced from the analytical derivations obtained via the evolution of the corresponding stochastic processes. And also, it is mentioned that substitution of $P\left(N_{n}(t)=l\right), n=1,2,3$ and $l=0,1,2, \ldots$ into $E\left[N_{n}(t)\right]=\sum_{l=1}^{\infty} l \times P\left(N_{n}(t)=l\right)$ enables us to obtain the analytical representation of the mean of the number of parts leaving the $n$-machine transfer line. The numerical results are obtained by developing the necessary codes in the Maple $\mathrm{V}$ environment and the relevant coefficients are also numerically calculated by convoluting the related density functions. Furthermore, $V\left[N_{n}(t)\right]$, variance of the number of units leaving the $n$-machine transfer line, can be obtained both analytically and numerically by substituting the relevant expressions into $E\left[N_{n}(t) \times\left(N_{n}(t)-1\right)\right]+E\left[N_{n}(t)\right]-E\left[N_{n}(t)\right]^{2}$.

The hardest problem to be dealt with at the point of deriving a closed-form expression for the particular probability density and cumulative distribution functions is the determination of the coefficients $A_{i}, i \geq 1$. The calculation 
of the corresponding coefficients via the use of the codes developed in the Maple V environment turns out to be the second step. The third one is the replacement of the coefficients in the probability density and distribution functions. Respectively, both the transient-state and steady-state probabilities are calculated. Finally, the performance measures, that are the mean and variance of throughput rate, are calculated by also using the Maple V codes. In summary:

Algorithm.

1. Analytical derivation of the closed-form expressions of the output process based on the evolution of the corresponding stochastic processes.

2. Determination of the coefficients in the closed-form expression of the distribution function via the convolution method (MAPLE V).

3. Check whether the probability density and distribution functions conform to the corresponding tests in case the coefficients, which are determined in Step 2, are substituted into the closed-form expressions of the relevant functions (MAPLE V).

4. Calculation of the transient state probabilities (MAPLE V).

5. Calculation of the steady-state probabilities (MAPLE V).

6. Calculation of the performance measures (MAPLE V).

The next section includes the comparison between the analytical results and the state-space representation and the comparison between the analytical results and the simulation. The results corresponding to the state-space representation are obtained via the codes in the Maple environment that help to calculate the performance measures in the long run and the ones corresponding to the simulation are developed in the Siman environment to calculate the performance measures at arbitrary instants in time. 


\subsubsection{Validation}

After the development of the model, an experiment is designed in order to determine whether the model can capture the true transient and steady-state behaviors of the corresponding systems.

\section{State-space representation}

For each system, the mean and the variance of throughput rate obtained by the analytical method are compared with the ones given by the statespace representations, which are developed under exactly the same assumptions for the analytical models (Tables 3.3-3.5). The state-space representations correspond to the Markov chain models that are developed via the use of balance equations and that are used to obtain the steady-state performance measures of the systems.

\begin{tabular}{|c|c|c|c|c|}
\hline$\left(\lambda, \mu_{1}\right)$ & \multicolumn{2}{|c|}{ Analytical Method } & \multicolumn{2}{c|}{ Markov Chain } \\
\hline \hline & Mean & Variance & Mean & Variance \\
\hline$(3,3)$ & 3.000 & 9.000 & 3.000 & 9.000 \\
\hline$(1,0.5)$ & 0.499 & 0.249 & 0.500 & 0.250 \\
\hline$(0.5,1)$ & 0.499 & 0.249 & 0.500 & 0.250 \\
\hline
\end{tabular}

Table 3.3: Analytical results vs state-space representations: the atomic model

\begin{tabular}{|c|c|c|c|c|}
\hline$\left(b_{1}, \lambda, \mu_{1}, \mu_{2}\right)$ & \multicolumn{2}{|c|}{ Analytical Method } & \multicolumn{2}{c|}{ Markov Chain } \\
\hline \hline & Mean & Variance & Mean & Variance \\
\hline$(0,1,3,3)$ & 0.727 & 1.650 & 0.727 & 1.653 \\
\hline$(0,1,7,3)$ & 0.834 & 1.804 & 0.833 & 1.804 \\
\hline$(2,1,3,3)$ & 0.748 & 1.686 & 0.749 & 1.687 \\
\hline$(2,1,7,3)$ & 0.871 & 1.854 & 0.873 & 1.857 \\
\hline$(5,1,3,3)$ & 0.748 & 1.686 & 0.750 & 1.687 \\
\hline$(5,1,7,3)$ & 0.874 & 1.856 & 0.875 & 1.859 \\
\hline
\end{tabular}

Table 3.4: Analytical results vs state-space representations: the two-machine model 


\begin{tabular}{|c|c|c|c|c|}
\hline$\left(b_{1}, b_{2}, \lambda, \mu_{1}, \mu_{2}, \mu_{3}\right)$ & \multicolumn{2}{|c|}{ Analytical Method } & \multicolumn{2}{c|}{ Markov Chain } \\
\hline \hline & Mean & Variance & Mean & Variance \\
\hline$(0,0,1,3,3,3)$ & 0.239 & 1.832 & 0.241 & 1.833 \\
\hline$(0,0,1,6,4,3)$ & 0.274 & 2.000 & 0.275 & 1.999 \\
\hline$(2,2,1,3,3,3)$, & 0.282 & 0.128 & 0.285 & 0.131 \\
\hline$(2,2,1,6,4,3)$ & 0.392 & 0.164 & 0.388 & 0.163 \\
\hline$(2,5,1,6,4,3)$, & 0.401 & 0.207 & 0.398 & 0.205 \\
\hline$(5,2,1,6,4,3)$, & 0.408 & 0.209 & 0.409 & 0.211 \\
\hline$(5,5,1,6,4,3)$ & 0.412 & 0.214 & 0.412 & 0.213 \\
\hline
\end{tabular}

Table 3.5: Analytical results vs state-space representations: the three-machine model

The design used for the experiments is the paired comparison design in which the precision can be greatly improved by making comparisons within pairs of experimental results at a significance level of $95 \%$. The results of the experimental design show that there is no evidence to indicate that the two approaches produce statistically significant difference in the estimation of performance measures, which are the mean and the variance of the throughput rate.

\section{Simulation}

The simulation analysis helps to determine whether the model operates appropriately in the transient state. The codes to simulate the systems, which are developed under exactly the same assumptions for the analytical models, are written in the SIMAN simulation language (Pegden, Shannon, and Sadowski [185]). While obtaining the numerical results for the mean and variance of throughput rate at arbitrary instants in time via the use of simulation models, the following formulas are used:

$$
\mathrm{E}\left(\mathrm{N}_{\mathrm{n}}(\mathrm{t})\right)=\sum_{y=1}^{500} \frac{\mathrm{N}_{n}^{y}(\mathrm{t})}{500} \quad \text { and } \quad \operatorname{Var}\left(N_{n}(t)\right)=\sum_{y=1}^{500} \frac{\left.N_{n}^{y}(t)-E(X)\right)^{2}}{499},
$$

where $N_{n}^{y}(t)$ corresponds to the number of parts leaving the $n$-machine system at time $t$ in the $y^{t h}$ replication. These formulas are basically the definitions of the sample mean and the sample variance that are available in the reference of 
Montgomery [158]. Then, the mean and variance of throughput rate of the $n$ machine transfer line can be numerically calculated by $E(t)=E\left[\frac{N_{n}(t)}{t}\right]=\frac{E\left[N_{n}(t)\right]}{t}$ and $V(t)=V\left[\frac{N_{n}(t)}{t}\right]=\frac{V\left[N_{n}(t)\right]}{t^{2}}$, respectively, at time $t$.

\begin{tabular}{|c|c|c|c|c|}
\hline$(\lambda, \mu, t)$ & \multicolumn{2}{|c|}{ Analytical Method } & \multicolumn{2}{c|}{ Simulation } \\
\hline \hline & Mean & Variance & Mean & Variance \\
\hline$(2,20,2)$ & 1.802 & 1.422 & 1.809 & 1.431 \\
\hline$(2,20,5)$ & 1.816 & 1.434 & 1.824 & 1.438 \\
\hline$(2,20,10)$ & 1.882 & 1.496 & 1.885 & 1.501 \\
\hline$(2,20,25)$ & 1.844 & 1.450 & 1.846 & 1.451 \\
\hline$(2,20,50)$ & 1.837 & 1.451 & 1.838 & 1.449 \\
\hline$(2,20,100)$ & 1.828 & 1.451 & 1.827 & 1.449 \\
\hline
\end{tabular}

Table 3.6: Analytical results vs simulation: the atomic model
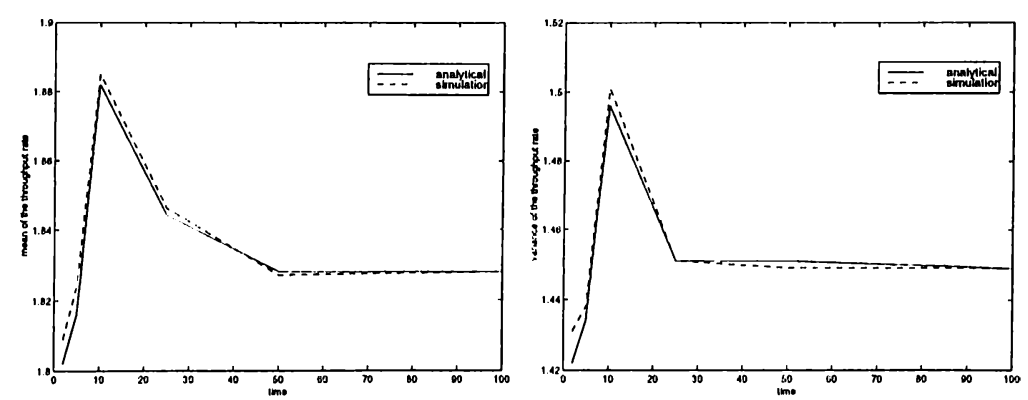

Figure 3.5: Mean and Variance vs time: the atomic model

It is observed that the pattern which analytical results follow is smoother than the one of the simulation results (Figures 3.5-3.7). This is due to the random number generator that is one of the main mechanism of the simulation software.

The simulation analysis indicates that the proposed method works fairly well in reflecting the transient behavior of the systems under consideration. This is confirmed by the paired comparison design that is done at a significance level of $95 \%$ with the result that the analytical and simulation based approach do not produce statistically significant difference in the estimation of mean and variance of throughput rate.

Finally, I must note the two important findings via the use of the figures 


\begin{tabular}{|c|c|c|c|c|}
\hline$\left(b_{1}, \lambda, \mu_{1}, \mu_{2}, t\right)$ & \multicolumn{2}{|c|}{ Analytical Method } & \multicolumn{2}{c|}{ Simulation } \\
\hline \hline & Mean & Variance & Mean & Variance \\
\hline$(5,1,3,3,2)$ & 0.869 & 0.318 & 0.872 & 0.320 \\
\hline$(5,1,3,3,5)$ & 0.801 & 0.267 & 0.794 & 0.270 \\
\hline$(5,1,3,3,10)$ & 0.772 & 0.242 & 0.776 & 0.249 \\
\hline$(5,1,3,3,25)$ & 0.761 & 0.238 & 0.762 & 0.239 \\
\hline$(5,1,3,3,50)$ & 0.755 & 0.235 & 0.752 & 0.238 \\
\hline$(5,1,3,3,100)$ & 0.743 & 0.231 & 0.742 & 0.231 \\
\hline$(5,1,3,3,250)$ & 0.732 & 0.227 & 0.732 & 0.227 \\
\hline$(5,1,3,3,500)$ & 0.732 & 0.227 & 0.732 & 0.227 \\
\hline
\end{tabular}

Table 3.7: Analytical results vs simulation: the two-machine model
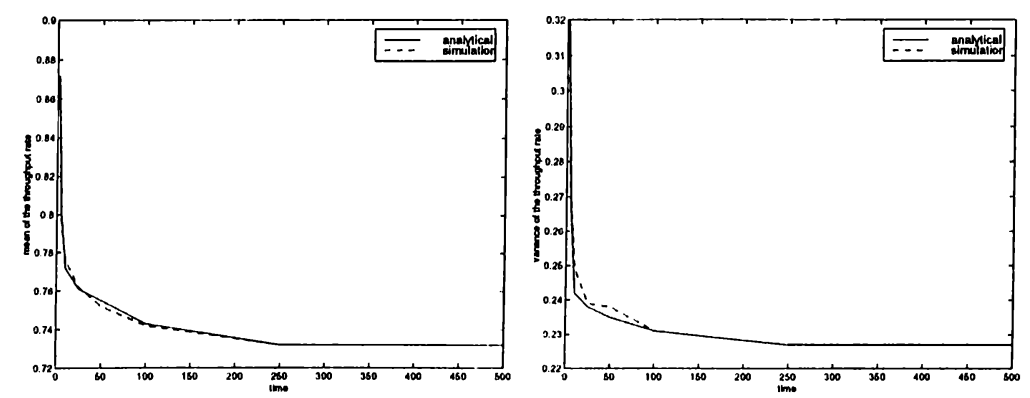

Figure 3.6: Mean and Variance vs time: the two-machine model

3.5-3.7. The first one is that the existence of the buffers in a system smooths out the pattern indicating the behavior of the throughput in time. Observe that the patterns of the mean and variance of the throughput in the single machine system (figure 3.5) are sharper than those of the relevant measures in the longer lines (figures 3.6-3.7). This observation is due to the description of the relevant systems. While the single-machine system has zero buffer capacity (i.e., $b_{0}=0$ ), the two- and three-machine systems have finite buffer storages, respectively (i.e., $b_{1}, b_{2}>0$ ). The second finding is that the throughput rate decreases in time while the contrary holds for longer lines (observe how the throughput rate changes in time for the two-machine system (Figure 3.6) and for the three-machine system (figure 3.7)). The decrease in the throughput rate is due to the definition of the mean and variance of the throughput rate (i.e., $E(t)=E\left[\frac{N_{n}(t)}{t}\right]=\frac{E\left[N_{n}(t)\right]}{t}$ and $\left.V(t)=V\left[\frac{N_{n}(t)}{t}\right]=\frac{V\left[N_{n}(t)\right]}{t^{2}}\right)$. The changes in the mean and variance of the throughput are smaller than the lengths of the 


\begin{tabular}{|c|c|c|c|c|}
\hline$\left(b_{1}, b_{2}, \lambda, \mu_{1}, \mu_{2}, \mu_{3}, t\right)$ & \multicolumn{2}{|c|}{ Analytical Method } & \multicolumn{2}{c|}{ Simulation } \\
\hline \hline & Mean & Variance & Mean & Variance \\
\hline$(2,5,20,6,4,3,2)$ & 2.012 & 1.364 & 1.767 & 1.361 \\
\hline$(2,5,20,6,4,3,5)$ & 2.296 & 1.492 & 2.287 & 1.467 \\
\hline$(2,5,20,6,4,3,10)$ & 2.511 & 1.668 & 2.509 & 1.666 \\
\hline$(2,5,20,6,4,3,25)$ & 2.674 & 1.692 & 2.666 & 1.678 \\
\hline$(2,5,20,6,4,3,50)$ & 2.703 & 1.715 & 2.699 & 1.716 \\
\hline$(2,5,20,6,4,3,100)$ & 2.713 & 1.728 & 2.713 & 1.727 \\
\hline$(2,5,20,6,4,3,250)$ & 2.703 & 1.721 & 2.701 & 1.719 \\
\hline$(2,5,20,6,4,3,500)$ & 2.689 & 1.712 & 2.688 & 1.711 \\
\hline$(2,5,20,6,4,3,1000)$ & 2.689 & 1.712 & 2.689 & 1.712 \\
\hline
\end{tabular}

Table 3.8: Analytical results vs simulation: the three-machine model
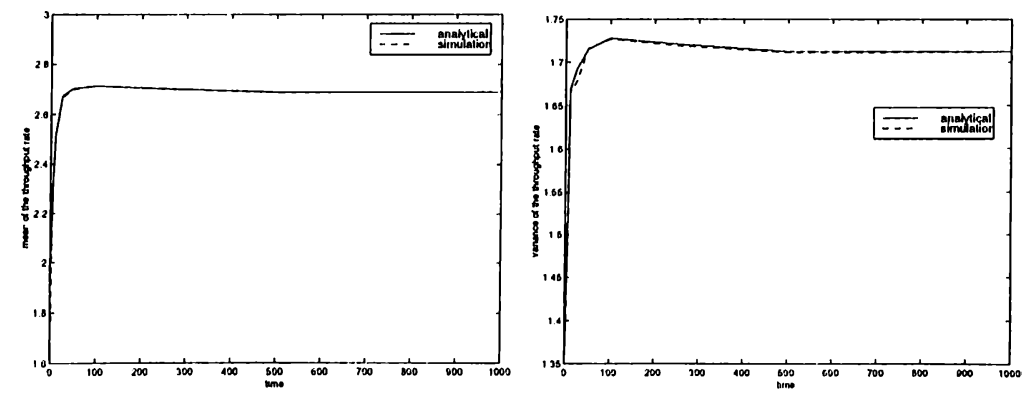

Figure 3.7: Mean and Variance vs time: the three-machine model

time intervals under consideration. However, as the number of machines in the system increase, significant increases in $E(t)$ and $V(t)$ are observed in time due to the additional buffers existing in the relevant systems. The more detailed results and discussion on the behavior of the throughput of transfer lines can be found in Deler and Dinçer [57].

\subsection{Summary}

In this section, the transient behavior of transfer lines with reliable machines and finite buffers is examined and the distribution of the performance measures of interest is derived. The results of the proposed analytical method are compared with the state-space representations and the simulation for validation. Both transient and steady-state behavior of this system 
are determined by using the evolution of the stochastic processes under consideration. The proposed method based on the analytical derivation of the distribution of the throughput of the system provides good results for typical transfer lines (up to three stages) encountered in real applications. The detailed results are given in Appendix A.3. 


\section{Chapter 4}

\section{Extension of the Method: Two-Part Types}

\subsection{Introduction}

In the general context, a multiple-part-type tandem system is a $n$-machine, $m$-part-type tandem production line. The system consists of $n$ machines and $(n+1) m$ buffers. Type $i$ parts travel in a fixed sequence: Buffer $(0, i)$, Machine 1, Buffer $(1, i)$, Machine 2, Buffer $(2, i), \ldots ., \operatorname{Buffer}(n-1, i)$, Machine $n$, Buffer $(n, i)$. Buffers are assumed homogeneous. That is, $\operatorname{Buffer}(j, i)$ holds only identical, interchangeable parts: Type $i$ parts that have completed operations on Machine $j$ and are waiting for an operation on Machine $j+1$.

This brief explanation about the configuration of the multiple-part-type tandem systems indicates that such a system is difficult to analyze due to the increase in the number of the additional sources of randomness which are the part types and their associated buffers. However, transfer lines are common in industries such as food, automotive, electronics, and pharmaceutical, among many others (Altiok [11]). By means of the development in the machine design, any machine can act as a single-server attending the requests for production of different types of products with its own pool of production orders. In such a multi-product environment, there are two important issues, which are "when 
to switch to a new product" and "what product to switch to". While the first issue can be handled by an $(\mathrm{R}, \mathrm{r})$-type inventory control policy, the second one can be resolved by using priorities. However, the literature makes the assumption that the relevant systems are in their steady-states. As discussed in Chapter 1, since the time horizon of operation naturally terminates, steadystate measures of system simply do not make sense leading to the important conclusion that such steady-state analyses are inappropriate in many applied situations. Therefore, the models and solution methods that could capture both the transient behavior of the $n$-machine, $m$-part type tandem production line should be investigated. In this chapter, we extend the new method to the typical transfer lines (up to three stages) with two-part types.

\subsubsection{The model assumptions and notation}

Before going into details, it will be beneficial to state the major assumptions: The assurnptions listed in Table 3.1 are still valid, however, there are some items to be added to the list (Table 4.1).

Table 4.1: The additional assumptions

$1^{*}$. The two-part type production line is a serial arrangement of a finite number of $n$ machines and 2(n-1) buffers. Each machine can operate on one unit of product at a time and has internal storage capacity for that unit.

11. All buffers are assumed homogeneous. That is, $\operatorname{Buffer}(j, i)$ holds only identical, interchangeable parts: Type $i$ parts that have completed operations on Machine $j$ and are waiting for an operation on Machine $j+1$.

12. Parts leave the buffers to be processed in the downstream machines according to the order of their arrivals.

The following notation is introduced as addition to the ones listed in Table 4.2. 
Table 4.2: The additional notation

$p^{j, k}: \quad$ number of parts of type $j$ arriving the system in the $k^{\text {th }}$ period

$p_{c}^{j, k}$ : number of parts of type $j$ arriving the system under Case $c$ in the $k^{t h}$ period

$T_{\lambda}^{j, i}:$ inter-arrival time for the $i^{\text {th }}$ part of type $j$

$T_{\mu_{s}}^{j, i}: \quad$ service time for the $i^{t h}$ part of type $j$ on machine $s, s=1, \ldots, \mathrm{n}$

$\mu_{s j} \quad$ service rate of machine $s$ for part type $j, s=1, \ldots, \mathrm{n}$

$b_{1 j}$ : capacity of the buffer between the first and second machines holding part type $j$

$b_{2 j}$ : capacity of the buffer between the second and third machines holding part type $j$

\subsubsection{Organization}

In this chapter, we extend the analytical method for estimating the performance measures of a serial production line with reliable machines and finite buffers to the systems with two-part types. First section is devoted to the model development. In this section, the evolutions of the stochastic processes of the random variable $N_{1}(t)$ for the atomic model, $N_{2}(t)$ for the two-machine-onebuffer system, and $N_{3}(t)$ for the three-machine-two-buffer system, respectively, and the corresponding analytical derivations are determined. The final section consists of an experimental design that helps to determine whether the model can capture the true transient and steady-state behaviors of the corresponding systems.

\subsection{The model development}

\subsubsection{Modeling}

This section gives the verbal and graphical descriptions of the transient behavior of the systems. The corresponding descriptions help us to write down the representations for the number of parts leaving the systems at arbitrary instants in time. 
CHAPTER 4. EXTENSION OF THE METHOD: TWO-PART TYPES 68

The atomic model

The atomic model corresponds to one-machine system as depicted in Figure 4.1:

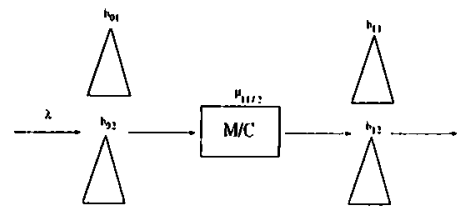

The evolution of the stochastic process of the random variable, $N_{1}(t)$, is presented below.

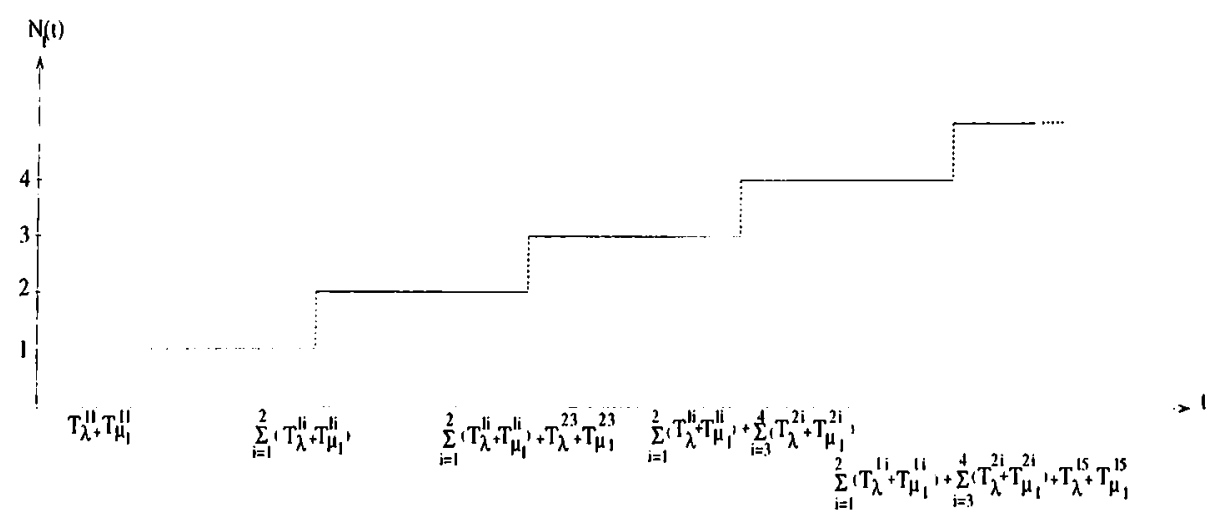

Figure 4.2: Evolution of the stochastic process, $N_{1}(t)$.

The number of parts leaving the atomic model at time $t, N_{1}(t)$, can be written as

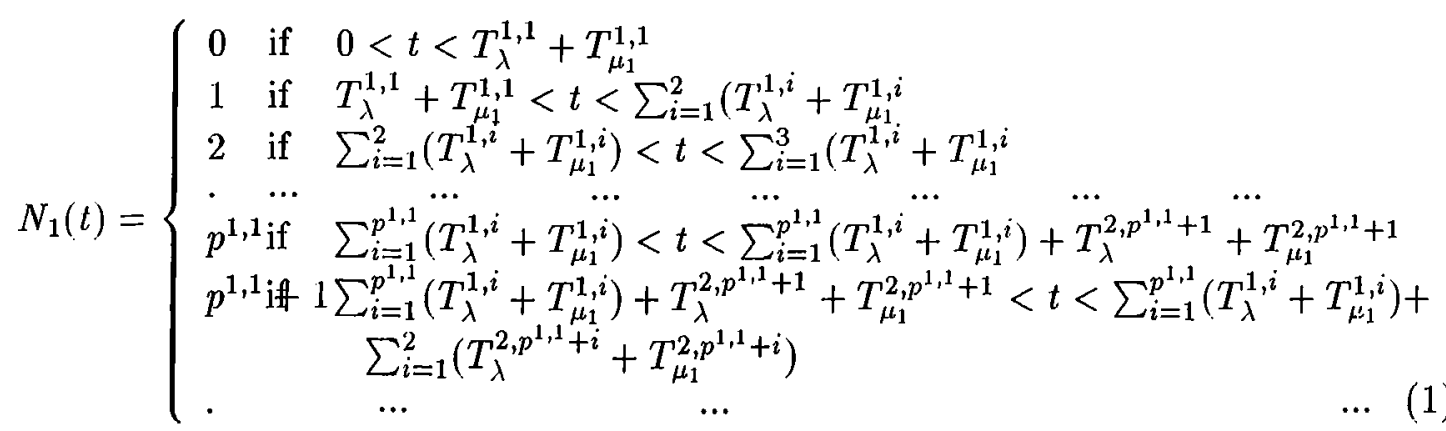




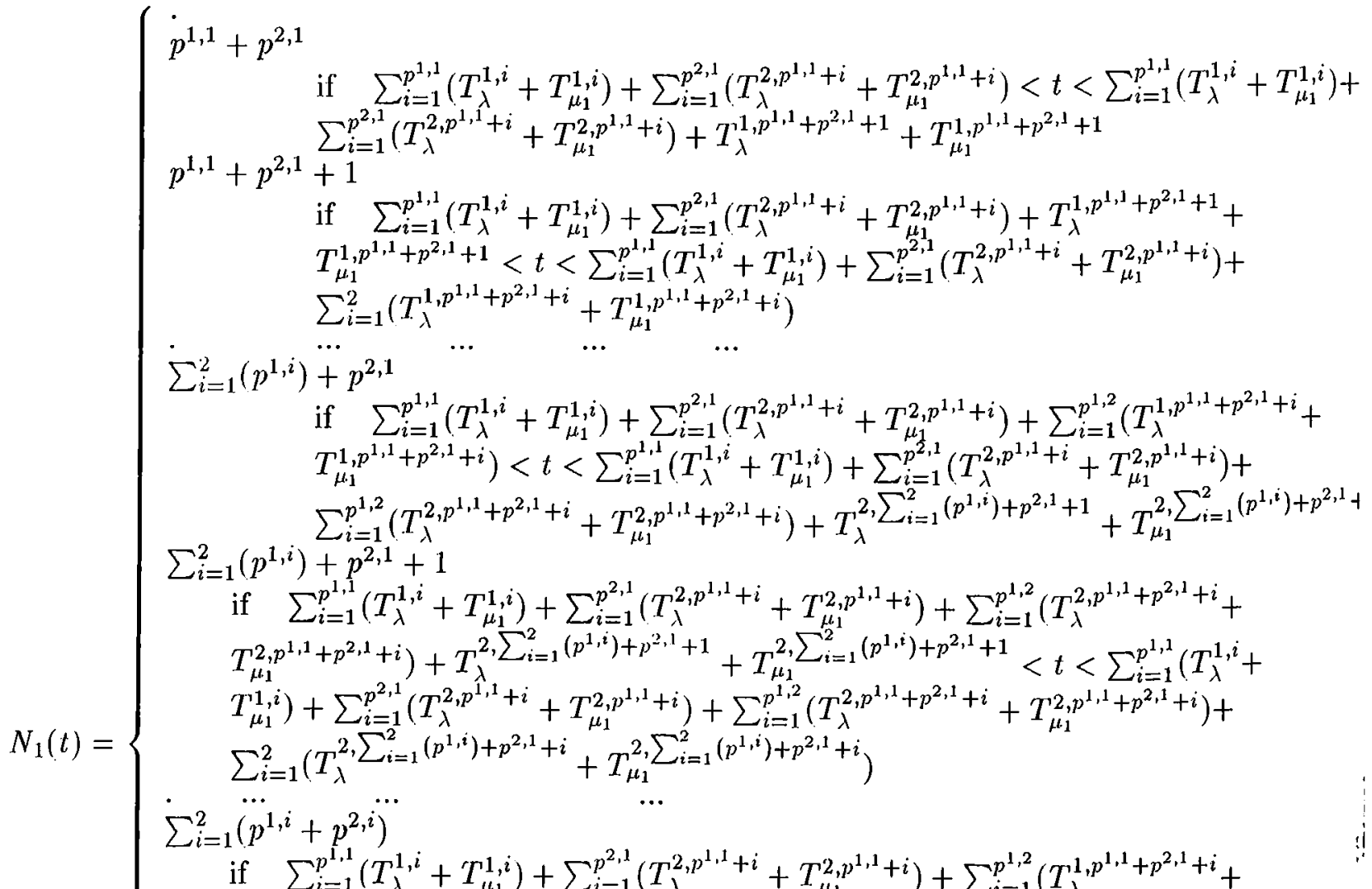

$$
\begin{aligned}
& \left.T_{\mu_{1}}^{1, p^{1,1}+p^{2,1}+i}\right)+\sum_{i=1}^{p^{2,2}}\left(T_{\lambda}^{2, \sum_{i=1}^{2}}\left(p^{1, i}\right)+p^{2,1}+i+T_{\mu_{1}}^{2, \sum_{i=1}^{2}\left(p^{1, i}\right)+p^{2,1}+i}\right)<t< \\
& \sum_{i=1}^{p^{1,1}}\left(T_{\lambda}^{1, i}+T_{\mu_{1}}^{1, i}\right)+\sum_{i=1}^{p^{2,1}}\left(T_{\lambda}^{2, p^{1,1}+i}+T_{\mu \mu_{1}}^{2, p^{1,1}+i}\right)+\sum_{i=1}^{p^{1,2}}\left(T_{\lambda}^{1, p^{1,1}+p^{2,1}+i}+\right. \\
& \left.T_{\mu_{1}}^{1, p^{1,1}+p^{2,1}+i}\right)+\sum_{i=1}^{p^{2,2}}\left(T_{\lambda}^{2, \sum_{i=1}^{2}\left(p^{1, i}\right)+p^{2,1}+i}+T_{\mu_{1}}^{2, \sum_{i=1}^{2}\left(p^{1, i}\right)+p^{2,1}+i}\right)+ \\
& T_{\lambda}^{1, \sum_{i=1}^{2}\left(p^{1, i}+p^{2, i}\right)+1}+T_{\mu_{1}}^{1, \sum_{i=1}^{2}\left(p^{1, i}+p^{2, i}+1\right)} \\
& \left.\sum_{i=1}^{k} \ddot{\left(p^{1, i}\right.}+p^{2, i}\right) \\
& \text { if } \sum_{i=1}^{k}\left(\sum_{j=1}^{p^{1, i}}\left(T_{\lambda}^{1, \sum_{l=1}^{i-1}\left(p^{1, l}+p^{2, l}\right)+j}+T_{\mu_{1}}^{1, \sum_{l=1}^{i-1}\left(p^{1, l}+p^{2, l}\right)+j}\right)+\right. \\
& \left.\sum_{j=1}^{p^{2, i}}\left(T_{\lambda}^{2, \sum_{l=1}^{i}\left(p^{1, l}\right)+\sum_{l=1}^{i-1}\left(p^{2, l}\right)+j}+T_{\mu_{1}}^{2, \sum_{l=1}^{i}\left(p^{1, l}\right)+\sum_{l=1}^{i-1}\left(p^{2, l}\right)+j}\right)\right)<t< \\
& \sum_{i=1}^{k}\left(\sum_{j=1}^{p^{1, i}}\left(T_{\lambda}^{1, \sum_{l=1}^{i-1}\left(p^{1, l}+p^{2, l}\right)+j}+T_{\mu_{1}}^{1, \sum_{l=1}^{i-1}\left(p^{1, l}+p^{2, l}\right)+j}\right)+\right. \\
& \left.\sum_{j=1}^{p^{2, i}}\left(T_{\lambda}^{2, \sum_{l=1}^{i}\left(p^{1, l}\right)+\sum_{l=1}^{i-1}\left(p^{2, l}\right)+j}+T_{\mu_{1}}^{2, \sum_{l=1}^{i}\left(p^{1, l}\right)+\sum_{l=l}^{i-1}\left(p^{2, l}\right)+j}\right)\right)+ \\
& \left.T_{\lambda}^{1, \sum_{i=1}^{k}\left(p^{1, i}+p^{2, i}\right)+1}+T_{\mu_{1}}^{1, \sum_{i=1}^{k}\left(p^{1, i}+p^{2, i}\right)+1}\right)
\end{aligned}
$$




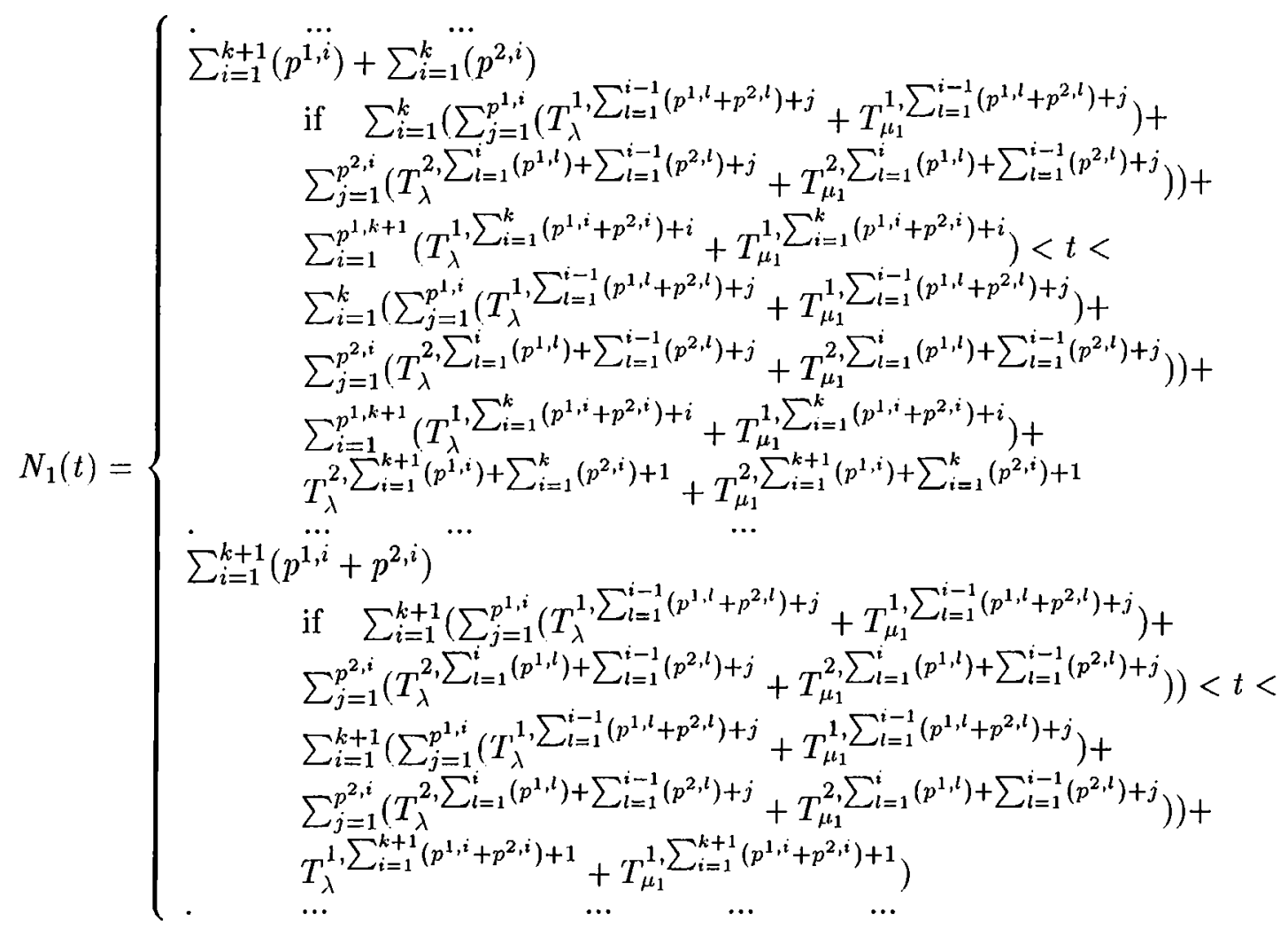

Similarly, the assumptions that facilitate the representation of the stochastic process evolution in such a clear and neat way are the memorylessness property of the exponential distribution and zero buffer capacity of the first buffer in the line. Moreover, the number of parts of different types arriving the system are known in advance. In case of a sudden change in the order of part types' arrivals to the system, the method brings the flexibility of recalculation of the performance measures via the use of parameter $p^{j, k}$, that is the number of parts of type $j$ arriving the system in the $k^{\text {th }}$ period.

\section{Two-machine-one-buffer system}

The system under consideration is illustrated in Figure 4.3:

In this system, the sources of variability turn out to be $T_{\lambda}^{j, i}, T_{\mu_{1}}^{j, i}, T_{\mu_{2}}^{j, i}$, and $b_{1 j}$ for each part $i$ of type $j$ and this leads to the existence of the following mutually exclusive and collectively exhaustive events that describe the behavior of the 


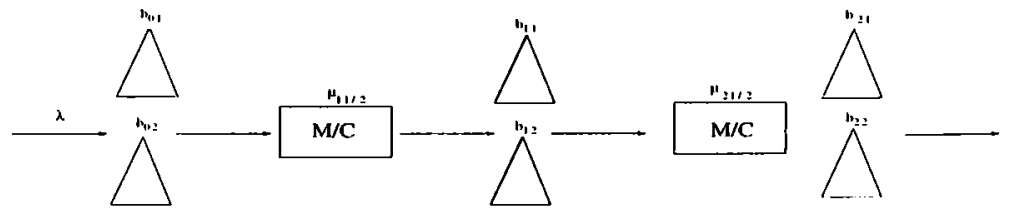

Figure 4.3: $T_{\lambda}^{j, i} \sim \exp (\lambda), T_{\mu_{s j}}^{j, i} \sim \exp \left(\mu_{s j}\right) \forall i \& j=1,2, \mathrm{~s}=1,2 ; \mathrm{b}_{0 j}=0, b_{1 j} \geq 0$, $\& b_{2 j}=\infty j=1,2$.

system : $T_{\lambda}^{j, i}+T_{\mu_{1}}^{j, i}>T_{\mu_{2}}^{j, i-1}$ and $T_{\lambda}^{j, i}+T_{\mu_{1}}^{j, i} \leq T_{\mu_{2}}^{j, i-1}$ for each part $i$ of type $j$. In the first case, no part accumulates in the buffer. In the second one, the first machine gets blocked some time depending on the relative magnitudes of the inter-arrival times and the service times of the machines, and also the capacity of the second buffer of the line. Since the consideration of these mutually exclusive and collectively exhaustive descriptions of the system behavior does not capture the true system behavior, an aggregate case is considered under which each condition is let be valid for particular number of parts arriving the system. In this way, we obtain the evolution of the stochastic process in which the strict assumptions of the basic conditions are relaxed.

The analytical derivations for $N_{2}(t)$ under Case 1, Case 2, which are the basic descriptions of the system behavior for the two-machine transfer line with finite buffer storage, are given in the Appendix B.1.

\section{Three-machine-two-buffer system}

The system under consideration is illustrated in Figure 4.4:

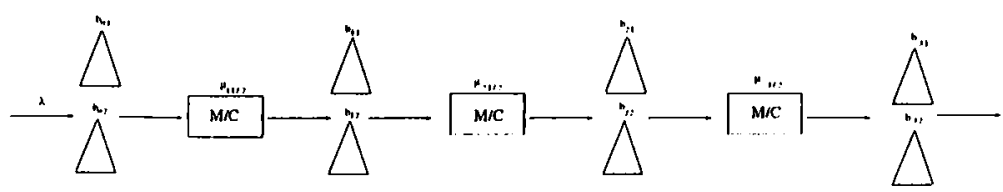

Figure 4.4: $T_{\lambda}^{j, i} \sim \exp (\lambda), T_{\mu_{s j}}^{j, i} \sim \exp \left(\mu_{s j}\right) \forall i, j=1,2, \mathrm{~s}=1,2,3 ; \mathrm{b}_{0 j}=0$, $b_{s j} \geq 0, \& b_{3 j}=\infty, j=1,2 \& s=1,2$.

In this system, there arises the following mutually exclusive and collectively exhaustive events representing the behavior of the three-machine transfer line: 
$T_{\lambda}^{j, i}+T_{\mu_{1}}^{j, i} \geq T_{\mu_{2}}^{j, i-1}$ and $T_{\lambda}^{j, i}+T_{\mu_{1}}^{j, i}+T_{\mu_{2}}^{j, i} \geq T_{\mu_{2}}^{j, i-1}+T_{\mu_{3}}^{j, i-1}, T_{\lambda}^{j, i}+T_{\mu_{1}}^{j, i} \geq T_{\mu_{2}}^{j, i-1}$ and $T_{\lambda}^{j, i}+T_{\mu_{1}}^{j, i}+T_{\mu_{2}}^{j, i}<T_{\mu_{2}}^{j, i-1}+T_{\mu_{3}}^{j, i-1}, T_{\lambda}^{j, i}+T_{\mu_{1}}^{j, i}<T_{\mu_{2}}^{j, i-1}$ and $T_{\mu_{3}}^{j, i-2} \geq T_{\mu_{2}}^{j, i-1}$, and $T_{\lambda}^{j, i}+T_{\mu_{1}}^{j, i}<T_{\mu_{2}}^{j, i-1}$ and $T_{\mu_{3}}^{j, i-2}<T_{\mu_{2}}^{j, i-1}$ for each part of type $j$. Since none of the conditions uniquely represent the true system behavior by itself, similarly, each condition is allowed to be valid for particular number of parts in each period under the aggregate case, which are assumed to be known in advance.

The analytical derivations for $N_{3}(t)$ under Case 1, Case 2, Case 3, Case 4, which are the basic descriptions of the system behavior for three-machine transfer line with finite buffer storages, are also given in the Appendix B.2.

\subsubsection{Approach}

In the previous section, the evolution of the stochastic processes of interest are given. The way in which the corresponding characterizations of the distribution of the output process of the systems under consideration is utilized to obtain the moments of throughput, is exactly the same way as expressed in Section 3.2.2. The expressions for $P\left(N_{n}(t)=l\right), n=1,2,3$ and $l=0,1,2, \ldots$ are deduced from the available analytical derivations. Similarly, substitution of $P\left(N_{n}(t)=l\right), n=1,2,3$ and $l=0,1,2, \ldots$. into $E\left[N_{n}(t)\right]=\sum_{l=1}^{\infty} l \times P\left(N_{n}(t)=l\right)$ enables us to obtain the analytical representation of the mean of the number of parts leaving the $n$-machine transfer line. Furthermore, $V\left[N_{n}(t)\right]$, variance of the number of units leaving the $n$-machine transfer line, can be obtained both analytically and numerically by substituting the relevant expressions into $E\left[N_{n}(t) \times\left(N_{n}(t)-1\right)\right]+E\left[N_{n}(t)\right]-E\left[N_{n}(t)\right]^{2}$.

\subsection{Numerical Results}

Succeeding sections is devoted to the explanation of the coding environment and the numerical results concerning the approximation accuracy. Moreover, an experiment is designed to determine whether the models can capture the true transient and steady-state behaviors of the corresponding systems. 


\subsubsection{Computer Codes}

The coding environment of the proposed method for the two-part type system is similar to the one mentioned in Section 3.3.1.

\subsubsection{Validation}

After the development of the model, an experiment is designed in order to determine whether the model can capture the true transient and steady-state behaviors of the corresponding systems.

\section{State-space representation}

For each system, the mean and the variance of the throughput rate obtained by the analytical method, are compared with the ones given by the statespace representations developed under exactly the same assumptions for the analytical models (Tables 4.3-4.5).

\begin{tabular}{|c|c|c|c|c|}
\hline$\left(\lambda, \mu_{1}^{1}, \mu_{1}^{2}\right)$ & \multicolumn{2}{|c|}{ Analytical Method } & \multicolumn{2}{c|}{ Markov Chain } \\
\hline \hline & Mean & Variance & Mean & Variance \\
\hline$(3,5,5)$ & 2.855 & 5.622 & 2.856 & 5.622 \\
\hline$(3,2,7)$ & 2.045 & 3.678 & 2.045 & 3.675 \\
\hline$(3,7,2)$ & 1.729 & 2.859 & 1.731 & 2.857 \\
\hline
\end{tabular}

Table 4.3: Analytical results vs state-space representations: the atomic model

\begin{tabular}{|c|c|c|c|c|}
\hline$\left(b_{1}^{1}, b_{1}^{2}, \lambda, \mu_{1}^{1}, \mu_{1}^{2}, \mu_{2}^{1}, \mu_{2}^{2}\right)$ & \multicolumn{2}{|c|}{ Analytical Method } & \multicolumn{2}{c|}{ Markov Chain } \\
\hline \hline & Mean & Variance & Mean & Variance \\
\hline$(0,0,1,3,3,3,3)$ & 0.798 & 1.376 & 0.797 & 1.375 \\
\hline$(0,0,1,7,3,7,3)$ & 0.972 & 1.525 & 0.972 & 1.524 \\
\hline$(2,2,1,3,3,3,3)$ & 0.812 & 1.436 & 0.812 & 1.436 \\
\hline$(2,2,1,7,3,7,3)$ & 1.003 & 1.572 & 1.002 & 1.572 \\
\hline$(5,5,1,3,3,3,3)$ & 0.934 & 1.499 & 0.933 & 1.501 \\
\hline$(5,5,1,7,3,7,3)$ & 1.038 & 1.634 & 1.038 & 1.635 \\
\hline
\end{tabular}

Table 4.4: Analytical results vs state-space representations: the two-machine model 


\begin{tabular}{|c|c|c|c|c|}
\hline$\left(b_{1}^{1}, b_{1}^{2}, b_{2}^{1}, b_{2}^{2}, \lambda, \mu_{1}^{1}, \mu_{1}^{2}, \mu_{2}^{1}, \mu_{2}^{2}, \mu_{3}^{1}, \mu_{3}^{2}\right)$ & \multicolumn{2}{|c|}{ Analytical Method } & \multicolumn{2}{c|}{ Markov Chain } \\
\hline \hline & Mean & Variance & Mean & Variance \\
\hline$(0,0,0,0,1,3,3,3,3,3,3)$ & 0.552 & 1.226 & 0.553 & 1.226 \\
\hline$(0,0,0,0,1,6,2,4,7,3,6)$ & 0.635 & 1.108 & 0.634 & 1.009 \\
\hline$(2,2,2,2,1,3,3,3,3,3,3)$ & 0.629 & 0.903 & 0.632 & 0.905 \\
\hline$(2,2,2,2,1,6,2,4,7,3,6)$ & 0.814 & 1.128 & 0.814 & 1.128 \\
\hline$(2,2,5,5,1,6,2,4,7,3,6)$ & 0.738 & 1.102 & 0.741 & 1.105 \\
\hline$(5,5,2,2,1,6,2,4,7,3,6)$ & 0.957 & 1.214 & 0.957 & 1.214 \\
\hline$(5,5,5,5,1,6,2,4,7,3,6)$ & 0.412 & 0.214 & 0.412 & 0.213 \\
\hline
\end{tabular}

Table 4.5: Analytical results vs state-space representations: the three-machine model

The design used for the experiments is the paired comparison design in which the precision can be greatly improved by making comparisons within pairs of experimental results at a significance level of $95 \%$. The results of the experimental design show that there is no evidence to indicate that the two approaches produce statistically significant difference in the estimation of performance measures, which are the mean and the variance of the throughput rate.

\section{Simulation}

The simulation analysis helps to determine whether the model operates appropriately in the transient state. The codes to simulate the systems developed under exactly the same assumptions for the analytical models, are also written in the SIMAN simulation language (Pegden, Shannon, and Sadowski [185]). While obtaining the numerical results for the mean and variance of throughput at arbitrary instants in time via the use of simulation models, the following formulas are used:

$$
\mathrm{E}\left(\mathrm{N}_{\mathrm{n}}(\mathrm{t})\right)=\sum_{y=1}^{500} \frac{\mathrm{N}_{1}^{\mathrm{y}}(\mathrm{t})}{500} \quad \text { and } \quad \operatorname{Var}\left(N_{n}(t)\right)=\sum_{y=1}^{500} \frac{\left.N_{n}^{y}(t)-E(X)\right)^{2}}{499},
$$

where $N_{n}^{y}(t)$ corresponds to the number of parts leaving the $n$-machine system at time $t$ in the $y^{t h}$ replication. These formulas are basically the definitions of the sample mean and the sample variance that are available in the reference of Montgomery [158]. Then, the mean and variance of throughput rate rate of 
the $n$-machine transfer line can be numerically calculated by $E(t)=E\left[\frac{N_{n}(t)}{t}\right]=$ $\frac{E\left[N_{n}(t)\right]}{t}$ and $V(t)=V\left[\frac{N_{n}(t)}{t}\right]=\frac{V\left[N_{n}(t)\right]}{t^{2}}$, respectively, at time $t$.

\begin{tabular}{|c|c|c|c|c|}
\hline$\left(\lambda, \mu_{1}^{1}, \mu_{1}^{2}, t\right)$ & \multicolumn{2}{|c|}{ Analytical Method } & \multicolumn{2}{c|}{ Simulation } \\
\hline \hline & Mean & Variance & Mean & Variance \\
\hline$(2,2,5,2)$ & 1.995 & 1.376 & 1.996 & 1.375 \\
\hline$(2,2,5,5)$ & 1.968 & 1.377 & 1.968 & 1.376 \\
\hline$(2,2,5,10)$ & 1.962 & 1.333 & 1.961 & 1.335 \\
\hline$(2,2,5,25)$ & 1.955 & 1.355 & 1.954 & 1.355 \\
\hline$(2,2,5,50)$ & 1.957 & 1.357 & 1.956 & 1.357 \\
\hline$(2,2,5,100)$ & 1.962 & 1.358 & 1.961 & 1.359 \\
\hline$(2,2,5,250)$ & 1.983 & 1.362 & 1.982 & 1.361 \\
\hline$(2,2,5,300)$ & 1.982 & 1.362 & 1.982 & 1.362 \\
\hline
\end{tabular}

Table 4.6: Analytical results vs simulation: the atomic model

The simulation analysis indicates that the proposed method works fairly well in reflecting the transient behavior of the systems under consideration (Tables 4.6-4.8 and figures 4.5-4.7). This is confirmed by the paired comparison design that is done at a significance level of $95 \%$ with the result that the analytical and simulation based approach do not produce statistically significant difference in the estimation of mean and variance of throughput rate.
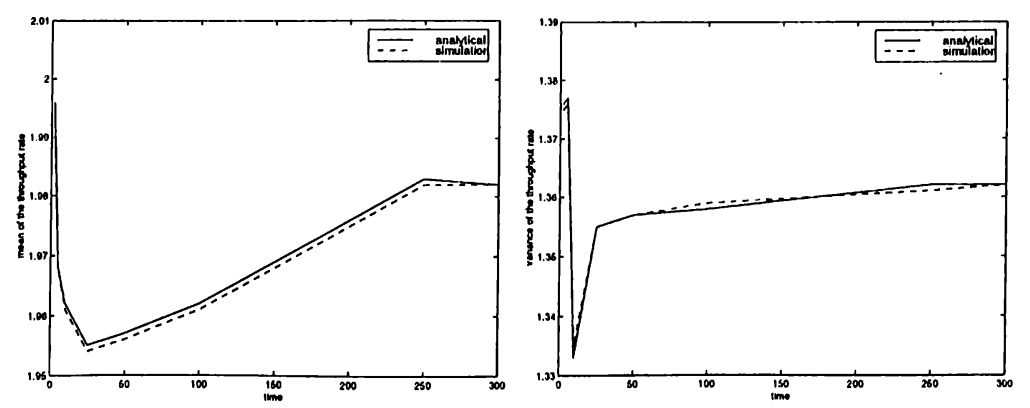

Figure 4.5: Mean and Variance vs time: the atomic model

As noted in the previous chapter, the two important findings on the behavior of throughput of transfer lines also hold for multiple-part type 


\begin{tabular}{|c|c|c|c|c|}
\hline$\left(b_{1}^{1}, b_{1}^{2}, \lambda, \mu_{1}^{\mathbf{1}}, \mu_{1}^{2}, \mu_{2}^{1}, \mu_{2}^{2}, t\right)$ & \multicolumn{2}{|c|}{ Analytical Method } & \multicolumn{2}{c|}{ Simulation } \\
\hline \hline & Mean & Variance & Mean & Variance \\
\hline$(5,5,1,3,4,3,4,2)$ & 3.744 & 2.405 & 3.745 & 2.401 \\
\hline$(5,5,1,3,4,3,4,5)$ & 3.106 & 2.379 & 3.304 & 2.362 \\
\hline$(5,5,1,3,4,3,4,10)$ & 3.234 & 2.096 & 3.235 & 2.092 \\
\hline$(5,5,1,3,4,3,4,25)$ & 2.842 & 1.972 & 2.842 & 1.974 \\
\hline$(5,5,1,3,4,3,4,50)$ & 2.668 & 1.886 & 2.668 & 1.885 \\
\hline$(5,5,1,3,4,3,4,100)$ & 2.591 & 1.728 & 2.590 & 1.727 \\
\hline$(5,5,1,3,4,3,4,250)$ & 2.367 & 1.438 & 2.366 & 1.437 \\
\hline$(5,5,1,3,4,3,4,500)$ & 2.035 & 1.257 & 2.034 & 1.256 \\
\hline$(5,5,1,3,4,3,4,600)$ & 2.048 & 1.023 & 2.048 & 1.022 \\
\hline$(5,5,1,3,4,3,4,750)$ & 2.048 & 1.023 & 2.048 & 1.023 \\
\hline
\end{tabular}

Table 4.7: Analytical results vs simulation: the two-machine model
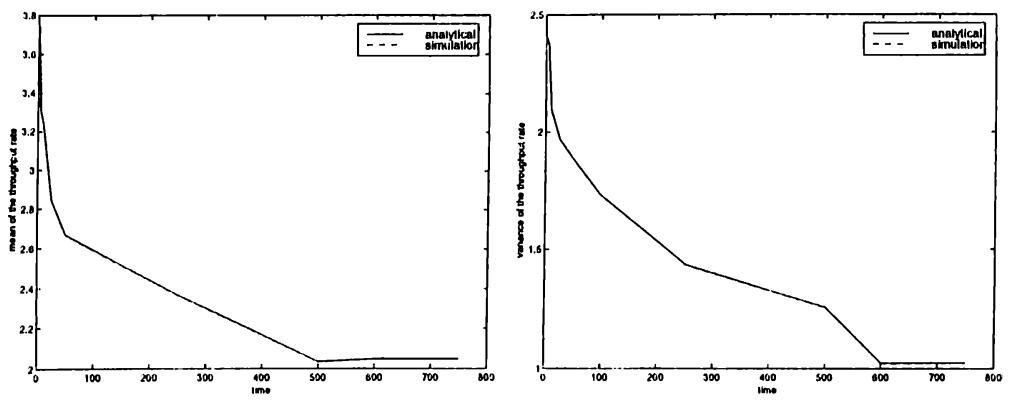

Figure 4.6: Mean and Variance vs time: the two-machine model

systems. However, the length of the transient period for a multiple-part type system is larger than that of the single-part type system due to the considerable amount of variability introduced to the system by both the processing times of different part types and the fluctuations in the buffer levels (Deler and Sabuncuoglu [58]). The more detailed results and discussion on the behavior of throughput of transfer lines can also be found in Deler and Dinçer [57].

\subsection{Summary}

In this section, the new method introduced in the previous chapter is extended to the systems with two-part types. Both transient and steady-state behavior of this system are determined by using the evolution of the stochastic processes 


\begin{tabular}{|c|c|c|c|c|}
\hline$\left(b_{1}^{1}, b_{1}^{2}, b_{2}^{1}, b_{2}^{2}, \lambda, \mu_{1}^{1}, \mu_{1}^{2}, \mu_{2}^{1}, \mu_{2}^{2}, \mu_{3}^{1}, \mu_{3}^{2}, t\right)$ & \multicolumn{2}{|c|}{ Analytical Method } & \multicolumn{2}{|c|}{ Simulation } \\
\hline \hline & Mean & Variance & Mean & Variance \\
\hline$(2,2,5,5,20,6,2,4,7,3,6,2)$ & 3.116 & 3.123 & 3.115 & 3.125 \\
\hline$(2,2,5,5,20,6,2,4,7,3,6,5)$ & 3.142 & 3.224 & 3.141 & 3.225 \\
\hline$(2,2,5,5,20,6,2,4,7,3,6,10)$ & 3.178 & 3.435 & 3.179 & 3.434 \\
\hline$(2,2,5,5,20,6,2,4,7,3,6,25)$ & 3.185 & 3.538 & 3.185 & 3.538 \\
\hline$(2,2,5,5,20,6,2,4,7,3,6,50)$ & 3.185 & 3.677 & 3.187 & 3.678 \\
\hline$(2,2,5,5,20,6,2,4,7,3,6,100)$ & 3.196 & 3.873 & 3.195 & 3.873 \\
\hline$(2,2,5,5,20,6,2,4,7,3,6,250)$ & 3.204 & 3.879 & 3.201 & 3.876 \\
\hline$(2,2,5,5,20,6,2,4,7,3,6,500)$ & 3.212 & 3.902 & 3.211 & 3.901 \\
\hline$(2,2,5,5,20,6,2,4,7,3,6,1000)$ & 3.216 & 3.912 & 3.216 & 3.912 \\
\hline$(2,2,5,5,20,6,2,4,7,3,6,1200)$ & 3.221 & 3.909 & 3.220 & 3.908 \\
\hline$(2,2,5,5,20,6,2,4,7,3,6,1500)$ & 3.221 & 3.909 & 3.221 & 3.909 \\
\hline
\end{tabular}

Table 4.8: Analytical results vs simulation: the three-machine model
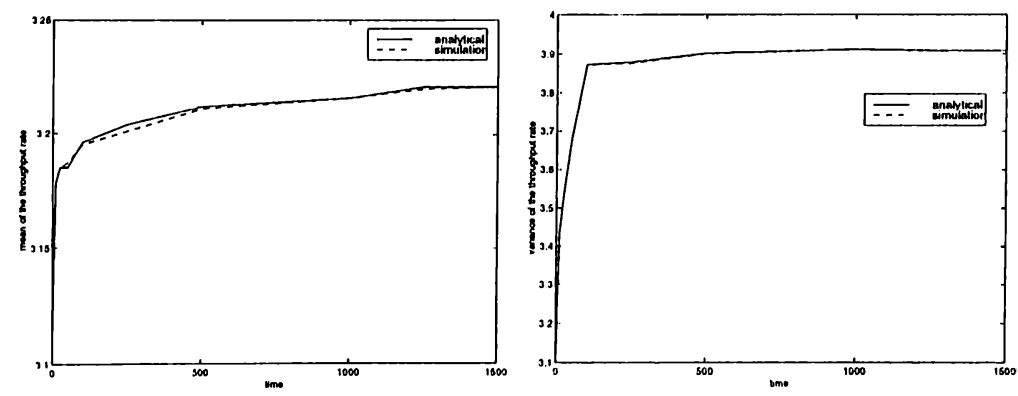

Figure 4.7: Mean and Variance vs time: the three-machine model

under consideration. The numerical results of the proposed analytical method for two-part type systems are obtained. The comparison of the analytical models with the the simulation models for the transient behavior and with the state-space representations for the long run behavior verify the accuracy of the methodology developed in this thesis. 


\section{Chapter 5}

\section{Extension, conclusion, and future research}

\subsection{Extension}

In this section, we briefly discuss certain issues requiring further investigations. Each issue can be interpreted as a future research direction on the work accomplished in this thesis.

\subsubsection{Line Behavior and Design Issues}

The line design problem is that of choosing buffer sizes or machine parameters to maximize performance or minimize cost subject to constraints. In order to solve such a problem, it is necessary to evaluate the performance measures of lines. The best performance measure of a transfer line implies the maximum throughput rate. The throughput rate is a nondecreasing function of the arrival rate of the parts, the machine parameters and the buffer capacities. This leads to the optimization problem of finding the arrival rate and the machine 
parameters and the buffer allocation with the restriction of achieving a desired throughput rate $E_{d}$ :

$$
\text { Find } \operatorname{cic}_{\substack{\mu_{1}, \ldots, \mu_{n} \\ b_{0} \ldots b_{n}}} E\left(\lambda, \mu_{1}, \ldots, \mu_{n}, b_{0}, \ldots, b_{n}\right) \text { such that } E \geq E_{d} .
$$

However, in the above statement, variability is ignored since there are essentially no methods for evaluating variability. However, this study enables us to analyze the system under consideration in terms of both the mean and the variance of the throughput rate. Meanwhile, we recommend to make use of the coefficient of variation rather than the variance since the former is more meaningful when comparing the variability of the random variables having different means. Table 5.1 presents an example in which the objective is to determine the size of the second buffer $\left(b_{1}=5\right.$ or $\left.b_{1}=20\right)$ while achieving a target throughput rate in a time interval of $(0,100]$.

\begin{tabular}{|c|c|c|c|c|}
\hline$\left(b_{1}, \lambda, \mu_{1}, \mu_{2}, \mu_{3}, t\right)$ & \multicolumn{2}{|c|}{$b_{2}=5$} & \multicolumn{2}{c|}{$b_{2}=20$} \\
\hline \hline & Mean & CV & Mean & CV \\
\hline$(2,20,6,4,3,2)$ & 2.012 & 0.580 & 1.733 & 0.627 \\
\hline$(2,20,6,4,3,5)$ & 2.296 & 0.532 & 2.260 & 0.564 \\
\hline$(2,20,6,4,3,10)$ & 2.511 & 0.514 & 2.591 & 0.557 \\
\hline$(2,20,6,4,3,25)$ & 2.674 & 0.486 & 2.815 & 0.542 \\
\hline$(2,20,6,4,3,50)$ & 2.703 & 0.484 & 2.898 & 0.574 \\
\hline$(2,20,6,4,3,100)$ & 2.713 & 0.485 & 2.947 & 0.589 \\
\hline$(2,20,6,4,3,200)$ & 2.714 & 0.488 & 2.946 & 0.591 \\
\hline
\end{tabular}

Table 5.1: Mean and Coefficient of Variation vs time: the two-machine model

The steady-state mean and variance of the throughput rate of the system with a larger buffer size is expected to be higher than that of the system with a smaller buffer size. In the above example, although the variance of the latter system (i.e., $b_{1}=20$ ) is larger at each instant in time due to its large buffer size, the mean throughput rate of the corresponding system is smaller in the time interval of $(0,5]$. This is mainly due to the assumption of empty and initial conditions. As the time from initialization becomes large, the parts start to accumulate in the buffer and the effect of the buffer size becomes apparent on the mean and variance of the throughput rate of the system under consideration and what is expected holds for the time starting from the instant of 10 time units (i.e., $E_{b_{1}=5}(t) \leq E_{b_{1}=20}(t)$ and $V_{b_{1}=5}(t) \leq V_{b_{1}=20}(t), \quad t>5$ ). 
Since it has been observed that the coefficient of variation for different alternatives either in the transient period or in the long run, achieves convexity, the following optimization problem can be utilized in order to minimize the variability of the throughput rate with the restriction of achieving a desired throughput rate $E_{d}$ :

Min $C V(t)$ such that $E(t) \geq E_{d}$.

In this problem, the mean and the variance of the throughput rate are the functions of time. By letting, in the limit, time approach infinity, the corresponding optimization problem can be written in terms of the steadystate performance measures of the system:

$$
\text { Min } C V \text { such that } E \geq E_{d} \text {. }
$$

However, there exists an advantage in making use of the second formulation rather than the last one. It brings the flexibility of optimizing a particular system for varying time instants. Meanwhile, these formulations enable us to determine the system with the minimum coefficient of variation of the throughput rate among different alternative systems achieving the desired mean throughput rate.

This way of interpretation might remove a deficiency in the transfer-line models in the literature-namely, that they do not accurately model the daily operation of the transfer line. Since the mean and the variance of the number . of units leaving the transfer line, are calculated, then an interval estimate for the actual production during a period of time that can be interpreted as a production shift, can be calculated. Such an interval estimate allows the manager to plan for shifts where output might be higher or lower than planned.

\subsubsection{Longer transfer lines}

Since the number of states of the Markov chains grows very fast with the number of machines, the buffer capacities, and the number of phases of the 
distributions, the tractable models in the transfer line literature are mostly of limited sizes. A similar statement applies to our case. As the number of machines and buffers in the system increase, the tractability of the evolution of the stochastic processes decreases by a considerable amount even if the arrival and service times are exponentially distributed. Therefore, it becomes harder to write down the corresponding representations as well as the codes in the Maple environment. So, decomposition techniques together with the proposed method should be utilized in order to obtain the distribution of the throughput of the longer lines.

\subsubsection{Non-exponential distributions}

The Markovian class includes systems in which the arrival and service processes can be represented as exponential, Erlangian, hyperexponential, and phasetype random variables. The general assumption in the transfer line literature is that the arrival and service processes are represented via the exponential distribution. It is argued that the distribution type that is the best in reflecting the real system behavior is the truncated normal distribution. Simultaneously, Knott and Sury [128] and Buzacott and Shantikumar [43] suggest that the real work stations exhibit positive skewness as does the lognormal distribution. Powell and Pyke [186] examine commonly used distributions (uniform, normal, and lognormal), and the beta distribution with both standard and unusual shapes. Their results indicate that the differences among different distributions increase with the complexity of the line and the coefficient of variation. The magnitude of the differences is around $2 \%$ for standard distributions, but can rise to $5 \%$ for distributions with identical first two moments and similar shapes. Distributions with non-standard shapes can give rise to differences many times larger than these. Therefore, the common assumption of exponential distribution turns out to be an. important deficiency in the transfer line literature.

In this research, the machines are considered to have mutually independent 
processing times that are exponentially distributed. Despite this assumption, the evolutions of the output processes of the corresponding systems also hold for the systems with non-exponential processing times. However, the closed-form expressions for the mean and variance of the throughput rate of the system of interest will differ from the available ones depending on the probability density and distribution functions of the relevant distributions that are readily convoluted via the convolution method (Rohatgi [196]) to obtain exact values of the mean and variance of the performance measure as explained in detail in Section 3.3.2.

In the graphical illustration of the stochastic processes under consideration, we make use of the memorylessness property of the inter-arrival times. In case of the distributions other than the exponential (or geometric) distribution to represent the arrival process, a method that keeps track of the arrival instants of the parts should be recommended. Our approach can then be utilized, however, such a method introduces a considerable amount of complexity in terms of the mathematical calculations. We believe that the advancement in the related software technology can help to alleviate such difficulties today. Therefore, this study can be extended to the systems with non-Poisson arrival process by relaxing the strict assumption of the exponential distribution.

\subsubsection{Pull-type systems}

In general, flow lines are push-type systems where little attention is given to the finished-product inventory. The line is to produce as much as it can with the assumption that all finished products are either used or demanded. Otherwise, it has to stop producing when there is an excessive accumulation of finished products. Those systems are known as pull-type systems.

The general philosophy of pull systems is to produce as much as needed, while that of push systems is to produce as much as possible. It is conceivable that pull systems may adapt to external changes faster than push systems and may have a smaller inventory accumulation. Therefore, the modeling of 
transfer lines as push-type systems rather than the pull-type systems turns out to be a deficiency in the transfer line literature.

We may assume an infinite supply of raw material for the facility to process. The finished products are stored in the warehouse until they are used to satisfy customer demand. Production may continue as long as the inventory level in the warehouse is below a target level. That is, it stops as soon as the inventory level reaches its target, and it restarts when the level drops below the target. This principle of operation is known as the base-stock policy in the classical inventory theory. It can be utilized by a control mechanism that would incorporate the proposed model and the solution technique. This would help to remove the corresponding deficiency in the transfer line literature.

\subsubsection{General networks}

Throughout the paper, we have been concerned with flow line models. The special structure of these models, ie., a series of machines separated by buffers, allows many results to be obtained. Although flow line structures are often encountered in industry, there also exist manufacturing systems exhibiting more general structures. Among these, assembly systems are of great interest and can be viewed as an extension of the flow line structure.

There has been relatively little work on Assembly/Disassembly networks compared with the vast queueing theory literature. This may be due to one of the earliest results: Harrison [92] found that assembly systems with infinite buffers are inherently unstable. That is, there is no steady-state probability distribution and that at least one buffer level increases without bounds. This result must have been discouraging to those who wished to study assembly systems by means of traditional queueing theory methods.

Actually, these systems can be reduced to a flow line structure in case of satisfying a set of assumptions : 
- The routing is entirely deterministic. That is, the path that each part follows in the system is known in advance and the contents of each buffer are homogeneous.

- The only source of randomness is the variation of processing times of the machines.

- They should be connected tree-structured networks in which exactly one sequence of machines and buffers connects any two machines in the network. If there are $n_{M}=n$ machines, then there are $n_{B}=n-1$ buffers. In fact, this is a generalization of a transfer line.

Then, the proposed method can calculate the mean and the variance of the throughput rate of these systems.

\subsection{Conclusion and future research}

In this thesis we propose an analytical method for estimating the performance measures of a serial production line with reliable machines and finite buffers. The transient and steady-state behaviors of this system are determined by using the evolution of the stochastic processes under consideration. The analysis of the evolution of stochastic processes enables us to derive the distribution of the throughput of the system. This distribution is then utilized to find the higher order moments of the throughput in measuring the performance of the system. The method based on the analytical derivation of the distribution provides good results for typical transfer line models encountered in real applications. Moreover, the iterative algorithms coded in Maple V environment seem to be efficient: in all examples we tested, it always converged and in general very rapidly. Furthermore, the analytical models are compared with the state-space representations and simulation models in which the results verified the accuracy of the methodology developed in this thesis.

In the literature, there is only Karpelevitch and Kreinin [115] who consider 
the joint stationary distribution of the waiting times of the part at the $j^{\text {th }}$ station and the joint stationary distribution of the number of parts at the arrival epoch. However, we examine the transient behavior of transfer lines up to three machines and then derive the distribution of the performance measures of interest. The major contribution of this work is that it makes use of the evolution of the stochastic processes in determining the higher order moments of the performance measures over a period of any length.

This research should be extended to develop analytical methods for the analysis of more complicated systems such as longer transfer lines with nonexponential distributions and multiple-part types. Although our models make a number of simplifying assumptions, the proposed method can be utilized to calculate the mean and variance of throughput of transfer lines with their mostly encountered assumptions in the literature as relaxed: The first buffer of the line is assumed to have zero capacity ((i.e., the parts arriving the system while the first machine is busy are lost). In case of determining the evolution of the output process from the first machine of the line, this assumption can be readily relaxed by generalizing the system behavior starting from a typical realization for a system with a finite capacity of the first buffer in the line. In this way, all the parts arriving the system when the first machine is busy are not necessarily lost. Similarly, the assumption of the infinite capacity of the last buffer in the line (i.e., last machine never gets blocked) can be relaxed by determining the evolution of the output process from the last machine of the line. Another simplifying assumption for the model of interest is the existence of empty and idle initial conditions. This assumption can be readily relaxed by shifting the evolution of the output process of the line up by the number of parts initially present. This leads to an increase of the initial number of parts in the system in the mean of the number of parts leaving the relevant system at an arbitrary instant in time while the variance of throughput rate of the current system is as the same as the one of the system with empty and idle initial conditions.

Furthermore, line design issues, pull-type systems, and general networks can be studied by making use of this method. 


\section{Bibliography}

[1] J. Abate and W. Whitt. Transient behavior of regulated brownian motion, i: Starting at the origin. Advan Appl Probab, 19:560-598, 1987.

[2] J. Abate and W. Whitt. Transient behavior of regulated brownian motion, ii: Non-zero initial conditions. Advan Appl Probab, 19:599-631, 1987.

[3] J. Abate and W. Whitt. Transient behavior of the $m / m / 1$ queue: Starting at the origin. Queueing Sys, 2:41-65, 1987.

[4] J. Abate and W. Whitt. Approximations for the $m / m / 1$ busy-period distribution. In J. W. Cohen, O. J. Boxma, and R. Syski, editors, Queueing Theory and Its Applications, North Holland, Amsterdam, 1988.

[5] J. Abate and W. Whitt. The correlation functions of $\mathrm{rbm}$ and $m / m / 1$. Stochastic Models, 4:315-359, 1988.

[6] J. Abate and W. Whitt. Simple spectral representations for $m / m / 1$ queue. Queueing Sys, 3:321-346, 1988.

[7] J. Abate and W. Whitt. Transient behavior of the $m / m / 1$ queue via laplace transforms. Advan Appl Probab, 20:145-178, 1988.

[8] J. Abate and W. Whitt. Transient behavior of the $\mathrm{m} / \mathrm{g} / 1$ workload process. Opns Res, 42:750-764, 1994.

[9] V. G. Adkalha and G. S. Fishman. Starting and stopping rules for simulations using a priori information. Eur J Opl Res, 10:379-394, 1982. 
[10] A. Alkaff and E. J. Muth. The throughput rate of multi-station production lines with stochastic servers. Probability in the Engineering and Informational Sciences, 1:309-326, 1987.

[11] T. Altiok. Performance Analysis of Manufacturing Systems. Springer Series in Operations Research, Springer-Verlag, New York, 1996.

[12] S. Andradóttir. Optimization of the transient and steady-state behavior of discrete-event systems. Mgmt Sci, 42:717-737, 1996.

[13] S. Asmussen. Applied Probability and Queues. Wiley, 1987.

[14] S. Asmussen. Queueing simulation in heavy traffic. Mgmt Sci, 17:84-111, 1992.

[15] S. Asmussen, P. W. Glynn, and H. Thorisson. Stationarity detection in the initial transient problem. ACM Trans on Modeling and Comp Simulation, 2:130-157, 1992.

[16] F. Baccelli and A. M. Makowski. Dynamic, transient and stationary behavior of the $m / g i / 1$ queue via martingales. Annals Probab, 14:1691$1699,1989$.

[17] F. Baccelli and A. M. Makowski. Exponential martingales for queues in a random environment: The $m / g / 1$ case. Technical report, Department of Electrical Engineering, University of Maryland, 1989.

[18] S. X. Bai and M. Elhafsi. Transient and steady-state analysis of a manufacturing system with setup changes. $J$ of Global Opt, 8:349-378, 1996.

[19] N. T. Bailey. The Elements of Stochastic Processes with Applications to the Natural Sciences. Wiley, 1964.

[20] N. T. J. Bailey. A continuous time treatment of a simple queue using generating functions. J R Statist Soc (Series B), 16:288-291, 1954.

[21] N. T. J. Bailey. Some further results in the non-equilibrium theory of a simple queue. J R Statist Soc (Series B), 19:326-333, 1957. 
[22] Z. Barzily and D. Gross. Transient solutions for repairable item provisioning. Technical report, The George Washington University, 1979.

[23] V. Benes̃. On queues with poisson arrivals. Annals Math Statist, 28:670$677,1957$.

[24] V. Benes̃. General Stochastic Processes in the Theory of Queues. Addison-Wesley, 1963.

[25] D. J. Bertsimas, J. Keilson, D. Nakazato, and H. Zhang. Transient and busy period analysis of the $g i / g / 1$ queue: Solution as a hilbert problem. J Appl Probab, 10:153-184, 1990.

[26] D. J. Bertsimas, J. Keilson, D. Nakazato, and H. Zhang. Transient and busy period analysis of the $g i / g / 1$ queue: As a hilbert problem. $J$ Appl Probab, 28:873-885, 1991.

[27] D. J. Bertsimas and D. Nakazato. Transient and busy period analysis of the $g i / g / 1$ queue: The method of stages. Queueing Sys, 10:153-184, 1992.

[28] U. N. Bhat and I. Sahin. Transient behavior of queueing systems. $m / d / 1, m / e_{k} / 1, d / m / 1$, and $e_{k} / m / 1$. Technical report, Department of Operations Research, Case Western Reserve University, 1969.

[29] D. P. Bischak. Weighted Batch Means for Improved Confidence Intervals for Steady-State Processes. PhD thesis, Department of Industrial and Operations Engineering, University of Michigan, 1988.

[30] J. P. C. Blanc and E. A. Van Doorn. Relaxation times for queueing systems. In J. W. De Bakker et al, editor, Mathematics and Computer Science, Proceedings of the CWI Symposium, North-Holland, Amsterdam, 1983.

[31] W. Bohm. Markovian queueing systems in discrete time. Maths Sys in Econom, 137, 1993.

[32] W. Bohm and S. G. Mohanty. The transient analysis of $m / m / 1$ queues in discrete time with general server vacations. I Appl Probab, 1992. 
[33] W. Bohm and S. G. Mohanty. The transient solution of $m / m / 1$ queues under $(m, n)$-policy: A combinatorial approach. J Statist Planning Inference, 34:23-33, 1993.

[34] W. Bohm and S. G. Mohanty. On discrete time markovian n-policy queues involving batches. Sankya, Series A, 56:1-20, 1994.

[35] W. Bohm and S. G. Mohanty. Transient analysis of queues with heterogeneous arrivals. Queueing Sys, 18:27-45, 1994.

[36] S. Browne and J. M. Steele. Transient behavior of coverage processes with applications to the infinite-server queue. $J$ Appl Probab, 30:589$602,1993$.

[37] H. Bruneel. Analysis of buffer behavior for an integrated voice-data system. Electron Letters, 19:72-74, 1983.

[38] H. Bruneel. On the behavior of buffers with random server interruptions. Performance Evaluation, 3:165-175, 1983.

[39] H. Bruneel. Analysis of an infinite buffer system with random server interruptions. Comp Opns Res, 11:373-386, 1984.

[40] H. Bruneel. Analysis of discrete-time buffers with one single output channel, subjected to a general interruption process. In Proc. $10^{\text {th }}$ International Symposium on Computer Performance, pages 103-115, Paris, France, 1984.

[41] H. Bruneel. Exact derivation of transient behavior for buffers with random output interruptions. Comp Networks and ISDN Sys, 22:277$285,1991$.

[42] J. A. Buzacott and J. G. Shanthikumar. Design of manufacturing systems using queueing models. Queueing Sys, 12:135-214, 1992.

[43] J. A. Buzacott and J. G. Shanthikumar. Stochastic Models of Manufacturing Systems. Prentice-Hall, Inc, New York, 1993. 
[44] S. S. L. Chang. Simulation of transient and time-varying conditions in queueing network. In Proceedings of the Seventh Annual Pittsburgh Conference on Modeling and Simulation, pages 1075-1078, University of Pittsburgh, 1977.

[45] Y. F. Choong and S. B. Gershwin. A decomposition method for the approximate evaluation of capacitated transfer lines with unreliable machines and random processing times. IIE Trans, 19:150-159, 1987.

[46] G. M. Clark. Use of polya distributions in approximate solutions to non-stationary $\mathrm{m} / \mathrm{m} / \mathrm{s}$ queues. Commun ACM, 24:206-217, 1981.

[47] J. W. Cohen. The Single Server Queue, volume 2. Wiley, 1969.

[48] T. Collings and C. Stoneman. The $m / m / \infty$ queue with varying arrival and departure rates. Opns Res, 24:760-773, 1976.

[49] B. W. Conolly and C. Langaris. On a new formula for the transient state probabilities for $m / m / 1$ queues and computational implications. $J$ Appl Probab, 30:237-246, 1993.

[50] M. A. Crane and D. L. Iglehart. Simulating stable stochastic systems, i: General multi-server queues. J Assoc Comp Mach, 21:103-113, 1974.

[51] M. A. Crane and D. L. Iglehart. Simulating stable stochastic systems, ii: Markov chains. J Assoc Comp Mach, 21:114-123, 1974.

[52] M. A. Crane and D. L. Iglehart. Simulating stable stochastic systems, iii: Regenerative processes and discrete-event simulation. Opns Res, 23:33$45,1975$.

[53] M. A. Crane and A. J. Lemoine. An Introduction to the Regenerative Method for Simulation Analysis, Lecture Notes in Control and Information Sciences, volume 4. Springer-Verlag, 1977.

[54] A. Csenki. The joint distribution of sojourn times in finite markov processes. Advan Appl Probab, 24:141-160, 1992. 
[55] J. N. Daigle and M. N. Magalhães. Transient behavior of $m / m^{i j} / 1$ queues. Queueing Sys, 8:357-378, 1991.

[56] Y. Dallery and S. B. Gershwin. Manufacturing flow line systems: A review of models and analytical results. Queueing Sys, 12:3-94, 1992.

[57] B. Deler and C. Dinçer. On the behavior of throughput of transfer lines. Technical report, Industrial Engineering Department, Bilkent University, 1998.

[58] B. Deler and I. Sabuncuğlu. An analysis of factors affecting transient state of non-terminating systems. Technical report, Department fo Industrial Engineering, Bilkent University, 1997.

[59] C. Dinçer and N. Donmez. Analysis of erlang transfer lines. Working Paper. Bilkent University, Ankara, 1997.

[60] R. R. Duersch and L. W. Schruben. An interactive run length control for simulations on pcs. In Proceedings of Winter Simulation Conference, pages 866-870, Washington, D.C., 1986.

[61] W. Feller. An Introduction to Probability Theory and its Applications, volume 1. Wiley, 1968.

[62] G. S. Fishman. Spectral Methods in Econometrics. Harvard University Press, 1969.

[63] G. S. Fishman. Estimating sample size in computer simulation experiments. Mgmt Sci, 18:21-38, 1971.

[64] G. S. Fishman. Concepts and Methods in Discrete Event Digital Simulation. Wiley, 1973.

[65] G. S. Fishman. Statistical analysis for queueing simulations. Mgmt Sci, 20:363-369, 1973.

[66] G. S. Fishman. Estimation in multi-server queueing simulations. Opns Res, 22:72-78, 1974. 
[67] G. S. Fishman. Achieving specific accuracy in simulation output analysis. Commun Assoc Comp Mach, 20:310-315, 1977.

[68] G. S. Fishman. Principles of Discrete Event Simulation. Wiley, 1978.

[69] Y. Frein, C. Commault, and Y. Dallery. Modeling and analysis of closedloop production lines with unreliable machines and finite buffers. IIE Trans, 28:545-554, 1996.

[70] A. V. Gafarian, J. Ancker, and C. J. Morisaku. Evaluation of commonly used rules for detecting steady-state in computer simulation. Nav Res $\log Q, 25: 511-529,1978$.

[71] P. L. Gall. The overall sojourn time in tandem queues with identical successive service times and renewal input. Stochastic Processes and their Applications, 52:165-178, 1994.

[72] M. A. Gallagher, J. Bauer, and P. S. Maybeck. Initial data truncation for univariate output of discrete-event simulations using the kalman filter. Mgmt Sci, 42:559-575, 1996.

[73] S. B. Gershwin. An efficient decomposition method for the approximate evaluation of tandem queues with finite storage space and blocking. Opns Res, 35:291-305, 1987.

[74] S. B. Gershwin. Manufacturing Systems Engineering. Prentice-Hall, New Jersey, 1994.

[75] C. R. Glassey and Y. Hong. Analysis of behavior of an unreliable $n$ stage transfer line with $(n-1)$ inter-stage storage buffers. Int J Prod Res, 31:519-530, 1993.

[76] P. W. Glynn. Some new results on the initial transient problem. In Proceedings of the 1995 Winter Simulation Conference, pages 165-170, 1995.

[77] P. W. Glynn and P. Heidelberger. Analysis of initial transient deletion for replicated steady-state simulations. Opns Res Letters, 10:437-443, 1991. 
[78] P. W. Glynn and P. Heidelberger. Analysis of initial transient deletion for parallel steady-state simulations. Siam J Sci Statist Comp, 13:904-922, 1992.

[79] P. W. Glynn and P. Heidelberger. Experiments with initial transient deletion for parallel, replicated steady-state simulations. Mgmt Sci, 38:400-418, 1992.

[80] P. W. Glynn and D. L. Iglehart. The theory of standardized time series. Mathematics Opns Res, 15:1-16, 1992.

[81] D. Goldsman and M. S. Meketon. A comparison of several variance estimators. Technical report, School of Industrial and Systems Engineering, Georgia Institute of Technology, 1990.

[82] D. Goldsman and L. W. Schruben. Asymptotic properties of some confidence interval estimators for simulation output. Mgmt Sci, 30:1217$1225,1984$.

[83] D. Goldsman and L. W. Schruben. New confidence interval estimators using standardized time series. Mgmt Sci, 36:393-397, 1990.

[84] M. N. Gopalan and U. Dinesh Kumar. Stochastic analysis of a two-stage production system with $n$ parallel stations in the first stage. Int $J$ Mgmt Sys, 8:263-275, 1992.

[85] M. N. Gopalan and U. Dinesh Kumar. On the transient behavior of a merge production system with an end buffer. Int $J$ Prod Econom, 34:157-165, 1994.

[86] W. K. Grassman. Transient solutions in markovian queueing systems. Comp Opns Res, 4:47-53, 1977.

[87] W. K. Grassman. Transient and steady-state results for two parallel queues. Omega, 8:105-112, 1980.

[88] W. K. Grassman and J. Servranckx. The que package. mimeographed, 1979. University of Saskatchewan, Saskatoon, Canada. 
[89] D. Gross and C. Harris. Fundamentals of Queueing Theory. Wiley, 1985.

[90] F. M. Guillemin, R. R. Mazumdar RR, and A. D. Simonian. On heavy traffic approximations for transient characteristics of $m / m / \infty$ queues. $J$ Appl Probab, 33:490-506, 1996.

[91] S. Halfin and W. Whitt. Heavy-traffic limits for queues with many exponential servers. Opns Res, 29:567-588, 1981.

[92] J. M. Harrison. Assembly-like queues. J of Appl Probab, 10:354-367, 1973.

[93] J. M. Harrison. Brownian Motion and Stochastic Flow Systems. Wiley, 1977.

[94] C. R. Heathcote. Complete exponential convergence and some related topics. J Appl Probab, 4:217-256, 1967.

[95] C. R. Heathcote and P. Winer. $\Lambda \mathrm{n}$ approximation for the moments of waiting times. Opns Res, 17:175-186, 1969.

[96] J. M. Heavey, H. T. Papadopoulos, and J. Browne. The throughput rate of multi-station unreliable production lines. Eur J of Opl Res, 68:69-89, 1993.

[97] P. Heidelberger and P. D. Welch. Adaptive spectral methods for simulation output analysis. IBM J Res Develop, 25:860-876, 1981.

[98] P. Heidelberger and P. D. Welch. A spectral method for confidence interval generation and run length control in simulations. Commun Assoc Comp Mach, 24:233-245, 1981.

[99] P. Heidelberger and P. D. Welch. Simulation run length control in the presence of an initial transient. Opns Res, 31:1109-1114, 1983.

[100] K. B. Hendricks. The output processes of serial production lines of exponential machines with finite buffers. Opns Res, 40:1139-1147, 1992. 
[101] K. B. Hendricks and J. O. McClain. The output processes of serial production lines of general machines with finite buffers. Mgmt Sci, 39:1194-1201, 1993.

[102] D. P. Heyman. An approximation for the busy period of the $\mathrm{m} / \mathrm{g} / \mathrm{l}$ queue using a diffusion model. J Appl Probab, 11:159-169, 1974.

[103] D. P. Heyman and M. J. Sobel. Stochastic Models in Operations Research, volume 1. McGraw-Hill, 1985.

[104] Y. Hong, C. R. Glassey, and D. Seong. The analysis of a production line with unreliable machines and random processing times. IIE Trans, 24:77-83, 1992.

[105] L. Horváth. Strong approximations of open queueing networks. Mathematics of Opns Res, 17:487-508, 1992.

[106] J. R. Jackson. Networks of waiting lines. Opns Res, 5:518-521, 1957.

[107] S. H. Jacobson. The effect of initial transient on the steady-state simulation harmonic analysis gradient estimators. Maths and Comp in Simulation, 43:209-221, 1997.

[108] R. Jain and I. Chlamtac. The $p^{2}$ algorithm for dynamic calculation of quantiles and histograms without storing observations. Commun Assoc Comp Mach, 28:1076-1085, 1985.

[109] A. Jean-Marie and F. Robert. On the transient behavior of the processor sharing queue. Queueing Sys, 17:129-136, 1994.

[110] Y. C. Jenq. On calculations of transient statistics of a discrete queueing system with independent general arrivals and geometric departures. IEEE Trans Commun, 28:908-910, 1980.

[111] S. Kanwar and J. L. Jain. Combinatorial approach to markovian queues. J Statist Planning Inference, 34:55-67, 1993. 
[112] S. Kanwar, J. L. Jain, and J. M. Gupta. Lattice path approach to transient solution of $m / m / 1$ with $(0, k)$ control policy. J Statist Planning Inference, 34:68-82, 1993.

[113] S. Karlin and L. McGregor. The differential equations of birth-and-death processes and the stieltjes moment problem. Trans Amer Maths Soc, 85:489-546, 1957.

[114] F. I. Karpelevitch and A. Y. Kreinin. Joint distributions in poissonian tandem queues. Queueing Sys, 12:273-286, 1992.

[115] F. I. Karpelevitch and A. Y. Kreinin. Joint distributions in poissonian tandem queues. Queueing Sys, 12:273-286, 1992.

[116] J. Keilson. Markov Chain Models-Rarity and Exponentiality. SpringerVerlag, 1979.

[117] J. Keilson and A. Kooharian. On time-dependent queueing processes. Maths Statist, 31:104-112, 1960.

[118] W. D. Kelton. The startup problem in discrete event simulation. Technical report, Department of Industrial Engineering, University of Wisconsin, 1980.

[119] W. D. Kelton and A. M. Law. A new approach for dealing with the startup problem in discrete event simulation. Nav Res Log $Q, 30: 641-$ $658,1983$.

[120] W. D. Kelton and A. M. Law. The transient behavior of the $\mathrm{m} / \mathrm{m} / \mathrm{s}$ queue with implications for steady-state simulation. Opns Res, 33:378$396,1985$.

[121] D. G. Kendall. Some problems in the theory of queues. J R Statist Soc (Series B), 13:151-185, 1951 .

[122] D. G. Kendall. Stochastic processes occurring in the theory of queues and their analysis by the method of the embedded markov chain. Annals of Maths and Statist, 24:338-354, 1953. 
[123] D. G. Kendall. Geometric ergodicity and the theory of queues. In K. J. Arrow, S. Karlin, and P. Suppes, editors, Mathematical Method in the Social Sciences. Stanford University Press, 1960.

[124] J. F. C. Kingman. Regenerative Phenomena. Wiley, 1972.

[125] L. Kleinrock. Queueing Systems, Vol. I: Theory. Wiley, 1975.

[126] G. A. Klutke and M. Seiford. Transient behavior of finite capacity tandem queues with blocking. Int J Sys Sci, 22:2205-2215, 1991.

[127] C. Knessl. On the transient behavior of the erlang loss model. Commun Statist Stochastic Models, 6:749-776, 1990.

[128] K. Knott and R. J. Sury. A study of work-time distributions on unpaced tasks. IIE Trans, 19:50-55, 1987.

[129] H. Kobayashi and Q. Ren. A mathematical theory for transient analysis of communication networks, 1992. Special Issue on Teletraffic: the IEICE Trans Comm.

[130] H. Kobayashi and Q. Ren. Non-stationary behavior of statistical multiplexing for multiple types of traffic. In Proc. $26^{\text {th }}$ Annual Conf. on Information Sciences and Systems, Princeton University, 1992.

[131] H. Kobayashi and Q. Ren. Transient solutions for the buffer behavior in statistical multiplexing. Performance Evaluation, 23:65-87, 1995.

[132] B. O. Koopman. Air-terminal queues under time-dependent conditions. Opns Res, 20:1089-1114, 1972.

[133] T. C. T Kotiah. Approximate transient analysis of some queueing systems. Opns Res, 26:333-346, 1978.

[134] S. S. Lavenverg. The steady-state queueing-time distribution for the m/g/1 finite capacity queue. Mgmt Sci, 21:501-506, 1975.

[135] A. M. Law. Efficient estimators for simulated queueing systems. Technical report, Operations Research Center, University of California, Berkeley, 1974. 
[136] A. M. Law. Statistical analysis of simulation output data. Opns Res, 31:983-1029, 1983.

[137] A. M. Law and J. S. Carson. A sequential procedure for determining the length of a steady-state simulation. Opns Res, 27:1011-1025, 1979.

[138] A. M. Law and W. D. Kelton. Confidence intervals for steady-state simulations, ii: A survey of sequential procedures. Mgmt Sci, 28:550$562,1982$.

[139] A. M. Law and W. D. Kelton. Confidence intervals for steady-state simulations, i: A survey of fixed sample size procedures. Opns Res, 32:1221-1239, 1984.

[140] A. M. Law and W. D. Kelton. Simulation Modeling and Analysis. McGraw Hill, Singapore, 1991.

[141] D. Lee and S. Li. Transient analysis of multi-server queues with markov-modulated poisson arrivals and overload control. Performance Evaluation, 16:49-66, 1992.

[142] D. Lee and S. Li. Transient analysis of a switched poisson arrival queue under overload control. Performance Evaluation, 17:13-29, 1993.

[143] D. Lee and E. Roth. A heuristic for the transient expected queue length of markovian queueing systems. Opns Res Letters, 14:25-27, 1993.

[144] D. S. Lee. Transient Queueing Analysis and Performance of Packet Video Systems. PhD thesis, Department of Electrical Engineering, Columbia University, 1989.

[145] I. J. Lee. Stationary markovian queueing systems: An approximation for the transient expected queue length. Master's thesis, Massachusetts Institute of Technology, 1985.

[146] L. Lin and J. K. Cochran. Metamodels of production line transient behavior for sudden machine breakdowns. Int $J$ Prod Res, 28:1791-1806, 1990. 
[147] G. Louchard. Large finite population queueing systems. the single-server model. Stochastic Processes and their Applications, 53:117-145, 1994.

[148] F. Machihara. Transition probabilities of markovian service system and their application. Rev Elect Commun Labs, 29:170-188, 1981.

[149] M. N. Magalhães and R. L. Disney. Departures from queues with changeover times. Queueing Sys, 5:295-312, 1989.

[150] N. B. Marks. A study of times to reach equilibrium in an $\mathrm{m} / \mathrm{m} / \mathrm{s}$ queueing system. Technical report, University of Miami, 1979.

[151] W. A. Massey. An operator analytic approach to the jackson network. $J$ Appl Probab, 21:379-39.3, 1984.

[152] W. A. Massey. A family of bounds for the transient behavior of a jackson network. J Appl Probab, 23:543-549, 1986.

[153] M. S. Meketon and B. W. Schmeiser. Overlapping batch means: Something for nothing? In Proceedings of the Winter Simulation Conference, pages 227-230, Dallas, 1984.

[154] M. R. Middleton. Transient effects in $M / G / 1$ queues. $\mathrm{PhD}$ thesis, Stanford University, 1979.

[155] G. J. Miltenburg. Variance of the number of units produced on a transfer line with buffer inventories during a period of length $t$. Naval Res Logist, 34:811-822, 1987.

[156] D. Mitra and A. Weiss. The transient behavior in erlang's model for large trunk groups and various traffic conditions. In Proceedings of the Twelfth International Teletraffic Congress, pages 5.1B.4.1--5.1B4.8, Torino, Italy, 1988.

[157] T Moeller and H Kobayashi. Use of diffusion approximation to estimate run length in simulation experiments. In G Bruckmann, F Fershel, and L Schmetterer, editors, Proceedings of Computational Statistics, pages 363-372, Vienna, 1974. Physica-Verlag. 
[158] D. C. Montgomery. Design and Analysis of Experiments. Wiley, New York, 1991.

[159] S. C. Moore. Approximating the behavior of non-stationary single-server queues. Opns Res, 23:1011-1032, 1975.

[160] M. Mori. Transient behavior of the mean waiting time and its exact forms in $m / m / 1$ and $m / d / 1$. Opns Res Soc Jpn, 19:14-31, 1976.

[161] T. Morisaku. Techniques for Data-Truncation in Digital Computer Simulation. PhD thesis, Department of Industrial and Systems Engineering, University of Southern California, 1976.

[162] T. D. Morris and H.G. Perros. Approximate analysis of a discrete-time tandem network of cut-through queues with blocking and bursty traffic. Performance Evaluation, 17:207-223, 1993.

[163] P. M. Morse. Stochastic properties of waiting lines. J Opns Res Soc Am, $3: 255-261,1955$.

[164] P. M. Morse. Queues, Inventories and Maintenance. Wiley, 1958.

[165] J. R. Murray and W. D. Kelton. The transient behavior of the $m / e_{k} / 2$ queue and steady-state simulation. Comp Opns Res, 15:357-367, 1988.

[166] E. J. Muth. Stochastic processes and their network representations associated with production line queueing model. Eur $J$ Opl Res, 15:63$83,1984$.

[167] Y. Narahari, N. Hemachondra, and M. S. Gaur. Transient analysis of multi-class manufacturing systems with priority scheduling. Comput and Opns Res, 24:387-398, 1997.

[168] Y. Narahari and N. Viswanadham. Transient analysis of manufacturing systems performance. IEEE Trans on Robotics and Automation, 10:230$244,1994$.

[169] M. F. Neuts. Structured stochastic matrices of $\mathrm{m} / \mathrm{g} / 1$ type and their applications. Marcel Dekker. 
[170] M. F. Neuts. The single-server queue in discrete time numerical analysis i. Nav Res Log Q, 20:297-304, 1973.

[171] G. F. Newel. Applications of Queueing Theory. Chapman and Hall, 1971.

[172] A. R. Odoni and E. Roth. An empirical investigation of the transient behavior of stationary queueing systems. J Opns Res Soc Am, 31:433$455,1983$.

[173] T. J. Ott. The covariance function of the virtual waiting time process in an $m / g / 1$ queue. Advan Appl Probab, 9:158-168, 1977.

[174] T. J. Ott. The stable $m / g / 1$ queue in heavy traffic and its covariance function. Advan Appl Probab, 9:169-186, 1977.

[175] J. Ou and S. B. Gershwin. The variance of the lead time distribution of a two-machine transfer line with a finite buffer. Technical report, MIT Laboratory for Manufacturing and Productivity, 1989.

[176] H. T. Papadopoulos. The throughput of multi-station production lines with no intermediate buffers. Opns Res, 43:712-715, 1993.

[177] H. T. Papadopoulos. An analytic formula for the mean throughput of $n$-station production lines with no intermediate buffers. Technical report, University of the Aegean, Department of Mathematics, 1994.

[178] H. T. Papadopoulos. The throughput rate of multi-station reliable production lines with no intermediate buffers. Opns Res, 4:712-715, 1995.

[179] H. T. Papadopoulos. An analytic formula for the mean throughput of n-station production lines with no intermediate buffers. Eur $J$ of $O p l$ Res, 91:481-494, 1996.

[180] H. T. Papadopoulos and J. M. Heavey, Queueing theory in manufacturing systems analysis and design: A classification of models for production and transfer lines. Eur J Opl Res, 92:1-27, 1996. 
[181] H. T. Papadopoulos and M. E. J. O'Kelly. Exact analysis of production lines with no intermediate buffers. Eur $J$ of Opl Res, 65:118-137, 1993.

[182] P. R. Parthasarathy. A transient solution to a $m / m / 1$ queue: A simple approach. Advan Appl Probab, 19:997--998, 1987.

[183] P. R. Parthasarathy and M. Sharafali. Transient solution to the manyserver poisson queue: A simple approach. J Appl Probab, 26:584-594, 1989.

[184] C. D. Pegden and M. Rosenshine. Some new results for the $m / m / 1$ queue. Mgmt Sci, 28:821-828, 1982.

[185] C. D. Pegden, R. E. Shannon, and R. P. Sadowski. Introduction to Simulation using SIMAN. McGraw-Hill, New York, 1996.

[186] S. G. Powell and D. F. Pyke. An empirical investigation of the twomoment approximation for production lines. Int J Prod Res, 32:1137$1157,1994$.

[187] N. U. Prabhu. Queues and Inventories. Wiley, 1965.

[188] N. U. Prabhu. Stochastic Storage Processes. Springer-Verlag, 1980.

[189] K. E. E. Raatikainen. Sequential procedure for simultaneous estimation of several percentiles. Technical report, Department of Computer Science, University of Helsinki, Helsinki, Finland, 1988.

[190] K. E. E. Raatikainen. Run length control using parallel spectral method. In Proceedings of the 1984 Winter Simulation Conference, pages 594-602, 1992.

[191] V. Ramaswami. The busy period of queues which have a matrixgeometric steady-state probability vector. Opsearch, 19:238-261, 1982.

[192] K. L. Rider. A simple approximation to the average queue size in the time-dependent $m / m / 1$ queue. J Assoc Comp Mach, 23:361-367, 1976.

[193] J. Riordan. Stochastic Service Systems. Wiley, 1988. 
[194] R. D. Ripley. Simulation methodology - an introduction for queueing theorists. Queueing Sys, 3:201-220, 1988.

[195] S. Robinson. An heuristic technique for selecting the run length of nonterminating steady-state simulations. Simulation, 65:170-179, 1995.

[196] V. K. Rohatgi. An Introduction to Probability Theory and Mathematical Statistics. Wiley, New York, 1976.

[197] W. A. Rosenkrantz. Calculation of the laplace transform of the length of the busy period for the $m / g i / 1$ queue via martingales. Annals Probab, $11: 817-818,1983$.

[198] E. Roth. A heuristic technique for the transient behavior of markovian queueing systems. Opns Res Letters, 3:301-305, 1985.

[199] E. Roth. The relaxation time heuristic for the initial transient problem in $m / m / k$ queueing systems. Eur $J$ Opl Res, 72:376-386, 1994.

[200] E. Roth and N. Josephy. The relaxation time heuristic for exponentialerlang queueing systems. Comput and Opns Res, 24:1761-1786, 1991.

[201] E. Roth and N. Josephy. The relaxation time heuristic for exponentialerlang queueing systems. Eur $J$ Opl Res, 72:376-386, 1993.

[202] E. Roth and A. H. Rutan. A relaxation time approach for reducing initialization bias in simulation. In Proceedings of the $18^{\text {th }}$ Annual Simulation Symposium, pages 189-203, Tampa, FL, 1985.

[203] M. H. Rothkopf and S. S. Oren. A closure approximation for the nonstationary $m / m / s$ queue. Mgmt Sci, 25:522-534, 1979.

[204] T. L. Saaty. Time-dependent solution of the many-server poisson queue. Opns Res, 8:755-772, 1960.

[205] H. Saito and F. Machihara. Transient analysis of a markovian loss system with heterogeneous inputs for time congestion measurement. Queueing Sys, 8:81-96, 1991. 
[206] R. G. Sargent, K. Kang, and D. Goldsman. An investigation of small sample size behavior of confidence interval estimation procedures. Technical report, Department of Industrial Engineering and Operations Research, Syracuse University, 1987.

[207] B. W. Schmeiser. Batch size effects in the analysis of simulation output. Opns Res, 30:556-568, 1982.

[208] F. A. V. D. Duyn Schoute and P. Wartenhorst. Transient analysis of a two-unit standby system with markovian degrading units. Mgmt Sci, 40:418-428, 1994.

[209] T. J. Schriber and R. W. Andrews. An arma-based confidence interval for the analysis of simulation output. Am J Math Mgmt Sci, 4:345-373, 1984.

[210] L. W. Schruben. Control of initialization bias in multi-variate simulation response. Commun ACM, 24:246-252, 1981.

[211] L. W. Schruben. Detecting initialization bias in simulation output. Opns Res, 30:569-590, 1982.

[212] L. W. Schruben. Confidence interval estimation using standardized time series. Opns Res, 31:1090-1108, 1983.

[213] L. W. Schruben, H. Singh, and L. Tierney. Optimal tests for initialization bias in simulation output. Opns Res, 31:1167-1178, 1983.

[214] Second International Workshop. Variance of Output of a Tandem Production System, North Carolina, USA, 1992.

[215] R. F. Serfozo, W. Szczotka, , and K. Topolski. Relating the waiting time in a heavy-traffic queueing system to the queue length. Stochastic Processes and their Applications, 52:119-134, 1994.

[216] O. P. Sharma. Markovian Queues. Ellis Horwood, Chichester, 1990.

[217] O. P. Sharma, N. Ravichandran, and J. Dass. Transient analysis of multiple-unit reliability systems. Nav Res Log Q, 36:255-264, 1989. 
[218] M. Snell and L. Schruben. Weighting simulation data to reduce initialization effects. IIE Trans, 17:354-363, 1985.

[219] K. Sohraby and J. Zhang. Transient analysis of discrete time queues with bursty and correlated arrivals arising in atm networks. In Proc. Conf. on Information Sciences and Systems, the John Hopkins University, Baltimore, MD, 1990.

[220] K. Sohraby and J. Zhang. Spectral decomposition approach for transient analysis of multi-server discrete-time queues. Performance Evaluation, 21:131-150, 1994.

[221] M. C. Springer. A decomposition approximation for finite-buffered flow lines of exponential queues. Eur $J$ of Opl Res, 74:95-110, 1994.

[222] D. A. Stanford, B. Pagurek, and C. M. Woodside. Optimal prediction of times and queue lengths in the $g i / m / 1$ queue. Opns Res, 31:322-337, 1983.

[22:3] L. Takács. Investigation of waiting time problems by reduction to markov processes. Acta Maths Acad Sci, 6:101-129, 1955.

[224] L. Takács. Introduction to the Theory of Queues. Oxford University Press, 1962.

[225] L. Takács. A single server queue with poisson input. Opns Res, 10:388-394, 1962.

[226] L. Takács. Combinatorial Methods in the Theory of Stochastic Processes. Wiley, 1967.

[227] B. Tan. Variance of the throughput of a n-station production line with no intermediate buffers and time-dependent failures. Technical report, Graduate School of Business, Koc University, 1996.

[228] B. Tan and S. Yeralan. A general decomposition method for heterogeneous multi-station production lines. Technical report, Koc University,Istanbul, 1994. 
[229] X. Tan and C. Knessl. Integral representations and asymptotics for infinite- and finite-capacity queues described by the unfinished work. Siam J Appl Math, 57:791-823, 1997.

[230] D. Towsley. An application of the reflection principle to the transient analysis of the $m / m / 1$ queue. Nav Res Log, 34:451-456, 1987.

[231] K. S. Trivedi. Probability and Statistics with Reliability, Queueing and Computer Science Applications. Prentice Hall, Inc., Englewood Cliffs, NJ, 1982.

[232] P. Tsoucas and J. Walrand. A note on stochastic bounds for queueing networks. Advan Appl Probab, 16:926-928, 1984.

[233] D. Vere-Jones. A rate of convergence problem in the theory of queues. Theory Probab Appl, 9:96-103, 1964.

[234] N. Viswanadham and Y. Narahari. Performance Modeling of Automated Manufacturing Systems. Englewood Cliffs, Prentice Hall, New Jersey, 1992.

[235] A. D. Wall and D. J. Worthington. Using discrete distributions to approximate general service time distributions in queueing models. $J$ Opl Res Soc, 45:1398-1404, 1994.

[236] P. D. Welch. On the problem of the initial transient in steady-state simulation. Technical report, IBM Watson Research Center, Yorktown Heights, New York, 1981.

[237] P. D. Welch. The statistical analysis of simulation results. In S. S. Lavenberg, editor, The Computer Performance Modeling Handbook, New York, 1983. Academic Press.

[238] W. Whitt. Comparing counting processes and queues. Advan Appl Probab, 13:207-220, 1981.

[239] W. Whitt. Planning queueing simulations. Mgmt Sci, 35:1341-1366, 1989. 
[240] W. Whitt. Simulation run length planning. In Proceedings of the 1989 Winter Simulation Conference, pages 106-112, 1989.

[241] W. Whitt. The efficiency of one long run versus independent replications in steady-state simulation. Mgmt Sci, 37:645-666, 1991.

[242] W. Whitt. Asymptotic formulas for markov processes with applications to simulation. Opns Res, 40:279-291, 1992.

[243] J. R. Wilson and A. A. B. Pritsker. Evaluation of startup policies in simulation experiments. Simulation, 31:79-89, 1978.

[244] J. R. Wilson and A. A. B. Pritsker. A survey of research on the simulation startup problem. Simulation, 31:55-58, 1978.

[245] Q. Wu. Variance of output of transfer lines with finite buffer inventories. Technical report, Department of Operations Research, Stanford University, 1994.

[246] S. Xie and C. Knessl. On the transient behavior of $m / m / 1$ and $m / m / 1 / k$ queues. Stud in Appl Math, 88:19l-240, 1993.

[247] S. Xie and C. Knessl. On the transient behavior of the erlang loss model: Heavy usage asymptotics. Siam J Appl Math, 53:555-599, 1993.

[248] M. N. Yunus. Transient blocking probability in a loss system with arbitrary initial conditions. J Maths Analysis and Appl, 147:397-402, 1990.

[249] J. Zhang and E.J. Coyle. Matrix recursive solutions for the transient of qbd-processes and its application to random access networks. In Proc. $22^{\text {nd }}$ Annual Conference on Information Sciences and Systems, Princeton University, New Jersey, 1988.

[250] J. Zhang and E.J. Coyle. Transient analysis of quasi-birth-death processes. Stochastic Models, 5, 1988. 


\section{Appendix A}

\section{Typical serial lines: Analytical derivations}

\section{A.1 Two-machine-one buffer system}

The evolution of the stochastic processes and the corresponding analytical derivations for $N_{2}(t)$ under Case 1, Case 2, and Case 3, in which the first case holds for $p^{1, k}$ parts and the second case for $p^{2, k}$ parts in period $k$, for the two-machine transfer line with finite buffer storage, are given.

Case $1 T_{\lambda}^{i}+T_{\mu_{1}}^{i} \geq T_{\mu_{2}}^{i-1}$ for each part, $i$.

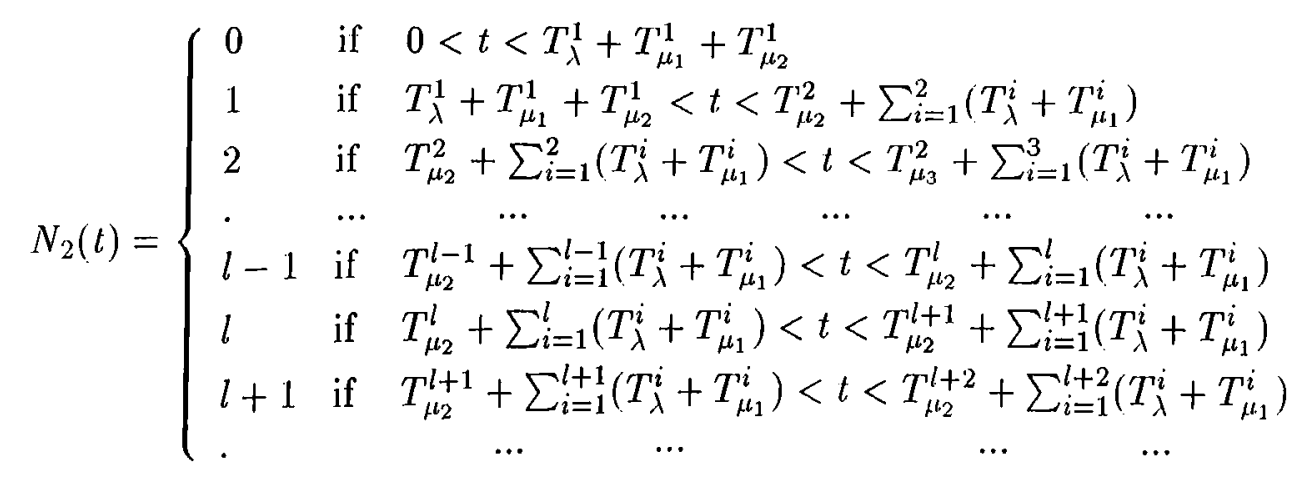




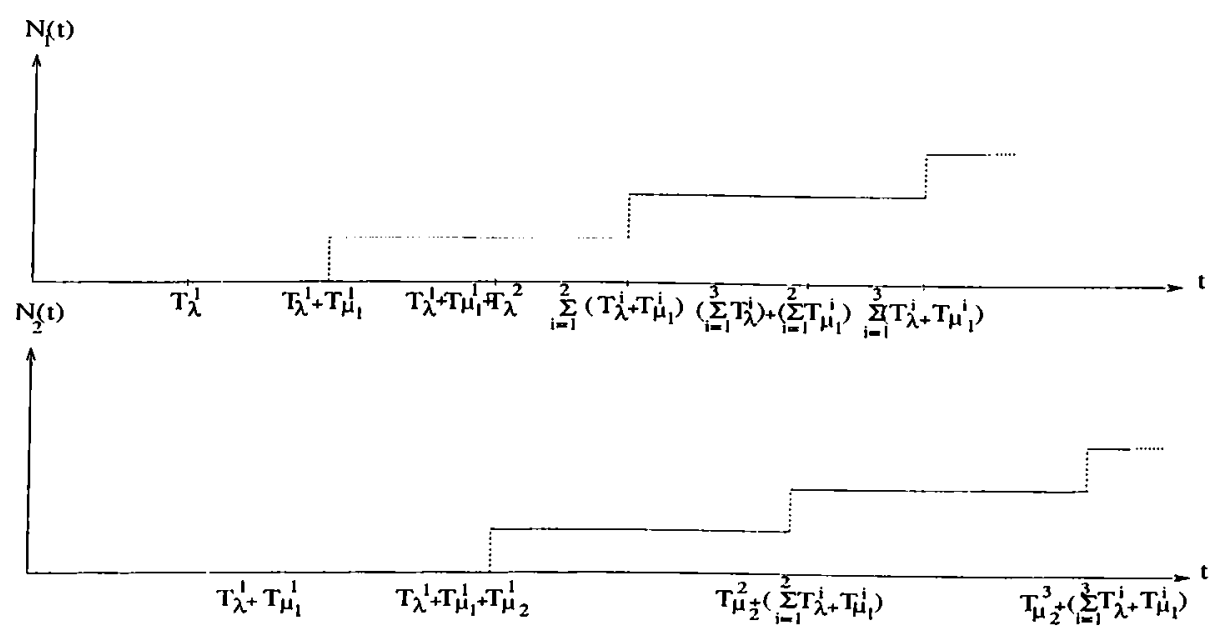

Figure A.1: Evolution of the stochastic process, $N_{1}(t) \& N_{2}(t)$.

Case $2 T_{\lambda}^{i}+T_{\mu_{1}}^{i}<T_{\mu_{2}}^{i-1}$ for each part, $i$.
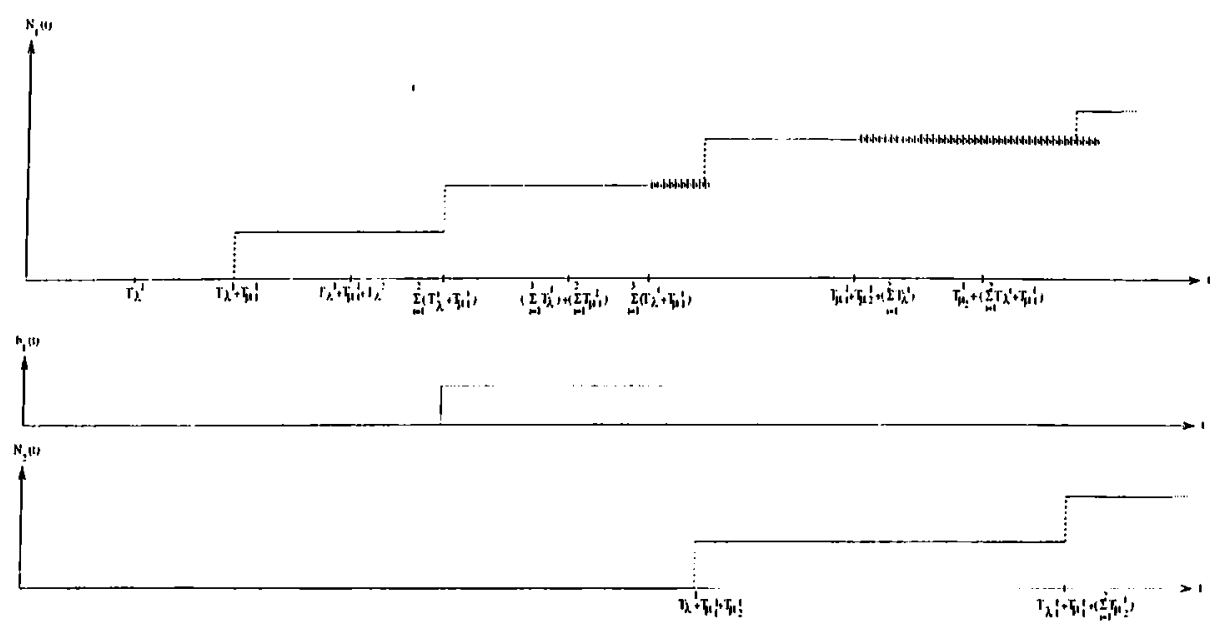

Figure A.2: Evolution of the stochastic process, $N_{1}(t), b_{1}(t), \& N_{2}(t)$.

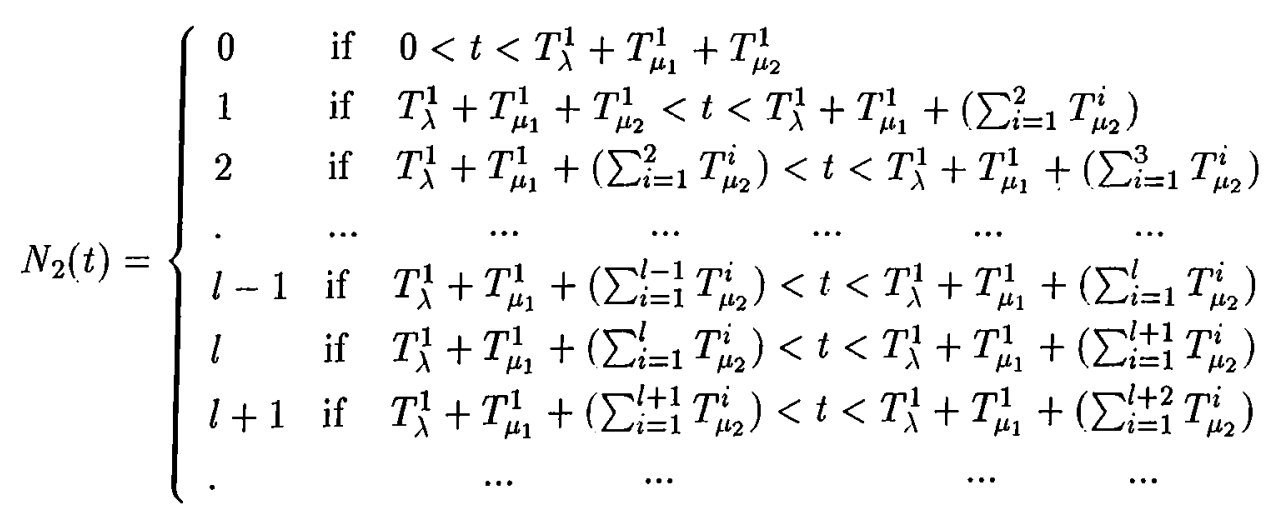




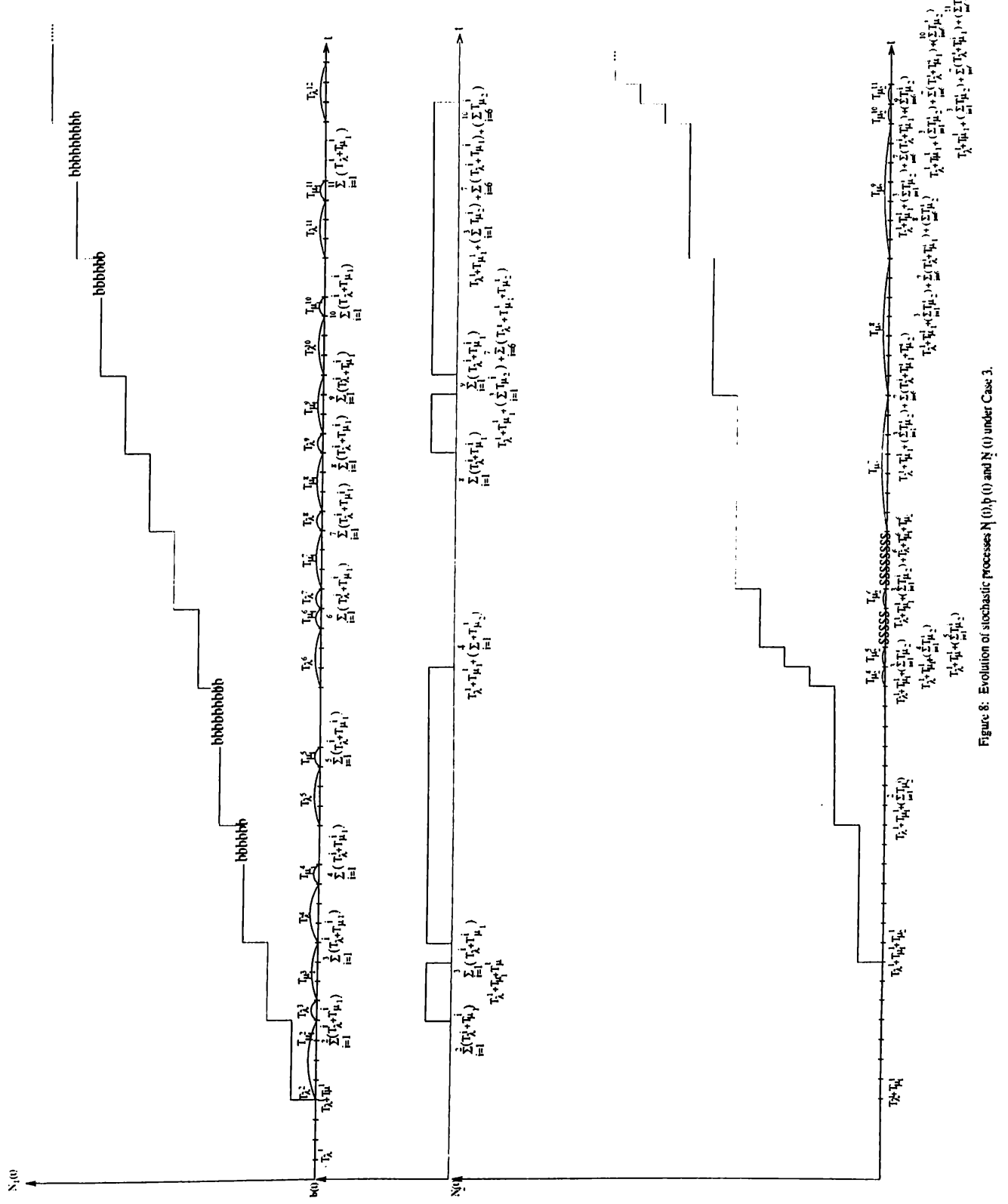

Figure A.3: Evolution of the stochastic processes $\left.N_{1}(t), b_{1}(t), \& N_{2}(t)\right)$. 


\section{APPENDIX A. TYPICAL SERIAL LINES: ANALYTICAL DERIVATIONS111}

Aggregate Case $T_{\lambda}^{i}+T_{\mu_{1}}^{i} \geq T_{\mu_{2}}^{i-1}$ holds for $p^{1, k}$ parts in the $k^{t h}$ period, $T_{\lambda}^{i}+T_{\mu_{1}}^{i}<T_{\mu_{2}}^{i-1}$ holds for $p^{2, k}$ parts in the $k^{t h}$ period.

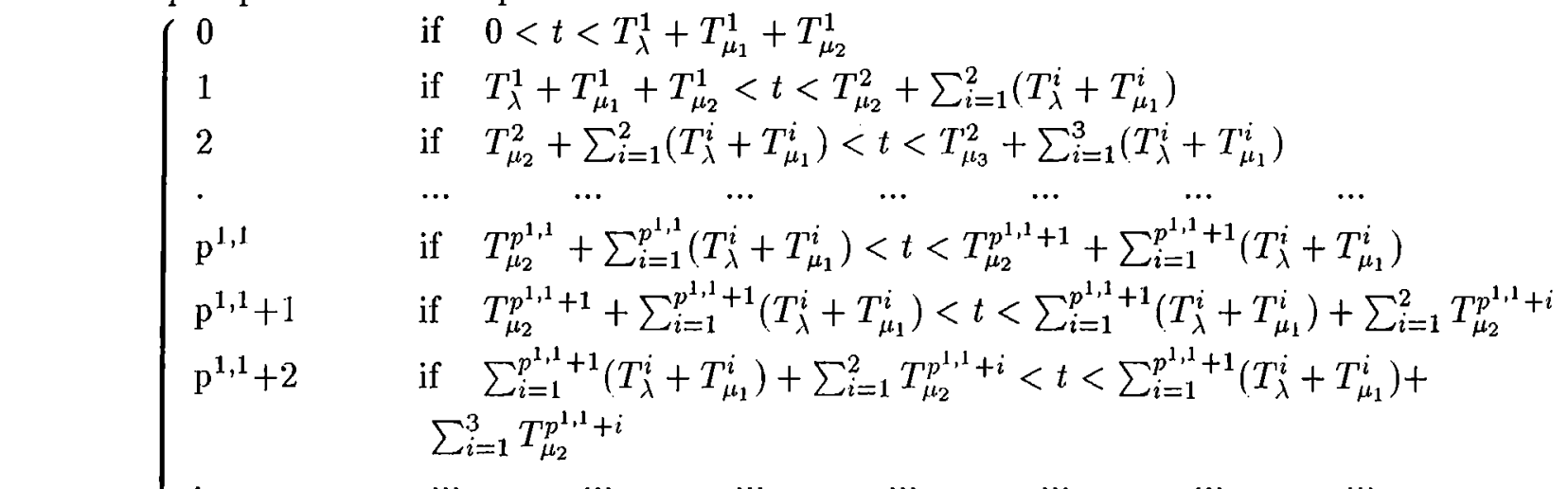

$$
\begin{aligned}
& \mathrm{p}^{1,1}+p^{2,1} \quad \text { if } \quad \sum_{i=1}^{p^{1,1}+1}\left(T_{\lambda}^{i}+T_{\mu_{1}}^{i}\right)+\sum_{i=1}^{p^{2,1}} T_{\mu_{2}}^{p^{1,1}+i}<t<\sum_{i=1}^{p^{1,1}+1}\left(T_{\lambda}^{i}+T_{\mu_{1}}^{i}\right)+ \\
& \sum_{i=1}^{p^{2,1}+1} T_{\mu_{2}}^{p^{1,1}+i} \\
& \mathrm{p}^{1,1}+p^{2,1}+1 \quad \text { if } \quad \sum_{i=1}^{p^{1,1}+1}\left(T_{\lambda}^{i}+T_{\mu_{1}}^{i}\right)+\sum_{i=1}^{p^{2,1}+1} T_{\mu_{2}}^{p^{1,1}+i}<t<\sum_{i=1}^{p^{1,1}+1}\left(T_{\lambda}^{i}+T_{\mu_{1}}^{i}\right)+ \\
& \sum_{i=1}^{p^{2,1}+2} T_{\mu_{2}}^{p^{1,1}+i} \\
& \dot{\mathrm{p}}^{1,1}+p^{2,1}+b_{p^{1,1}+p^{2,1}}^{1}+1
\end{aligned}
$$

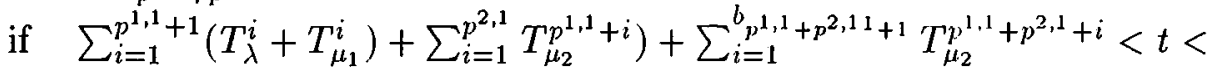

$$
\begin{aligned}
& \sum_{i=1}^{p^{1,1}+1}\left(T_{\lambda}^{i}+T_{\mu_{1}}^{i}\right)+\sum_{i=1}^{b_{b}^{1}+b^{1}+2} T_{\mu_{2}}^{p^{1,1}+i}+\sum_{i=b_{b}^{1}+b^{1}+2}^{p^{1,1}+p^{2,1}+b^{1,1}+1} T_{\mu_{2}}^{i} \\
& N_{2}(t)=\left\{\mathrm{p}^{1,1}+p^{2,1}+b_{p^{1,1}+p^{2,1}}^{1}+2\right. \\
& \text { if } \quad \sum_{i=1}^{p^{1,1}+1}\left(T_{\lambda}^{i}+T_{\mu_{1}}^{i}\right)+\sum_{i=1}^{b_{b}^{1}+b^{1}+2} T_{\mu_{2}}^{p^{1,1}+i}+\sum_{i=b_{b}^{1}+b^{1}+2}^{p^{1,1}+p^{2,1}+b_{p^{1,1}}^{1}+1} T_{\mu_{2}}^{i}<t< \\
& \sum_{i=1}^{p^{1,1}+1}\left(T_{\lambda}^{i}+T_{\mu_{1}}^{i}\right)+\sum_{i=1}^{b_{b}^{1}+b^{1}+2} T_{\mu_{2}}^{p^{1,1}+i}+\sum_{i=b_{b}^{1}+b^{1}+2}^{p^{1,1}+p^{2,1}+b^{1}{ }^{1,1}+2} T_{\mu_{2}}^{i} \\
& \sum_{i=1}^{2}\left(p^{1, i}\right)+p^{2,1} \\
& \text { if } \sum_{i=1}^{p^{1,1}+1}\left(T_{\lambda}^{i}+T_{\mu_{1}}^{i}\right)+\sum_{i=1}^{b_{b}^{1}+b^{1}+2} T_{\mu_{2}}^{p^{1,1}+i}+\sum_{i=1}^{p^{1,2}}\left(T_{\lambda}^{b_{b}^{1}+b^{1}+2+i}+T_{\mu_{1}^{b}}^{b_{b}^{1}+b^{1}+2+i}\right) \\
& +T_{\mu_{2}}^{b_{b}^{1}+b^{1}+2+p^{1,2}}<t<\sum_{i=1}^{p^{1,1}+1}\left(T_{\lambda}^{i}+T_{\mu_{1}}^{i}\right)+\sum_{i=1}^{b_{b}^{1}+b^{1}+2} T_{\mu_{2}}^{p^{1,1}+i}+ \\
& \sum_{i=1}^{p^{1,2}}\left(T_{\lambda}^{b_{b}^{1}+b^{1}+2+i}+T_{\mu_{1}^{b}}^{b_{b}^{1}+b^{1}+2+i}\right)+T_{\mu_{2}}^{b_{b}^{1}+b^{1}+2+p^{1,2}+1} \\
& \sum_{i=1}^{2}\left(p^{1, i}\right)+p^{2,1}+1 \\
& \text { if } \sum_{i=1}^{p^{1,1}+1}\left(T_{\lambda}^{i}+T_{\mu_{1}}^{i}\right)+\sum_{i=1}^{b_{b}^{1}+b^{1}+2} T_{\mu_{2}}^{p^{1,1}+i}+\sum_{i=1}^{p^{1,2}}\left(T_{\lambda}^{b_{b}^{1}+b^{1}+2+i}+T_{\mu_{1}}^{b_{b}^{1}+b^{1}+2+i}\right) \\
& +T_{\mu_{2}}^{b_{b}^{1}+b^{1}+2+p^{1,2}+1}<t<\sum_{i=1}^{p^{1,1}+1}\left(T_{\lambda}^{i}+T_{\mu_{1}}^{i}\right)+\sum_{i=1}^{b_{b}^{1}+b^{1}+2} T_{\mu_{2}}^{p^{1,1}+i}+ \\
& \sum_{i=1}^{p^{1,2}}\left(T_{\lambda}^{b_{b}^{1}+b^{1}+2+i}+T_{\mu_{1}}^{b_{b}^{1}+b^{1}+2+i}\right)+\sum_{i=1}^{2} T_{\mu_{2}}^{b_{b}^{1}+b^{1}+2+p^{1,2}+i} \\
& \sum_{i=1}^{2}\left(p^{1, i}+p^{2, i}\right) \\
& \text { if } \quad \sum_{i=1}^{p^{1,1}+1}\left(T_{\lambda}^{i}+T_{\mu_{1}}^{i}\right)+\sum_{i=1}^{b_{b}^{1}+b^{1}+2} T_{\mu_{2}}^{p^{1,1}+i}+\sum_{i=1}^{p^{1,2}}\left(T_{\lambda}^{b_{b}^{1}+b^{1}+2+i}+T_{\mu_{1}^{b}}^{b_{b}^{1}+b^{1}+2+i}\right) \\
& +\sum_{i=1}^{p^{2,2}} T_{\mu_{2}}^{b_{b}^{1}+b^{1}+2+p^{1,2}+i}<t<\sum_{i=1}^{p^{1,1}+1}\left(T_{\lambda}^{i}+T_{\mu_{1}}^{i}\right)+\sum_{i=1}^{b_{b}^{1}+b^{1}+2} T_{\mu_{2}}^{p^{1,1}+i}+ \\
& \sum_{i=1}^{p^{1,2}}\left(T_{\lambda}^{b_{b}^{1}+b^{1}+2+i}+T_{\mu_{1}}^{b_{b}^{1}+b^{1}+2+i}\right)+\sum_{i=1}^{p^{2,2}+1} T_{\mu_{2}}^{b_{b}^{1}+b^{1}+2+p^{1,2}+i}\left(L H S^{2,2}\right)
\end{aligned}
$$




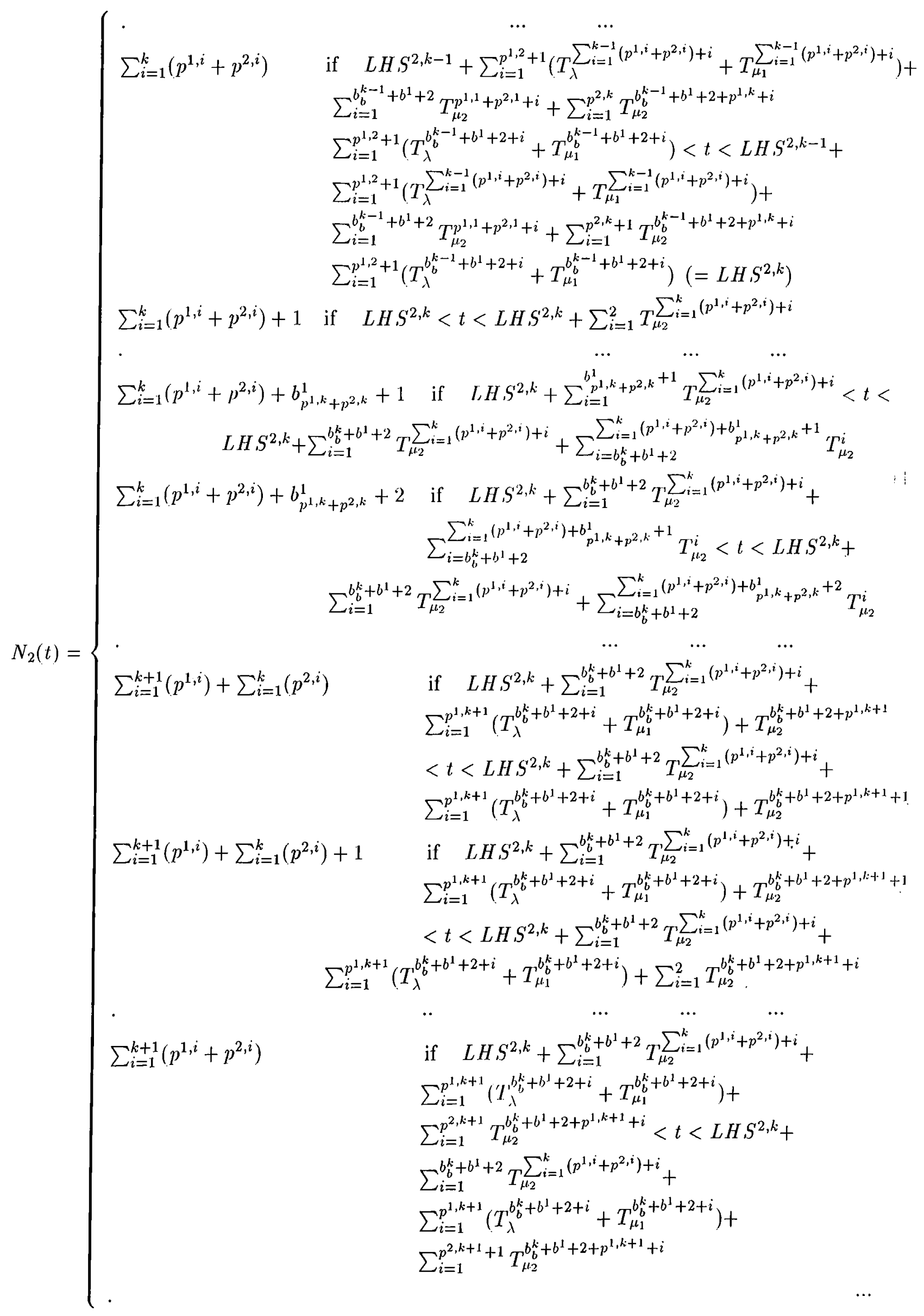


where

$$
\begin{aligned}
& b_{1}^{1}=\left(\max _{x}\left(\sum_{i=1}^{x}\left(T_{\lambda}^{i}+T_{\mu_{1}}^{i}\right) \leq T_{\lambda}^{1}+T_{\mu_{1}}^{1}+T_{\mu_{2}}^{1}\right)-1,\right. \\
& b_{2}^{1}=\left(b_{1}^{1}-1\right)_{\geq 0}+\left(\left(\max _{x}\left(\sum_{i=1}^{x}\left(T_{\lambda}^{i}+T_{\mu_{1}}^{i}\right) \leq T_{\lambda}^{1}+T_{\mu_{1}}^{1}+\sum_{i=1}^{2} T_{\mu_{2}}^{i}\right)-2\right),\right. \\
& b_{k}^{1}=\left(b_{k-1}^{1}-1\right)_{\geq 0}+\left(\left(\max _{x}\left(\sum_{i=1}^{x}\left(T_{\lambda}^{i}+T_{\mu_{1}}^{i}\right) \leq T_{\lambda}^{1}+T_{\mu_{1}}^{1}+\sum_{i=1}^{k} T_{\mu_{2}}^{i}\right)-k\right),\right.
\end{aligned}
$$

if $b_{s}^{1}=\left(b_{s-1}^{1}-1\right) \geq 0+\left(\left(\max _{x}\left(\sum_{i=1}^{x}\left(T_{\lambda}^{i}+T_{\mu_{1}}^{i}\right) \leq T_{\lambda}^{1}+T_{\mu_{1}}^{1}+\sum_{i=1}^{s} T_{\mu_{2}}^{i}\right)-s\right)=b^{1}+1\right.$ then

if $\sum_{i=1}^{s+b^{1}+2}\left(T_{\lambda}^{i}+T_{\mu_{1}}^{i}\right)<T_{\mu_{2}}^{s}$ and $T_{\lambda}^{s+b^{1}+2}+T_{\mu_{1}}^{s+b^{1}+2}<T_{\mu_{2}}^{s+1}$ then

$$
b_{s+1}^{1}=b^{1}+1\{\text { blocked again }\} \text { and } b_{b}^{\text {relevantperiod }}=s
$$

else

$$
b_{s+1}^{1}=b^{1}+1-\left\lfloor\left(T_{\lambda}^{s+b^{1}+2}+T_{\mu_{1}}^{s+b^{1}+2}\right) / T_{\mu_{2}}^{s+1}\right\rfloor
$$

else if $b_{s}^{1}=\left(b_{s-1}^{1}-1\right) \geq 0+\left(\left(\max _{x}\left(\sum_{i=1}^{x}\left(T_{\lambda}^{i}+T_{\mu_{1}}^{i}\right) \leq T_{\lambda}^{1}+T_{\mu_{1}}^{1}+\sum_{i=1}^{s} T_{\mu_{2}}^{i}\right)-\right.\right.$ $s)<b^{1}+1$ then

$$
\begin{aligned}
& b_{s+1}^{1}=\left(b_{s}^{1}-1\right)_{\geq 0}+\left(\left(\operatorname { m a x } _ { x } \left(\sum_{i=1}^{x}\left(T_{\lambda}^{i}+T_{\mu_{1}}^{i}\right) \leq T_{\lambda}^{1}+T_{\mu_{1}}^{1}+\right.\right.\right. \\
& \left.\left.\sum_{i=1]^{s+1}} T_{\mu_{2}}^{i}\right)-(s+1)\right)
\end{aligned}
$$

\section{A.2 Three-machine-two-buffer system}

Case $1 T_{\lambda}^{i}+T_{\mu_{1}}^{i} \geq T_{\mu_{2}}^{i-1}$ and $T_{\lambda}^{i}+T_{\mu_{1}}^{i}+T_{\mu_{2}}^{i} \geq T_{\mu_{2}}^{i-1}+T_{\mu_{3}}^{i-1}$ holds for every part $i$.

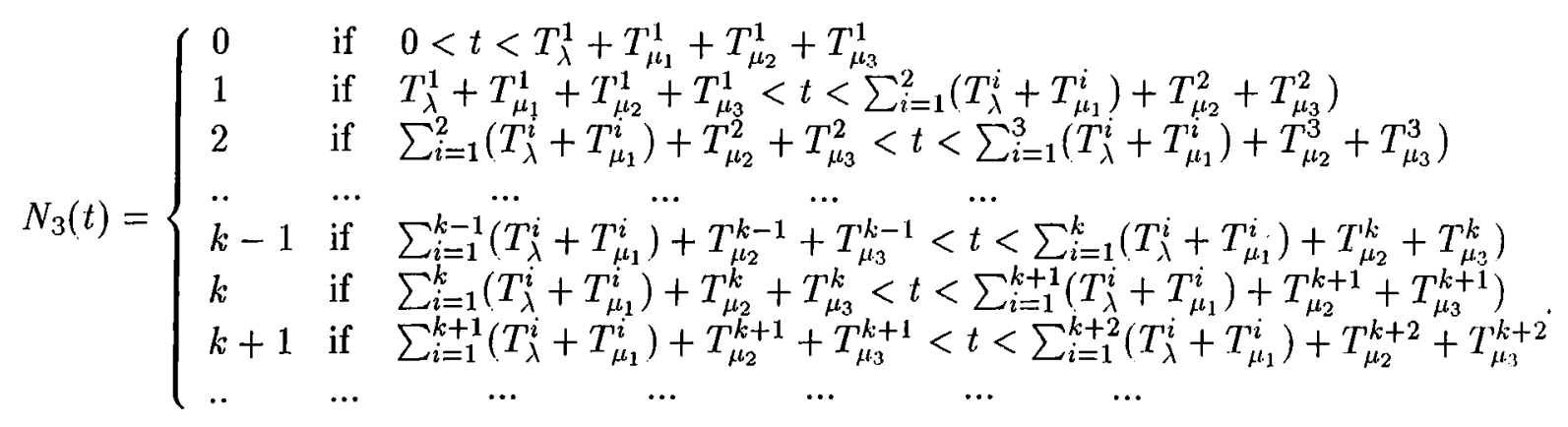




\section{APPENDIX A. TYPICAL SERIAL LINES: ANALYTICAL DERIVATIONS114}

Case $2 T_{\lambda}^{i}+T_{\mu_{1}}^{i} \geq T_{\mu_{2}}^{i-1}$ and $T_{\lambda}^{i}+T_{\mu_{1}}^{i}+T_{\mu_{2}}^{i}<T_{\mu_{2}}^{i-1}+T_{\mu_{3}}^{i-1}$ holds for every part $i$.

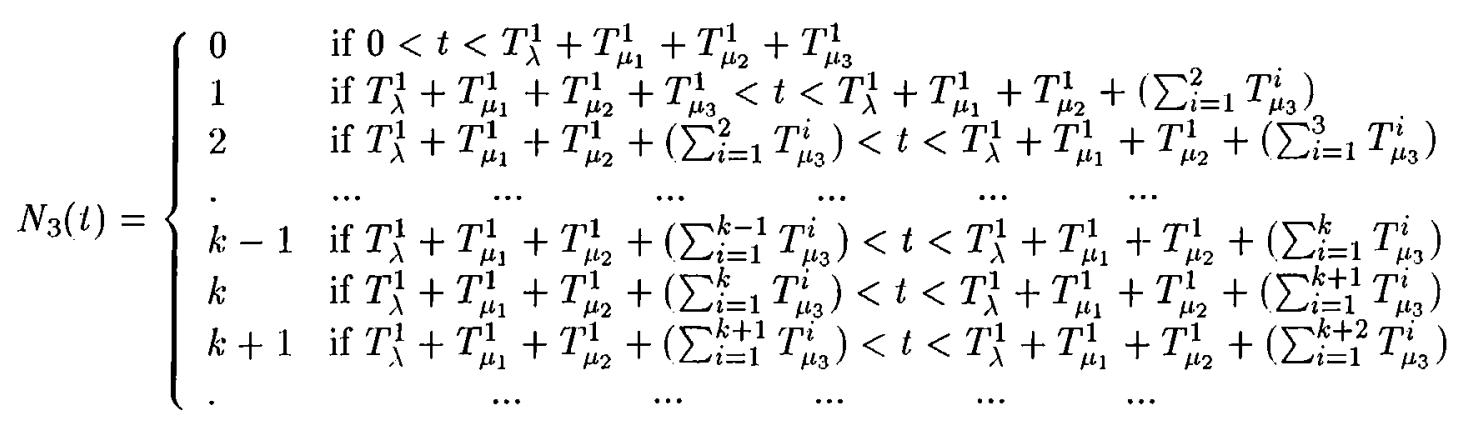

Case $3 T_{\lambda}^{i}+T_{f^{l_{1}}}^{i}<T_{\mu_{2}}^{i-1}$ and $T_{\mu_{2}}^{i-2} \geq T_{\lambda}^{i-1}$ holds for every part $i$.

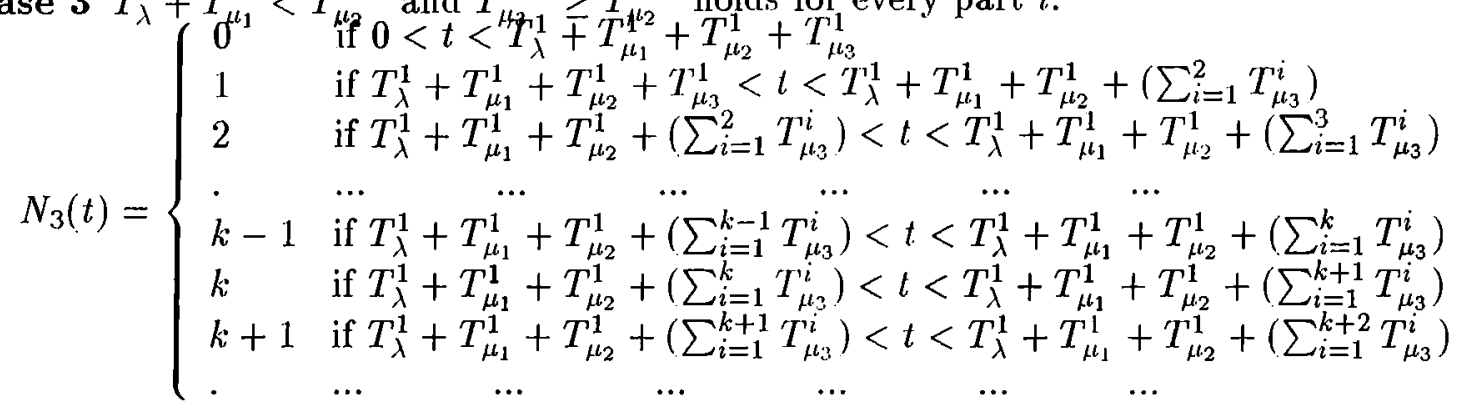

Case $4 T_{\lambda}^{i}+T_{\mu_{1}}^{i}<T_{\mu_{2}}^{i-1}$ and $T_{\mu_{3}}^{i-2}<T_{\mu_{2}}^{i-1}$ holds for every part $i$.

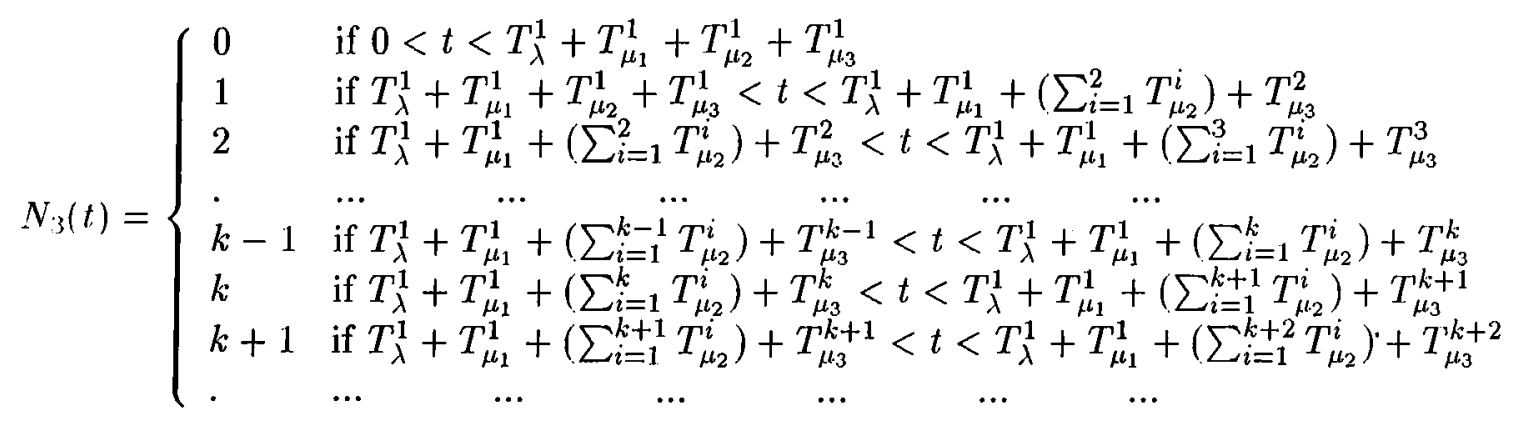

Aggregate Case $T_{\lambda}^{i}+T_{\mu_{1}}^{i} \geq T_{\mu_{2}}^{i-1}$ and $T_{\lambda}^{i}+T_{\mu_{1}}^{i}+T_{\mu_{2}}^{i} \geq T_{\mu_{2}}^{i-1}+T_{\mu_{3}}^{i-1}$ holds for $p^{1, k}$ parts in the $k^{t h}$ period, $T_{\lambda}^{i}+T_{\mu_{1}}^{i} \geq T_{\mu_{2}}^{i-1}$ and $T_{\lambda}^{i}+T_{\mu_{1}}^{i}+T_{\mu_{2}}^{i}<T_{\mu_{2}}^{i-1}+T_{\mu_{3}}^{i-1}$ holds for $p^{2, k}$ parts in the $k^{t h}$ period, $T_{\lambda}^{i}+T_{\mu_{1}}^{i}<T_{\mu_{2}}^{i-1}$ and $T_{\mu_{3}}^{i-2} \geq T_{\mu_{2}}^{i-1}$ holds for $p^{3, k}$ parts in the $k^{t h}$ period, and $T_{\lambda}^{i}+T_{\mu_{1}}^{i}<T_{\mu_{2}}^{i-1}$ and $T_{\mu_{3}}^{i-2}<T_{\mu_{2}}^{i-1}$ holds for $p^{4, k}$ parts in the $k^{\text {th }}$ period. 


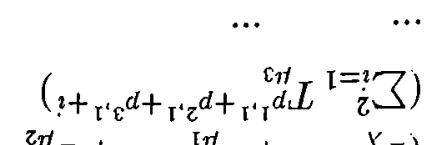

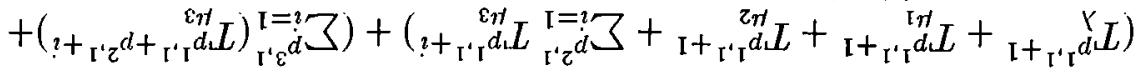

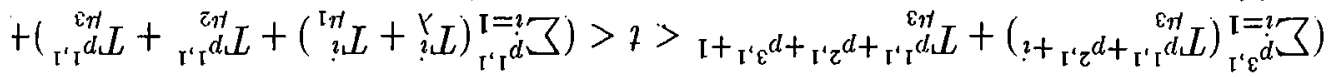

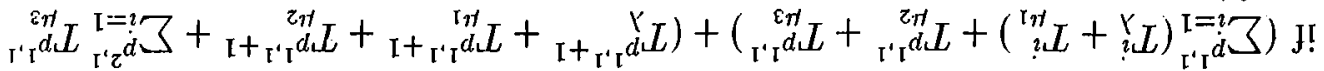

$$
\begin{aligned}
& \mathrm{I}+{ }_{{ }_{\mathrm{I}}{ }^{\prime} \varepsilon} d+{ }_{\mathrm{I}^{\prime} \mathrm{z}} d+{ }_{\mathrm{\gamma}^{\prime} \mathrm{I}} \mathrm{d}
\end{aligned}
$$

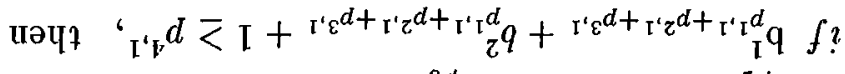

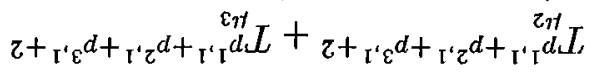

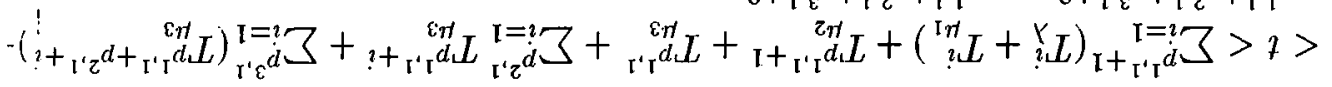

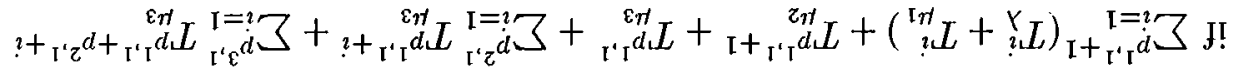

$$
\begin{aligned}
& \mathrm{I}^{\prime} \varepsilon d+{ }_{\mathrm{I}^{\prime}} z^{d+{ }^{\prime} \mathrm{I}} \mathrm{d} \\
& \text {... } \\
& \text {... } \quad \text {... }
\end{aligned}
$$

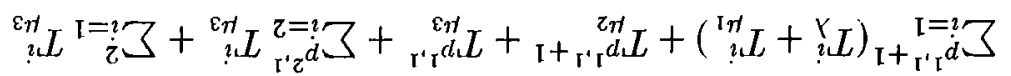

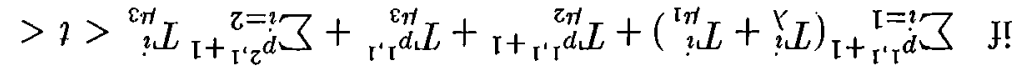

$$
\begin{aligned}
& \mathrm{I}+{ }_{\mathbf{I}^{\prime} z} d+\mathrm{I}^{\prime} \mathrm{I}^{\mathrm{d}}
\end{aligned}
$$

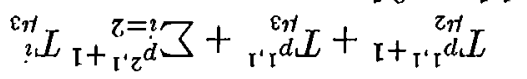

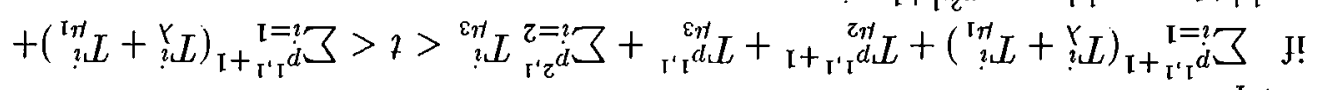

$$
\begin{aligned}
& \mathrm{I}^{\prime} z^{d+{ }_{\mathrm{I}}{ }^{\mathrm{I}} \mathrm{d}} \\
& \text {... }
\end{aligned}
$$

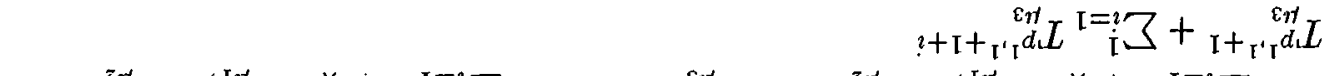

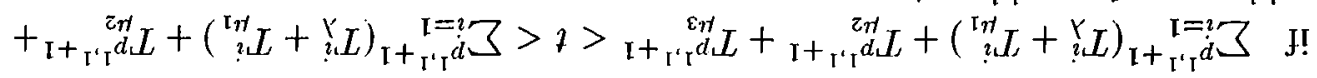

$$
\begin{aligned}
& I+I^{t} I^{d}
\end{aligned}
$$

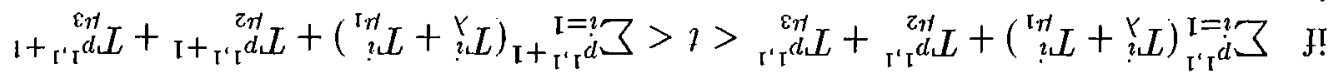

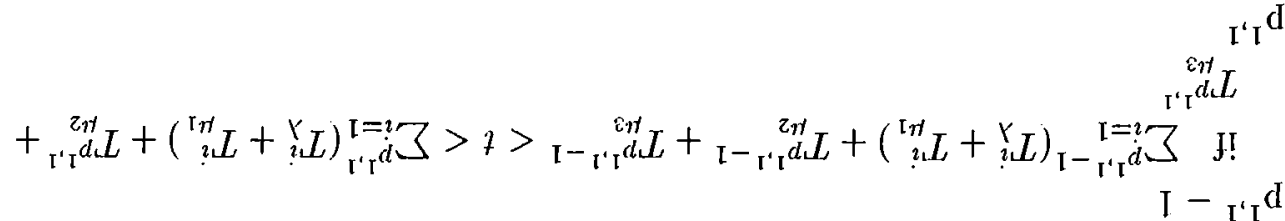

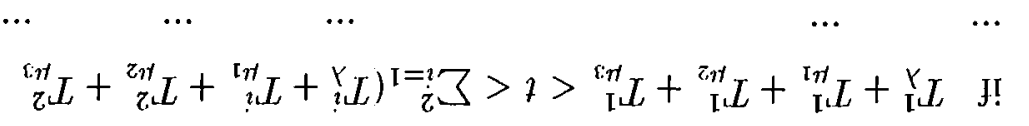

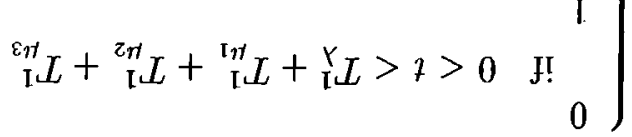




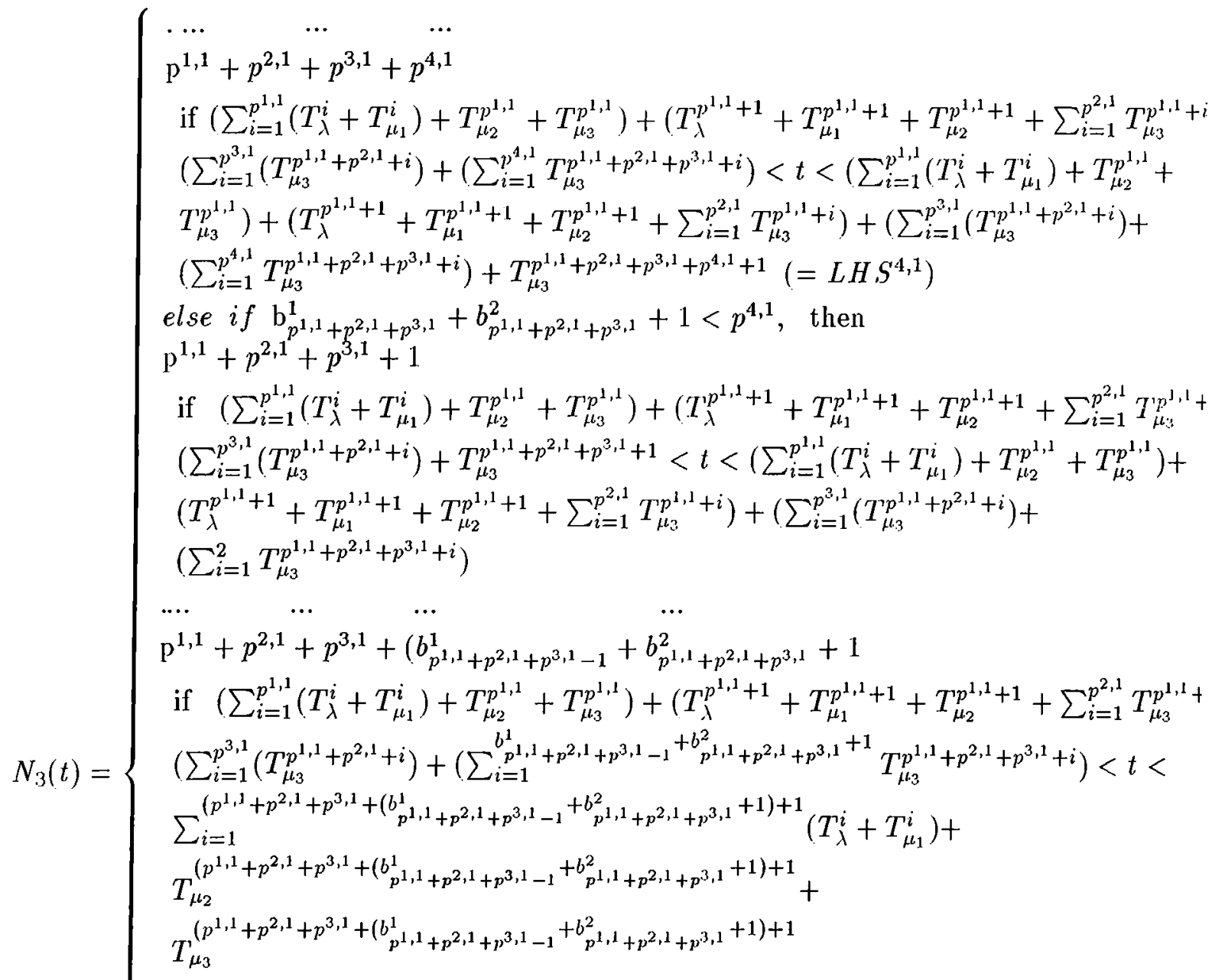

$$
\begin{aligned}
& \mathrm{p}^{1,1}+p^{2,1}+p^{3,1}+p^{4,1} \\
& \text { if } \sum_{i=1}^{\left(p^{1,1}+p^{2,1}+p^{3,1}+\left(b_{p^{1,1}+p^{2,1}+p^{3,1}-1}+b_{p^{1,1}+p^{2,1}+p^{3,1}}^{2}+1\right)+1\right.}\left(T_{\lambda}^{i}+T_{\mu_{\perp}}^{i}\right)+ \\
& \sum_{i=\left(p^{1,1}+p^{2,1}+p^{3,1}+b_{p^{1,1}+p^{2,1}+p^{3,1}-1}^{p^{1,1}+p^{2,1}+b^{1,1}+p^{2,1}+p^{3,1}}+1\right)+1}^{p^{2}+1} T_{\mu_{2}}^{p^{1,1}+p^{2,1}+p^{3,1}+i}+ \\
& T_{\mu_{3}}^{\left.p^{1,1}+p^{2,1}+p^{3,1}+p^{4,1}<t<\sum_{i=1}^{\left(p^{1,1}+p^{2,1}+p^{3,1}+\left(b_{p^{1,1}}^{1}+p^{2,1}+p^{3,1}-1\right.\right.}+b_{p^{1,1}+p^{2,1}+p^{3,1}}^{2}+1\right)+1} \\
& \left(T_{\lambda}^{i}+T_{\mu_{1}}^{i}\right)+T_{\mu_{3}}^{p^{1,1}+p^{2,1}+p^{3,1}+p^{4,1}}+T_{\mu_{3}}^{p^{1,1}+p^{2,1}+p^{3,1}+p^{4,1}+1}
\end{aligned}
$$

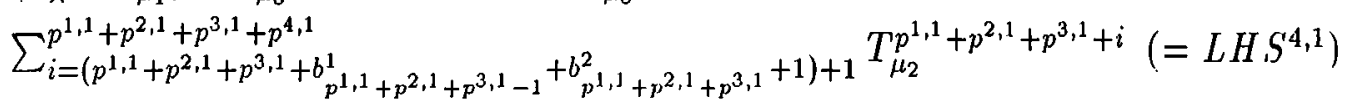

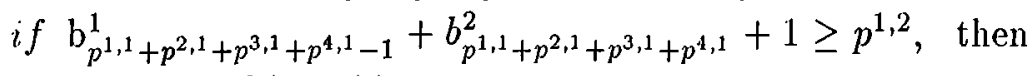

$$
\begin{aligned}
& \mathrm{p}^{1,1}+p^{2,1}+p^{3,1}+p^{4,1}+1 \\
& \text { if } L H S^{4,1}+T_{\mu_{3}}^{1}<t<L H S^{4,1}+\sum_{i=1}^{2} T_{\mu_{3}}^{i}
\end{aligned}
$$




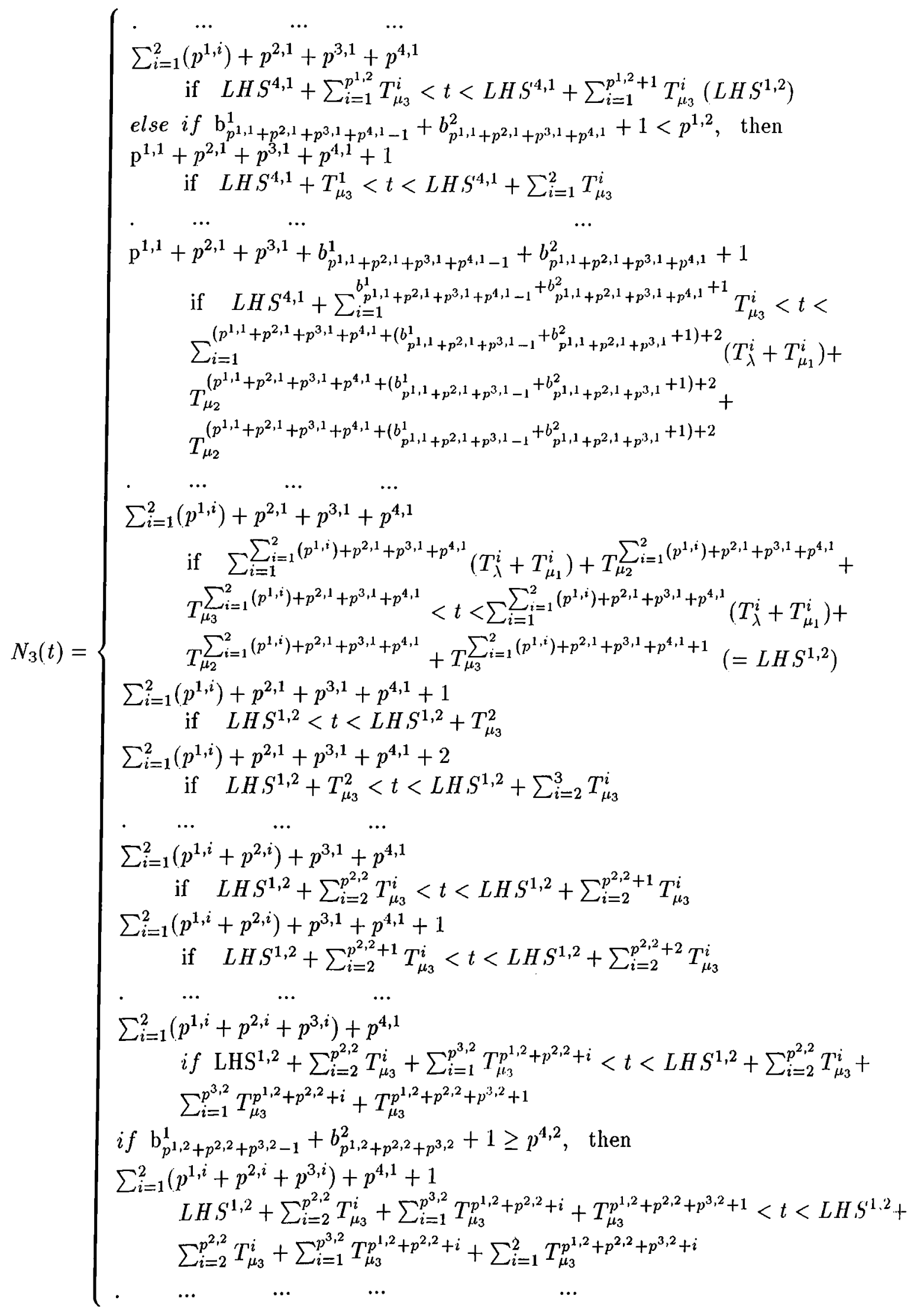




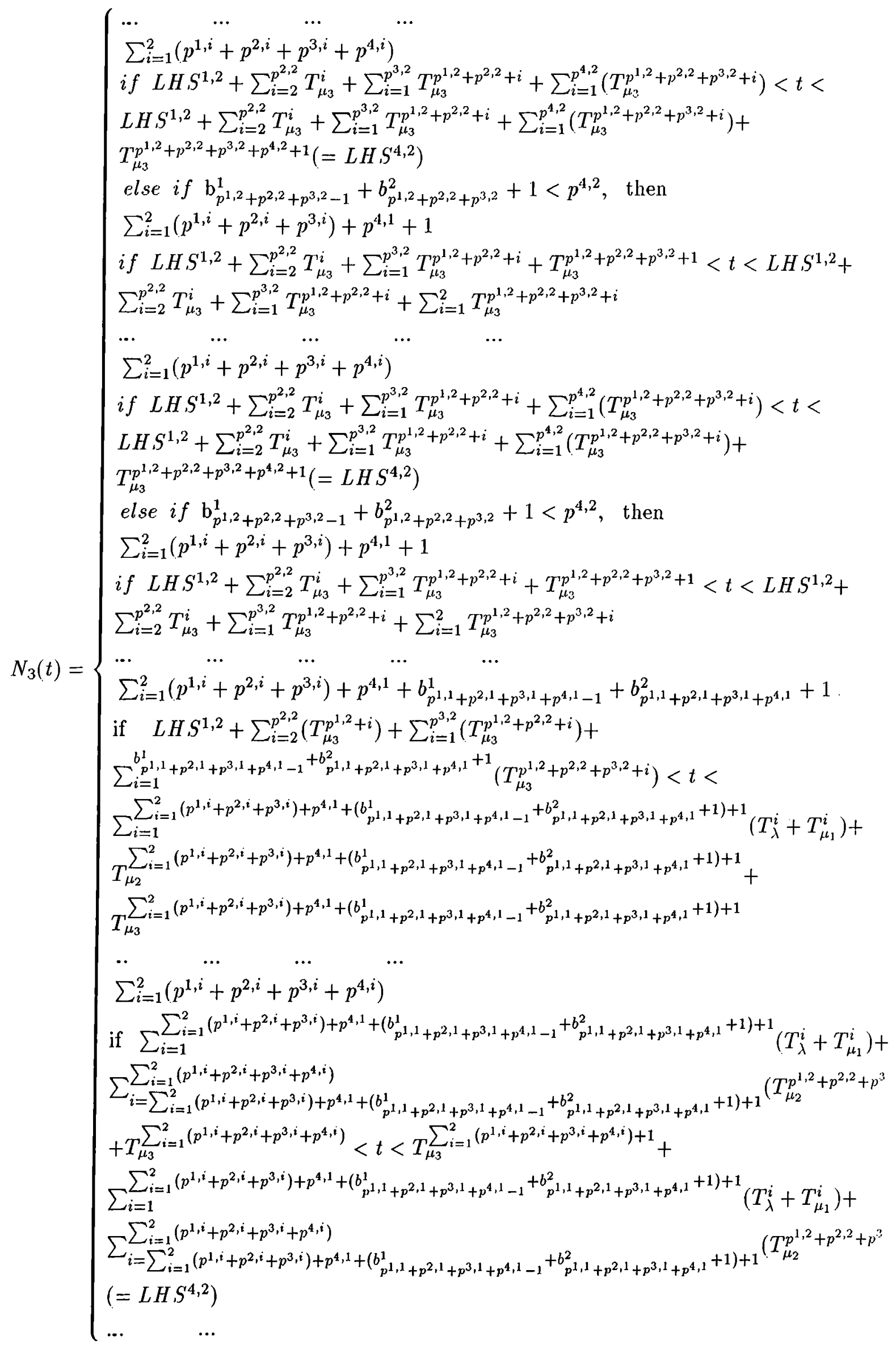




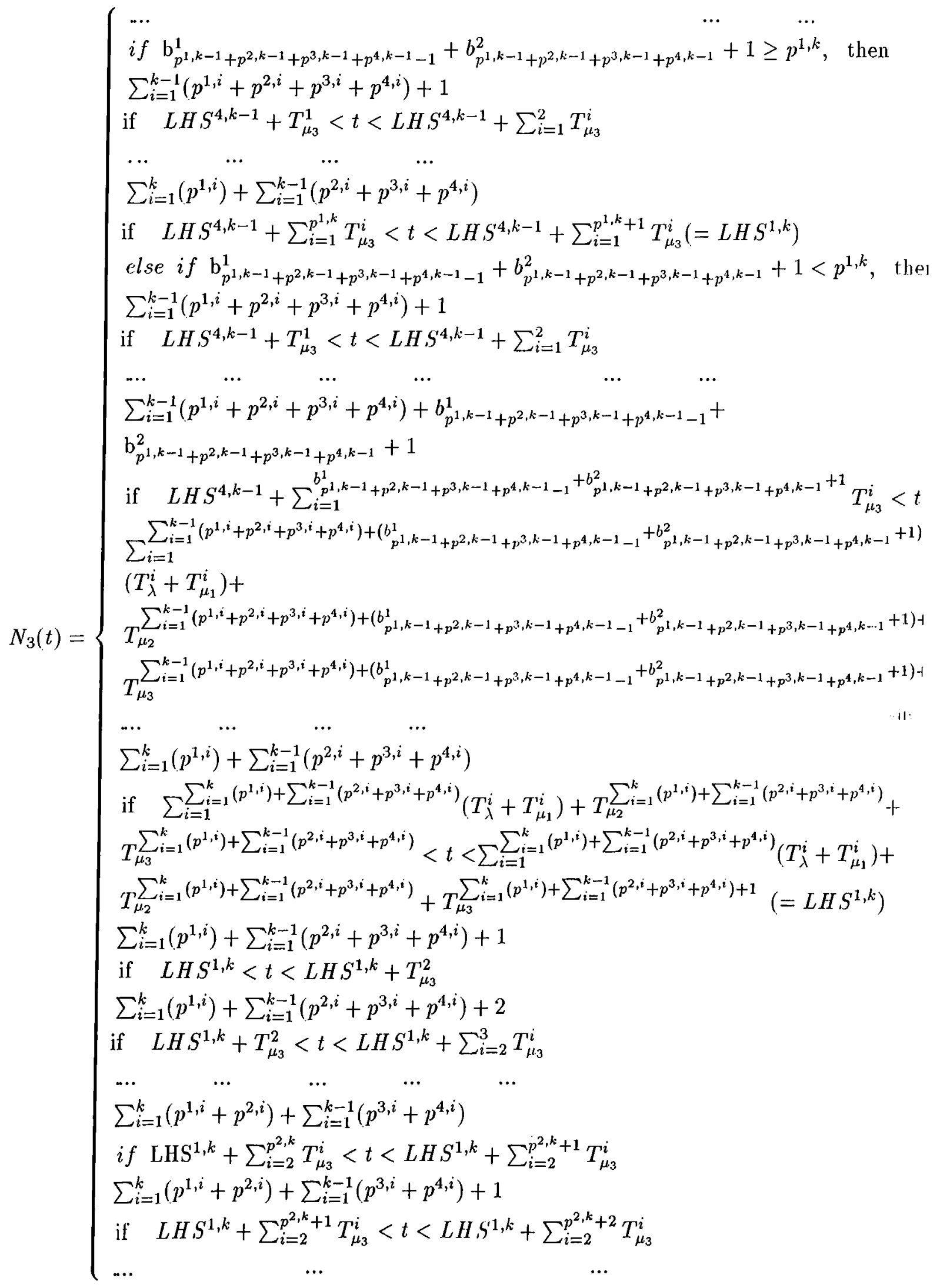




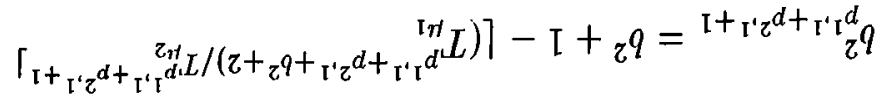

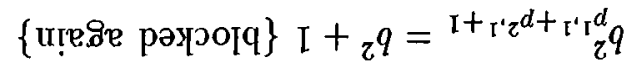

$$
\begin{aligned}
& \text { บวบ7 }
\end{aligned}
$$

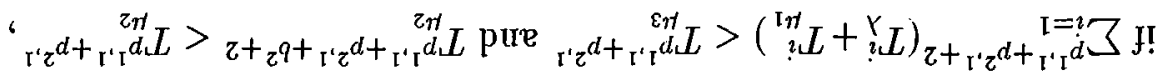

$$
\begin{aligned}
& \text { иәч7 'T }+{ }_{z} q=\mathrm{I}^{\prime} z^{d+{ }_{\mathrm{I}}{ }^{2}}{ }_{z} q \mathrm{~J} \text { ! }
\end{aligned}
$$

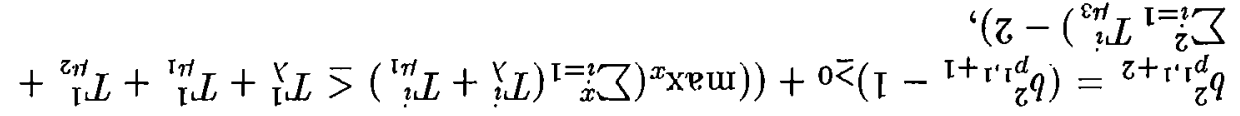

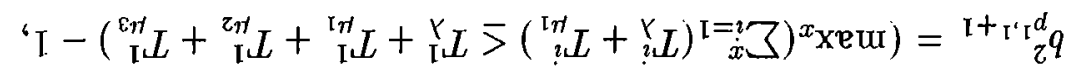

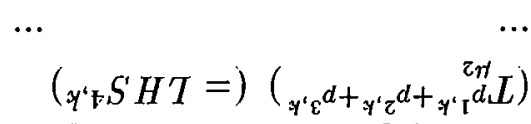

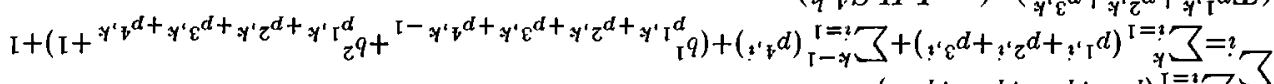

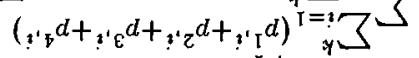

$$
\begin{aligned}
& +\left({ }_{?}^{\mathfrak{r} n} L+{ }_{i}^{\mathrm{r}} L\right)
\end{aligned}
$$

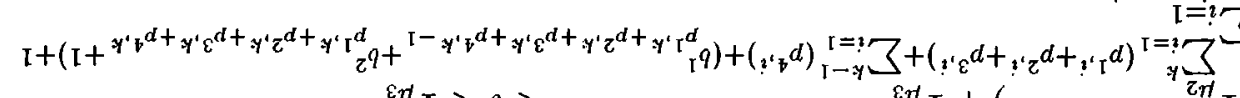

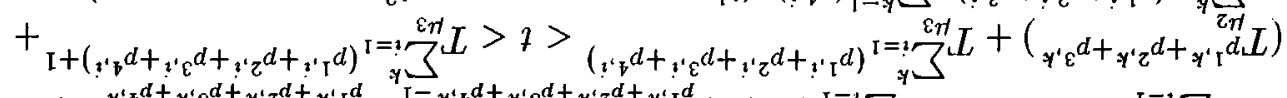

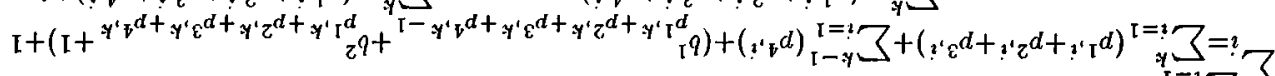

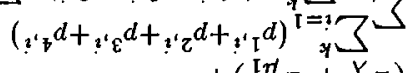

$$
\begin{aligned}
& +\left(\underset{i=?}{i} L+{ }_{i}^{r} L\right)
\end{aligned}
$$

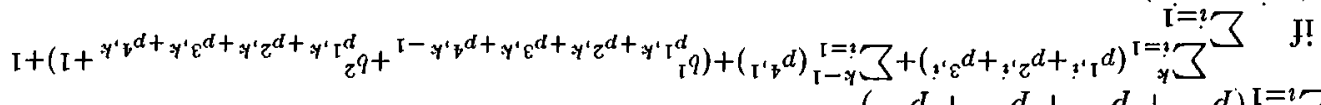

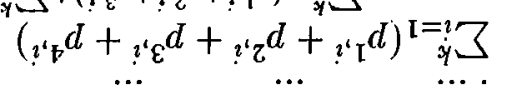

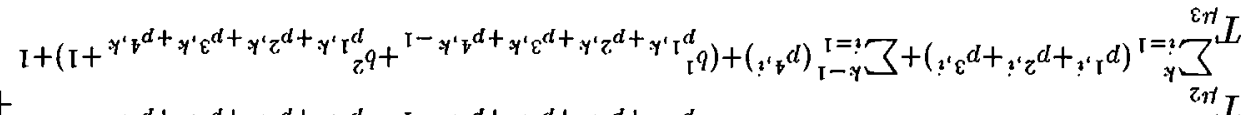

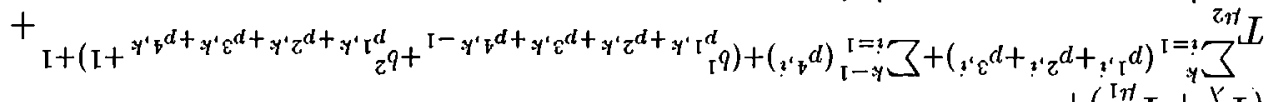

$$
\begin{aligned}
& +\left({ }_{?}^{[n} L+{ }_{i} L\right)
\end{aligned}
$$

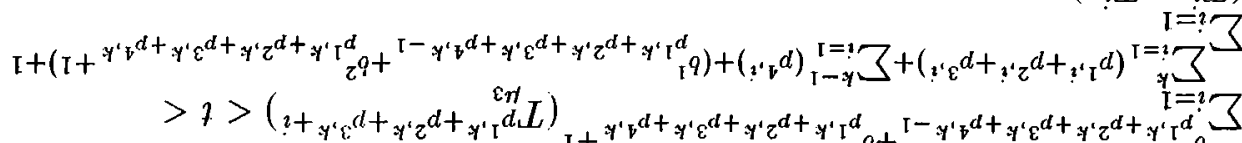

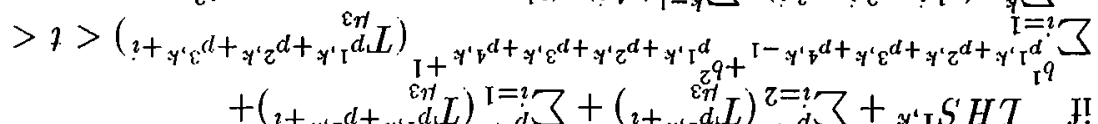

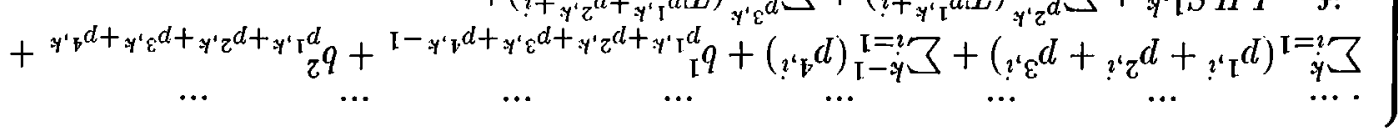




\section{APPENDIX A. TYPICAL SERIAL LINES: ANALYTICAL DERIVATIONS121}

else if $b_{p^{1,1}+p^{2,1}}^{2}<b^{2}+1$, then

$$
\begin{aligned}
& b_{p^{1,1}+p^{2,1}+1}^{2}=\left(b_{p^{1,1}+p^{2,1}+1}^{2}-1\right) \geq 0 \\
& T_{\mu_{1}}^{1}+T_{\mu_{2}}^{1}+\sum_{\left.\left.i=1]^{p^{1,1}+p^{2,1}+1} T_{\mu_{3}}^{i}\right)-\left(p^{1,1}+p^{2,1}+1\right)\right)}
\end{aligned}
$$

if $b_{p^{1,1}+p^{2,1}+p^{3,1}}^{2}=b^{2}+1$, then

$$
\begin{aligned}
& \text { if } \sum_{i=1}^{p^{1,1}+p^{2,1}+p^{3,1}+2}\left(T_{\lambda}^{i}+T_{\mu_{1}}^{i}\right)<T_{\mu_{3}}^{p^{1,1}+p^{2,1}} \text { and } T_{\mu_{2}}^{p^{1,1}+p^{2,1}+p^{3,1}+b^{2}+2}< \\
& T_{\mu_{2}}^{p^{1,1}+p^{2,1}+p^{3,1}}
\end{aligned}
$$

then

$$
\left.b_{p^{1,1}+p^{2,1}+p^{3,1}+1}^{2}=b^{2}+1 \text { blocked again }\right\}
$$

else

$$
b_{p^{1,1}+p^{2,1}+p^{2,1}+1}^{2}=b^{2}+1-\left\lfloor\left( T_{\mu_{1}}^{\left.\left.p^{1,1}+p^{2,1}+p^{3,1}+b^{2}+2\right) / T_{\mu_{2}}^{p^{1,1}+p^{2,1}+p^{3,1}+1}\right\rfloor}\right.\right.
$$

else if $b_{p^{1,1}+p^{2,1}+p^{3,1}}^{2}<b^{2}+1$, then

$$
\begin{aligned}
& b_{p^{1,1}+p^{2,1}+p^{3,1}+1}^{2}=\left(b_{p^{1,1}+p^{2,1}+p^{3,1}+1}^{2}-1\right)_{\geq 0}+\left(\left(\max _{x}\left(\sum_{i=1}^{x} T_{\mu_{2}}^{p^{1,1}+p^{2,1}+p^{3,1}+i}\right) \leq\right.\right. \\
& \left.\left.\sum_{i=1}^{p^{1,1}+p^{2,1}+p^{3,1}+1} T_{\mu_{3}}^{i}\right)-\left(p^{1,1}+p^{2,1}+p^{3,1}+1\right)\right) \\
& \quad \ldots \\
& b_{p^{1,1}+p^{2,1}+p^{3,1}+p^{4,1}+1}^{2}=\left(b_{p^{1,1}+p^{2,1}+p^{3,1}+p^{1,1}-1}^{2}\right)_{\geq 0}+\left(\left(\max _{x}\left(\sum_{i=1}^{x} T_{\mu_{2}}^{p^{1,1}+p^{2,1}+p^{3,1}+p^{4,1}+i}\right)\right.\right. \\
& \left.\left.\leq \sum_{i=1}^{p^{1,1}+p^{2,1}+p^{3,1}+p^{4,1}+1} T_{\mu_{3}}^{i}\right)-\left(p^{1,1}+p^{2,1}+p^{3,1}+p^{4,1}+1\right)\right)
\end{aligned}
$$




\section{A.3 Formulations for the descriptions of the system behavior}

\section{A.3.1 Atomic model}

$$
\begin{aligned}
& E\left(N_{1}(t)\right)=\sum_{l=1}^{\infty} l\left[\frac{\lambda^{l} \mu_{1}^{l} \sum_{i=1}^{l}(-1)^{i+1} A_{i}\left(\lambda-\mu_{1}\right)^{l-i}}{(l-1) !\left(\lambda-\mu_{1}\right)^{2 l-1}}\left(\frac{\Gamma(l-i, t)}{\mu_{1}^{l-i}}-(-1)^{l-i} \frac{\Gamma(l-i, t)}{\lambda_{1}^{l-i}}\right)\right. \\
& \left.-\frac{\lambda^{l+1} \mu_{1}^{l+1} \sum_{i=1}^{l+1}(-1)^{i+1} A_{i}\left(\lambda-\mu_{1}\right)^{l-i+1}}{l !\left(\lambda-\mu_{1}\right)^{2 l+1}}\left(\frac{\Gamma(l-i+1, t)}{\mu_{1}^{l+1-i}}-(-1)^{l+1-i} \frac{\Gamma(l+1-i, t)}{\lambda_{1}^{l+1-i}}\right)\right] \\
& E\left(N_{1}(t)\left(N_{1}(t)-1\right)\right)=\sum_{l=2}^{\infty} l(l-1)\left[\frac { \lambda ^ { l } \mu _ { 1 } ^ { l } \sum _ { i = 1 } ^ { l } ( - 1 ) ^ { i + 1 } A _ { i } ( \lambda - \mu _ { 1 } ) ^ { l - i } } { ( l - 1 ) ! ( \lambda - \mu _ { 1 } ) ^ { 2 l - 1 } } \left(\frac{\Gamma(l-i, t)}{\mu_{1}^{l-i}}\right.\right. \\
& \left.-(-1)^{l-i} \frac{\Gamma(l-i, t)}{\lambda_{1}^{l-i}}\right)-\frac{\lambda^{l+1} \mu_{1}^{l+1} \sum_{i=1}^{l+1}(-1)^{i+1} A_{i}\left(\lambda-\mu_{1}\right)^{l-i+1}}{l !\left(\lambda-\mu_{1}\right)^{2 l+1}}\left(\frac{\Gamma(l-i+1, t)}{\mu_{1}^{l+1-i}}\right. \\
& \left.\left.-(-1)^{l+1-i} \frac{\Gamma(l+1-i, t)}{\lambda_{1}^{l+1-i}}\right)\right] \\
& V\left(N_{1}(t)\right)=E\left(N_{1}(t)\left(N_{1}(t)-1\right)\right)+E\left(N_{1}(t)\right)-E\left(N_{1}(t)\right)^{2} \\
& E(t)=E\left(\frac{N_{1}(t)}{t}\right)=\frac{E\left(N_{1}(t)\right)}{t} \\
& V(t)=V\left(\frac{N_{1}(t)}{t}\right)=\frac{V\left(N_{1}(t)\right)}{t^{2}} \\
& E=\lim _{t \rightarrow \infty} E(t) \\
& V=\lim _{t \rightarrow \infty} V(t)
\end{aligned}
$$

where

$A_{i}$ : Coefficients obtained by the convolution of hypoexponentially distributed tandom variables

$\Gamma$ : Incomplete gamma function 


\section{A.3.2 Two-machine-one-buffer model}

\section{Case 1}

$$
\begin{gathered}
E\left(N_{2}(t)\right)=\sum_{l=1}^{\infty} l\left[\frac{\lambda^{l} \mu_{1}^{l} \mu_{2}}{(l-1) !\left(\lambda-\mu_{1}\right)^{2 l-1}} \sum_{i=1}^{l}(-1)^{i+1} A_{i}\left(\lambda-\mu_{1}\right)^{l-i}\left(Z_{2}\left(l-i, \mu_{1}-\mu_{2}\right)-Z_{2}\left(l-i, \lambda-\mu_{2}\right)\right)\right. \\
\left.\frac{\lambda^{l+1} \mu_{1}^{l+1} \mu_{2}}{l !\left(\lambda-\mu_{1}\right)^{2 l+1}} \sum_{i=1}^{l+1}(-1)^{i+1} A_{i}\left(\lambda-\mu_{1}\right)^{l+1-i}\left(Z_{2}\left(l+1-i, \mu_{1}-\mu_{2}\right)-Z_{2}\left(l+1-i, \lambda-\mu_{2}\right)\right)\right] \\
E\left(N_{2}(t)\left(N_{2}(t)-1\right)\right)=\sum_{l=2}^{\infty} l(l-1)\left[\frac { \lambda ^ { l } \mu _ { 1 } ^ { l } \mu _ { 2 } } { ( l - 1 ) ! ( \lambda - \mu _ { 1 } ) ^ { 2 l - 1 } } \sum _ { i = 1 } ^ { l } ( - 1 ) ^ { i + 1 } A _ { i } ( \lambda - \mu _ { 1 } ) ^ { l - i } \left(Z_{2}\left(l-i, \mu_{1}-\mu_{2}\right)\right.\right. \\
\left.-Z_{2}\left(l-i, \lambda-\mu_{2}\right)\right)-\frac{\lambda^{l+1} \mu_{1}^{l+1} \mu_{2}}{l !\left(\lambda-\mu_{1}\right)^{2 l+1}} \sum_{i=1}^{l+1}(-1)^{i+1} A_{i}\left(\lambda-\mu_{1}\right)^{l+1-i}\left(Z_{2}\left(l+1-i, \mu_{1}-\mu_{2}\right)\right. \\
\left.\left.-Z_{2}\left(l+1-i, \lambda-\mu_{2}\right)\right)\right] \\
V\left(N_{2}(t)\right)=E\left(N_{2}(t)\left(N_{2}(t)-1\right)\right)+E\left(N_{2}(t)\right)-E\left(N_{2}(t)\right)^{2} \\
E(t)=E\left(\frac{N_{2}(t)}{t}\right)=\frac{E\left(N_{2}(t)\right)}{t} \\
V(t)=V\left(\frac{N_{2}(t)}{t}\right)=\frac{V\left(N_{2}(t)\right)}{t^{2}} \\
E=\lim _{t \rightarrow \infty} E(t) \\
V=\lim _{t \rightarrow \infty} V(t)
\end{gathered}
$$

where

$$
Z_{2}(x, y)=\frac{1}{y^{x}} \int_{0}^{t} \Gamma(x+1, y s) e^{-\mu_{2} s} \mathrm{~d} s
$$


Case 2

$$
\begin{gathered}
E\left(N_{2}(t)\right)=\sum_{l=1}^{\infty} l\left[\frac { \lambda \mu _ { 1 } \mu _ { 2 } ^ { l } } { ( l - 1 ) ! ( \mu _ { 1 } - \lambda ) } \left(\frac{\Gamma(l+1, t)}{l \lambda^{l+1}}-\left(Z_{1}\left(l-1, \mu_{1}-\lambda\right)\right)\right.\right. \\
-\frac{\lambda \mu_{1} \mu_{2}^{l+1}}{l !\left(\mu_{1}-\lambda\right)}\left(\frac{\Gamma(l+2, t)}{(l+1) \lambda^{l+2}}-\left(Z_{1}\left(l, \mu_{1}-\lambda\right)\right)\right] \\
E\left(N_{2}(t)\left(N_{2}(t)-1\right)\right)=\sum_{l=2}^{\infty} l(l-1)\left[\frac { \lambda \mu _ { 1 } \mu _ { 2 } ^ { l } } { ( l - 1 ) ! ( \mu _ { 1 } - \lambda ) } \left(\frac{\Gamma(l+1, t)}{l \lambda^{l+1}}-\left(Z_{1}\left(l-1, \mu_{1}-\lambda\right)\right)\right.\right. \\
-\frac{\lambda \mu_{1} \mu_{2}^{l+1}}{l !\left(\mu_{1}-\lambda\right)}\left(\frac{\Gamma(l+2, t)}{\left.(l+1) \lambda^{l+2}-\left(Z_{1}\left(l, \mu_{1}-\lambda\right)\right)\right]}\right. \\
\left.V\left(N_{2}(t)\right)=E_{2}(t)\left(N_{2}(t)-1\right)\right)+E\left(N_{2}(t)\right)-E\left(N_{2}(t)\right)^{2} \\
E(t)=E\left(\frac{N_{2}(t)}{t}\right)=\frac{E\left(N_{2}(t)\right)}{t} \\
V(t)=V\left(\frac{N_{2}(t)}{t}\right)=\frac{V\left(N_{2}(t)\right)}{t^{2}} \\
E=\lim _{t \rightarrow \infty} E(t) \\
V(t) \\
V\left(\lim _{t \rightarrow \infty}\right) \\
V
\end{gathered}
$$

where

$$
Z_{1}(x, y)=\frac{1}{y^{x}} \int_{0}^{t} \Gamma(x+1, y s) e^{-\mu_{1} s} \mathrm{~d} s
$$

\section{A.3.3 Three-machine-one-buffer model}

Case 1

$$
\begin{aligned}
& E\left(N_{3}(t)\right)=\sum_{l=1}^{\infty} l\left[\frac { \lambda ^ { l } \mu _ { 1 } ^ { l } \mu _ { 2 } \mu _ { 3 } } { ( l - 1 ) ! ( \lambda - \mu _ { 1 } ) ^ { 2 l - 1 } ( \mu _ { 3 } - \mu _ { 2 } ) } \sum _ { i = 1 } ^ { l } ( - 1 ) ^ { i + 1 } A _ { i } ( \lambda - \mu _ { 1 } ) ^ { l - i } \left(Z_{2}\left(l-i, \mu_{1}-\mu_{2}\right)\right.\right. \\
& \left.-Z_{3}\left(l-i, \mu_{1}-\mu_{3}\right)-(-1)^{l-1+i}\left(Z_{2}\left(l-i, \lambda-\mu_{2}\right)+Z_{3}\left(l-i, \lambda-\mu_{3}\right)\right)\right)-\frac{\lambda^{l+1} \mu_{1}^{l+1} \mu_{2} \mu_{3}}{l !\left(\lambda-\mu_{1}\right)^{2 l+1}\left(\mu_{3}-\mu_{2}\right)}
\end{aligned}
$$




$$
\begin{aligned}
& \sum_{i=1}^{l+1}(-1)^{i+1} A_{i}\left(\lambda-\mu_{1}\right)^{l+1-i}\left(Z_{2}\left(l+1-i, \mu_{1}-\mu_{2}\right)-Z_{3}\left(l+1-i, \mu_{1}-\mu_{3}\right)-(-1)^{l+i}\left(Z_{2}\left(l+1-i, \lambda-\mu_{2}\right)\right.\right. \\
& \left.\left.+Z_{3}\left(l+1-i, \lambda-\mu_{3}\right)\right)\right) \\
& E\left(N_{3}(t)\left(N_{3}(t)-1\right)\right)=\sum_{l=2}^{\infty} l(l-1)\left[\frac{\lambda^{l} \mu_{1}^{l} \mu_{2} \mu_{3}}{(l-1) !\left(\lambda-\mu_{1}\right)^{2 l-1}\left(\mu_{3}-\mu_{2}\right)} \sum_{i=1}^{l}(-1)^{i+1} A_{i}\left(\lambda-\mu_{1}\right)^{l-i}\right. \\
& \left(Z_{2}\left(l-i, \mu_{1}-\mu_{2}\right)-Z_{3}\left(l-i, \mu_{1}-\mu_{3}\right)-(-1)^{l-1+i}\left(Z_{2}\left(l-i, \lambda-\mu_{2}\right)+Z_{3}\left(l-i, \lambda-\mu_{3}\right)\right)\right)- \\
& \frac{\lambda^{l+1} \mu_{1}^{l+1} \mu_{2} \mu_{3}}{l !\left(\lambda-\mu_{1}\right)^{2 l+1}\left(\mu_{3}-\mu_{2}\right)} \sum_{i=1}^{l+1}(-1)^{i+1} A_{i}\left(\lambda-\mu_{1}\right)^{l+1-i}\left(Z_{2}\left(l+1-i, \mu_{1}-\mu_{2}\right)-Z_{3}\left(l+1-i, \mu_{1}-\mu_{3}\right)-:\right. \\
& \left.(-1)^{l+i}\left(Z_{2}\left(l+1-i, \lambda-\mu_{2}\right)+Z_{3}\left(l+1-i, \lambda-\mu_{3}\right)\right)\right) \\
& V\left(N_{3}(t)\right)=E\left(N_{3}(t)\left(N_{3}(t)-1\right)\right)+E\left(N_{3}(t)\right)-E\left(N_{3}(t)\right)^{2} \\
& E(t)=E\left(\frac{N_{3}(t)}{t}\right)=\frac{E\left(N_{3}(t)\right)}{t} \\
& V(t)=V\left(\frac{N_{3}(t)}{t}\right)=\frac{V\left(N_{3}(t)\right)}{t^{2}} \\
& E=\lim _{t \rightarrow \infty} E(t) \\
& V=\lim _{t \rightarrow \infty} V(t)
\end{aligned}
$$

where

$$
\begin{aligned}
& Z_{2}(x, y)=\frac{1}{y^{x}} \int_{0}^{t} \Gamma(x+1, y s) e^{-\mu_{2} s} \mathrm{ds} \\
& Z_{3}(x, y)=\frac{1}{y^{x}} \int_{0}^{t} \Gamma(x+1, y s) e^{-\mu_{3} s} \mathrm{ds}
\end{aligned}
$$




\section{Case 2}

$$
\begin{aligned}
& E\left(N_{3}(t)\right)=\sum_{l=1}^{\infty} l\left[\frac { \lambda \mu _ { 1 } \mu _ { 2 } \mu _ { 3 } ^ { l } } { ( l - 1 ) ! ( \lambda - \mu _ { 1 } ) } \left(\frac{1}{\left(\mu_{1}-\mu_{2}\right)\left(\lambda-\mu_{2}\right)^{l-1}} Z_{2}\left(l-1, \lambda-\mu_{2}\right)-\frac{1}{\left(\mu_{1}-\mu_{2}\right)\left(\lambda-\mu_{1}\right)^{l-1}}\right.\right. \\
& \left.Z_{1}\left(l-1, \lambda-\mu_{1}\right)-\frac{1}{\left(\lambda-\mu_{2}\right)^{l}} Z_{2}\left(l-1, \lambda-\mu_{2}\right)+\frac{1}{l\left(\lambda-\mu_{2}\right)} \frac{t^{l+1}}{l+1}\right)-\frac{\lambda \mu_{1} \mu_{2} \mu_{3}^{l+1}}{l !\left(\lambda-\mu_{1}\right)}\left(\frac{1}{\left(\lambda-\mu_{1}\right)\left(\lambda-\mu_{2}\right)^{l}}\right. \\
& \left.Z_{2}\left(l, \lambda-\mu_{2}\right)-\frac{1}{\left(\mu_{1}-\mu_{2}\right)\left(\lambda-\mu_{1}\right)^{l}} Z_{1}\left(l, \lambda-\mu_{1}\right)-\frac{1}{\left(\lambda-\mu_{2}\right)^{l}} Z_{2}\left(l, \lambda-\mu_{2}\right)+\frac{1}{(l+1)\left(\lambda-\mu_{2}\right)} \frac{t^{l+2}}{l+2}\right) \\
& E\left(N_{3}(t)\left(N_{3}(t)-1\right)\right)=\sum_{l=2}^{\infty} l(l-1)\left[\frac{\lambda^{l} \mu_{1}^{l} \mu_{2} \mu_{3}}{(l-1) !\left(\lambda-\mu_{1}\right)^{2 l-1}\left(\mu_{3}-\mu_{2}\right)} \sum_{i=1}^{l}(-1)^{i+1} A_{i}\left(\lambda-\mu_{1}\right)^{l-i}\right. \\
& \left(Z_{2}\left(l-i, \mu_{1}-\mu_{2}\right)-Z_{3}\left(l-i, \mu_{1}-\mu_{3}\right)-(-1)^{l-1+i}\left(Z_{2}\left(l-i, \lambda-\mu_{2}\right)+Z_{3}\left(l-i, \lambda-\mu_{3}\right)\right)\right) \\
& -\frac{\lambda^{l+1} \mu_{1}^{l+1} \mu_{2} \mu_{3}}{l !\left(\lambda-\mu_{1}\right)^{2 l+1}\left(\mu_{3}-\mu_{2}\right)} \sum_{i=1}^{l+1}(-1)^{i+1} A_{i}\left(\lambda-\mu_{1}\right)^{l+1-i}\left(Z_{2}\left(l+1-i, \mu_{1}-\mu_{2}\right)-Z_{3}\left(l+1-i, \mu_{1}-\mu_{3}\right)\right. \\
& \left.-(-1)^{l+i}\left(Z_{2}\left(l+1-i, \lambda-\mu_{2}\right)+Z_{3}\left(l+1-i, \lambda-\mu_{3}\right)\right)\right) \\
& V\left(N_{3}(t)\right)=E\left(N_{3}(t)\left(N_{3}(t)-1\right)\right)+E\left(N_{3}(t)\right)-E\left(N_{3}(t)\right)^{2} \\
& E(t)=E\left(\frac{N_{3}(t)}{t}\right)=\frac{E\left(N_{3}(t)\right)}{t} \\
& V(t)=V\left(\frac{N_{3}(t)}{t}\right)=\frac{V\left(N_{3}(t)\right)}{t^{2}} \\
& E=\lim _{t \rightarrow \infty} E(t) \\
& V=\lim _{t \rightarrow \infty} V(t)
\end{aligned}
$$

where

$$
\begin{aligned}
& Z_{1}(x, y)=\frac{1}{y^{x}} \int_{0}^{t} \Gamma(x+1, y s) e^{-\mu_{1} s} \mathrm{ds} \\
& Z_{2}(x, y)=\frac{1}{y^{x}} \int_{0}^{t} \Gamma(x+1, y s) e^{-\mu_{2} s} \mathrm{~d} s
\end{aligned}
$$




\section{Case 3}

$$
\begin{aligned}
& E\left(N_{3}(t)\right)=\sum_{l=1}^{\infty} l\left[\frac { \lambda \mu _ { 1 } \mu _ { 2 } \mu _ { 3 } ^ { l } } { ( l - 1 ) ! ( \lambda - \mu _ { 1 } ) } \left(\frac{1}{\left(\mu_{1}-\mu_{2}\right)\left(\lambda-\mu_{2}\right)^{l-1}} Z_{2}\left(l-1, \lambda-\mu_{2}\right)-\frac{1}{\left(\mu_{1}-\mu_{2}\right)\left(\lambda-\mu_{1}\right)^{l-1}}\right.\right. \\
& \left.Z_{1}\left(l-1, \lambda-\mu_{1}\right)-\frac{1}{\left(\lambda-\mu_{2}\right)^{l}} Z_{2}\left(l-1, \lambda-\mu_{2}\right)+\frac{1}{l\left(\lambda-\mu_{2}\right)} \frac{t^{l+1}}{l+1}\right)-\frac{\lambda \mu_{1} \mu_{2} \mu_{3}^{l+1}}{l !\left(\lambda-\mu_{1}\right)}\left(\frac{1}{\left(\lambda-\mu_{1}\right)\left(\lambda-\mu_{2}\right)^{l}}\right. \\
& \left.Z_{2}\left(l, \lambda-\mu_{2}\right)-\frac{1}{\left(\mu_{1}-\mu_{2}\right)\left(\lambda-\mu_{1}\right)^{l}} Z_{1}\left(l, \lambda-\mu_{1}\right)-\frac{1}{\left(\lambda-\mu_{2}\right)^{l}} Z_{2}\left(l, \lambda-\mu_{2}\right)+\frac{1}{(l+1)\left(\lambda-\mu_{2}\right)} \frac{t^{l+2}}{l+2}\right) \\
& E\left(N_{3}(t)\left(N_{3}(t)-1\right)\right)=\sum_{l=2}^{\infty} l(l-1)\left[\frac{\lambda^{l} \mu_{1}^{l} \mu_{2} \mu_{3}}{(l-1) !\left(\lambda-\mu_{1}\right)^{2 l-1}\left(\mu_{3}-\mu_{2}\right)} \sum_{i=1}^{l}(-1)^{i+1} A_{i}\left(\lambda-\mu_{1}\right)^{l-i}\right. \\
& \left(Z_{2}\left(l-i, \mu_{1}-\mu_{2}\right)-Z_{3}\left(l-i, \mu_{1}-\mu_{3}\right)-(-1)^{l-1+i}\left(Z_{2}\left(l-i, \lambda-\mu_{2}\right)+Z_{3}\left(l-i, \lambda-\mu_{3}\right)\right)\right) \\
& -\frac{\lambda^{l+1} \mu_{1}^{l+1} \mu_{2} \mu_{3}}{l !\left(\lambda-\mu_{1}\right)^{2 l+1}\left(\mu_{3}-\mu_{2}\right)} \sum_{i=1}^{l+1}(-1)^{i+1} A_{i}\left(\lambda-\mu_{1}\right)^{l+1-i}\left(Z_{2}\left(l+1-i, \mu_{1}-\mu_{2}\right)-Z_{3}\left(l+1-i, \mu_{1}-\mu_{3}\right)\right. \\
& \left.-(-1)^{l+i}\left(Z_{2}\left(l+1-i, \lambda-\mu_{2}\right)+Z_{3}\left(l+1-i, \lambda-\mu_{3}\right)\right)\right) \\
& V\left(N_{3}(t)\right)=E\left(N_{3}(t)\left(N_{3}(t)-1\right)\right)+E\left(N_{3}(t)\right)-E\left(N_{3}(t)\right)^{2} \\
& E(t)=E\left(\frac{N_{3}(t)}{t}\right)=\frac{E\left(N_{3}(t)\right)}{t} \\
& V(t)=V\left(\frac{N_{3}(t)}{t}\right)=\frac{V\left(N_{3}(t)\right)}{t^{2}} \\
& E=\lim _{t \rightarrow \infty} E(t) \\
& V=\lim _{t \rightarrow \infty} V(t)
\end{aligned}
$$

where

$$
\begin{aligned}
& Z_{1}(x, y)=\frac{1}{y^{x}} \int_{0}^{t} \Gamma(x+1, y s) e^{-\mu_{1} s} \mathrm{ds} \\
& Z_{2}(x, y)=\frac{1}{y^{x}} \int_{0}^{t} \Gamma(x+1, y s) e^{-\mu_{2} s} \mathrm{~d} s
\end{aligned}
$$




\section{Case 4}

$$
\begin{aligned}
& E\left(N_{3}(t)\right)=\sum_{l=1}^{\infty} l\left[\frac { \lambda \mu _ { 1 } \mu _ { 2 } ^ { l } \mu _ { 3 } } { ( l - 1 ) ! ( \lambda - \mu _ { 1 } ) } \left(\frac{1}{\left(\mu_{1}-\mu_{3}\right)\left(\lambda-\mu_{3}\right)^{l-1}} Z_{3}\left(l-1, \lambda-\mu_{3}\right)-\frac{1}{\left(\mu_{1}-\mu_{3}\right)\left(\lambda-\mu_{1}\right)^{l-1}}\right.\right. \\
& Z_{1}\left(l-1, \lambda-\mu_{1}\right)-\frac{1}{\left(\lambda-\mu_{3}\right)^{l}} Z_{3}\left(l-1, \lambda-\mu_{3}\right)+\frac{t^{l+1}}{l(l+1)\left(\lambda-\mu_{3}\right)}-\frac{\lambda \mu_{1} \mu_{2}^{l+1} \mu_{3}}{l !\left(\lambda-\mu_{1}\right)}\left(\frac{1}{\left(\mu_{1}-\mu_{3}\right)\left(\lambda-\mu_{3}\right)^{l}}\right. \\
& \left.Z_{3}\left(l, \lambda-\mu_{3}\right)-\frac{1}{\left(\mu_{1}-\mu_{3}\right)\left(\lambda-\mu_{1}\right)^{l}} Z_{1}\left(l, \lambda-\mu_{1}\right)-\frac{1}{\left(\lambda-\mu_{3}\right)^{l+1}} Z_{3}\left(l, \lambda-\mu_{3}\right)+\frac{t^{l+2}}{(l+1)(l+2)\left(\lambda-\mu_{i}\right)}\right) \\
& E\left(N_{3}(t)\left(N_{3}(t)-1\right)\right)=\sum_{l=2}^{\infty} l(l-1)\left[\frac { \lambda \mu _ { 1 } \mu _ { 2 } ^ { l } \mu _ { 3 } } { ( l - 1 ) ! ( \lambda - \mu _ { 1 } ) } \left(\frac{1}{\left(\mu_{1}-\mu_{3}\right)\left(\lambda-\mu_{3}\right)^{l-1}} Z_{3}\left(l-1, \lambda-\mu_{3}\right)\right.\right. \\
& -\frac{1}{\left(\mu_{1}-\mu_{3}\right)\left(\lambda-\mu_{1}\right)^{l-1}} Z_{1}\left(l-1, \lambda-\mu_{1}\right)-\frac{1}{\left(\lambda-\mu_{3}\right)^{l}} Z_{3}\left(l-1, \lambda-\mu_{3}\right)+\frac{t^{l+1}}{l(l+1)\left(\lambda-\mu_{3}\right)}- \\
& \frac{\lambda \mu_{1} \mu_{2}^{l+1} \mu_{3}}{l !\left(\lambda-\mu_{1}\right)}\left(\frac{1}{\left(\mu_{1}-\mu_{3}\right)\left(\lambda-\mu_{3}\right)^{l}} Z_{3}\left(l, \lambda-\mu_{3}\right)-\frac{1}{\left(\mu_{1}-\mu_{3}\right)\left(\lambda-\mu_{1}\right)^{l}} Z_{1}\left(l, \lambda-\mu_{1}\right)-\right. \\
& \left.\frac{1}{\left(\lambda-\mu_{3}\right)^{l+1}} Z_{3}\left(l, \lambda-\mu_{3}\right)+\frac{t^{l+2}}{(l+1)(l+2)\left(\lambda-\mu_{3}\right)}\right) \\
& V\left(N_{3}(t)\right)=E\left(N_{3}(t)\left(N_{3}(t)-1\right)\right)+E\left(N_{3}(t)\right)-E\left(N_{3}(t)\right)^{2} \\
& E(t)=E\left(\frac{N_{3}(t)}{t}\right)=\frac{E\left(N_{3}(t)\right)}{t} \\
& V(t)=V\left(\frac{N_{3}(t)}{t}\right)=\frac{V\left(N_{3}(t)\right)}{t^{2}} \\
& E=\lim _{t \rightarrow \infty} E(t) \\
& V=\lim _{t \rightarrow \infty} V(t)
\end{aligned}
$$


APPENDIX A. TYPICAL SERIAL LINES: ANALYTICAL DERIVATIONS129

where

$$
\begin{aligned}
& Z_{1}(x, y)=\frac{1}{y^{x}} \int_{0}^{t} \Gamma(x+1, y s) e^{-\mu_{1} s} \mathrm{ds} \\
& Z_{3}(x, y)=\frac{1}{y^{x}} \int_{0}^{t} \Gamma(x+1, y s) e^{-\mu_{3} s} \mathrm{~d} s
\end{aligned}
$$




\section{A.4 Computational results}

\section{A.4.1 Atomic model}

Analytical results vs state-space representation

\begin{tabular}{|c|c|c|c|c|}
\hline$\left(\lambda, \mu_{1}\right)$ & \multicolumn{2}{|c|}{ Analytical Method } & \multicolumn{2}{c|}{ Markov Chain } \\
\hline \hline & Mean & Variance & Mean & Variance \\
\hline$(1,2)$ & 0.668 & 0.382 & 0.668 & 0.381 \\
\hline$(2,1)$ & 0.664 & 0.384 & 0.663 & 0.385 \\
\hline$(1,5)$ & 0.839 & 0.658 & 0.837 & 0.659 \\
\hline$(5,1)$ & 0.833 & 0.636 & 0.832 & 0.635 \\
\hline$(1,20)$ & 0.956 & 0.952 & 0.958 & 0.953 \\
\hline$(20,1)$ & 0.951 & 0.883 & 0.951 & 0.880 \\
\hline$(2,5)$ & 1.431 & 0.824 & 1.428 & 0.825 \\
\hline$(5,2)$ & 1.432 & 0.815 & 1.432 & 0.814 \\
\hline$(2,20)$ & 1.827 & 1.581 & 1.825 & 1.580 \\
\hline$(20,2)$ & 1.822 & 1.533 & 1.822 & 1.534 \\
\hline$(5,20)$ & 3.999 & 2.532 & 4.001 & 2.536 \\
\hline$(20,5)$ & 3.989 & 2.784 & 3.987 & 2.78 .5 \\
\hline
\end{tabular}

Table A.1: Analytical results vs state-space representations: the atomic model 


\section{Analytical results vs simulation}

\begin{tabular}{|c|c|c|c|c|}
\hline$(\lambda, \mu, t)$ & \multicolumn{2}{|c|}{ Analytical Model } & \multicolumn{2}{c|}{ Simulation Model } \\
\hline \hline & Mean & Variance & Mean & Variance \\
\hline$(1,2,2)$ & 0.890 & 0.374 & 0.882 & 0.383 \\
\hline$(1,2,5)$ & 0.732 & 0.372 & 0.744 & 0.373 \\
\hline$(1,2,10)$ & 0.654 & 0.374 & 0.641 & 0.372 \\
\hline$(1,2,25)$ & 0.685 & 0.393 & 0.686 & 0.391 \\
\hline$(1,2,50)$ & 0.678 & 0.383 & 0.675 & 0.385 \\
\hline$(1,2,100)$ & 0.666 & 0.384 & 0.668 & 0.384 \\
\hline
\end{tabular}
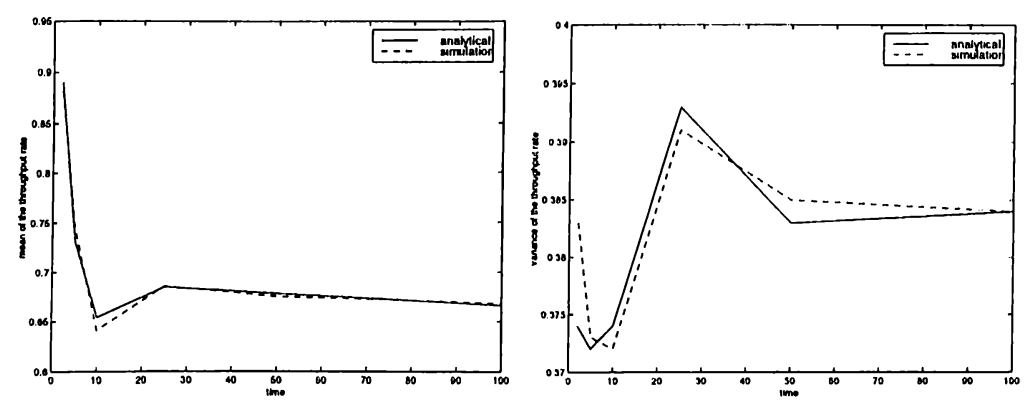

Figure A.4: Mean and Variance vs time: the atomic model

\begin{tabular}{|c|c|c|c|c|}
\hline$(\lambda, \mu, t)$ & \multicolumn{2}{|c|}{ Analytical Model } & \multicolumn{2}{c|}{ Simulation Model } \\
\hline \hline & Mean & Variance & Mean & Variance \\
\hline$(2,1,2)$ & 0.790 & 0.474 & 0.780 & 0.462 \\
\hline$(2,1,5)$ & 0.622 & 0.388 & 0.620 & 0.396 \\
\hline$(2,1,10)$ & 0.674 & 0.374 & 0.680 & 0.371 \\
\hline$(2,1,25)$ & 0.665 & 0.403 & 0.662 & 0.395 \\
\hline$(2,1,50)$ & 0.668 & 0.383 & 0.664 & 0.386 \\
\hline$(2,1,100)$ & 0.664 & 0.384 & 0.663 & 0.385 \\
\hline
\end{tabular}
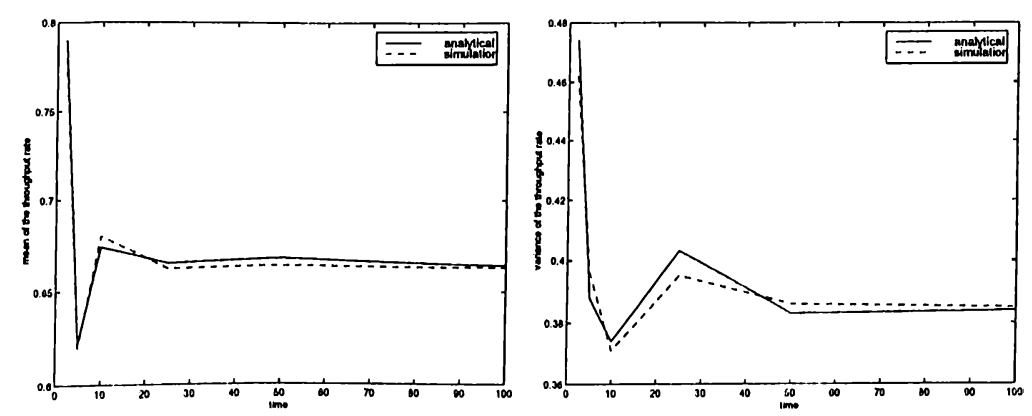

Table A.2: Mean and Variance vs time: the atomic model 


\begin{tabular}{|c|c|c|c|c|}
\hline$(\lambda, \mu, t)$ & \multicolumn{2}{|c|}{ Analytical Model } & \multicolumn{2}{c|}{ Simulation Model } \\
\hline \hline & Mean & Variance & Mean & Variance \\
\hline$(1,5,2)$ & 0.764 & 0.557 & 0.760 & 0.553 \\
\hline$(1,5,5)$ & 0.806 & 0.538 & 0.816 & 0.536 \\
\hline$(1,5,10)$ & 0.819 & 0.509 & 0.823 & 0.506 \\
\hline$(1,5,25)$ & 0.840 & 0.512 & 0.843 & 0.516 \\
\hline$(1,5,50)$ & 0.842 & 0.514 & 0.847 & 0.514 \\
\hline$(1,5,100)$ & 0.840 & 0.515 & 0.841 & 0.515 \\
\hline
\end{tabular}
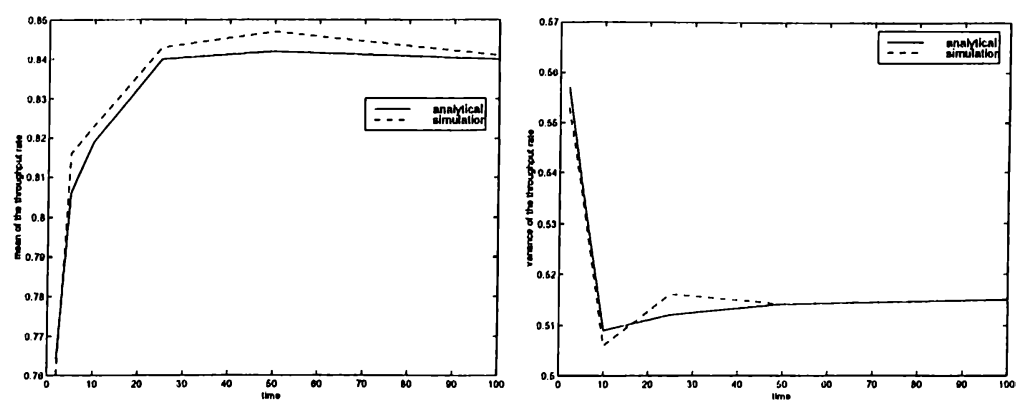

Table A.3: Mean and Variance vs time: the atomic model

\begin{tabular}{|c|c|c|c|c|}
\hline$(\lambda, \mu, t)$ & \multicolumn{2}{|c|}{ Analytical Model } & \multicolumn{2}{c|}{ Simulation Model } \\
\hline \hline & Mean & Variance & Mean & Variance \\
\hline$(2,20,2)$ & 1.798 & 0.736 & 1.803 & 0.731 \\
\hline$(2,20,5)$ & 1.812 & 1.388 & 1.808 & 1.486 \\
\hline$(2,20,10)$ & 1.892 & 1.496 & 1.896 & 1.501 \\
\hline$(2,20,25)$ & 1.842 & 1.450 & 1.841 & 1.451 \\
\hline$(2,20,50)$ & 1.834 & 1.451 & 1.831 & 1.449 \\
\hline$(2,20,100)$ & 1.829 & 1.449 & 1.827 & 1.449 \\
\hline
\end{tabular}
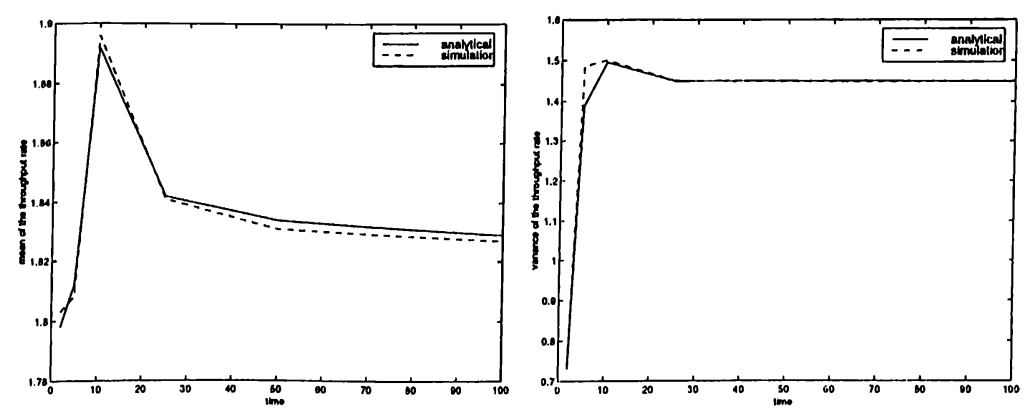

Table A.4: Mean and Variance vs time: the atomic model 
APPENDIX A. TYPICAL SERIAL LINES: ANALYTICAL DERIVATIONS133

A.4.2 Two-machine-one-buffer system

Analytical results vs state-space representation

\begin{tabular}{|c|c|c|c|c|}
\hline$\left(b_{1}, \lambda, \mu_{1}, \mu_{2}\right)$ & \multicolumn{2}{|c|}{ Analytical Method } & \multicolumn{2}{c|}{ Markov Chain } \\
\hline \hline & Mean & Variance & Mean & Variance \\
\hline$(0,2,3,3)$ & 0.970 & 0.361 & 0.971 & 0.361 \\
\hline$(0,5,3,3)$ & 1.289 & 0.487 & 1.286 & 0.483 \\
\hline$(0,20,3,3)$ & 1.473 & 0.741 & 1.474 & 0.739 \\
\hline$(1,1,3,3)$ & 0.738 & 0.431 & 0.739 & 0.434 \\
\hline$(1,2,3,3)$ & 1.137 & 0.481 & 1.137 & 0.480 \\
\hline$(1,5,3,3)$ & 1.619 & 0.608 & 1.620 & 0.608 \\
\hline$(1,20,3,3)$ & 1.919 & 1.008 & 1.921 & 1.011 \\
\hline$(5,2,3,3)$ & 1.199 & 0.581 & 1.196 & 0.582 \\
\hline$(5,5,3,3)$ & 1.843 & 0.992 & 1.845 & 0.991 \\
\hline$(5,20,3,3)$ & 2.391 & 1.230 & 2.389 & 1.229 \\
\hline$(0,2,7,3)$ & 1.122 & 0.478 & 1.124 & 0.479 \\
\hline$(0,5,7,3)$ & 1.640 & 0.681 & 1.639 & 0.682 \\
\hline$(0,20,7,3)$ & 2.025 & 1.112 & 2.027 & 1.114 \\
\hline$(1,2,7,3)$ & 1.394 & 0.641 & 1.393 & 0.641 \\
\hline$(1,5,7,3)$ & 2.124 & 0.895 & 2.123 & 0.894 \\
\hline$(1,20,7,3)$ & 2.574 & 1.583 & 2.575 & 1.584 \\
\hline$(5,2,7,3)$ & 1.541 & 0.966 & 1.540 & 0.967 \\
\hline$(5,5,7,3)$ & 2.609 & 1.164 & 2.605 & 1.163 \\
\hline$(5,20,7,3)$ & 2.965 & 2.512 & 2.966 & 2.510 \\
\hline$(0,2,3,7)$ & 1.121 & 0.515 & 1.123 & 0.518 \\
\hline$(0,5,3,7)$ & 1.641 & 0.686 & 1.640 & 0.685 \\
\hline$(0,20,3,7)$ & 2.024 & 1.163 & 2.020 & 1.161 \\
\hline$(1,2,3,7)$ & 1.194 & 0.575 & 1.194 & 0.576 \\
\hline$(1,5,3,7)$ & 1.840 & 0.856 & 1.841 & 0.856 \\
\hline$(1,20,3,7)$ & 2.430 & 1.635 & 2.432 & 1.631 \\
\hline$(5,2,3,7)$ & 1.200 & 0.582 & 1.199 & 0.579 \\
\hline$(5,5,3,7)$ & 1.872 & 0.971 & 1.878 & 0.974 \\
\hline$(5,20,3,7)$ & 2.596 & 0.814 & 2.597 & 0.815 \\
\hline
\end{tabular}

Table A.5: Analytical results vs state-space representations: the two-machine model 


\section{Analytical results vs simulation}

\begin{tabular}{|c|c|c|c|c|}
\hline$\left(b, \lambda, \mu_{1}, \mu_{2}, t\right)$ & \multicolumn{2}{|c|}{ Analytical Model } & \multicolumn{2}{c|}{ Simulation Model } \\
\hline \hline & Mean & Variance & Mean & Variance \\
\hline$(0,1,3,3,2)$ & 0.780 & 0.271 & 0.798 & 0.263 \\
\hline$(0,1,3,3,5)$ & 0.730 & 0.287 & 0.712 & 0.281 \\
\hline$(0,1,3,3,10)$ & 0.683 & 0.291 & 0.688 & 0.298 \\
\hline$(0,1,3,3,25)$ & 0.667 & 0.296 & 0.669 & 0.295 \\
\hline$(0,1,3,3,50)$ & 0.662 & 0.293 & 0.664 & 0.294 \\
\hline$(0,1,3,3,100)$ & 0.665 & 0.295 & 0.665 & 0.293 \\
\hline
\end{tabular}
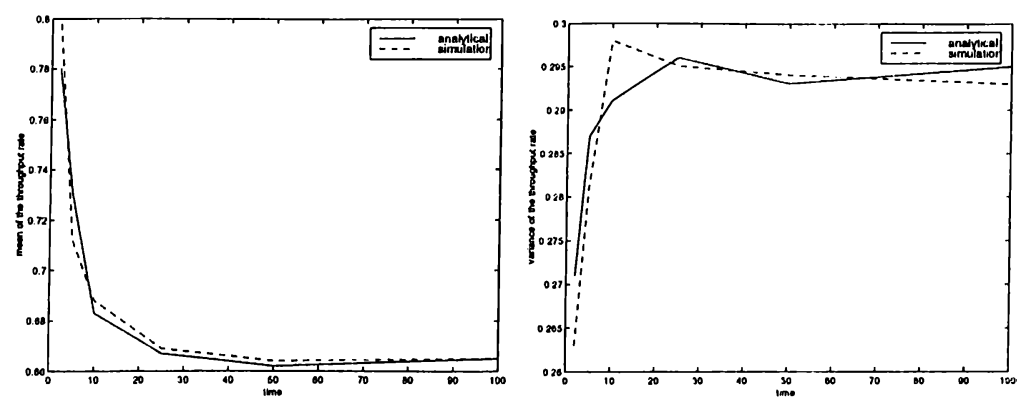

Table A.6: Mean and Variance vs time: the two-machine model

\begin{tabular}{|c|c|c|c|c|}
\hline$\left(b, \lambda, \mu_{1}, \mu_{2}, t\right)$ & \multicolumn{2}{|c|}{ Analytical Model } & \multicolumn{2}{c|}{ Simulation Model } \\
\hline \hline & Mean & Variance & Mean & Variance \\
\hline$(5,1,3,3,2)$ & 0.869 & 0.318 & 0.872 & 0.320 \\
\hline$(5,1,3,3,5)$ & 0.801 & 0.368 & 0.794 & 0.371 \\
\hline$(5,1,3,3,10)$ & 0.772 & 0.413 & 0.776 & 0.420 \\
\hline$(5,1,3,3,25)$ & 0.761 & 0.432 & 0.762 & 0.433 \\
\hline$(5,1,3,3,50)$ & 0.755 & 0.461 & 0.752 & 0.464 \\
\hline$(5,1,3,3,100)$ & 0.753 & 0.481 & 0.752 & 0.480 \\
\hline
\end{tabular}
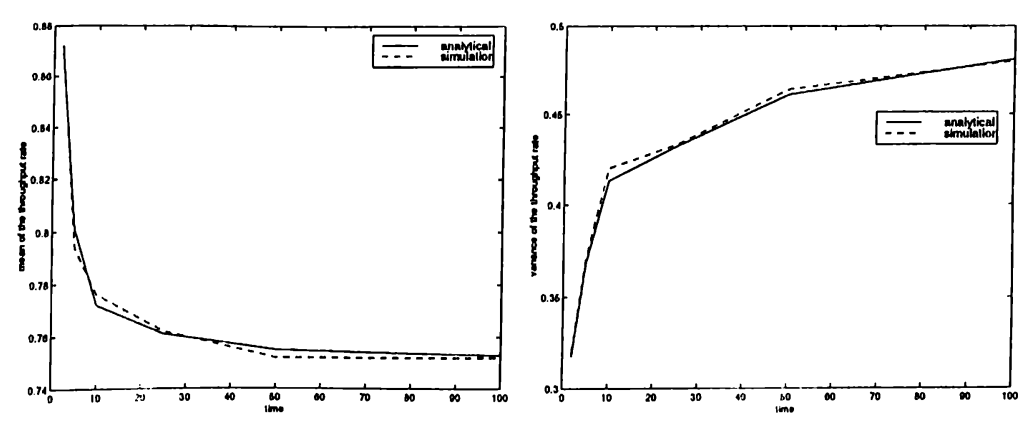

Table A.7: Mean and Variance vs time: the two-machine model 


\begin{tabular}{|c|c|c|c|c|}
\hline$\left(b, \lambda, \mu_{1}, \mu_{2}, t\right)$ & \multicolumn{2}{|c|}{ Analytical Model } & \multicolumn{2}{c|}{ Simulation Model } \\
\hline \hline & Mean & Variance & Mean & Variance \\
\hline$(0,1,7,3,2)$ & 0.918 & 0.397 & 0.921 & 0.395 \\
\hline$(0,1,7,3,5)$ & 0.802 & 0.405 & 0.805 & 0.403 \\
\hline$(0,1,7,3,10)$ & 0.767 & 0.401 & 0.771 & 0.403 \\
\hline$(0,1,7,3,25)$ & 0.742 & 0.391 & 0.744 & 0.390 \\
\hline$(0,1,7,3,50)$ & 0.728 & 0.400 & 0.729 & 0.398 \\
\hline$(0,1,7,3,100)$ & 0.725 & 0.423 & 0.725 & 0.422 \\
\hline
\end{tabular}
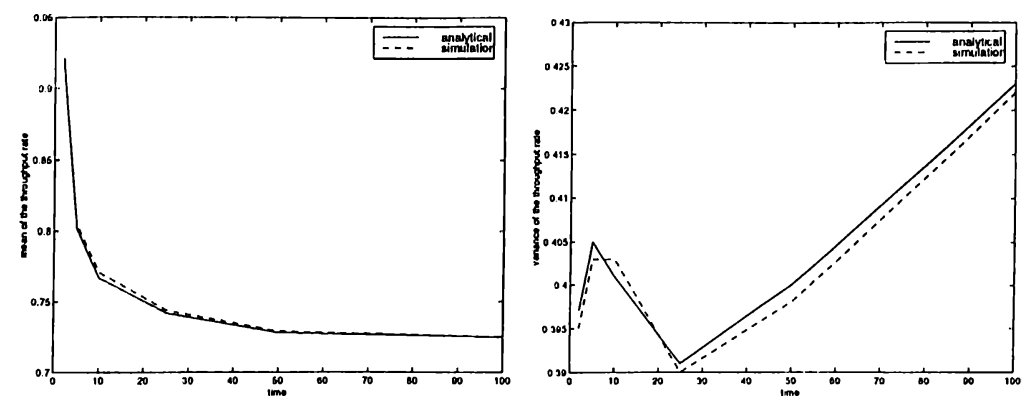

Table A.8: Mean and Variance vs time: the two-machine model

\begin{tabular}{|c|c|c|c|c|}
\hline$\left(b, \lambda, \mu_{1}, \mu_{2}, t\right)$ & \multicolumn{2}{|c|}{ Analytical Model } & \multicolumn{2}{c|}{ Simulation Model } \\
\hline \hline & Mean & Variance & Mean & Variance \\
\hline$(5,1,7,3,2)$ & 0.982 & 0.602 & 1.042 & 0.623 \\
\hline$(5,1,7,3,5)$ & 0.968 & 0.616 & 0.971 & 0.618 \\
\hline$(5,1,7,3,10)$ & 0.932 & 0.627 & 0.933 & 0.625 \\
\hline$(5,1,7,3,25)$ & 0.902 & 0.638 & 0.891 & 0.638 \\
\hline$(5,1,7,3,50)$ & 0.884 & 0.659 & 0.886 & 0.656 \\
\hline$(5,1,7,3,100)$ & 0.878 & 0.688 & 0.879 & 0.691 \\
\hline
\end{tabular}
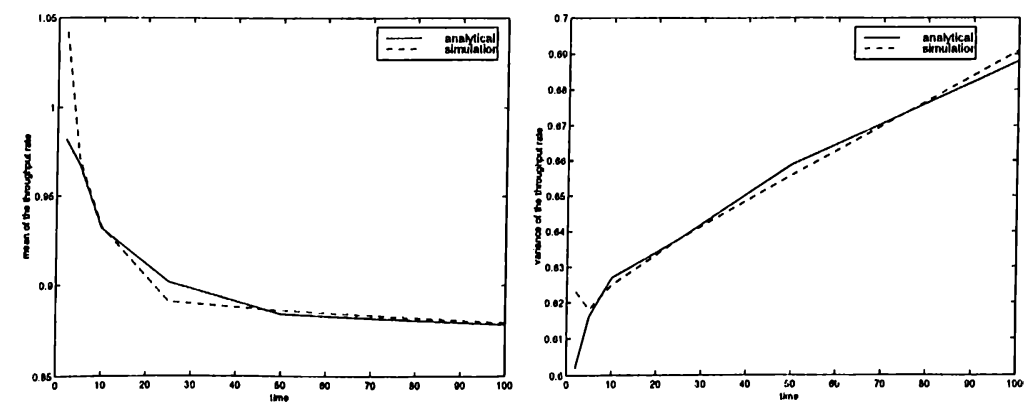

Table A.9: Mean and Variance vs time: the two-machine model 
APPENDIX A. TYPICAL SERIAL LINES: ANALYTICAL DERIVATIONS136

\section{A.4.3 Three-machine-two-buffer system}

\section{Analytical results vs state-space representation}

\begin{tabular}{|c|c|c|c|c|}
\hline$\left(b_{1}, b_{2}, \lambda, \mu_{1}, \mu_{2}, \mu_{3}\right)$ & \multicolumn{2}{|c|}{ Analytical Method } & \multicolumn{2}{c|}{ Markov Chain } \\
\hline \hline & Mean & Variance & Mean & Variance \\
\hline$(2,5,1,3,3,3)$ & 0.747 & 0.465 & 0.748 & 0.466 \\
\hline$(5,2,1,3,3,3)$ & 0.744 & 0.420 & 0.745 & 0.421 \\
\hline$(5,5,1,3,3,3)$ & 0.750 & 0.461 & 0.749 & 0.460 \\
\hline$(5,20,1,3,3,3)$ & 0.748 & 0.462 & 0.749 & 0.461 \\
\hline$(20,5,1,3,3,3)$ & 0.749 & 0.462 & 0.749 & 0.462 \\
\hline$(20,20,1,3,3,3)$ & 0.751 & 0.464 & 0.749 & 0.463 \\
\hline$(2,5,20,3,4,6)$ & 2.351 & 1.459 & 2.349 & 1.458 \\
\hline$(5,2,20,3,4,6)$ & 2.436 & 1.466 & 2.439 & 1.466 \\
\hline$(5,5,20,3,4,6)$ & 2.531 & 1.608 & 2.534 & 1.607 \\
\hline$(5,20,20,3,4,6)$ & 2.536 & 1.625 & 2.539 & 1.623 \\
\hline$(20,5,20,3,4,6)$ & 2.574 & 1.842 & 2.572 & 1.841 \\
\hline$(20,20,20,3,4,6)$ & 2.574 & 1.901 & 2.575 & 1.900 \\
\hline$(2,5,20,4,3,6)$ & 2.424 & 1.249 & 2.425 & 1.249 \\
\hline$(5,5,20,4,3,6)$ & 2.706 & 1.552 & 2.707 & 1.550 \\
\hline$(5,20,20,4,3,6)$ & 2.717 & 1.782 & 2.717 & 1.783 \\
\hline$(20,5,20,4,3,6)$ & 2.884 & 2.221 & 2.884 & 2.223 \\
\hline$(20,20,20,4,3,6)$ & 2.899 & 2.096 & 2.898 & 2.095 \\
\hline$(2,5,20,6,4,3)$ & 2.725 & 1.651 & 2.723 & 1.654 \\
\hline$(5,5,20,6,4,3)$ & 2.798 & 1.846 & 2.797 & 1.848 \\
\hline$(5,20,20,6,4,3)$ & 2.947 & 2.687 & 2.947 & 2.686 \\
\hline$(20,5,20,6,4,3)$ & 2.832 & 2.294 & 2.829 & 2.290 \\
\hline$(20,20,20,6,4,3)$ & 2.946 & 3.025 & 2.947 & 3.023 \\
\hline
\end{tabular}

Table A.10: Analytical results vs state-space representations: the threemachine model 


\section{Analytical results vs simulation}

\begin{tabular}{|c|c|c|c|c|}
\hline$\left(b, \lambda, \mu_{1}, \mu_{2}, t\right)$ & \multicolumn{2}{|c|}{ Analytical Model } & \multicolumn{2}{c|}{ Simulation Model } \\
\hline \hline & Mean & Variance & Mean & Variance \\
\hline$(2,2,20,6,4,3,2)$ & 1.856 & 1.277 & 1.769 & 1.281 \\
\hline$(2,2,20,6,4,3,5)$ & 2.168 & 1.346 & 2.164 & 1.425 \\
\hline$(2,2,20,6,4,3,10)$ & 2.305 & 1.371 & 2.302 & 1.377 \\
\hline$(2,2,20,6,4,3,25)$ & 2.382 & 1.387 & 2.378 & 1.390 \\
\hline$(2,2,20,6,4,3,50)$ & 2.402 & 1.392 & 2.398 & 1.394 \\
\hline$(2,2,20,6,4,3,100)$ & 2.417 & 1.406 & 2.416 & 1.402 \\
\hline
\end{tabular}
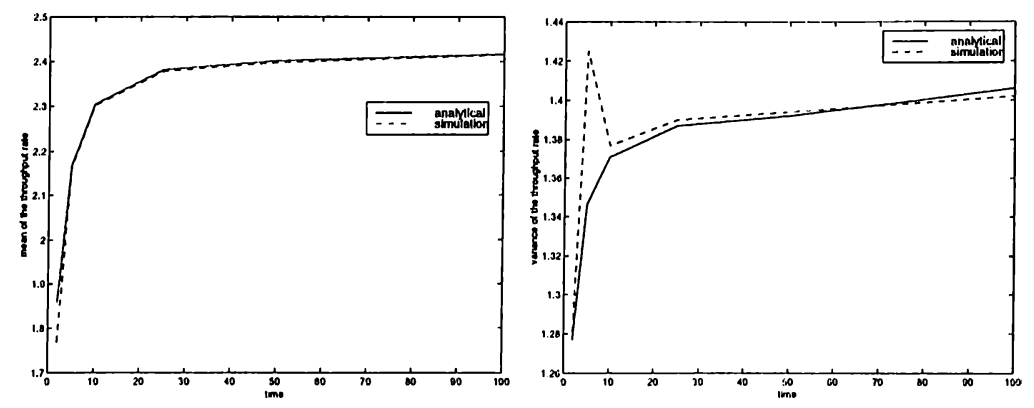

Table A.11: Mean and Variance vs time: the three-machine model

\begin{tabular}{|c|c|c|c|c|}
\hline$\left(b, \lambda, \mu_{1}, \mu_{2}, t\right)$ & \multicolumn{2}{|c|}{ Analytical Model } & \multicolumn{2}{c|}{ Simulation Model } \\
\hline \hline & Mean & Variance & Mean & Variance \\
\hline$(2,5,20,6,4,3,2)$ & 2.012 & 1.364 & 1.767 & 1.361 \\
\hline$(2,5,20,6,4,3,5)$ & 2.296 & 1.492 & 2.287 & 1.467 \\
\hline$(2,5,20,6,4,3,10)$ & 2.511 & 1.668 & 2.509 & 1.666 \\
\hline$(2,5,20,6,4,3,25)$ & 2.674 & 1.692 & 2.666 & 1.688 \\
\hline$(2,5,20,6,4,3,50)$ & 2.703 & 1.715 & 2.699 & 1.716 \\
\hline$(2,5,20,6,4,3,100)$ & 2.723 & 1.728 & 2.723 & 1.727 \\
\hline
\end{tabular}
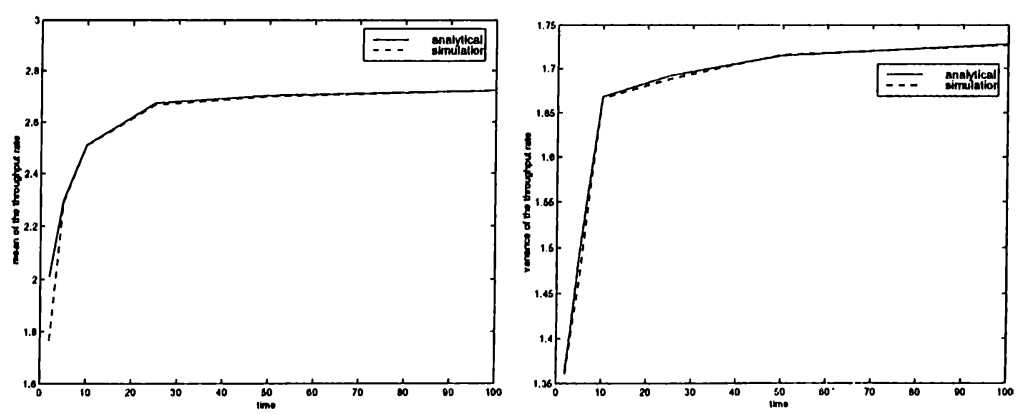

Table A.12: Mean and Variance vs time: the three-machine model 


\begin{tabular}{|c|c|c|c|c|}
\hline$\left(b, \lambda, \mu_{1}, \mu_{2}, t\right)$ & \multicolumn{2}{|c|}{ Analytical Model } & \multicolumn{2}{c|}{ Simulation Model } \\
\hline \hline & Mean & Variance & Mean & Variance \\
\hline$(5,2,20,6,4,3,2)$ & 1.872 & 1.312 & 1.695 & 1.257 \\
\hline$(5,2,20,6,4,3,5)$ & 2.268 & 1.348 & 2.160 & 1.350 \\
\hline$(5,2,20,6,4,3,10)$ & 2.322 & 1.398 & 2.318 & 1.408 \\
\hline$(5,2,20,6,4,3,25)$ & 2.436 & 1.418 & 2.428 & 1.422 \\
\hline$(5,2,20,6,4,3,50)$ & 2.453 & 1.424 & 2.454 & 1.428 \\
\hline$(5,2,20,6,4,3,100)$ & 2.472 & 1.432 & 2.473 & 1.431 \\
\hline
\end{tabular}
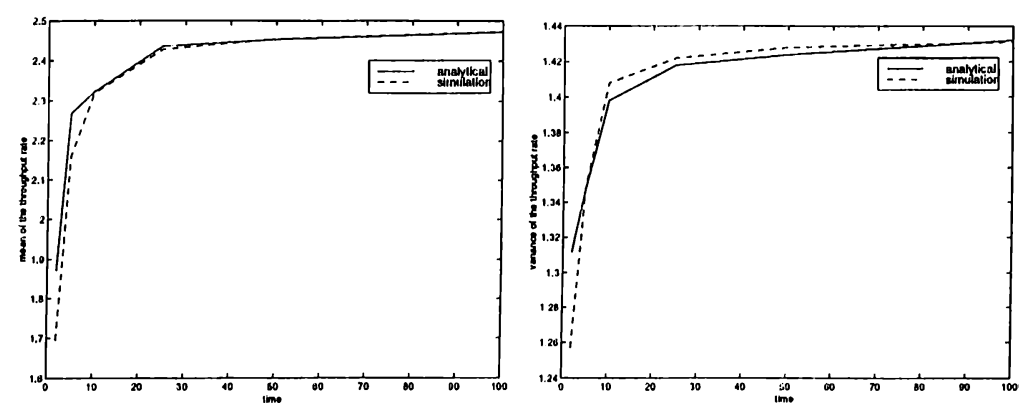

Table A.13: Mean and Variance vs time: the three-machine model

\begin{tabular}{|c|c|c|c|c|}
\hline$\left(b, \lambda, \mu_{1}, \mu_{2}, t\right)$ & \multicolumn{2}{|c|}{ Analytical Model } & \multicolumn{2}{c|}{ Simulation Model } \\
\hline \hline & Mean & Variance & Mean & Variance \\
\hline$(5,5,20,6,4,3,2)$ & 1.735 & 1.402 & 1.733 & 1.395 \\
\hline$(5,5,20,6,4,3,5)$ & 2.319 & 1.662 & 2.320 & 1.665 \\
\hline$(5,5,20,6,4,3,10)$ & 2.528 & 1.713 & 2.523 & 1.628 \\
\hline$(5,5,20,6,4,3,25)$ & 2.720 & 1.798 & 2.713 & 1.803 \\
\hline$(5,5,20,6,4,3,50)$ & 2.771 & 1.814 & 2.767 & 1.811 \\
\hline$(5,5,20,6,4,3,100)$ & 2.976 & 1.847 & 2.997 & 1.848 \\
\hline
\end{tabular}
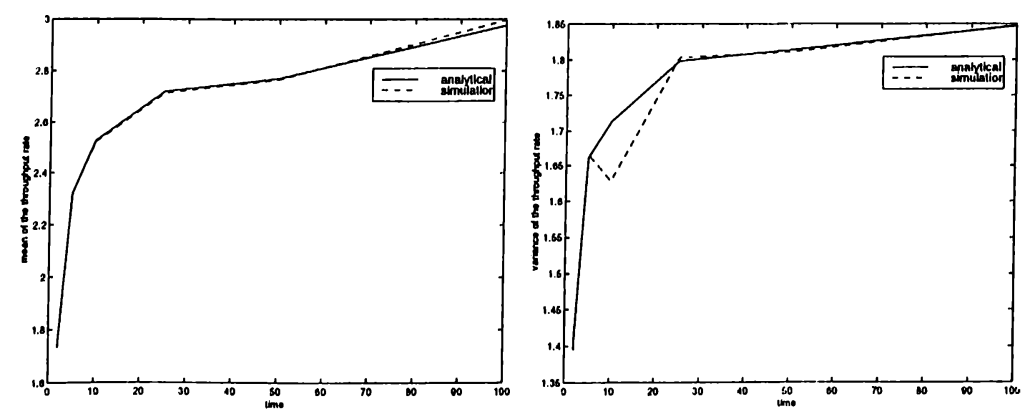

Table A.14: Mean and Variance vs time: the three-machine model 


\section{Appendix B}

\section{Two-part type systems: Analytical derivations}

\section{B.1 Two-machine-one-buffer system}

Case $1: T_{\lambda}^{j, i}+T_{\mu_{1}}^{j, i} \geq T_{\mu_{2}}^{j, i-1}$ holds for each part $i$ of type $j$.

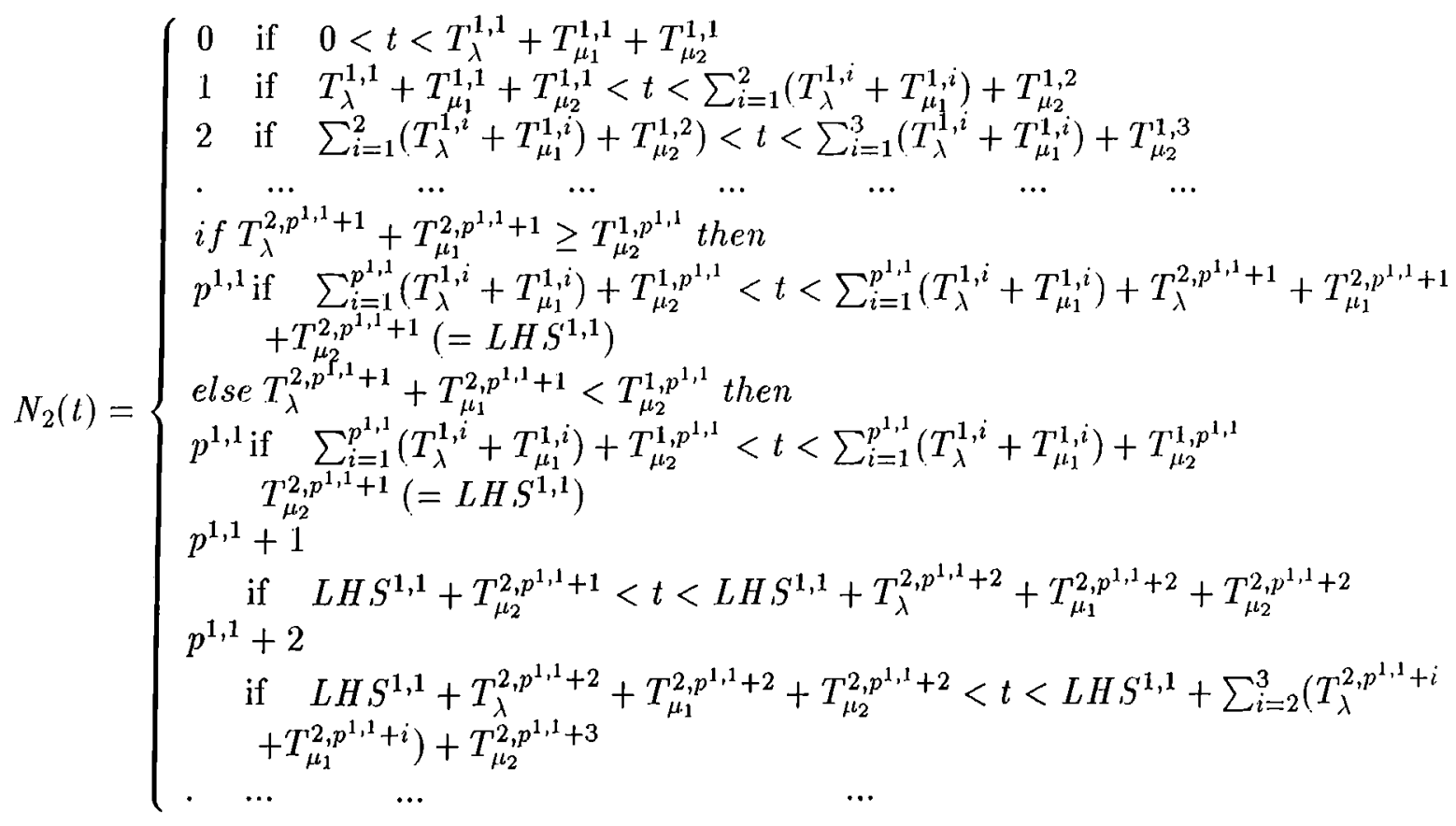




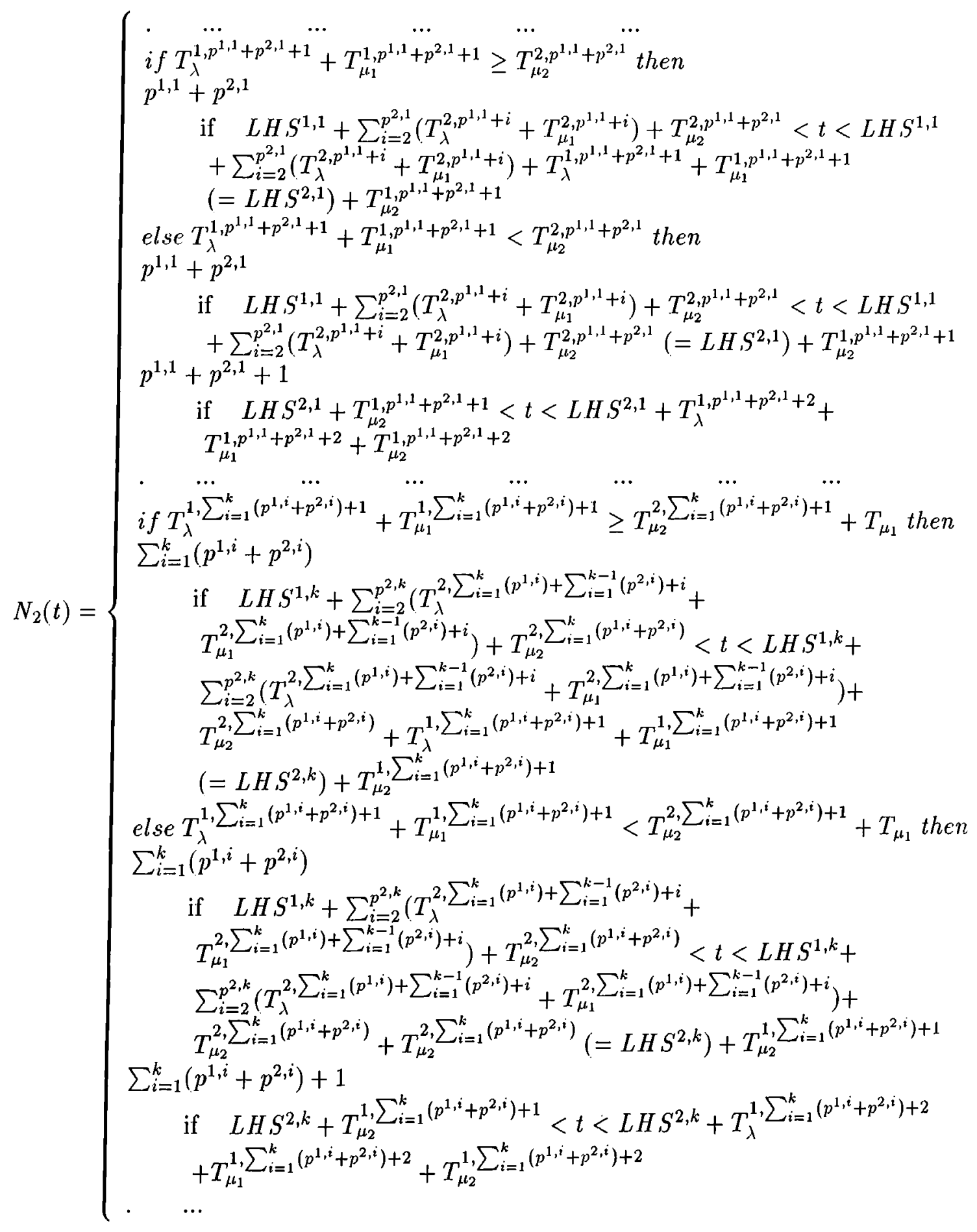


Case 2: $T_{\lambda}^{j, i}+T_{\mu_{1}}^{j, i}<T_{\mu_{2}}^{j, i-1}$ holds for each part $i$ of type $j$.

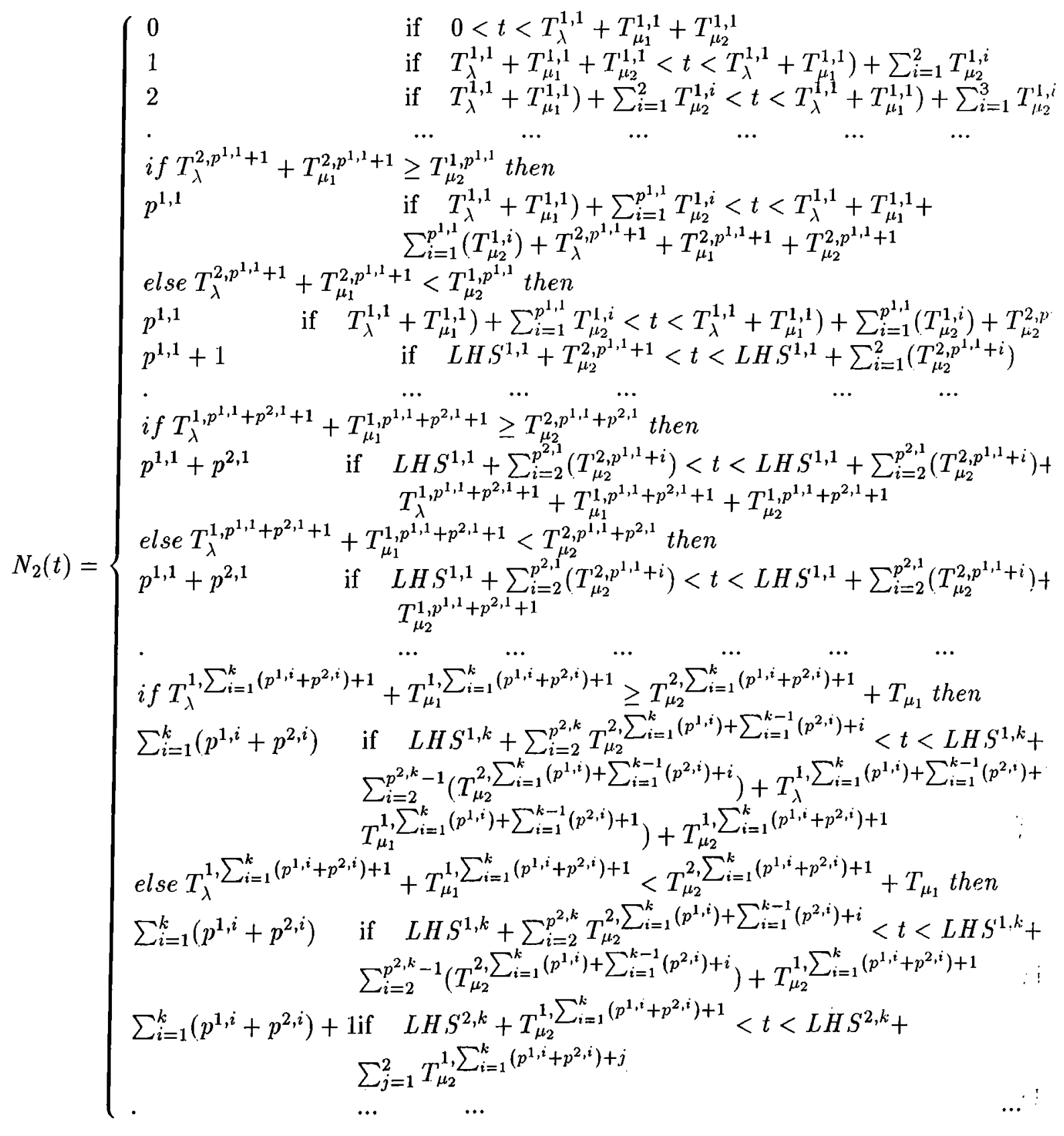


where

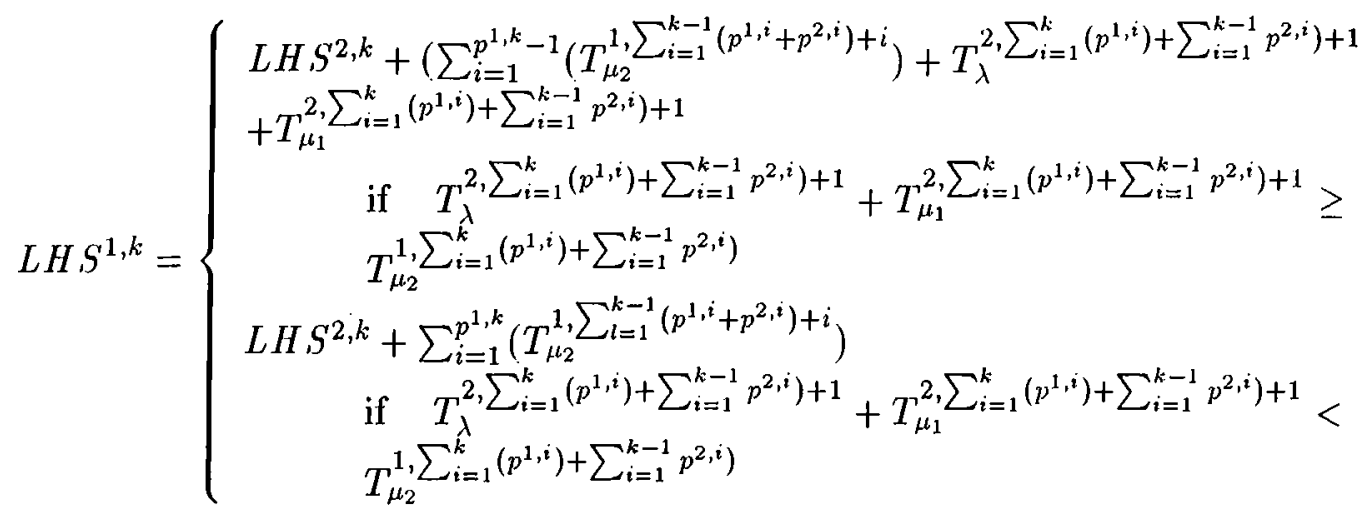

and

$$
L H S^{2, k}=\left\{\begin{array}{l}
L H S^{1, k}+\left(\sum_{i=1}^{p^{2, k}-1}\left(T_{\mu_{2}}^{2, \sum_{i=1}^{k}\left(p^{1, i}\right)+\sum_{i=1}^{k-1}\left(p^{2, i}\right)+i}\right)+T_{\lambda}^{1, \sum_{i=1}^{k}\left(p^{1, i}+p^{2, i}\right)+1}+\right. \\
T_{\mu_{1}}^{1, \sum_{i=1}^{k}\left(p^{1, i}+p^{2, i}\right)+1}+ \\
\quad \text { if } T_{\lambda}^{1, \sum_{i=1}^{k}\left(p^{1, i}+p^{2, i}\right)+1}+T_{\mu_{1}}^{1, \sum_{i=1}^{k}\left(p^{1, i}+p^{2, i}\right)+1} \geq T_{\mu_{2}}^{2, \sum_{i=1}^{k}\left(p^{1, i}+p^{2, i}\right)} \\
L H S^{1, k}+\sum_{i=1}^{p^{2, k}}\left(T_{\mu_{2}}^{2, \sum_{i=1}^{k}\left(p^{1, i}\right)+\sum_{i=1}^{k-1}\left(p^{2, i}\right)+i}\right) \\
\text { if } T_{\lambda}^{1, \sum_{i=1}^{k}\left(p^{1, i}+p^{2, i}\right)+1}+T_{\mu_{1}}^{1, \sum_{i=1}^{k}\left(p^{1, i}+p^{2, i}\right)+1} \geq T_{\mu_{2}}^{2, \sum_{i=1}^{k}\left(p^{1, i}+p^{2, i}\right)}
\end{array}\right.
$$




\section{B.2 Three-machine-two-buffer system}

Case 1: $T_{\lambda}^{j, i}+T_{\mu_{1}}^{j, i} \geq T_{\mu_{2}}^{j, i-1}$ and $T_{\lambda}^{j, i}+T_{\mu_{1}}^{j, i}+T_{\mu_{2}}^{j, i} \geq T_{\mu_{2}}^{j, i-1}+T_{\mu_{3}}^{j, i-1}$ hold for each part $i$ of type $j$.

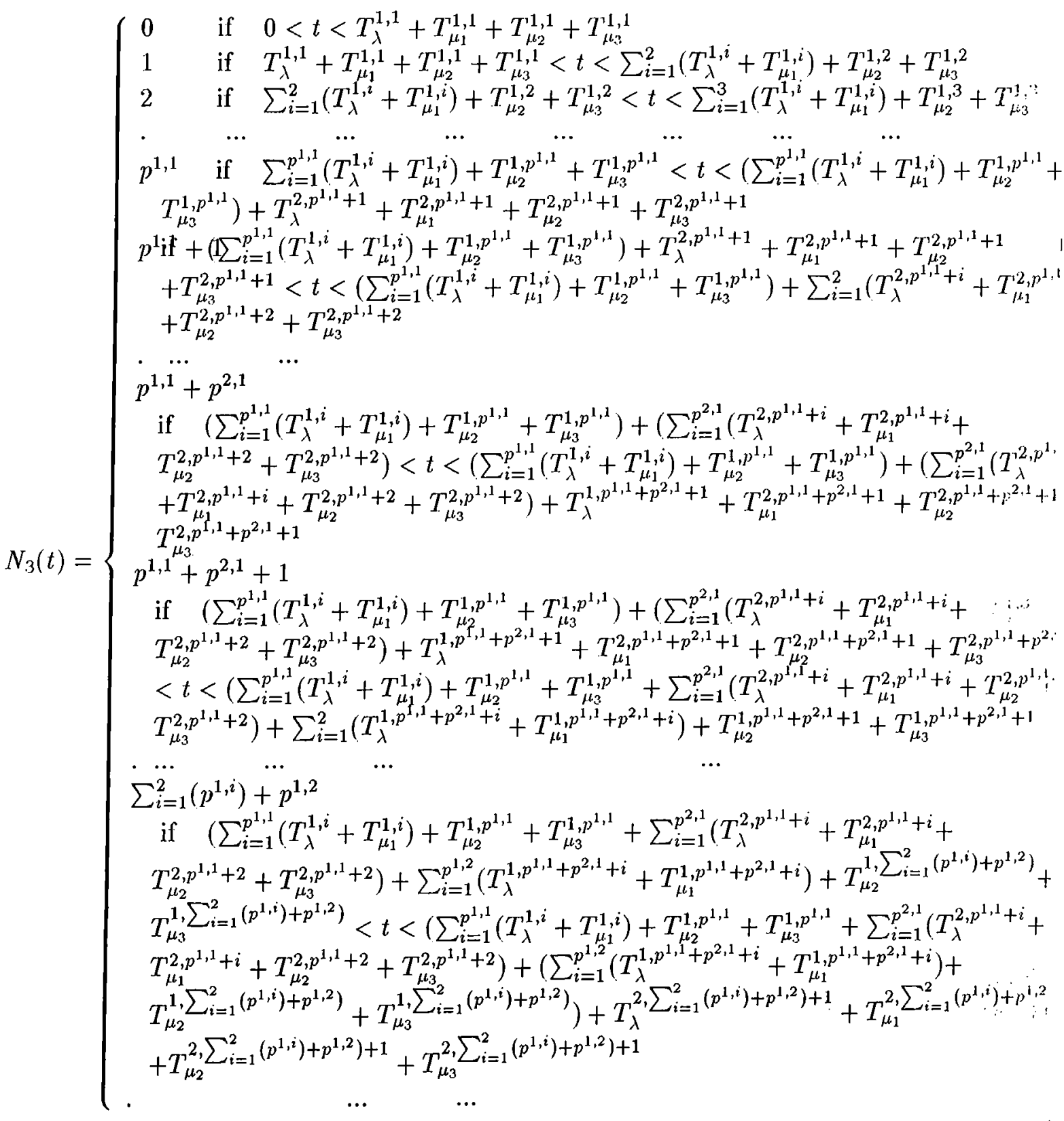




$$
\begin{aligned}
& \int \dot{\sum}_{i=1}^{2}\left(p^{1, i}+p^{2, i}\right) \\
& \text { if } \quad\left(\sum_{i=1}^{p^{1,1}}\left(T_{\lambda}^{1, i}+T_{\mu_{1}}^{1, i}\right)+T_{\mu_{2}}^{1, p^{1,1}}+T_{\mu_{3}}^{1, p^{1,1}}+\sum_{i=1}^{p^{2,1}}\left(T_{\lambda}^{2, p^{1,1}+i}+T_{\mu_{1}, p^{1,1}+i}+\right.\right. \\
& \left.T_{\mu_{2}}^{2, p^{1,1}+p^{2,1}}+T_{\mu_{3}}^{2, p^{1,1}+p^{2,1}}\right)+\left(\sum_{i=1}^{p^{1,2}}\left(T_{\lambda}^{1, p^{1,1}+p^{2,1}+i}+T_{\mu_{1}}^{1, p^{1,1}+p^{2,1}+i}\right)+\right. \\
& T_{\mu_{2}}^{\left.1, \sum_{i=1}^{2}\left(p^{1, i}\right)+p^{2,1}\right)}+T_{\mu_{3}}^{\left.1, \sum_{i=1}^{2}\left(p^{1, i}\right)+p^{2,1}\right)}+\left(\sum _ { i = 1 } ^ { p ^ { 2 , 2 } } \left(T_{\lambda}^{\left.2, \sum_{i=1}^{2}\left(p^{1, i}\right)+p^{2,1}\right)+i}+\right.\right. \\
& \left.\left.T_{\mu_{1}}^{\left.2, \sum_{i=1}^{2}\left(p^{1, i}\right)+p^{2,1}\right)+i}\right)+T_{\mu_{2}}^{2, \sum_{i=1}^{2}\left(p^{1, i}+p^{2, i}\right)}+T_{\mu_{3}}^{2, \sum_{i=1}^{2}\left(p^{1, i}+p^{2, i}\right)}\right)<t< \\
& \left(\sum_{i=1}^{p^{1,1}}\left(T_{\lambda}^{1, i}+T_{\mu_{1}}^{1, i}\right)+T_{\mu_{2}}^{1, p^{1,1}}+T_{\mu_{3}}^{1, p^{1,1}}+\sum_{i=1}^{p^{2,1}}\left(T_{\lambda}^{2, p^{1,1}+i}+T_{\mu_{1}}^{2, p^{1,1}+i}+T_{\mu_{2}}^{2, p^{1,1}+p^{2,1}}-\right.\right. \\
& \left.T_{\mu_{3}}^{2, p^{1,1}+p^{2,1}}\right)+\left(\sum_{i=1}^{p^{1,2}}\left(T_{\lambda}^{1, p^{1,1}+p^{2,1}+i}+T_{\mu_{1}}^{1, p^{1,1}+p^{2,1}+i}\right)+T_{\mu_{2}}^{\left.1, \sum_{i=1}^{2}\left(p^{1, i}\right)+p^{2,1}\right)}+\right. \\
& T_{\mu_{3}}^{\left.1, \sum_{i=1}^{2}\left(p^{1, i}\right)+p^{2,1}\right)}+\sum_{i=1}^{p^{2,2}}\left(T_{\lambda}^{\left.2, \sum_{i=1}^{2}\left(p^{1, i}\right)+p^{2,1}\right)+i}+T_{\mu_{1}}^{\left.2, \sum_{i=1}^{2}\left(p^{1, i}\right)+p^{2,1}\right)+i}\right)+ \\
& \left.T_{\mu_{2}}^{2, \sum_{i=1}^{2}\left(p^{1, i}+p^{2, i}\right)}+T_{\mu_{3}}^{2, \sum_{i=1}^{2}\left(p^{1, i}+p^{2, i}\right)}\right)+T_{\lambda}^{1, \sum_{i=1}^{2}\left(p^{1, i}+p^{2, i}\right)+1}+T_{\mu_{1}}^{1, \sum_{i=1}^{2}\left(p^{1, i}+p^{2, i}\right)+1} \\
& T_{\mu_{2}}^{1, \sum_{i=1}^{2}\left(p^{1, i}+p^{2, i}\right)+1}+T_{\mu_{3}}^{1, \sum_{i=1}^{2}\left(p^{1, i}+p^{2, i}\right)+1} \\
& \sum_{i=1}^{k}\left(p^{1, i}+p^{2, i}\right) \\
& \text { if } \sum_{i=1}^{k}\left(\sum_{j=1}^{p^{1, i}}\left(T_{\lambda}^{1, \sum_{l=1}^{i-1}\left(p^{1, l}+p^{2, l}\right)+j}+T_{\mu_{1}}^{1, \sum_{l=1}^{i-1}\left(p^{1, l}+p^{2, l}\right)+j}\right)+\right. \\
& T_{\mu_{2}}^{1, \sum_{l=1}^{i-1}\left(p^{1, l}+p^{2, l}\right)+p^{1, i}}+T_{\mu_{3}}^{1, \sum_{l=1}^{i-1}\left(p^{1, l}+p^{2, l}\right)+p^{1, i}}+ \\
& \sum_{j=1}^{p^{2, i}}\left(T_{\lambda}^{2, \sum_{l=1}^{i}\left(p^{1, l}\right)+\sum_{l=1}^{i-1}\left(p^{2, l}\right)+j}+T_{\mu_{1}}^{2, \sum_{l=1}^{i}\left(p^{1, l}\right)+\sum_{l=1}^{i-1}\left(p^{2, l}\right)+j}+\right. \\
& \left.T_{\mu_{2}}^{2, \sum_{l=1}^{i}\left(p^{1, l}+p^{2, l}\right)}+T_{\mu_{3}}^{2, \sum_{l=1}^{i}\left(p^{1, l}+p^{2, l}\right)}\right)<t<\sum_{i=1}^{k}\left(\sum _ { j = 1 } ^ { p ^ { 1 , i } } \left(T_{\lambda}^{1, \sum_{l=1}^{i-1}\left(p^{1, l}+p^{2, l}\right)+j}\right.\right. \\
& \left.+T_{\mu_{1}}^{1, \sum_{l=1}^{i-1}\left(p^{1, l}+p^{2, l}\right)+j}\right)+T_{\mu_{2}}^{1, \sum_{l=1}^{i-1}\left(p^{1, l}+p^{2, l}\right)+p^{1, i}}+T_{\mu_{3}}^{1, \sum_{l=1}^{i-1}\left(p^{1, l}+p^{2, l}\right)+p^{1, i}}+
\end{aligned}
$$

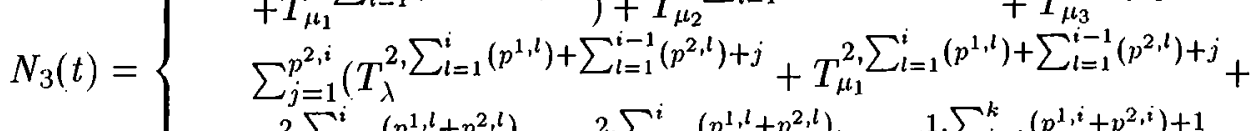

$$
\begin{aligned}
& \left.T_{\mu_{2}}^{2, \sum_{l=1}^{i}\left(p^{1, l}+p^{2, l}\right)}+T_{\mu_{3}}^{2, \sum_{l=1}^{i}\left(p^{1, l}+p^{2, l}\right)}\right)+T_{\lambda}^{1, \sum_{i=1}^{k}\left(p^{1, i}+p^{2, i}\right)+1}+ \\
& T_{\mu_{1}}^{1, \sum_{i=1}^{k}\left(p^{1, i}+p^{2, i}\right)+1}+T_{\mu_{2}}^{1, \sum_{i=1}^{k}\left(p^{1, i}+p^{2, i}\right)+1}+T_{\mu_{3}}^{1, \sum_{i=1}^{k}\left(p^{1, i}+p^{2, i}\right)+1} \\
& \sum_{i=1}^{k+1}\left(p^{1, i}\right)+\sum_{i=1}^{k}\left(p^{2, i}\right) \\
& \text { if } \quad\left(\sum _ { i = 1 } ^ { k } \left(\sum_{j=1}^{p^{1, i}}\left(T_{\lambda}^{1, \sum_{l=1}^{i-1}\left(p^{1, l}+p^{2, l}\right)+j}+T_{\mu_{1}}^{1, \sum_{l=1}^{i-1}\left(p^{1, l}+p^{2, l}\right)+j}\right)+\right.\right. \\
& T_{\mu_{2}}^{1, \sum_{l=1}^{i-1}\left(p^{1, l}+p^{2, l}\right)+p^{1, i}}+T_{\mu_{3}}^{1, \sum_{l=1}^{i-1}\left(p^{1, l}+p^{2, l}\right)+p^{1, i}}+ \\
& \sum_{j=1}^{p^{2, i}}\left(T_{\lambda}^{2, \sum_{l=1}^{i}\left(p^{1, l}\right)+\sum_{l=1}^{i-1}\left(p^{2, l}\right)+j}+T_{\mu_{1}}^{2, \sum_{l=1}^{i}\left(p^{1, l}\right)+\sum_{l=1}^{i-1}\left(p^{2, l}\right)+i}+\right. \\
& \left.\left.T_{\mu_{2}}^{2, \sum_{l=1}^{i}\left(p^{1, l}+p^{2, l}\right)}+T_{\mu_{3}}^{2, \sum_{l=1}^{i}\left(p^{1, l}+p^{2, l}\right)}\right)\right)+\sum_{i=1}^{p^{1, k+1}}\left(T_{\lambda}^{1, \sum_{i=1}^{k}\left(p^{1, i}+p^{2, i}\right)+i}+\right. \\
& \left.T_{\mu_{1}}^{1, \sum_{i=1}^{k}\left(p^{1, i}+p^{2, i}\right)+i}\right)+T_{\mu_{2}}^{1, \sum_{i=1}^{k+1}\left(p^{1, i}\right)+\sum_{i=1}^{k}\left(p^{2, i}\right)}+T_{\mu_{3}}^{1, \sum_{i=1}^{k+1}\left(p^{1, i}\right)+\sum_{i=1}^{k}\left(p^{2, i}\right)} \\
& <t<\left(\sum _ { i = 1 } ^ { k } \left(\sum_{j=1}^{p^{1, i}}\left(T_{\lambda}^{1, \sum_{l=1}^{i-1}\left(p^{1, l}+p^{2, l}\right)+j}+T_{\mu_{1}}^{1, \sum_{l=1}^{i-1}\left(p^{1, l}+p^{2, l}\right)+j}\right)+\right.\right. \\
& T_{\mu_{2}}^{1, \sum_{l=1}^{i-1}\left(p^{1, l}+p^{2, l}\right)+p^{1, i}}+T_{\mu_{3}}^{1, \sum_{l=1}^{i-1}\left(p^{1, l}+p^{2, l}\right)+p^{1, i}}+ \\
& \sum_{j=1}^{p^{2, i}}\left(T_{\lambda}^{2, \sum_{l=1}^{i}\left(p^{1, l}\right)+\sum_{l=1}^{i-1}\left(p^{2, l}\right)+j}+T_{\mu_{1}}^{2, \sum_{l=1}^{i}\left(p^{1, l}\right)+\sum_{l=1}^{i-1}\left(p^{2, l}\right)+j}+\right. \\
& \left.\left.T_{\mu_{2}}^{2, \sum_{l=1}^{i}\left(p^{1, l}+p^{2, l}\right)}+T_{\mu_{3}}^{2, \sum_{l=1}^{i}\left(p^{1, l}+p^{2, l}\right)}\right)\right)+\sum_{i=1}^{p^{1, k+1}}\left(T_{\lambda}^{1, \sum_{i=1}^{k}\left(p^{1, i}+p^{2, i}\right)+i}+\right. \\
& \left.T_{\mu_{1}}^{1, \sum_{i=1}^{k}\left(p^{1, i}+p^{2, i}\right)+i}\right)+T_{\mu_{2}}^{1, \sum_{i=1}^{k+1}\left(p^{1, i}\right)+\sum_{i=1}^{k}\left(p^{2, i}\right)}+T_{\mu_{3}}^{1, \sum_{i=1}^{k+1}\left(p^{1, i}\right)+\sum_{i=1}^{k}\left(p^{2, i}\right)}+ \\
& T_{\lambda}^{2, \sum_{i=1}^{k+1}\left(p^{1, i}\right)+\sum_{i=1}^{k}\left(p^{2, i}\right)+1}+T_{\mu_{1}}^{2, \sum_{i=1}^{k+1}\left(p^{1, i}\right)+\sum_{i=1}^{k}\left(p^{2, i}\right)+1}+ \\
& T_{\mu_{2}}^{2, \sum_{i=1}^{k+1}\left(p^{1, i}\right)+\sum_{i=1}^{k}\left(p^{2, i}\right)+1}+T_{\mu_{3}}^{2, \sum_{i=1}^{k+1}\left(p^{1, i}\right)+\sum_{i=1}^{k}\left(p^{2, i}\right)+1}
\end{aligned}
$$




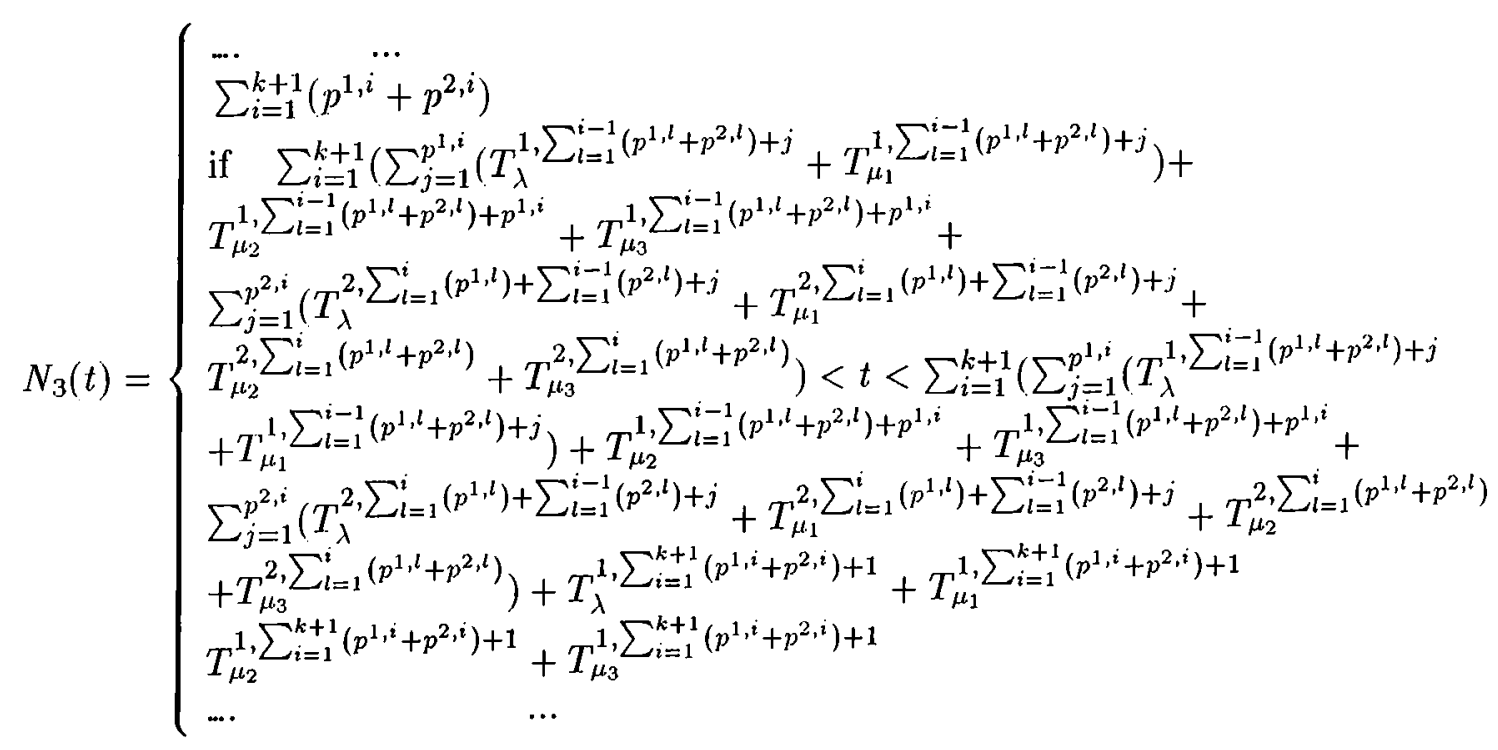

Case 2: $T_{\lambda}^{j, i}+T_{\mu_{1}}^{j, i} \geq T_{\mu_{2}}^{j, i-1}$ and $T_{\lambda}^{j, i}+T_{\mu_{1}}^{j, i}+T_{\mu_{2}}^{j, i}<T_{\mu_{2}}^{j, i-1}+T_{\mu_{3}}^{j, i-1}$ hold for each part $i$ of type $j$.

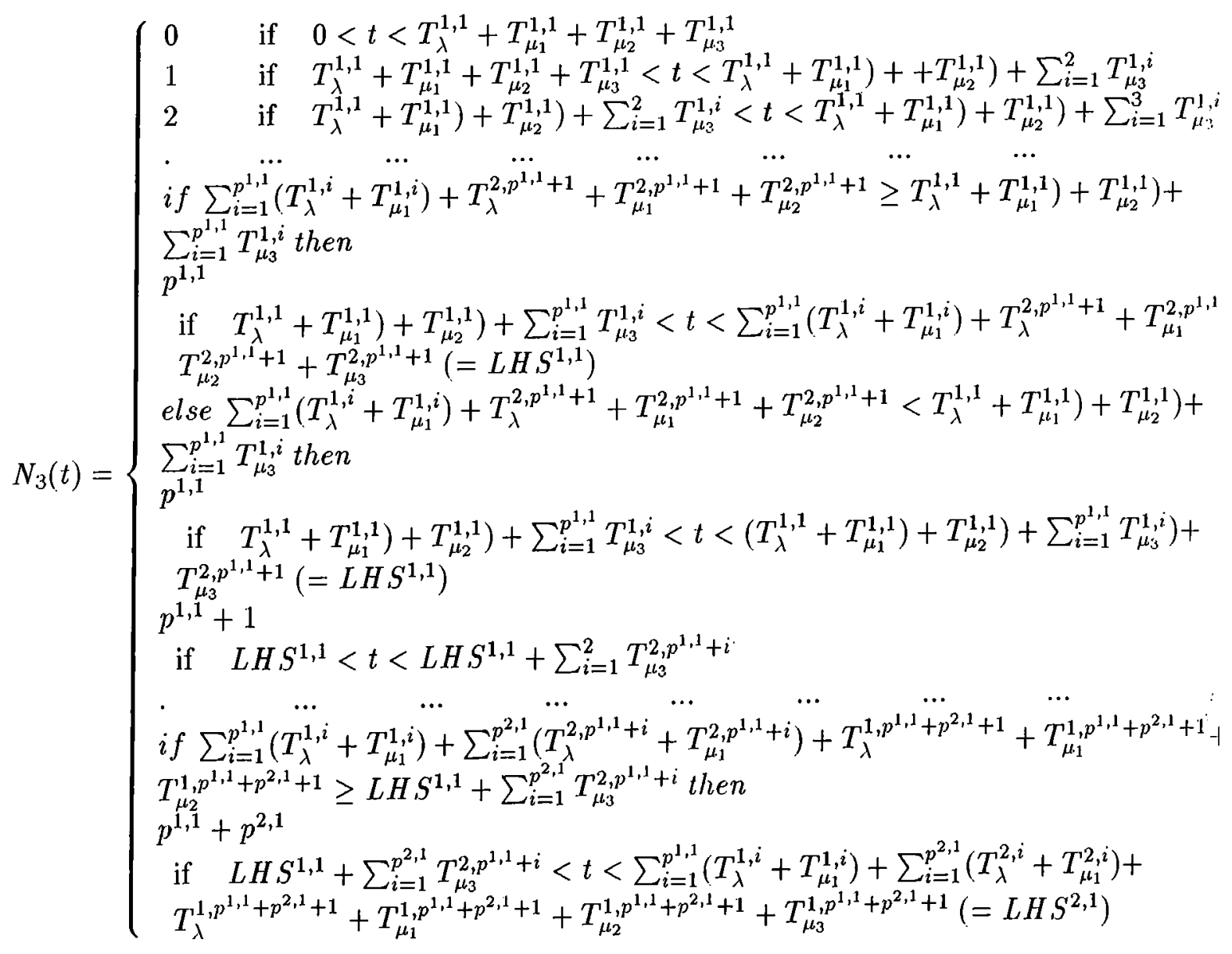




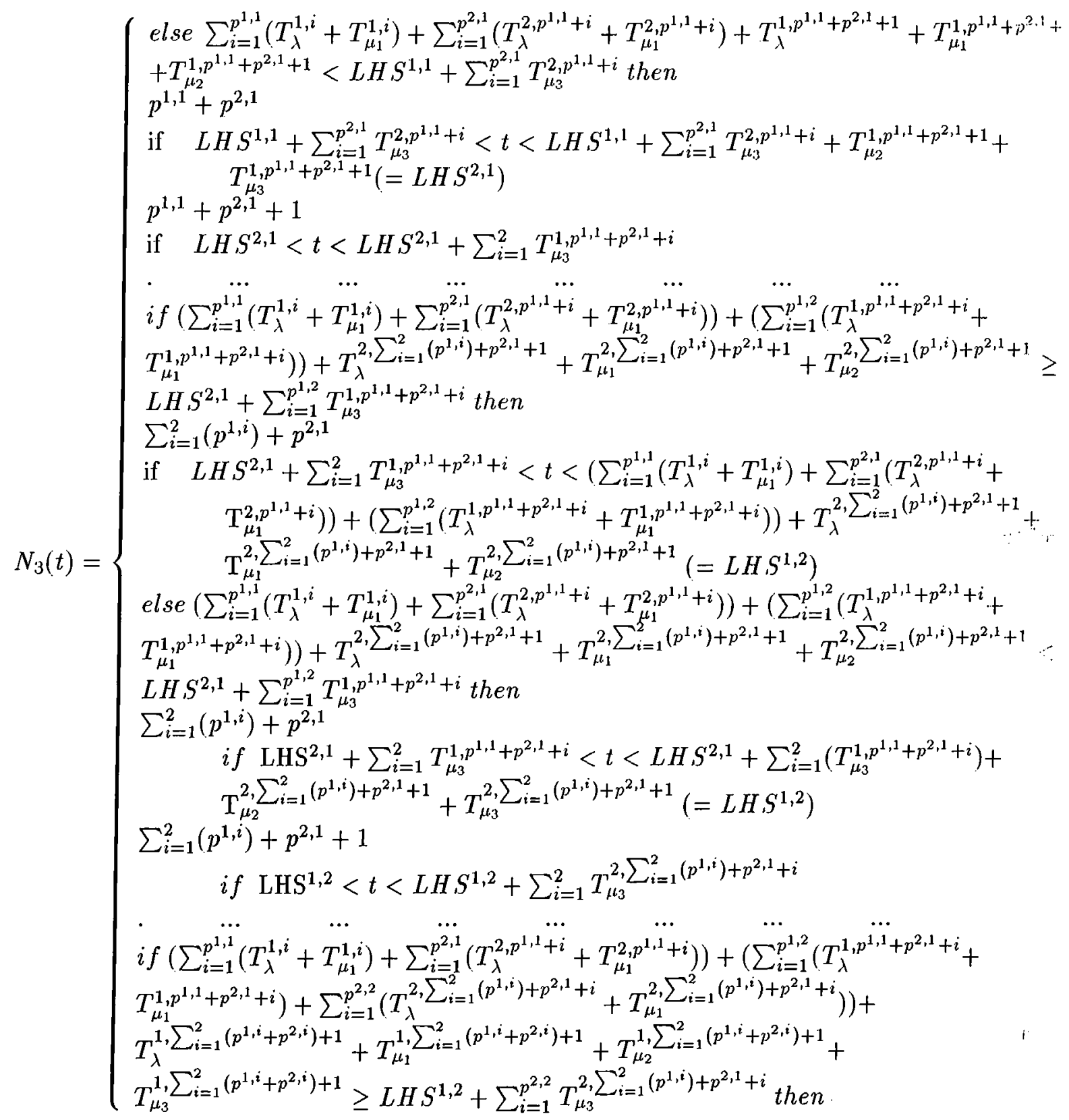




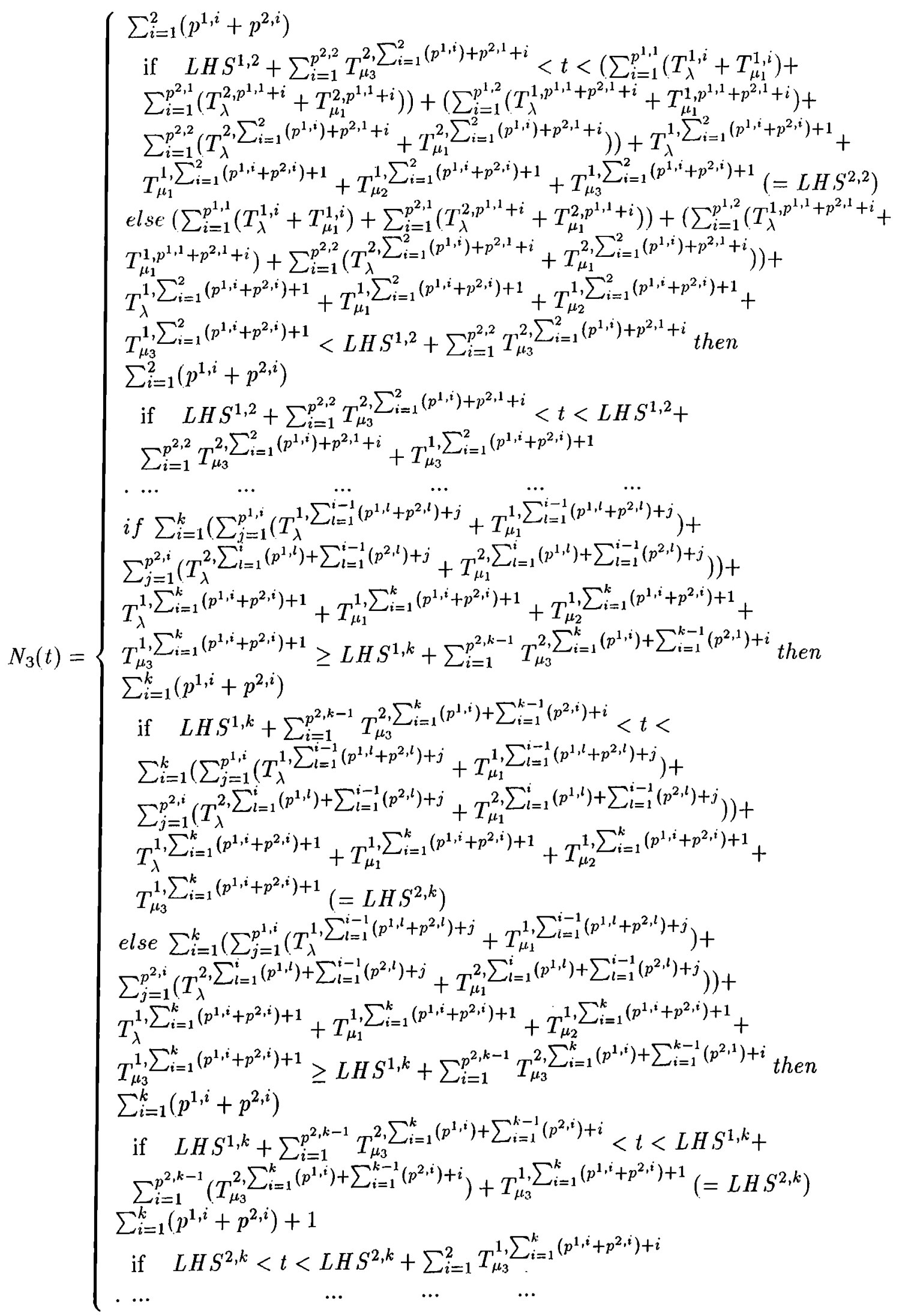




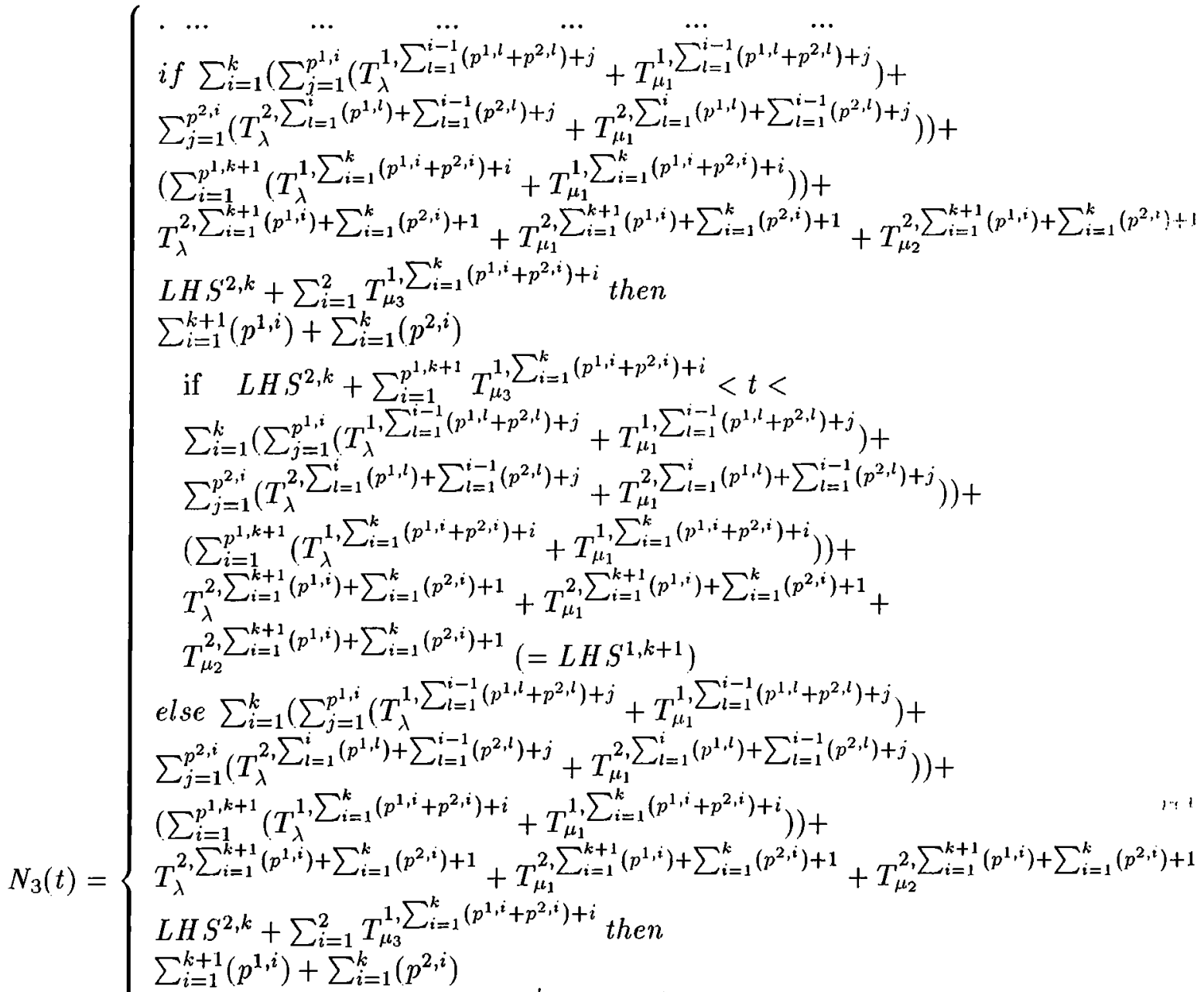

$$
\begin{aligned}
& \text { if } L H S^{2, k}+\sum_{i=1}^{p^{1, k+1}} T_{\mu_{3}}^{1, \sum_{i=1}^{k}\left(p^{1, i}+p^{2, i}\right)+i}<t<L H S^{2, k}+ \\
& \sum_{i=1}^{p^{1, k+1}}\left(T_{\mu_{3}}^{1, \sum_{i=1}^{k}\left(p^{1, i}+p^{2, i}\right)+i}\right)+T_{\mu_{3}}^{2, \sum_{i=1}^{k+1}\left(p^{1, i}\right)+\sum_{i=1}^{k}\left(p^{2, i}\right)+1}\left(=\text { LH } S^{1, k+1}\right) \\
& \sum_{i=1}^{k+1}\left(p^{1, i}\right)+\sum_{i=1}^{k}\left(p^{2, i}\right)+1 \\
& \text { if } L H S^{1, k+1}<t<L H S^{1, k+1}+\sum_{i=1}^{2} T_{\mu_{3}}^{2, \sum_{i=1}^{k+1}\left(p^{1, i}\right)+\sum_{i=1}^{k}\left(p^{2, i}\right)+i}
\end{aligned}
$$$$
\text { if } \sum_{i=1}^{k+1}\left(\sum_{j=1}^{p^{1, i}}\left(T_{\lambda}^{1, \sum_{l=1}^{i-1}\left(p^{1, l}+p^{2, l}\right)+j}+T_{\mu_{1}}^{1, \sum_{l=1}^{i-1}\left(p^{1, l}+p^{2, l}\right)+j}\right)+\right.
$$$$
\left.\sum_{j=1}^{p^{2, i}}\left(T_{\lambda}^{2, \sum_{l=1}^{i}\left(p^{1, l}\right)+\sum_{l=1}^{i-1}\left(p^{2, l}\right)+j}+T_{\mu_{1}}^{2, \sum_{l=1}^{i}\left(p^{1, l}\right)+\sum_{l=1}^{i-1}\left(p^{2, l}\right)+j}\right)\right)+
$$$$
T_{\lambda}^{1, \sum_{i=1}^{k+1}\left(p^{1, i}+p^{2, i}\right)+1}+T_{\mu_{1}}^{1, \sum_{i=1}^{k+1}\left(p^{1, i}+p^{2, i}\right)+1}+T_{\mu_{2}}^{1, \sum_{i=1}^{k+1}\left(p^{1, i}+p^{2, i}\right)+1}+
$$$$
T_{\mu_{3}}^{1, \sum_{i=1}^{k+1}\left(p^{1, i}+p^{2, i}\right)+1} \geq \text { LHS } S^{1, k+1}+\sum_{i=1}^{p^{2, k}} T_{\mu_{3}}^{2, \sum_{i=1}^{k+1}\left(p^{1, i}\right)+\sum_{i=1}^{k}\left(p^{2,1}\right)+i} \text { then }
$$$$
\sum_{i=1}^{k+1}\left(p^{1, i}+p^{2, i}\right)
$$$$
\text { if } L H S^{1, k+1}+\sum_{i=1}^{p^{2, k}} T_{\mu_{3}}^{2, \sum_{i=1}^{k+1}\left(p^{1, i}\right)+\sum_{i=1}^{k}\left(p^{2, i}\right)+i}<t<
$$$$
\sum_{i=1}^{k+1}\left(\sum_{j=1}^{p^{1, i}}\left(T_{\lambda}^{1, \sum_{l=1}^{i-1}\left(p^{1, l}+p^{2, l}\right)+j}+T_{\mu_{1}}^{1, \sum_{l=1}^{i-1}\left(p^{1, l}+p^{2, l}\right)+j}\right)+\right.
$$$$
\left.\sum_{j=1}^{p^{2, i}}\left(T_{\lambda}^{2, \sum_{l=1}^{i}\left(p^{1, l}\right)+\sum_{l=1}^{i-1}\left(p^{2, l}\right)+j}+T_{\mu_{1}}^{2, \sum_{l=1}^{i}\left(p^{1, l}\right)+\sum_{l=1}^{i-1}\left(p^{2, l}\right)+j}\right)\right)+
$$$$
T_{\lambda}^{1, \sum_{i=1}^{k+1}\left(p^{1, i}+p^{2, i}\right)+1}+T_{\mu_{1}}^{1, \sum_{i=1}^{k+1}\left(p^{1, i}+p^{2, i}\right)+1}+T_{\mu_{2}}^{1, \sum_{i=1}^{k+1}\left(p^{1, i}+p^{2, i}\right)+1}+
$$$$
T_{\mu_{3}}^{1, \sum_{i=1}^{k+1}\left(p^{1, i}+p^{2, i}\right)+1}\left(=L H S^{2, k+1}\right)
$$ 


$$
N_{3}(t)=\left\{\begin{array}{l}
\text { else } \sum_{i=1}^{k+1}\left(\sum_{j=1}^{p^{1, i}}\left(T_{\lambda}^{1, \sum_{l=1}^{i-1}\left(p^{1, l}+p^{2, l}\right)+j}+T_{\mu_{1}}^{1, \sum_{l=1}^{i-1}\left(p^{1, l}+p^{2, l}\right)+j}\right)+\right. \\
\left.\sum_{j=1}^{p^{2, i}}\left(T_{\lambda}^{2, \sum_{l=1}^{i}\left(p^{1, i}\right)+\sum_{l=1}^{i-1}\left(p^{2, l}\right)+j}+T_{\mu_{1}}^{2, \sum_{l=1}^{i}\left(p^{1, l}\right)+\sum_{l=1}^{i-1}\left(p^{2, l}\right)+j}\right)\right)+ \\
T_{\lambda}^{1, \sum_{i=1}^{k+1}\left(p^{1, i}+p^{2, i}\right)+1}+T_{\mu_{1}}^{1, \sum_{i=1}^{k+1}\left(p^{1, i}+p^{2, i}\right)+1}+T_{\mu_{2}}^{1, \sum_{i=1}^{k+1}\left(p^{1, i}+p^{2, i}\right)+1}+ \\
T_{\mu_{3}}^{1, \sum_{i=1}^{k+1}\left(p^{1, i}+p^{2, i}\right)+1}<L H S^{1, k+1}+\sum_{i=1}^{p^{2, k}} T_{\mu_{3}}^{2, \sum_{i=1}^{k+1}\left(p^{1, i}\right)+\sum_{i=1}^{k}\left(p^{2,1}\right)+i} \text { then } \\
\sum_{i=1}^{k+1}\left(p^{1, i}+p^{2, i}\right) \\
\text { if } L H S^{1, k+1}+\sum_{i=1}^{p^{2, k}} T_{\mu_{3}}^{2, \sum_{i=1}^{k+1}\left(p^{1, i}\right)+\sum_{i=1}^{k}\left(p^{2, i}\right)+i}<t<L H S^{1, k+1}+ \\
\sum_{i=1}^{p^{2, k}}\left(T_{\mu_{3}}^{2, \sum_{i=1}^{k+1}\left(p^{1, i}\right)+\sum_{i=1}^{k}\left(p^{2, i}\right)+i}\right)+T_{\mu_{3}}^{1, \sum_{i=1}^{k+1}\left(p^{1, i}+p^{2, i}\right)+1}\left(=L H S^{2, k+1}\right) \\
\sum_{i=1}^{k+1}\left(p^{1, i}+p^{2, i}\right)+1 \\
\text { if } L H S^{2, k+1}<t<L H S^{2, k+1}+\sum_{i=1}^{2} T_{\mu_{3}}^{1, \sum_{i=1}^{k+1}\left(p^{1, i}+p^{2, i}\right)+i} \\
\cdots \quad \cdots
\end{array}\right.
$$




\section{APPENDIX B. TWO-PART TYPE SYSTEMS: ANALYTICAL DERIVATIONS150}

Case 3: $T_{\lambda}^{j, i}+T_{\mu_{1}}^{j, i}<T_{\mu_{2}}^{j, i-1}$ and $T_{\mu_{3}}^{j, i-2} \geq T_{\mu_{2}}^{j, i-1}$ hold for each part $i$ of type $j$.

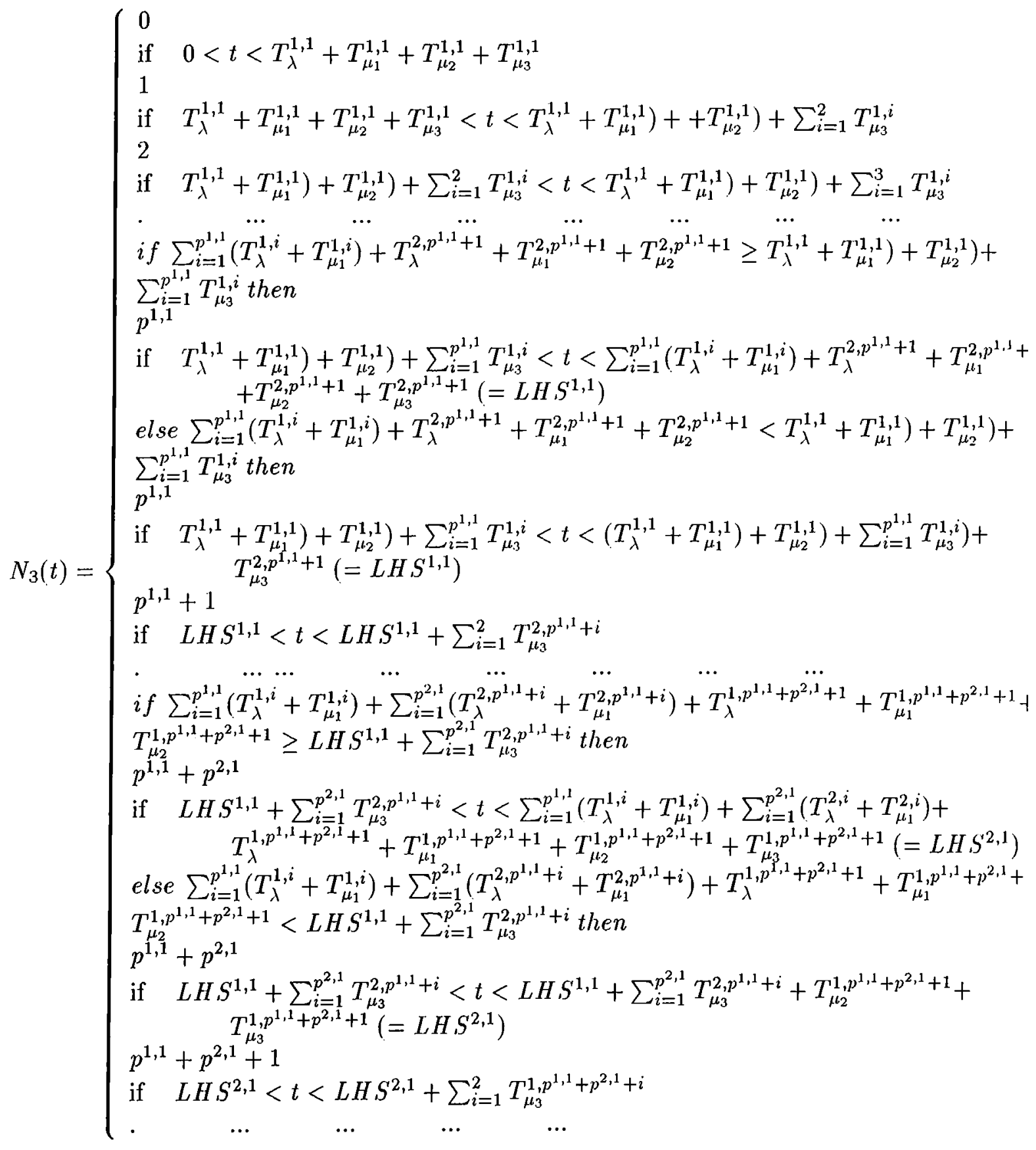




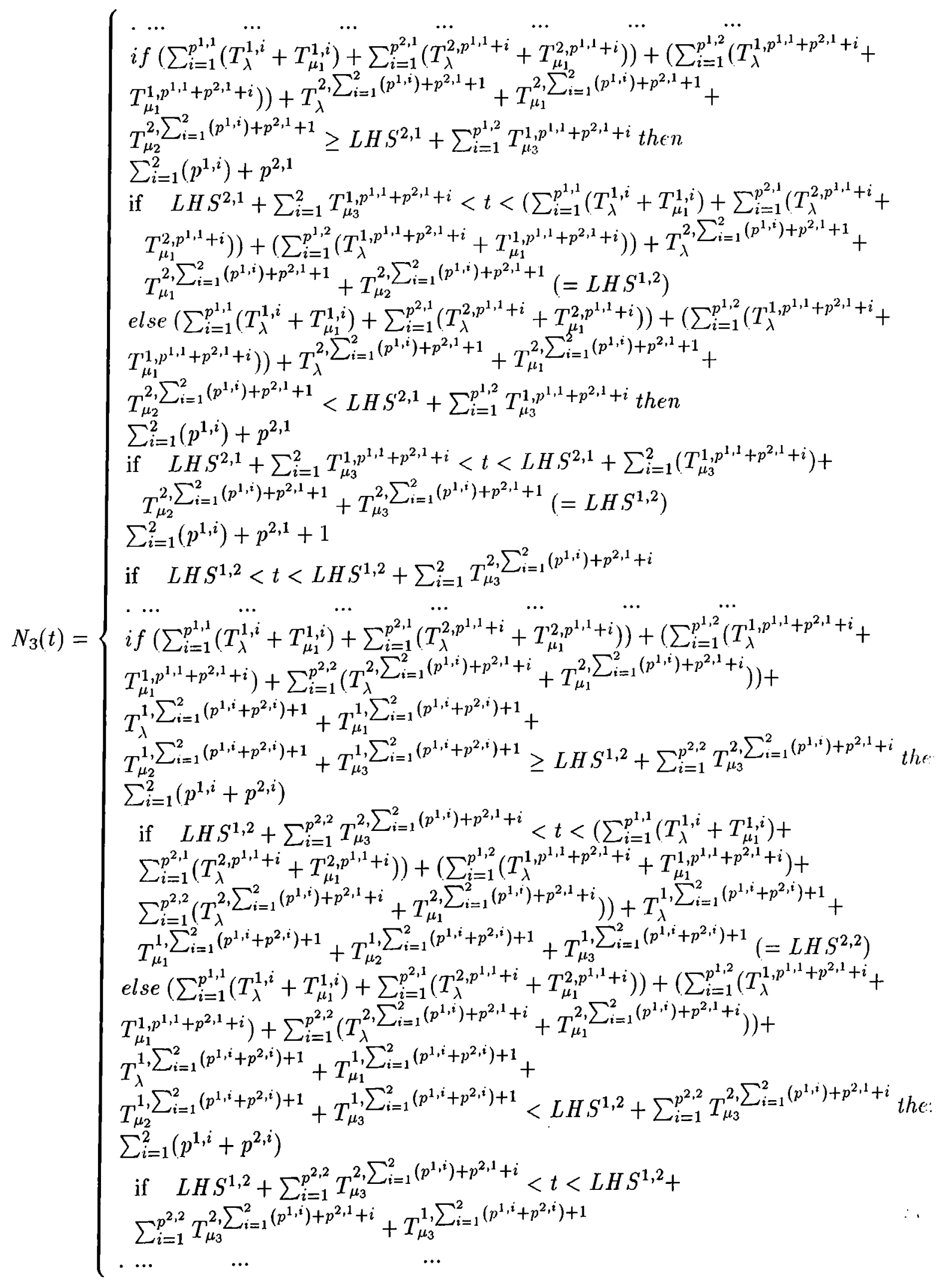




$$
\begin{aligned}
& \left\{\begin{array}{l}
\cdots \quad \cdots \quad \cdots \quad \cdots \quad \cdots \\
\text { if } \sum_{i=1}^{k}\left(\sum_{j=1}^{p^{1, i}}\left(T_{\lambda}^{1, \sum_{l=1}^{i-1}\left(p^{1, l}+p^{2, l}\right)+j}+T_{\mu_{1}}^{1, \sum_{l=1}^{i-1}\left(p^{1, l}+p^{2, l}\right)+j}\right)+\right. \\
\left.\sum_{j=1}^{p^{2, i}}\left(T_{\lambda}^{2, \sum_{l=1}^{i}\left(p^{1, l}\right)+\sum_{l=1}^{i-1}\left(p^{2, l}\right)+j}+T_{\mu_{1}}^{2, \sum_{l=1}^{i}\left(p^{1, l}\right)+\sum_{l=1}^{i-1}\left(p^{2, l}\right)+j}\right)\right)+ \\
T_{\lambda}^{1, \sum_{i=1}^{k}\left(p^{1, i}+p^{2, i}\right)+1}+T_{\mu_{1}}^{1, \sum_{i=1}^{k}\left(p^{1, i}+p^{2, i}\right)+1}+T_{\mu_{2}}^{1, \sum_{i=1}^{k}\left(p^{1, i}+p^{2, i}\right)+1}+ \\
T_{\mu_{3}}^{1, \sum_{i=1}^{k}\left(p^{1, i}+p^{2, i}\right)+1} \geq L H S^{1, k}+\sum_{i=1}^{p^{2, k-1}} T_{\mu_{3}}^{2, \sum_{i=1}^{k}\left(p^{1, i}\right)+\sum_{i=1}^{k-1}\left(p^{2,1}\right)+i} \text { then } \\
\sum_{i=1}^{k}\left(p^{1, i}+p^{2, i}\right)
\end{array}\right. \\
& \text { if } \quad L H S^{1, k}+\sum_{i=1}^{p^{2, k-1}} T_{\mu_{3}}^{2, \sum_{i=1}^{k}\left(p^{1, i}\right)+\sum_{i=1}^{k-1}\left(p^{2, i}\right)+i}<t< \\
& \sum_{i=1}^{k}\left(\sum_{j=1}^{p^{1, i}}\left(T_{\lambda}^{1, \sum_{l=1}^{i-1}\left(p^{1, l}+p^{2, l}\right)+j}+T_{\mu_{1}}^{1, \sum_{l=1}^{i-1}\left(p^{1, l}+p^{2, l}\right)+j}\right)+\right. \\
& \left.\sum_{j=1}^{p^{2, i}}\left(T_{\lambda}^{2, \sum_{l=1}^{i}\left(p^{1, l}\right)+\sum_{l=1}^{i-1}\left(p^{2, l}\right)+j}+T_{\mu_{1}}^{2, \sum_{l=1}^{i}\left(p^{1, l}\right)+\sum_{l=1}^{i-1}\left(p^{2, l}\right)+j}\right)\right)+ \\
& T_{\lambda}^{1, \sum_{i=1}^{k}\left(p^{1, i}+p^{2, i}\right)+1}+T_{\mu_{1}}^{1, \sum_{i=1}^{k}\left(p^{1, i}+p^{2, i}\right)+1}+T_{\mu_{2}}^{1, \sum_{i=1}^{k}\left(p^{1, i}+p^{2, i}\right)+1}+ \\
& T_{\mu_{3}}^{1, \sum_{i=1}^{k}\left(p^{1, i}+p^{2, i}\right)+1}\left(=L H, S^{2, k}\right) \\
& \text { else } \sum_{i=1}^{k}\left(\sum_{j=1}^{p^{1, i}}\left(T_{\lambda}^{1, \sum_{l=1}^{i-1}\left(p^{1, l}+p^{2, l}\right)+j}+T_{\mu_{1}}^{1, \sum_{l=1}^{i-1}\left(p^{1, l}+p^{2, l}\right)+j}\right)+\right. \\
& \left.\sum_{j=1}^{p^{2, i}}\left(T_{\lambda}^{2, \sum_{l=1}^{i}\left(p^{1, l}\right)+\sum_{l=1}^{i-1}\left(p^{2, l}\right)+j}+T_{\mu_{1}}^{2, \sum_{l=1}^{i}\left(p^{1, l}\right)+\sum_{l=1}^{i-1}\left(p^{2, l}\right)+j}\right)\right)+ \\
& T_{\lambda}^{1, \sum_{i=1}^{k}\left(p^{1, i}+p^{2, i}\right)+1}+T_{\mu_{1}}^{1, \sum_{i=1}^{k}\left(p^{1, i}+p^{2, i}\right)+1}+T_{\mu_{2}}^{1, \sum_{i=1}^{k}\left(p^{1, i}+p^{2, i}\right)+1}+ \\
& T_{\mu_{3}}^{1, \sum_{i=1}^{k}\left(p^{1, i}+p^{2, i}\right)+1}<L H S^{1, k}+\sum_{i=1}^{p^{2, k-1}} T_{\mu_{3}}^{2, \sum_{i=1}^{k}\left(p^{1, i}\right)+\sum_{i=1}^{k-1}\left(p^{2,1}\right)+i} \text { then } \\
& N_{3}(t)=\left\{\begin{array}{l}
T_{\mu_{3}}^{1, \sum_{i=1}}\left(p^{1, i}+p^{2, i}\right)+1 \\
\sum_{i=1}^{k}\left(p^{1, i}+p^{2, i}\right)
\end{array}\right. \\
& \text { if } \quad L H S^{1, k}+\sum_{i=1}^{p^{2, k-1}} T_{\mu_{3}}^{2, \sum_{i=1}^{k}\left(p^{1, i}\right)+\sum_{i=1}^{k-1}\left(p^{2, i}\right)+i}<t<L H S^{1, k}+ \\
& \sum_{i=1}^{p^{2, k-1}}\left(T_{\mu_{3}}^{2, \sum_{i=1}^{k}\left(p^{1, i}\right)+\sum_{i=1}^{k-1}\left(p^{2, i}\right)+i}\right)+T_{\mu_{3}}^{1, \sum_{i=1}^{k}\left(p^{1, i}+p^{2, i}\right)+1}\left(=L H S^{2, k}\right) \\
& \sum_{i=1}^{k}\left(p^{1, i}+p^{2, i}\right)+1 \\
& \text { if } \quad L H S^{2, k}<t<L H S^{2, k}+\sum_{i=1}^{2} T_{\mu_{3}}^{1, \sum_{i=1}^{k}\left(p^{1, i}+p^{2, i}\right)+i} \\
& \text { if } \sum_{i=1}^{k}\left(\sum _ { j = 1 } ^ { p ^ { 1 , i } } \left(T_{\lambda}^{1, \sum_{l=1}^{i-1}\left(p^{1, l}+p^{2, l}\right)+j}+\cdots \cdots \cdots\right.\right. \\
& \left.\sum_{j=1}^{p^{2, i}}\left(T_{\lambda}^{2, \sum_{l=1}^{l}\left(p^{1, l}\right)+\sum_{l=1}^{i-1}\left(p^{2, l}\right)+j}+T_{\mu_{1}}^{2, \sum_{l=1}^{i}\left(p^{1, l}\right)+\sum_{l=1}^{i-1}\left(p^{2, l}\right)+j}\right)\right)+ \\
& \left(\sum_{i=1}^{p^{1, k+1}}\left(T_{\lambda}^{1, \sum_{i=1}^{k}\left(p^{1, i}+p^{2, i}\right)+i}+T_{\mu_{1}}^{1, \sum_{i=1}^{k}\left(p^{1, i}+p^{2, i}\right)+i}\right)\right)+ \\
& T_{\lambda}^{2, \sum_{i=1}^{k+1}\left(p^{1, i}\right)+\sum_{i=1}^{k}\left(p^{2, i}\right)+1}+T_{\mu_{1}}^{2, \sum_{i=1}^{k+1}\left(p^{1, i}\right)+\sum_{i=1}^{k}\left(p^{2, i}\right)+1}+T_{\mu_{2}}^{2, \sum_{i=1}^{k+1}\left(p^{1, i}\right)+\sum_{i=1}^{k}\left(p^{2, i}\right)+1} \\
& \geq \text { LH S } S^{2, k}+\sum_{i=1}^{2} T_{\mu_{3}}^{1, \sum_{i=1}^{k}\left(p^{1, i}+p^{2, i}\right)+i} \text { then } \\
& \sum_{i=1}^{k+1}\left(p^{1, i}\right)+\sum_{i=1}^{k}\left(p^{2, i}\right) \\
& \text { if } \quad L H S^{2, k}+\sum_{i=1}^{p^{1, k+1}} T_{\mu_{3}}^{1, \sum_{i=1}^{k}\left(p^{1, i}+p^{2, i}\right)+i}<t< \\
& \sum_{i=1}^{k}\left(\sum_{j=1}^{p^{1, i}}\left(T_{\lambda}^{1, \sum_{l=1}^{i-1}\left(p^{1, l}+p^{2, l}\right)+j}+T_{\mu_{1}}^{1, \sum_{l=1}^{i-1}\left(p^{1, l}+p^{2, l}\right)+j}\right)+\right. \\
& \left.\sum_{j=1}^{p^{2, i}}\left(T_{\lambda}^{2, \sum_{l=1}^{i}\left(p^{1, l}\right)+\sum_{l=1}^{i-1}\left(p^{2, l}\right)+j}+T_{\mu_{1}}^{2, \sum_{l=1}^{i}\left(p^{1, l}\right)+\sum_{l=1}^{i-1}\left(p^{2, l}\right)+j}\right)\right)+ \\
& \left(\sum_{i=1}^{p^{1, k+1}}\left(T_{\lambda}^{1, \sum_{i=1}^{k}\left(p^{1, i}+p^{2, i}\right)+i}+T_{\mu_{1}}^{1, \sum_{i=1}^{k}\left(p^{1, i}+p^{2, i}\right)+i}\right)\right)+
\end{aligned}
$$




$$
\begin{aligned}
& \left\{\begin{array}{l}
T_{\lambda}^{2, \sum_{i=1}^{k+1}\left(p^{1, i}\right)+\sum_{i=1}^{k}\left(p^{2, i}\right)+1}+T_{\mu_{1}}^{2, \sum_{i=1}^{k+1}\left(p^{1, i}\right)+\sum_{i=1}^{k}\left(p^{2, i}\right)+1}+ \\
T_{\mu_{2}}^{2, \sum_{i=1}^{k+1}\left(p^{1, i}\right)+\sum_{i=1}^{k}\left(p^{2, i}\right)+1}\left(=L H S^{1, k+1}\right) \\
\operatorname{else} \sum_{i=1}^{k}\left(\sum_{j=1}^{p^{1, i}}\left(T_{\lambda}^{1, \sum_{l=1}^{i-1}\left(p^{1, l}+p^{2, l}\right)+j}+T_{\mu_{1}}^{1, \sum_{l=1}^{i-1}\left(p^{1, l}+p^{2, l}\right)+j}\right)+\right. \\
\left.\sum_{j=1}^{p^{2, i}}\left(T_{\lambda}^{2, \sum_{l=1}^{i}\left(p^{1, l}\right)+\sum_{l=1}^{i-1}\left(p^{2, l}\right)+j}+T_{\mu_{1}}^{2, \sum_{l=1}^{i}\left(p^{1, l}\right)+\sum_{l=1}^{i-1}\left(p^{2, l}\right)+j}\right)\right)+ \\
\left(\sum_{i=1}^{p^{1, k+1}}\left(T_{\lambda}^{1, \sum_{i=1}^{k}\left(p^{1, i}+p^{2, i}\right)+i}+T_{\mu_{1}}^{1, \sum_{i=1}^{k}\left(p^{1, i}+p^{2, i}\right)+i}\right)\right)+ \\
T_{\lambda}^{2, \sum_{i=1}^{k+1}\left(p^{1, i}\right)+\sum_{i=1}^{k}\left(p^{2, i}\right)+1}+T_{\mu_{1}}^{2, \sum_{i=1}^{k+1}\left(p^{1, i}\right)+\sum_{i=1}^{k}\left(p^{2, i}\right)+1}+T_{\mu_{2}}^{2, \sum_{i=1}^{k+1}\left(p^{1, i}\right)+\sum_{i=1}^{k}\left(p^{2, i}\right)+1} \\
<L H S^{2, k}+\sum_{i=1}^{2} T_{\mu_{3}}^{1, \sum_{i=1}^{k}\left(p^{1, i}+p^{2, i}\right)+i} \text { then } \\
\sum_{i=1}^{k+1}\left(p^{1, i}\right)+\sum_{i=1}^{k}\left(p^{2, i}\right)
\end{array}\right. \\
& \text { if } \quad L H S^{2, k}+\sum_{i=1}^{p^{1, k+1}} T_{\mu_{3}}^{1, \sum_{i=1}^{k}\left(p^{1, i}+p^{2, i}\right)+i}<t<L H S^{2, k}+ \\
& \sum_{i=1}^{p^{1, k+1}}\left(T_{\mu_{3}}^{1, \sum_{i=1}^{k}\left(p^{1, i}+p^{2, i}\right)+i}\right)+T_{\mu_{3}}^{2, \sum_{i=1}^{k+1}\left(p^{1, i}\right)+\sum_{i=1}^{k}\left(p^{2, i}\right)+1}\left(=L H S^{1, k+1}\right) \\
& \sum_{i=1}^{k+1}\left(p^{1, i}\right)+\sum_{i=1}^{k}\left(p^{2, i}\right)+1 \\
& \text { if } L H S^{1, k+1}<t<L H S^{1, k+1}+\sum_{i=1}^{2} T_{\mu_{3}}^{2, \sum_{i=1}^{k+1}\left(p^{1, i}\right)+\sum_{i=1}^{k}\left(p^{2, i}\right)+i} \\
& \text { if } \sum_{i=1}^{k+1}\left(\sum_{j=1}^{p^{1, i}}\left(T_{\lambda}^{1, \sum_{l=1}^{i-1}\left(p^{1, l}+p^{2, l}\right)+j}+T_{\mu_{1}}^{1, \sum_{l=1}^{i-1}\left(p^{1, l}+p^{2, l}\right)+j}\right)+\right. \\
& \left.\sum_{j=1}^{p^{2, i}}\left(T_{\lambda}^{2, \sum_{l=1}^{i}\left(p^{1, l}\right)+\sum_{l=1}^{i-1}\left(p^{2, l}\right)+j}+T_{\mu_{1}}^{2, \sum_{l=1}^{i}\left(p^{1, l}\right)+\sum_{l=1}^{i-1}\left(p^{2, l}\right)+j}\right)\right)+ \\
& T_{\lambda}^{1, \sum_{i=1}^{k+1}\left(p^{1, i}+p^{2, i}\right)+1}+T_{\mu_{1}}^{1, \sum_{i=1}^{k+1}\left(p^{1, i}+p^{2, i}\right)+1}+T_{\mu_{2}}^{1, \sum_{i=1}^{k+1}\left(p^{1, i}+p^{2, i}\right)+1}+
\end{aligned}
$$

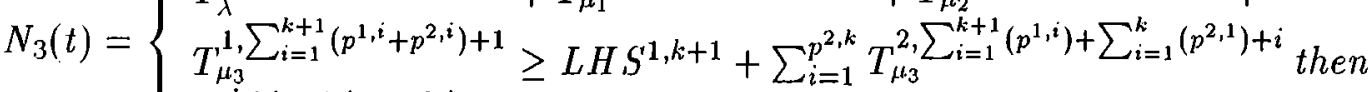

$$
\begin{aligned}
& \sum_{i=1}^{k+1}\left(p^{1, i}+p^{2, i}\right) \\
& \text { if } \quad L H S^{1, k+1}+\sum_{i=1}^{p^{2, k}} T_{\mu_{3}}^{2, \sum_{i=1}^{k+1}\left(p^{1, i}\right)+\sum_{i=1}^{k}\left(p^{2, i}\right)+i}<t< \\
& \sum_{i=1}^{k+1}\left(\sum_{j=1}^{p^{1, i}}\left(T_{\lambda}^{1, \sum_{l=1}^{i-1}\left(p^{1, l}+p^{2, l}\right)+j}+T_{\mu_{1}}^{1, \sum_{l=1}^{i-1}\left(p^{1, l}+p^{2, l}\right)+j}\right)+\right. \\
& \left.\sum_{j=1}^{p^{2, i}}\left(T_{\lambda}^{2, \sum_{l=1}^{i}\left(p^{1, l}\right)+\sum_{l=1}^{i-1}\left(p^{2, l}\right)+j}+T_{\mu_{1}}^{2, \sum_{l=1}^{i}\left(p^{1, l}\right)+\sum_{l=1}^{i-1}\left(p^{2, l}\right)+j}\right)\right)+ \\
& T_{\lambda}^{1, \sum_{i=1}^{k+1}\left(p^{1, i}+p^{2, i}\right)+1}+T_{\mu_{1}}^{1, \sum_{i=1}^{k+1}\left(p^{1, i}+p^{2, i}\right)+1}+T_{\mu_{2}}^{1, \sum_{i=1}^{k+1}\left(p^{1, i}+p^{2, i}\right)+1}+ \\
& T_{\mu_{3}}^{1, \sum_{i=1}^{k+1}\left(p^{1, i}+p^{2, i}\right)+1}\left(=L H S^{2, k+1}\right) \\
& \text { else } \sum_{i=1}^{k+1}\left(\sum_{j=1}^{p^{1, i}}\left(T_{\lambda}^{1, \sum_{l=1}^{i-1}\left(p^{1, l}+p^{2, l}\right)+j}+T_{\mu, 1}^{1, \sum_{l=1}^{i-1}\left(p^{1, l}+p^{2, l}\right)+j}\right)+\right. \\
& \left.\sum_{j=1}^{p^{2, i}}\left(T_{\lambda}^{2, \sum_{l=1}^{i}\left(p^{1, l}\right)+\sum_{l=1}^{i-1}\left(p^{2, l}\right)+j}+T_{\mu_{1}}^{2, \sum_{l=1}^{i}\left(p^{1, l}\right)+\sum_{l=1}^{i-1}\left(p^{2, l}\right)+j}\right)\right)+ \\
& T_{\lambda}^{1, \sum_{i=1}^{k+1}\left(p^{1, i}+p^{2, i}\right)+1}+T_{\mu_{1}}^{1, \sum_{i=1}^{k+1}\left(p^{1, i}+p^{2, i}\right)+1}+T_{\mu_{2}}^{1, \sum_{i=1}^{k+1}\left(p^{1, i}+p^{2, i}\right)+1}+ \\
& T_{\mu_{3}}^{1, \sum_{i=1}^{k+1}\left(p^{1, i}+p^{2, i}\right)+1}<L H S^{1, k+1}+\sum_{i=1}^{p^{2, k}} T_{\mu_{3}}^{2, \sum_{i=1}^{k+1}\left(p^{1, i}\right)+\sum_{i=1}^{k}\left(p^{2,1}\right)+i} \text { then } \\
& \sum_{i=1}^{k+1}\left(p^{1, i}+p^{2, i}\right) \\
& \text { if } L H S^{1, k+1}+\sum_{i=1}^{p^{2, k}} T_{\mu_{3}}^{2, \sum_{i=1}^{k+1}\left(p^{1, i}\right)+\sum_{i=1}^{k}\left(p^{2, i}\right)+i}<t<L H S^{1, k+1}+ \\
& \sum_{i=1}^{p^{2, k}}\left(T_{\mu_{3}}^{2, \sum_{i=1}^{k+1}\left(p^{1, i}\right)+\sum_{i=1}^{k}\left(p^{2, i}\right)+i}\right)+T_{\mu_{3}}^{1, \sum_{i=1}^{k+1}\left(p^{1, i}+p^{2, i}\right)+1}\left(=\text { LH } S^{2, k+1}\right) \\
& \sum_{i=1}^{k+1}\left(p^{1, i}+p^{2, i}\right)+1 \\
& \text { if } \quad L H S^{2, k+1}<t<L H S^{2, k+1}+\sum_{i=1}^{2} T_{\mu_{3}}^{1, \sum_{i=1}^{k+1}\left(p^{1, i}+p^{2, i}\right)+i}
\end{aligned}
$$




\section{APPENDIX B. TWO-PART TYPE SYSTEMS: ANALYTICAL DERIVATIONS154}

Case 4: $T_{\lambda}^{j, i}+T_{\mu_{1}}^{j, i}<T_{\mu_{2}}^{j, i-1}$ and $T_{\mu_{3}}^{j, i-2}<T_{\mu_{2}}^{j, i-1}$ hold for each part of type $j$.

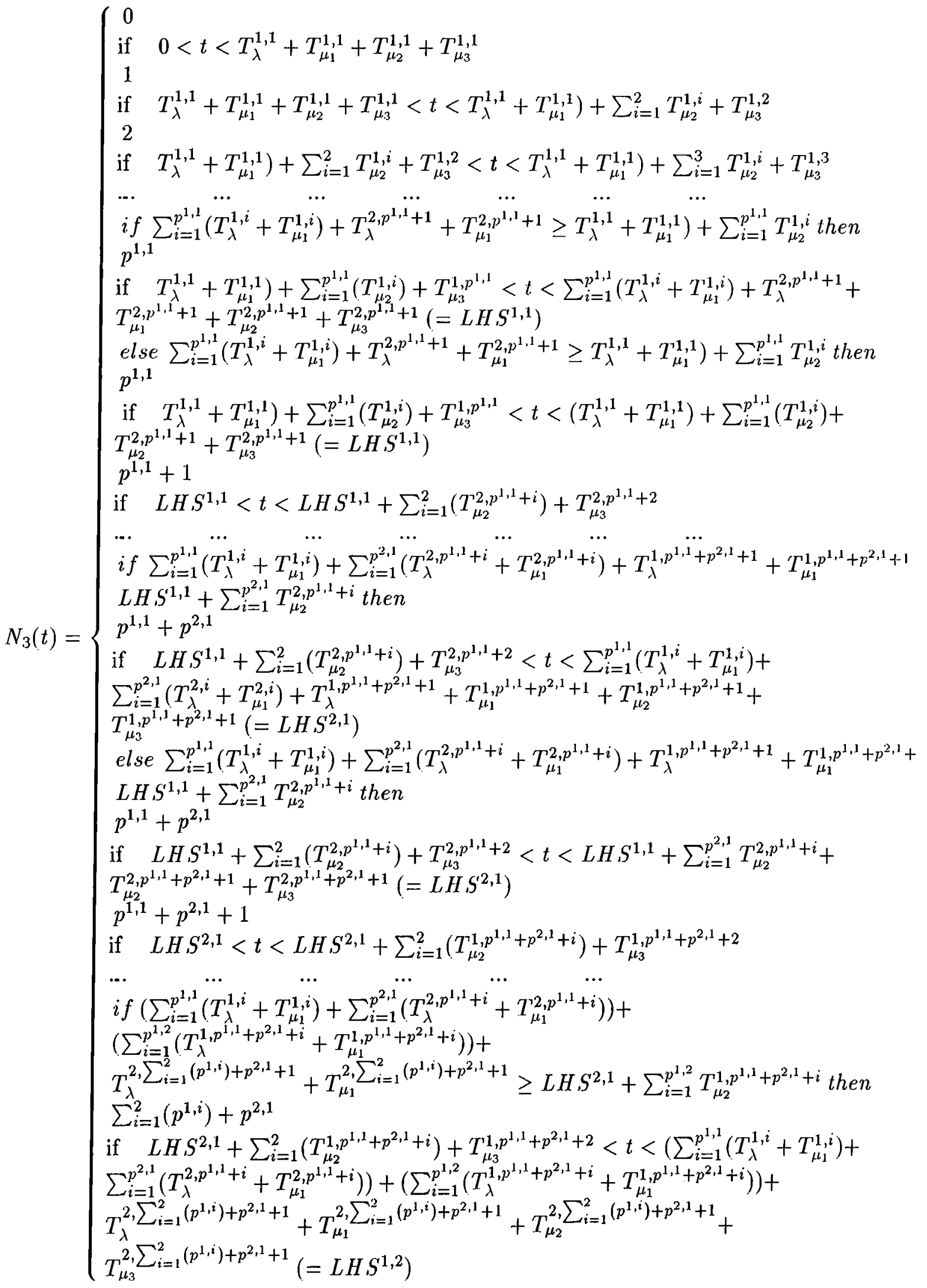




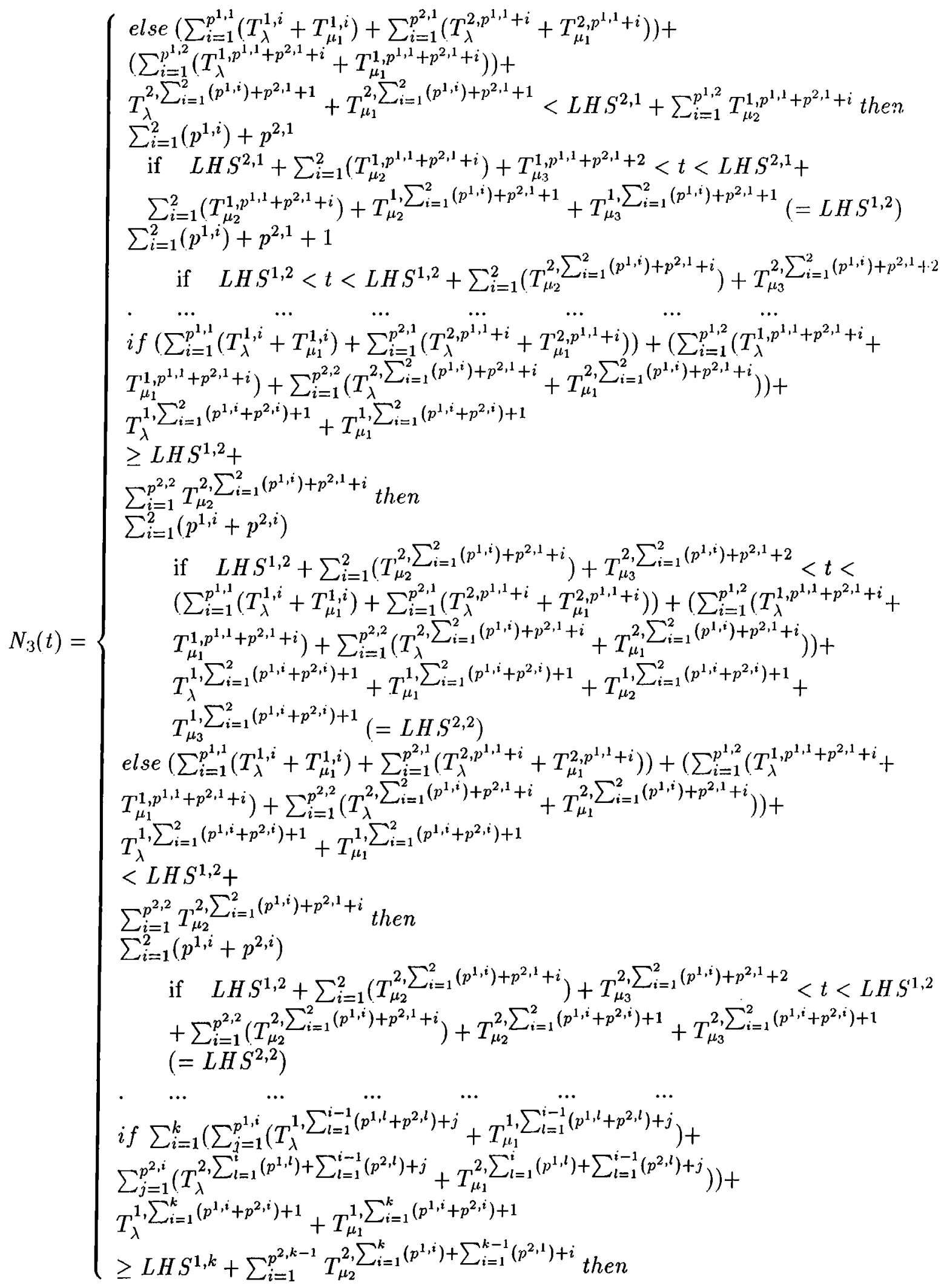




$$
\begin{aligned}
& \left\{\begin{array}{l}
\sum_{i=1}^{k}\left(p^{1, i}+p^{2, i}\right) \\
\text { if } L H S^{1, k}+\sum_{i=1}^{p^{2, k-1}}\left(T_{\mu_{2}}^{2, \sum_{i=1}^{k}\left(p^{1, i}\right)+\sum_{i=1}^{k-1}\left(p^{2, i}\right)+i}\right)+T_{\mu_{3}}^{2, \sum_{i=1}^{k}\left(p^{1, i}+p^{2, i}\right)}<t<
\end{array}\right. \\
& \sum_{i=1}^{k}\left(\sum_{j=1}^{p^{1, i}}\left(T_{\lambda}^{1, \sum_{l=1}^{i=1}\left(p^{1, l}+p^{2, l}\right)+j}+T_{\mu_{1}}^{1, \sum_{l=1}^{i-1}\left(p^{1, l}+p^{2, l}\right)+j}\right)+\right. \\
& \left.\sum_{j=1}^{p^{2, i}}\left(T_{\lambda}^{2, \sum_{l=1}^{i}\left(p^{1, l}\right)+\sum_{l=1}^{i-1}\left(p^{2, l}\right)+j}+T_{\mu_{1}}^{2, \sum_{l=1}^{i}\left(p^{1, l}\right)+\sum_{l=1}^{i-1}\left(p^{2, l}\right)+j}\right)\right)+ \\
& T_{\lambda}^{1, \sum_{i=1}^{k}\left(p^{1, i}+p^{2, i}\right)+1}+T_{\mu_{1}}^{1, \sum_{i=1}^{k}\left(p^{1, i}+p^{2, i}\right)+1}+T_{\mu_{2}}^{1, \sum_{i=1}^{k}\left(p^{1, i}+p^{2, i}\right)+1}+ \\
& T_{\mu 3}^{1, \sum_{i=1}^{k}\left(p^{1, i}+p^{2, i}\right)+1}\left(=\text { LH } S^{2, k}\right) \\
& \text { else } \sum_{i=1}^{k}\left(\sum_{j=1}^{p^{1, i}}\left(T_{\lambda}^{1, \sum_{l=1}^{i-1}\left(p^{1, l}+p^{2, l}\right)+j}+T_{\mu_{1}}^{1, \sum_{l=1}^{i-1}\left(p^{1, l}+p^{2, l}\right)+j}\right)+\right. \\
& \left.\sum_{j=1}^{p^{2, i}}\left(T_{\lambda}^{2, \sum_{l=1}^{i}\left(p^{1, l}\right)+\sum_{l=1}^{i-1}\left(p^{2, l}\right)+j}+T_{\mu_{1}}^{2, \sum_{l=1}^{i}\left(p^{1, l}\right)+\sum_{l=1}^{i-1}\left(p^{2, l}\right)+j}\right)\right)+ \\
& T_{\lambda}^{1, \sum_{i=1}^{k}\left(p^{1, i}+p^{2, i}\right)+1}+T_{\mu_{1}}^{1, \sum_{i=1}^{k}\left(p^{1, i}+p^{2, i}\right)+1} \\
& <L H S^{1, k}+\sum_{i=1}^{p^{2, k-1}} T_{\mu_{2}}^{2, \sum_{i=1}^{k}\left(p^{1, i}\right)+\sum_{i=1}^{k-1}\left(p^{2,1}\right)+i} \text { then } \\
& \sum_{i=1}^{k}\left(p^{1, i}+p^{2, i}\right) \\
& \text { if } L H S^{1, k}+\sum_{i=1}^{p^{2, k-1}}\left(T_{\mu_{2}}^{2, \sum_{i=1}^{k}\left(p^{1, i}\right)+\sum_{i=1}^{k-1}\left(p^{2, i}\right)+i}\right)+T_{\mu_{3}}^{2, \sum_{i=1}^{k}\left(p^{1, i}+p^{2, i}\right)} \\
& <t<L H S^{1, k}+\sum_{i=1}^{p^{2, k-1}}\left(T_{\mu_{2}}^{2, \sum_{i=1}^{k}\left(p^{1, i}\right)+\sum_{i=1}^{k-1}\left(p^{2, i}\right)+i}\right)+T_{\mu_{2}}^{1, \sum_{i=1}^{k}\left(p^{1, i}+p^{2, i}\right)+1} \\
& +T_{\mu_{3}}^{1, \sum_{i=1}^{k}\left(p^{1, i}+p^{2, i}\right)+1}\left(=L H S^{2, k}\right) \\
& N_{3}(t)=\left\{\begin{array}{l}
\sum_{i=1}^{k}\left(p^{1, i}+p^{2, i}\right)+1 \\
\text { if } L H S^{2, k}<t<L H S^{2, k}+\sum_{i=1}^{2}\left(T_{\mu_{2}}^{1, \sum_{i=1}^{k}\left(p^{1, i}+p^{2, i}\right)+i}\right)+
\end{array}\right. \\
& T_{\mu_{3}}^{1, \sum_{i=1}^{k}\left(p^{1, i}+p^{2, i}\right)+2} \\
& \text { if } \sum_{i=1}^{k}\left(\sum_{j=1}^{p^{1, i}}\left(T_{\lambda}^{1, \sum_{l=1}^{i-1}\left(p^{1, l}+p^{2, l}\right)+j}+T_{\mu_{1}}^{1, \sum_{l=1}^{i-1}\left(p^{1, l}+p^{2, l}\right)+j}\right)+\right. \\
& \left.\sum_{j=1}^{p^{2, i}}\left(T_{\lambda}^{2, \sum_{l=1}^{i}\left(p^{1, l}\right)+\sum_{l=1}^{i-1}\left(p^{2, l}\right)+j}+T_{\mu_{1}}^{2, \sum_{l=1}^{i}\left(p^{1, l}\right)+\sum_{l=1}^{i-1}\left(p^{2, l}\right)+j}\right)\right)+ \\
& \left(\sum_{i=1}^{p^{1, k+1}}\left(T_{\lambda}^{1, \sum_{i=1}^{k}\left(p^{1, i}+p^{2, i}\right)+i}+T_{\mu_{1}}^{1, \sum_{i=1}^{k}\left(p^{1, i}+p^{2, i}\right)+i}\right)\right)+ \\
& T_{\lambda}^{2, \sum_{i=1}^{k+1}\left(p^{1, i}\right)+\sum_{i=1}^{k}\left(p^{2, i}\right)+1}+T_{\mu_{1}}^{2, \sum_{i=1}^{k+1}\left(p^{1, i}\right)+\sum_{i=1}^{k}\left(p^{2, i}\right)+1} \\
& \geq L H S^{2, k}+\sum_{i=1}^{p^{1, k+1}} T_{\mu_{2}}^{1, \sum_{i=1}^{k}\left(p^{1, i}+p^{2, i}\right)+i} \text { then } \\
& \sum_{i=1}^{k+1}\left(p^{1, i}\right)+\sum_{i=1}^{k}\left(p^{2, i}\right) \\
& \text { if } \quad L H S^{2, k}+\sum_{i=1}^{p^{1, k+1}}\left(T_{\mu_{2}}^{1, \sum_{i=1}^{k}\left(p^{1, i}+p^{2, i}\right)+i}\right)+T_{\mu_{3}}^{1, \sum_{i=1}^{k+1}\left(p^{1, i}\right)+\sum_{i=1}^{k}\left(p^{2, i}\right)} \\
& <t<\sum_{i=1}^{k}\left(\sum_{j=1}^{p^{1, i}}\left(T_{\lambda}^{1, \sum_{l=1}^{i-1}\left(p^{1, l}+p^{2, l}\right)+j}+T_{\mu_{1}}^{1, \sum_{l=1}^{i-1}\left(p^{1, l}+p^{2, l}\right)+j}\right)+\right. \\
& \left.\sum_{j=1}^{p^{2, i}}\left(T_{\lambda}^{2, \sum_{l=1}^{i}\left(p^{1, l}\right)+\sum_{l=1}^{i-1}\left(p^{2, l}\right)+j}+T_{\mu_{1}}^{2, \sum_{l=1}^{i}\left(p^{1, l}\right)+\sum_{l=1}^{i-1}\left(p^{2, l}\right)+j}\right)\right)+ \\
& \left(\sum_{i=1}^{p^{1, k+1}}\left(T_{\lambda}^{1, \sum_{l=1}^{i-1}\left(p^{1, l}+p^{2, l}\right)+i}+T_{\mu_{1}}^{1, \sum_{l=1}^{k}\left(p^{1, l}+p^{2, l}\right)+i}\right)\right)+ \\
& T_{\lambda}^{2, \sum_{i=1}^{k+1}\left(p^{1, i}\right)+\sum_{i=1}^{k}\left(p^{2, i}\right)+1}+T_{\mu_{1}}^{2, \sum_{i=1}^{k+1}\left(p^{1, i}\right)+\sum_{i=1}^{k}\left(p^{2, i}\right)+1}+ \\
& T_{\mu_{2}}^{2, \sum_{i=1}^{k+1}\left(p^{1, i}\right)+\sum_{i=1}^{k}\left(p^{2, i}\right)+1}+T_{\mu_{3}}^{2, \sum_{i=1}^{k+1}\left(p^{1, i}\right)+\sum_{i=1}^{k}\left(p^{2, i}\right)+1}\left(=L H S^{1, k+1}\right)
\end{aligned}
$$




$$
\begin{aligned}
& \left\{\begin{array}{l}
\text { else } \sum_{i=1}^{k}\left(\sum_{j=1}^{p^{1, i}}\left(T_{\lambda}^{1, \sum_{l=1}^{i-1}\left(p^{1, l}+p^{2, l}\right)+j}+T_{\mu_{1}}^{1, \sum_{l=1}^{i-1}\left(p^{1, l}+p^{2, l}\right)+j}\right)+\right. \\
\left.\sum_{j=1}^{p^{2, i}}\left(T_{\lambda}^{2, \sum_{l=1}^{i}\left(p^{1, i}\right)+\sum_{l=1}^{i-1}\left(p^{2, l}\right)+j}+T_{\mu_{1}}^{2, \sum_{l=1}^{i}\left(p^{1, l}\right)+\sum_{l=1}^{i-1}\left(p^{2, l}\right)+j}\right)\right)+ \\
\left(\sum_{i=1}^{p^{1, k+1}}\left(T_{\lambda}^{1, \sum_{i=1}^{k}\left(p^{1, i}+p^{2, i}\right)+i}+T_{\mu_{1}}^{1, \sum_{i=1}^{k}\left(p^{1, i}+p^{2, i}\right)+i}\right)\right)+ \\
T_{\lambda}^{2, \sum_{i=1}^{k+1}\left(p^{1, i}\right)+\sum_{i=1}^{k}\left(p^{2, i}\right)+1}+T_{\mu_{1}}^{2, \sum_{i=1}^{k+1}\left(p^{1, i}\right)+\sum_{i=1}^{k}\left(p^{2, i}\right)+1} \\
<L H S^{2, k}+\sum_{i=1}^{p^{1, k+1}} T_{\mu_{2}}^{1, \sum_{i=1}^{k}\left(p^{1, i}+p^{2, i}\right)+i} \text { then } \\
\sum_{i=1}^{k+1}\left(p^{1, i}\right)+\sum_{i=1}^{k}\left(p^{2, i}\right) \\
\text { if } \quad L H S^{2, k}+\sum_{i=1}^{p^{1, k+1}}\left(T_{\mu_{2}}^{1, \sum_{i=1}^{k}\left(p^{1, i}+p^{2, i}\right)+i}\right)+T_{\mu_{3}}^{1, \sum_{i=1}^{k+1}\left(p^{1, i}\right)+\sum_{i=1}^{k}\left(p^{2, i}\right)} \\
<t<L H S^{2, k}+\sum_{i=1}^{p^{1, k+1}}\left(T_{\mu_{2}}^{1, \sum_{i=1}^{k}\left(p^{1, i}+p^{2, i}\right)+i}\right)+T_{\mu_{2}}^{1, \sum_{i=1}^{k+1}\left(p^{1, i}\right)+\sum_{i=1}^{k}\left(p^{2, i}\right)+1} \\
+T_{\mu_{3}}^{1, \sum_{i=1}^{k+1}\left(p^{1, i}\right)+\sum_{i=1}^{k}\left(p^{2, i}\right)+1}\left(=L H S^{1, k+1}\right) \\
\sum_{i=1}^{k+1}\left(p^{1, i}\right)+\sum_{i=1}^{k}\left(p^{2, i}\right)+1
\end{array}\right. \\
& \text { if } L H S^{1, k+1}<t<L H, S^{1, k+1}+\sum_{i=1}^{2}\left(T_{\mu_{2}}^{2, \sum_{i=1}^{k+1}\left(p^{1, i}\right)+\sum_{i=1}^{k}\left(p^{2, i}\right)+i}\right)+ \\
& T_{\mu_{3}}^{2, \sum_{i=1}^{k+1}\left(p^{1, i}\right)+\sum_{i=1}^{k}\left(p^{2, i}\right)+2} \\
& \text { if } \sum_{i=1}^{k+1}\left(\sum_{j=1}^{p^{1, i}}\left(T_{\lambda}^{1, \sum_{l=1}^{i-1}\left(p^{1, l}+p^{2, l}\right)+j}+T_{\mu_{1}}^{1, \sum_{l=1}^{i-1}\left(p^{1, l}+p^{2, l}\right)+j}\right)+\right. \\
& \sum_{j=1}^{p^{2, i}}\left(T_{\lambda}^{2, \sum_{l=1}^{i}\left(p^{1, l}\right)+\sum_{l=1}^{i-1}\left(p^{2, l}\right)+j}+T_{\mu_{1}}^{2, \sum_{l=1}^{i}\left(p^{1, l}\right)+}\right. \\
& \left.\left.\sum_{l=1}^{i-1}\left(p^{2, l}\right)+j\right)\right)+T_{\lambda}^{1, \sum_{i=1}^{k+1}\left(p^{1, i}+p^{2, i}\right)+1}+T_{\mu_{1}}^{1, \sum_{i=1}^{k+1}\left(p^{1, i}+p^{2, i}\right)+1}+ \\
& \geq L H S^{1, k+1}+\sum_{i=1}^{p^{2, k}} T_{\mu_{2}}^{2, \sum_{i=1}^{k+1}\left(p^{1, i}\right)+\sum_{i=1}^{k}\left(p^{2,1}\right)+i} \text { then } \\
& N_{3}(t)=\left\{\begin{array}{l}
\geq L H S^{1, k+1}+\sum_{i=1}^{p^{2}} T_{\mu_{2}} \text { then } \\
\sum_{i=1}^{k+1}\left(p^{1, i}+p^{2, i}\right) \\
\text { if } L H S^{1, k+1}+\sum_{i=1}^{p^{2, k}}\left(T_{\mu_{2}}^{2, \sum_{i=1}^{k+1}\left(p^{1, i}\right)+\sum_{i=1}^{k}\left(p^{2, i}\right)+i}\right)+T_{\mu_{3}}^{2,} \\
\sum_{i=1}^{k+1}\left(\sum_{j=1}^{p^{1, i}}\left(T_{\lambda}^{1, \sum_{l=1}^{i-1}\left(p^{1, l}+p^{2, l}\right)+j}+T_{\mu_{1}}^{1, \sum_{l=1}^{i-1}\left(p^{1, l}+p^{2, l}\right)+j}\right)+\right.
\end{array}\right. \\
& \left.\sum_{j=1}^{p^{2, i}}\left(T_{\lambda}^{2, \sum_{l=1}^{i}\left(p^{1, l}\right)+\sum_{l=1}^{i-1}\left(p^{2, l}\right)+j}+T_{\mu_{1}}^{2, \sum_{l=1}^{i}\left(p^{1, l}\right)+\sum_{l=1}^{i-1}\left(p^{2, l}\right)+j}\right)\right)+ \\
& T_{\lambda}^{1, \sum_{i=1}^{k+1}\left(p^{1, i}+p^{2, i}\right)+1}+T_{\mu_{1}}^{1, \sum_{i=1}^{k+1}\left(p^{1, i}+p^{2, i}\right)+1}+T_{\mu_{2}}^{1, \sum_{i=1}^{k+1}\left(p^{1, i}+p^{2, i}\right)+1}+ \\
& T_{\mu_{3}}^{1, \sum_{i=1}^{k+1}\left(p^{1, i}+p^{2, i}\right)+1}\left(=L H S^{2, k+1}\right) \\
& \text { else } \sum_{i=1}^{k+1}\left(\sum_{j=1}^{p^{1, i}}\left(T_{\lambda}^{1, \sum_{l=1}^{i-1}\left(p^{1, l}+p^{2, l}\right)+j}+T_{\mu_{1}}^{1, \sum_{l=1}^{i-1}\left(p^{1, l}+p^{2, l}\right)+j}\right)+\right. \\
& \sum_{j=1}^{p^{2, i}}\left(T_{\lambda}^{2, \sum_{l=1}^{i}\left(p^{1, l}\right)+\sum_{l=1}^{i-1}\left(p^{2, l}\right)+j}+T_{\mu 1}^{2, \sum_{l=1}^{i}\left(p^{1, l}\right)+}\right. \\
& \left.\left.\sum_{l=1}^{i-1}\left(p^{2, l}\right)+j\right)\right)+T_{\lambda}^{1, \sum_{i=1}^{k+1}\left(p^{1, i}+p^{2, i}\right)+1}+T_{\mu_{1}}^{1, \sum_{i=1}^{k+1}\left(p^{1, i}+p^{2, i}\right)+1}+ \\
& <L H S^{1, k+1}+\sum_{i=1}^{p^{2, k}} T_{\mu_{2}}^{2, \sum_{i=1}^{k+1}\left(p^{1, i}\right)+\sum_{i=1}^{k}\left(p^{2,1}\right)+i} \text { then } \\
& \sum_{i=1}^{k+1}\left(p^{1, i}+p^{2, i}\right) \\
& \text { if } \quad L H S^{1, k+1}+\sum_{i=1}^{p^{2, k}}\left(T_{\mu_{2}}^{2, \sum_{i=1}^{k+1}\left(p^{1, i}\right)+\sum_{i=1}^{k}\left(p^{2, i}\right)+i}\right)+T_{\mu_{3}}^{2, \sum_{i=1}^{k+1}\left(p^{1, i}+p^{2, i}\right)} \\
& <t<L H S^{1, k+1}+\sum_{i=1}^{p^{2, k}}\left(T_{\mu_{2}}^{2, \sum_{i=1}^{k+1}\left(p^{1, i}\right)+\sum_{i=1}^{k}\left(p^{2, i}\right)+i}\right)+ \\
& T_{\mu_{2}}^{1, \sum_{i=1}^{k+1}\left(p^{1, i}+p^{2, i}\right)+1}+T_{\mu_{3}}^{1, \sum_{i=1}^{k+1}\left(p^{1, i}+p^{2, i}\right)+1}\left(=L H S^{2, k+1}\right) \\
& \sum_{i=1}^{k+1}\left(p^{1, i}+p^{2, i}\right)+1 \\
& \text { if } L H S^{2, k+1}<t<L H S^{2, k+1}+\sum_{i=1}^{2}\left(T_{\mu_{2}}^{1, \sum_{i=1}^{k+1}\left(p^{1, i}+p^{2, i}\right)+i}\right)+ \\
& T_{\mu_{3}}^{1, \sum_{i=1}^{k+1}\left(p^{1, i}+p^{2, i}\right)+2}
\end{aligned}
$$




\section{VITA}

Bahar Deler was born on March 21, 1974 in Zonguldak, Turkey. She received her high school education at Ali Osman Sönmez Science High School, Turkey. She has graduated from the Department of Industrial Engineering, Bilkent University, June 1996. In October 1996, she joined the Department of Industrial Engineering at Bilkent University. From that time to the present, she worked with Assoc. Prof. Cemal Dinçer for her graduate study at the same department. 